\title{
Use of commercial CFD software for assessing the performance of personal aerosol samplers
}

Aaron J. Bird

West Virginia University

Follow this and additional works at: https://researchrepository.wvu.edu/etd

\section{Recommended Citation}

Bird, Aaron J., "Use of commercial CFD software for assessing the performance of personal aerosol samplers" (2004). Graduate Theses, Dissertations, and Problem Reports. 2623.

https://researchrepository.wvu.edu/etd/2623

This Dissertation is protected by copyright and/or related rights. It has been brought to you by the The Research Repository @ WVU with permission from the rights-holder(s). You are free to use this Dissertation in any way that is permitted by the copyright and related rights legislation that applies to your use. For other uses you must obtain permission from the rights-holder(s) directly, unless additional rights are indicated by a Creative Commons license in the record and/ or on the work itself. This Dissertation has been accepted for inclusion in WVU Graduate Theses, Dissertations, and Problem Reports collection by an authorized administrator of The Research Repository @ WVU.

For more information, please contact researchrepository@mail.wvu.edu. 


\section{Use of Commercial CFD Software for Assessing the Performance of Personal Aerosol Samplers}

Aaron J. Bird

\section{Doctoral Dissertation}

Submitted to

The College of Engineering and Mineral Resources

West Virginia University

In partial fulfillment of the requirements for the degree of

Doctor of Philosophy

in

Industrial and Management Systems Engineering

Warren R. Myers, Ph.D., WVU-CEMR, Committee Chair

Ismail Celik, Ph.D., WVU-MAE, Benedum Scholar

Teh-hsun (Bean) Chen, Ph.D., CDC-NIOSH

Bhaskaran Gopalakrishnan, Ph.D., WVU-IMSE

Steven Guffey, Ph.D., WVU-IMSE

Wafik Iskander, Ph.D., WVU-IMSE

Department of Industrial and Management Systems Engineering

West Virginia University

in collaboration with

Centers for Disease Control and Prevention

National Institute for Occupational Safety and Health

Health Effects Laboratory Division

Morgantown, West Virginia

2004

Keywords: Aerosol Sampling; Computational Fluid Dynamics;

Industrial Hygiene; Personal Sampler

Copyright 2004 Aaron J. Bird 


\title{
Abstract \\ Use of Commercial CFD Software for Assessing the Performance of Personal Aerosol Samplers
}

\begin{abstract}
Aaron J. Bird
Accurate performance of personal aerosol samplers is critical for industrial hygienists' successful efforts to protect workers. Performance assessments of personal samplers are necessarily conducted in controlled environments so unknown sources of variability will not influence the results. This is done in order that samplers may be used with confidence for monitoring workplace air quality. These performance evaluations are usually conducted in wind tunnels. However, recent advances in high-performance cluster computing -together with advanced flow modeling software- have made it possible to evaluate sampler performance with computer simulations. This was done in the current research. Simulations were conducted for air and particle behavior near the inlet of the GSP and IOM Inhalable aerosol samplers using a pre-verified commercial computational fluid dynamics (CFD) software package. Steady-state, 3-D simulations were conducted using the FLUENT CFD solver with renormalized group (RNG) theory applied to a $k-\epsilon$ turbulence model. Particle trajectories were calculated in a Lagrangian reference frame on the resulting velocity fields. Based on the particle trajectories, sampling efficiencies were calculated and were compared to those reported in the literature. They were found to have similar overall trends for particle sizes up to $40 \mu \mathrm{m}$. Using a correction factor, agreement was observed to be reasonable for most cases. It was concluded that CFD can be used to evaluate the performance of personal samplers.
\end{abstract}




\section{Acknowledgements}

This dissertation and all that it has encompassed would have been impossible without the love and support of my wife, Rachel, my son, Tristan, and my daughter, Samantha. Their sacrifices have made this work possible and I dedicate it to them. I consider myself lucky for the support I have received from all of my family members and I would like to thank my sister, Sara; my brother, Ben; my mother, Karen; my stepmother, Donetta; my father, Fred; and my grandmother, Catherine, for their continued encouragement both academically and in life.

I am grateful for the support and guidance given to me by the many employees of NIOSH in Morgantown, WV. For so many things, I am thankful to Sid Soderholm, Bean Chen, Vladimir Hnizdo, Michael Andrew, Bill Wallace, Omur Elci, Greg Feather, Jennifer Hornsby-Myers, Mike Flemmer, Larry Lee, Norma McKee, Johnathan Barnett, Martin Harper, Donna Hucik, Joyce Counts, Janet Feather, and Crystal Forester. I would like to thank Jerry Smith at NIOSH in Cincinnati, OH for sharing his laser Doppler-velocimetry data with me and offering me the opportunity to help him analyze and publish the data. I will always be indebted to Paul Baron at NIOSH in Cincinnati, $\mathrm{OH}$ for his gracious guidance in the field of aerosol sampling and flow-field measurement. I am grateful to Al Munson and Nancy Bollinger at NIOSH in Morgantown for giving me the opportunity to do this stimulating research. I am forever indebted to the Administrative Team of the Exposure Assessment Branch, the Administrative Team of the Office of the Director for the Health Effects Laboratory Division, the personnel at the Lake Lynn Laboratory, and everyone else at NIOSH who have helped make this research effort a successful one. For my office mates at NIOSH, including Robert Konecny, Gennady Poda, Joshua Day, Adam Fedorowicz, Vladimir Murashov, and Travis Michette: I greatly value the good times we 
have shared and thank each of you for your friendship, insight, suggestions, and support, because, with it I have become a better scientist and a more complete person. Finally, I wish to thank everyone at NIOSH for being such wonderful and supportive people: the field of occupational safety and health is bettered every day through your work.

As with all research, this effort is based on the great accomplishments in the field of aerosol sampling by which it has been preceded. Many of the contributors to the field are acknowledged in the literature review section through what I hope are accurate and respectful descriptions of their research efforts. Of them, I specifically wish to thank the following two: first, Dr. Lee Kenny, Health and Safety Executive, London, England, for sharing results of the European study on aerosol samplers that she and her colleagues conducted, and second, Dr. Shou-Nan Li, Industrial Technology Research Institute, Taiwan, for sharing the results of his aerosol sampler Ph.D. work he did at the University of Florida.

In addition, I would like to specifically thank the friendly reviewers who carefully checked this document for typos, grammatical and punctuation mistakes, and figure/table agreement within the text. They are: Rachel Bosch, Omur Elci, Karen Bird, Sara Bird, Tad Kato, and the members of my dissertation committee. I also wish to acknowledge Mark Passerby for advice on graphics and associated software.

I would like to acknowledge the members of my dissertation committee in the WVU College of Engineering because with them, so much has become possible. I wish to thank Dr. Bhaskaran Gopala for his kind words of support throughout my graduate experience and his dedication to the quality education of Industrial Engineers. I would like to acknowledge Dr. Steven Guffey for his passion for research in the field of occupational safety and health and thank him for his ideas and suggestions. I am indebted to Dr. Wafik Iskander for sharing with me his wonderful qualities of patience, support, and kindness, and I would like to thank him for his insight in the field of statistics. I thank Dr. Ismail Celik for his 
patience and guidance as well as the challenges he has given me so that I could better know the subject of fluid dynamics. Last, I thank my advisor, Dr. Warren Myers, for bringing out potential in me that I myself did not know existed. 


\section{Symbols and Nomenclature}

$\begin{array}{ll}\mathrm{m} & \text { meters } \\ \mu \mathrm{m} & \text { micrometers } \\ \mathrm{s} & \text { seconds } \\ \mathrm{min} & \text { minutes } \\ \mathrm{g} & \text { gravity } \\ \mathrm{L} & \text { liters } \\ \mathrm{Q} & \text { flowrate } \\ \mathrm{V} & \text { velocity } \\ \mathrm{A} & \text { area } \\ y^{+} & \text {y-plus distance } \\ \Gamma_{e} & \text { scalar value, "East" side of grid } \\ \Gamma_{w} & \text { scalar value, "West" side of grid } \\ \delta_{x} & \text { distance between x-directional "East" and "West" grid nodes } \\ \delta_{y} & \text { distance between y-directional "North" and "South" grid nodes } \\ P & \text { "point" in center of grid being calculated } \\ \mathrm{N}, \mathrm{S}, \mathrm{E}, \mathrm{W} & \text { ordinal directions of grid } \\ S_{u} & \text { source term } \\ \partial & \text { partial derivative } \\ \rho & \text { density } \\ \phi & \text { scalar value } \\ \mathrm{d} & \text { particle aerodynamic diameter } \\ \mathrm{U}_{\mathrm{s}} & \text { sampler inlet velocity } \\ \mathrm{h} & 1 / 2 \text { inlet diameter } \\ \mu & \text { fluid viscosity } \\ & \end{array}$




\section{Abbreviations}

CFD computational fluid dynamics

DOE design of experiments

RE Reynolds number

ST Stokes number

MAE Mechanical and Aerospace Engineering

IMSE Industrial and Management Systems Engineering 


\section{Contents}

1 Introduction 1

2 Literature Review $\quad 23$

2.1 Chronology of Air Sampling Efficiency Studies . . . . . . . . . . . . 23

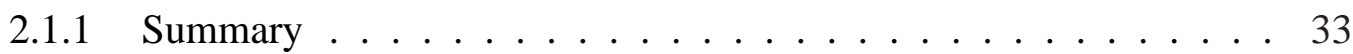

2.2 Development and Application of Theories in Aerosol Sampling . . . . . . . 33

2.2.1 Aerosol Aspiration in Human Models: Inhalability Convention . . . 34

2.2.2 Theoretical and Experimental Methods for Determining the Reference or "True" Concentration - The Stokes Number Approach . . . 37

2.2.3 Numerical Methods for Determining the Reference Concentration . 49

2.2 .4 Summary .......................... 52

2.3 Background for Use of CFD in Calculating Efficiency of Aerosol Samplers 53

2.3.1 Determination of Airflow Velocity . . . . . . . . . . . . 53

2.3.2 Calculation of Particle Trajectories in a Lagrangian Reference Frame 54

2.3 .3 Summary . . . . . . . . . . . . . . . . 59 
3 Research Methods $\quad 60$

3.1 Computational Fluid Dynamics . . . . . . . . . . . . . . . . . . 60

3.1.1 Governing Equations . . . . . . . . . . . . . . . . 60

3.1.2 Geometries for Simulations of Personal Aerosol Samplers . . . . . 69

3.1.3 Boundary Conditions, Operating Conditions, and Computational Grids . . . . . . . . . . . . . . . . . . 69

3.1.4 Use of Reference Probe in Computational Domain . . . . . . . . . 83

3.2 Resources . . . . . . . . . . . . . . . . . . . 86

3.2.1 Commercial CFD Software Package . . . . . . . . . . . . . 86

3.2.2 Graphics and Plotting Software . . . . . . . . . . . . . 86

3.2.3 Computer Hardware . . . . . . . . . . . . . . . . . . . 87

3.3 Data Analysis . . . . . . . . . . . . . . . . . . 87

3.3.1 Qualitative Plots . . . . . . . . . . . . . . 88

3.3.2 Percent Relative Difference . . . . . . . . . . . . . . . 88

3.3.3 Parameter Space Analysis . . . . . . . . . . . . . . . . . . 89

3.3.4 Grid Convergence Studies . . . . . . . . . . . . . . . . . 92

3.4 Summary of Research Methods . . . . . . . . . . . . . . . . . . . . . . 93

4 Results $\quad 95$

4.1 CFD Simulations . . . . . . . . . . . . . . . . . . 95

4.1.1 Analysis of Simulation Results for Probe and GSP Samplers . . . . 99

4.1.2 CFD Simulations Summary . . . . . . . . . . . . . . 118

4.2 Particle Trajectory Calculations of Samplers . . . . . . . . . . . . . . 119

4.2.1 GSP Personal Sampler . . . . . . . . . . . . . . . . . . . . . 124

4.2.2 IOM Inhalable Personal Sampler . . . . . . . . . . . . . . . 154 
5 Discussion and Conclusions

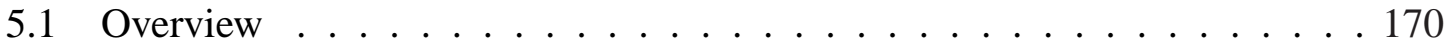

5.2 Discussion . . . . . . . . . . . . . . . . . . 172

6 References $\quad 176$

$\begin{array}{lll}\text { A Results Tables } & 191\end{array}$

$\begin{array}{lr}\text { B Computational Resources } & 218\end{array}$

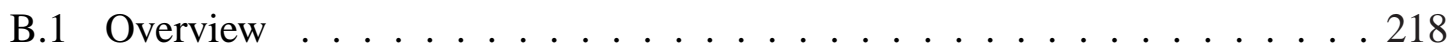

B.2 Background . . . . . . . . . . . . . . . . . . . . 219

B.3 Performance Evaluation Methodology . . . . . . . . . . . . . . . 221

B.3.1 Simulation Benchmarks . . . . . . . . . . . . 221

B.3.2 Elapsed Time and Ratings . . . . . . . . . . . . . . . . . 224

B.4 Results . . . . . . . . . . . . . . . . . . . . . 224

B.4.1 Rating . . . . . . . . . . . . . . . . . 224

B.4.2 Parallel Scaling . . . . . . . . . . . . . . . 233

B.5 Conclusions . . . . . . . . . . . . . . . . . 233 


\section{List of Figures}

1.1 This personal sampler, called the IOM Inhalable, is attached to a worker's lapel near the worker's nose and mouth, i.e. their breathing zone, for monitoring dust exposure. . . . . . . . . . . . . . 3

1.2 37-mm Plastic Closed-face Sampling Cassette. . . . . . . . . . . . 6

1.3 Dorr-Oliver style cyclone sampler. . . . . . . . . . . . . . . . . 7

1.4 Stainless steel version of the IOM Inhalable Sampler. . . . . . . . . . . 8

1.5 GSP Sampler. . . . . . . . . . . . . . . . . . . . . . . 9

1.6 Particle deposition occurring in a closed-face cassette is focused into the center of the filter by the small, high-velocity opening. Photo courtesy of Martin Harper, NIOSH. . . . . . . . . . . . . . . . . . . . . 13

1.7 Uniform particle deposition across the surface of the filter in a GSP sampler. Photo courtesy of Martin Harper, NIOSH. . . . . . . . . . . . . . 14

1.8 Inhalability penetration curves for aerosol particles entering the three primary regions of the lung (after Soderholm, 1989) . . . . . . . . . . 17

3.1 North-South-East-West grid used to demonstrate the discretizations used in CFD solvers. . . . . . . . . . . . . . . . . . 66

3.2 Dimensions of GSP sampler. . . . . . . . . . . . . . . . 73

3.3 Dimensions of IOM sampler. . . . . . . . . . . . . . . . . . . . . 74

3.4 GSP and IOM samplers free in the air at 0-degree orientations with respect to freestream airflow direction. . . . . . . . . . . . . . 75 
3.5 GSP and IOM samplers free in the air at 90-degree orientations with respect to freestream airflow direction. . . . . . . . . . . . . . . . 76

3.6 GSP and IOM samplers free in the air at 180-degree orientations with respect to freestream airflow direction. . . . . . . . . . . . 77

3.7 One-half symmetry diagram of GSP sampler mounted on the box-shaped bluff body at a 0 -degree orientation. For 90 -degree orientations, the sampler is moved to the side, and for 180-degree orientations it is moved to the back. The IOM sampler is situated in the same manner as the GSP. . . . . 78

3.8 Expected convergence behavior of scaled residuals for simulations of interest. 82

3.9 Centerline slice of 3D tetrahedral cell grid of sharp-edged probe at a 0degree orientation to oncoming freestream flow. . . . . . . . . . . . . . 84

3.10 GSP personal sampler, reference probe sampler, and ideal sampling faces. . 85

3.11 3D representation of three calculation parameters each at two levels. . . . . 91

4.1 Velocity values of three grid densities across inlet of isokinetic sampler for $0.5 \mathrm{~m} / \mathrm{s}$ freestream and sampler inlet velocities. . . . . . . . . . . 100

4.2 Velocity values of four grid densities across inlet of isokinetic sampler for $1.0 \mathrm{~m} / \mathrm{s}$ freestream and sampler inlet velocities. . . . . . . . . . . 101

4.3 Center-band and near-wall analysis locations for GSP sampler. The reference probe analyses were done for similar spatial locations. . . . . . . . . 102

4.4 Percent relative differences between 1st- and 2nd-order CFD solvers for simulations of the GSP sampler for 0.5 and $1.0 \mathrm{~m} / \mathrm{s}$ freestream windspeeds at $0-, 90-$ and 180 -degree orientations. . . . . . . . . . . . . 103

4.5 Percent relative differences between 1st- and 2nd-order CFD solvers for simulations of the reference probe for 0.5 and $1.0 \mathrm{~m} / \mathrm{s}$ freestream windspeeds at $0-, 90-$, and 180 -degree orientations. . . . . . . . . . . . 104

4.6 Plotted uncertainties determined from grid convergence studies on three grid densities of simulation results of GSP sampler at $0.5 \mathrm{~m} / \mathrm{s}$ and 0 -degree orientation. . . . . . . . . . . . . . . . . . 109 
4.7 Plotted uncertainties determined from grid convergence studies on three grid densities of simulation results of GSP sampler at $1.0 \mathrm{~m} / \mathrm{s}$ and 0 -degree orientation. . . . . . . . . . . . . . . . 110

4.8 Plotted uncertainties determined from grid convergence studies on three grid densities of simulation results of GSP sampler at $0.5 \mathrm{~m} / \mathrm{s}$ and 0 -degree orientation.

4.9 Plotted uncertainties determined from grid convergence studies on three grid densities of simulation results of GSP sampler at $1.0 \mathrm{~m} / \mathrm{s}$ and 0 -degree orientation.

4.10 Noncomforming grid elements on inside and outside surfaces of GSP sampler for turbulent $y^{+}$values before adaption. See Figure 4.11 for detailed mesh adaption inside the inlet. . . . . . . . . . . . . . . . . 114

4.11 Before and after adaption for turbulent $y^{+}$values inside the inlet of the GSP sampler. There is greater adaption on the right side because this is the upstream side of the sampler and more turbulence is present here. . . . . 115

4.12 Contour plot of turbulent reynolds number values for probe reference sampler in freestream flow of $0.5 \mathrm{~m} / \mathrm{s}$. View is looking downstream toward inlet of sampler. . . . . . . . . . . . . . . . . . 116

4.13 Contour plot of turbulent reynolds number values for probe reference sampler in freestream flow of $1.0 \mathrm{~m} / \mathrm{s}$. View is looking downstream toward inlet of sampler. . . . . . . . . . . . . . . . . . . 117

4.14 Wireframe diagram of ideal sampling faces, conical inlet section of GSP sampler, probe reference sampler, and locations of aerosol release lines. . . 120

4.15 Sampling efficiency of sharp-edged probe sampler from FLUENT calculations at freestream velocities of 0.5 and $1.0 \mathrm{~m} / \mathrm{s} \ldots \ldots . . \ldots 123$

4.16 GSP sampling efficiency values from CFD simulation and experiments by $\mathrm{Li}$ (1999) for "filter-only" sampling at an orientation of 0 degrees and a freestream windspeed of $0.5 \mathrm{~m} / \mathrm{s}$. The tuning factor from calculated sampling efficiency to corrected sampling efficiency for the CFD simulation is 0.7. . . . . . . . . . . . . . . . . . . 129 
4.17 GSP sampling efficiency values from CFD simulation and experiments by $\mathrm{Li}$ (1999) for "total" sampling (filter + losses) at an orientation of 0 degrees and a freestream windspeed of $0.5 \mathrm{~m} / \mathrm{s}$. The tuning factor from calculated sampling efficiency to corrected sampling efficiency for the CFD simula-

4.18 GSP sampling efficiency values from CFD simulation and experiments by Li (1999) for filter-only sampling at an orientation of 90 degrees and a freestream windspeed of $0.5 \mathrm{~m} / \mathrm{s}$. The tuning factor from calculated sampling efficiency to corrected sampling efficiency for the CFD simulation is 1.92

4.19 GSP sampling efficiency values from CFD simulation and experiments by Li (1999) for "total" sampling (filter + losses) at an orientation of 90 degrees and a freestream windspeed of $0.5 \mathrm{~m} / \mathrm{s}$. The tuning factor from calculated sampling efficiency to corrected sampling efficiency for the CFD simulation is $1.76 \ldots \ldots \ldots \ldots$. . . . . . . . . . . . . . . . . . . . . . . .

4.20 GSP sampling efficiency values from CFD simulation and experiments by Li (1999) for filter-only sampling at an orientation of 180 degrees and a freestream windspeed of $0.5 \mathrm{~m} / \mathrm{s}$. The tuning factor from calculated sampling efficiency to corrected sampling efficiency for the CFD simulation is 14.8

4.21 GSP sampling efficiency values from CFD simulation and experiments by $\mathrm{Li}$ (1999) for "total" sampling (filter + losses) at an orientation of $180 \mathrm{de}-$ grees and a freestream windspeed of $0.5 \mathrm{~m} / \mathrm{s}$. The tuning factor from calculated sampling efficiency to corrected sampling efficiency for the CFD simulation is 6.9 .

4.22 GSP sampling efficiency values from CFD simulation and experiments by $\mathrm{Li}$ (1999) for filter-only sampling at an orientation of 0 degrees and a freestream windspeed of $1 \mathrm{~m} / \mathrm{s}$. The tuning factor from calculated sampling efficiency to corrected sampling efficiency for the CFD simulation is 0.8 .

4.23 GSP sampling efficiency values from CFD simulation and experiments by Li (1999) for "total" sampling (filter + losses) at an orientation of 0 degrees and a freestream windspeed of $1 \mathrm{~m} / \mathrm{s}$. The tuning factor from calculated sampling efficiency to corrected sampling efficiency for the CFD simulation is 0.71 . 
4.24 GSP sampling efficiency values from CFD simulation and experiments by $\mathrm{Li}$ (1999) for filter-only sampling at an orientation of 90 degrees and a freestream windspeed of $1.0 \mathrm{~m} / \mathrm{s}$. The tuning factor from calculated sampling efficiency to corrected sampling efficiency for the CFD simulation is 6.6 .

4.25 GSP sampling efficiency values from CFD simulation and experiments by Li (1999) for "total" sampling (filter + losses) at an orientation of 90 degrees and a freestream windspeed of $1.0 \mathrm{~m} / \mathrm{s}$. The tuning factor from calculated sampling efficiency to corrected sampling efficiency for the CFD simulation is $6.7 \ldots \ldots \ldots \ldots \ldots$

4.26 GSP sampling efficiency values from CFD simulation and experiments by Li (1999) for filter-only sampling at an orientation of 180 degrees and a freestream windspeed of $1.0 \mathrm{~m} / \mathrm{s}$. The tuning factor from calculated sampling efficiency to corrected sampling efficiency for the CFD simulation is 30. . . . . . . . . . . . . . . . . . . . . 140

4.27 GSP sampling efficiency values from CFD simulation and experiments by Li (1999) for "total" sampling (filter + losses) at an orientation of 180 degrees and a freestream windspeed of $1.0 \mathrm{~m} / \mathrm{s}$. The tuning factor from calculated sampling efficiency to corrected sampling efficiency for the CFD simulation is 18.6. . . . . . . . . . . . . . . . . . . . . 141

4.28 3X direction-averaged sampling efficiency values for GSP sampler mounted on box-shaped bluff body in freestream windspeed of $0.5 \mathrm{~m} / \mathrm{s}$. . . . . . 149

4.29 Witschger direction-averaged sampling efficiency values for GSP sampler mounted on box-shaped bluff body in freestream windspeed of $0.5 \mathrm{~m} / \mathrm{s}$ with experimental values from Kenny et al. (1997). . . . . . . . . . . . . . . . 150

4.30 Witschger direction-averaged sampling efficiency values for GSP sampler mounted on box-shaped bluff body in freestream windspeeds of 1.25 and $2.0 \mathrm{~m} / \mathrm{s}$ with experimental values from Kenny et al. (1997). . . . . . . . . . 151

4.31 Sampling efficiencies of IOM sampler in comparison with experimental results from $\mathrm{Li}$ (1999) for the sampler free in the air and oriented 0 degrees to a $0.5 \mathrm{~m} / \mathrm{s}$ freestream airflow. . . . . . . . . . . . . . . . . 156

4.32 Sampling efficiencies of IOM sampler in comparison with experimental results from $\mathrm{Li}$ (1999) for the sampler free in the air and oriented 90 degrees to a $0.5 \mathrm{~m} / \mathrm{s}$ freestream airflow. 
4.33 Sampling efficiencies of IOM sampler in comparison with experimental results from Li (1999) for the sampler free in the air and oriented 180 degrees to a $0.5 \mathrm{~m} / \mathrm{s}$ freestream airflow. . . . . . . . . . . . . . . 158

4.34 Sampling efficiencies of IOM sampler in comparison with experimental results from $\mathrm{Li}$ (1999) for the sampler free in the air and oriented 0 degrees to a $1.0 \mathrm{~m} / \mathrm{s}$ freestream airflow. . . . . . . . . . . . . . . . . 160

4.35 Sampling efficiencies of IOM sampler in comparison with experimental results from $\mathrm{Li}$ (1999) for the sampler free in the air and oriented 90 degrees to a $1.0 \mathrm{~m} / \mathrm{s}$ freestream airflow. . . . . . . . . . . . . . . . . . 161

4.36 Sampling efficiencies of IOM sampler in comparison with experimental results from $\mathrm{Li}$ (1999) for the sampler free in the air and oriented 180 degrees to a $1.0 \mathrm{~m} / \mathrm{s}$ freestream airflow. . . . . . . . . . . . . . . . . 162

4.37 Witschger direction-averaged sampling efficiency values for IOM sampler mounted on box-shaped bluff body in freestream windspeed of $0.5 \mathrm{~m} / \mathrm{s}$. with experimental values from Kenny et al. (1997) . . . . . . . . . . . 166

4.38 3X direction-averaged sampling efficiency values for IOM sampler mounted on box-shaped bluff body in freestream windspeed of $0.5 \mathrm{~m} / \mathrm{s}$. with experimental values from Kenny et al. (1997) . . . . . . . . . . . . . . . . 167

4.39 Witschger direction-averaged sampling efficiency values for the IOM sampler mounted on a box-shaped bluff body in freestream windspeeds of 1.25 and $2.0 \mathrm{~m} / \mathrm{s}$ with experimental values from Kenny et al. (1997) for windspeeds of 1.0 and $4.0 \mathrm{~m} / \mathrm{s}$. . . . . . . . . . . . . . . . . . 168

B.1 Comparison of parallel scaling between SUN 6000 and Linux Networx XEON Cluster for calculations run for 1000 iterations on coarse grid size. . 226

B.2 Comparison of parallel scaling between SUN 6000 and Linux Networx XEON Cluster for calculations run for 1000 iterations on medium grid size. 228

B.3 Comparison of parallel scaling between SUN 6000 and Linux Networx XEON Cluster for calculations run for 1000 iterations on fine grid size. . . 229

B.4 Comparison of parallel scaling between SUN 6000 and Linux Networx XEON Cluster for calculations run for 601 iterations on fine grid size. . . . 232 


\section{List of Tables}

1.1 Wind tunnel and mannequin sizes for selected studies of sampling efficiency. 21

3.1 Low- and high-level coding map for parameter space analysis. . . . . . . . 90

4.1 Calculation parameters and levels used for CFD simulations in this work. . 97

4.2 Additional calculation parameters and levels for CFD simulations. . . . . . 98

4.3 Values determined from parameter space analysis to yield $10 \%$ or greater influence on desired results are shown in bold. . . . . . . . . . . . . . 106

4.4 Correction tuning factors for calculated sampling efficiencies of the GSP sampler based on experimental values from $\mathrm{Li}$ (1999) together with the resulting average percent relative disagreement for that condition. . . . . . 143

4.5 Average correction tuning factors for the GSP sampler at 0-, 90-, and 180degree orientations to freestream flows of 0.5 and $1.0 \mathrm{~m} / \mathrm{s}$ for filter-only sampling with deviation from original values of relative percent disagreement in parentheses. . . . . . . . . . . . . . . . . . . . . 144

4.6 Correction tuning constants, based on Inhalability convention, and the operation function on their respective data sets from simulations of the GSP sampler mounted on the box-shaped bluff body with experimental values from Kenny et al. (1997). . . . . . . . . . . . . . . . . . . . . . 152

4.7 Percent relative disagreement of Witschger averaged simulation results for the GSP sampler at $0.5 \mathrm{~m} / \mathrm{s} \ldots \ldots \ldots 153$

4.8 Correction tuning factors based on experimental values from Li (1999) and the resulting average percent relative disagreement for that condition. . . . 163 
4.9 Average correction tuning factors for 0-, 90-, and 180-degree orientations to freestream flows of 0.5 and $1.0 \mathrm{~m} / \mathrm{s}$ for IOM sampler with deviation from original values of relative percent disagreement in parentheses. . . . . . . 164

4.10 Correction tuning constants, based on Inhalability convention, and the operation function on their respective data sets from simulations of the IOM sampler mounted on the box-shaped bluff body. . . . . . . . . . . . 169

A.1 Calculated and corrected "filter-only" sampling efficiencies from CFD simulation of the GSP sampler free in the air at a 0-degree orientation to $0.5 \mathrm{~m} / \mathrm{s}$ freestream airflow with corresponding experimental data from ( $\mathrm{Li}, 1999)$. See Figure 4.16. . . . . . . . . . . . . . . . . . . . . . 192

A.2 Calculated and corrected "total" sampling efficiencies from CFD simulation of the GSP sampler free in the air at a 0-degree orientation to $0.5 \mathrm{~m} / \mathrm{s}$ freestream airflow with corresponding experimental data from ( $\mathrm{Li}, 1999)$. See Figure 4.17 . . . . . . . . . . . . . . . . . . . . 193

A.3 Calculated and corrected "filter-only" sampling efficiencies from CFD simulation of the GSP sampler free in the air at a 90-degree orientation to $0.5 \mathrm{~m} / \mathrm{s}$ freestream airflow with corresponding experimental data from $(\mathrm{Li}$, 1999). See Figure 4.18. . . . . . . . . . . . . . . . . . . 194

A.4 Calculated and corrected "total" sampling efficiencies from CFD simulation of the GSP sampler free in the air at a 90-degree orientation to $0.5 \mathrm{~m} / \mathrm{s}$ freestream airflow with corresponding experimental data from $(\mathrm{Li}, 1999)$. See Figure 4.19 . . . . . . . . . . . . . . . . . . . . . . 195

A.5 Calculated and corrected "total" sampling efficiencies from CFD simulation of the GSP sampler free in the air at a 180-degree orientation to $0.5 \mathrm{~m} / \mathrm{s}$ freestream airflow with corresponding experimental data from $(\mathrm{Li}, 1999)$. See Figure 4.20 . . . . . . . . . . . . . . . . . 196

A.6 Calculated and corrected "total" sampling efficiencies from CFD simulation of the GSP sampler free in the air at a 180-degree orientation to $0.5 \mathrm{~m} / \mathrm{s}$ freestream airflow with corresponding experimental data from ( $\mathrm{Li}, 1999)$. See Figure 4.21 . . . . . . . . . . . . . . . . . . . . . . . . . . 197 
A.7 Calculated and corrected "filter-only" sampling efficiencies from CFD simulation of the GSP sampler free in the air at a 0-degree orientation to $1.0 \mathrm{~m} / \mathrm{s}$ freestream airflow with corresponding experimental data from $(\mathrm{Li}, 1999)$. See Figure 4.22 . . . . . . . . . . . . . . . . . . . . . . . 198

A.8 Calculated and corrected "total" sampling efficiencies from CFD simulation of the GSP sampler free in the air at a 0-degree orientation to $1.0 \mathrm{~m} / \mathrm{s}$ freestream airflow with corresponding experimental data from $(\mathrm{Li}, 1999)$. See Figure 4.23 . . . . . . . . . . . . . . . . . . . . . . . . . 199

A.9 Calculated and corrected "filter" sampling efficiencies from CFD simulation of the GSP sampler free in the air at a 90-degree orientation to $1.0 \mathrm{~m} / \mathrm{s}$ freestream airflow with corresponding experimental data from (Li, 1999). See Figure 4.24 . . . . . . . . . . . . . . . . . . . . 200

A.10 Calculated and corrected "total" sampling efficiencies from CFD simulation of the GSP sampler free in the air at a 90-degree orientation to $1.0 \mathrm{~m} / \mathrm{s}$ freestream airflow with corresponding experimental data from ( $\mathrm{Li}, 1999)$. See Figure 4.25 . . . . . . . . . . . . . . . . . . . . 201

A.11 Calculated and corrected "filter" sampling efficiencies from CFD simulation of the GSP sampler free in the air at a 180-degree orientation to $1.0 \mathrm{~m} / \mathrm{s}$ freestream airflow with corresponding experimental data from $(\mathrm{Li}, 1999)$.

See Figure 4.26. . . . . . . . . . . . . . . . . . . . . . . 202

A.12 Calculated and corrected "total" sampling efficiencies from CFD simulation of the GSP sampler free in the air at a 180-degree orientation to $1.0 \mathrm{~m} / \mathrm{s}$ freestream airflow with corresponding experimental data from (Li, 1999). See Figure 4.27 . . . . . . . . . . . . . . . . . . . . . 203

A.13 Calculated and corrected " $3 \mathrm{X}$ averaging" sampling efficiencies from CFD simulation of the GSP sampler mounted on a box-shaped bluff body in 0.5 $\mathrm{m} / \mathrm{s}$ freestream airflow with corresponding experimental data from (Kenny et al. 1997). See Figure 4.28. . . . . . . . . . . . . . . . . . . . . . 204

A.14 Calculated and corrected "Witschger averaging" sampling efficiencies from CFD simulation of the GSP sampler mounted on a box-shaped bluff body in $0.5 \mathrm{~m} / \mathrm{s}$ freestream airflow with corresponding experimental data from (Kenny et al. 1997). See Figure 4.29. 
A.15 Sampling efficiency values from (Kenny et al. 1997) for the GSP sampler mounted on a step-wise rotating mannequin in a wind tunnel. See Figure

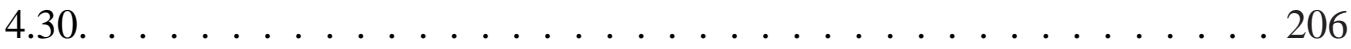

A.16 Calculated and corrected " $3 X$ " and "Witschger" averaged sampling efficiency values from CFD simulation of the GSP sampler mounted on a boxshaped bluff body in 1.25 and 2.0 m/s freestream airflow. See Figure 4.30. . 207

A.17 Calculated and corrected "total" sampling efficiencies from CFD simulation of the IOM sampler free in the air at a 0-degree orientation to $0.5 \mathrm{~m} / \mathrm{s}$ freestream airflow with corresponding experimental data from $(\mathrm{Li}, 1999)$. See Figure 4.31 . . . . . . . . . . . . . . . . . . . . . 208

A.18 Calculated and corrected "total" sampling efficiencies from CFD simulation of the IOM sampler free in the air at a 90-degree orientation to $0.5 \mathrm{~m} / \mathrm{s}$ freestream airflow with corresponding experimental data from $(\mathrm{Li}, 1999)$. See Figure 4.32 . . . . . . . . . . . . . . . . . . . . . . . 209

A.19 Calculated and corrected "total" sampling efficiencies from CFD simulation of the IOM sampler free in the air at a 180-degree orientation to $0.5 \mathrm{~m} / \mathrm{s}$ freestream airflow with corresponding experimental data from (Li, 1999). See Figure 4.33 . . . . . . . . . . . . . . . . . . . . . . 210

A.20 Calculated and corrected "total" sampling efficiencies from CFD simulation of the IOM sampler free in the air at a 0-degree orientation to $1.0 \mathrm{~m} / \mathrm{s}$ freestream airflow with corresponding experimental data from $(\mathrm{Li}, 1999)$. See Figure 4.34 . . . . . . . . . . . . . . . . . . . 211

A.21 Calculated and corrected "total" sampling efficiencies from CFD simulation of the IOM sampler free in the air at a 90-degree orientation to $1.0 \mathrm{~m} / \mathrm{s}$ freestream airflow with corresponding experimental data from (Li, 1999).

See Figure 4.35 . . . . . . . . . . . . . . . . . . . . 212

A.22 Calculated and corrected "total" sampling efficiencies from CFD simulation of the IOM sampler free in the air at a 180-degree orientation to $1.0 \mathrm{~m} / \mathrm{s}$ freestream airflow with corresponding experimental data from $(\mathrm{Li}, 1999)$. See Figure 4.36 . . . . . . . . . . . . . . . . . . . . 213

A.23 Calculated and corrected "Witschger" averaged sampling efficiencies from CFD simulation of the IOM sampler mounted on a box-shaped bluff body in $0.5 \mathrm{~m} / \mathrm{s}$ freestream airflow with corresponding experimental data from (Kenny et al. 1997). See Figure 4.37. 
A.24 Calculated and corrected " $3 X$ " sampling efficiencies from CFD simulation of the IOM sampler mounted on a box-shaped bluff body in $0.5 \mathrm{~m} / \mathrm{s}$ freestream airflow with corresponding experimental data from (Kenny et al. 1997). See Figure 4.38. . . . . . . . . . . . . . . . . . . 215

A.25 Sampling efficiency values from (Kenny et al. 1997) for the IOM sampler mounted on a step-wise rotating mannequin in a wind tunnel. See Figure 4.39. . . . . . . . . . . . . . . . . . . 216

A.26 Calculated and corrected " $3 X$ " and "Witschger" averaged sampling efficiency values from CFD simulation of the IOM sampler mounted on a box-shaped bluff body in 1.25 and $2.0 \mathrm{~m} / \mathrm{s}$ freestream airflow. See Figure 4.39.

B.1 Boundary conditions, grid density, and iterations for simulation benchmarks used in this performance evaluation. . . . . . . . . . . 223

B.2 Results of simulation benchmarks for the SUN 6000 and linux cluster calculations of CFD models at medium (0.001) and coarse (0.0015) grid sizes. 227

B.3 Results of simulation benchmarks for SUN 6000 calculations of CFD models at the fine grid size $(0.0005) . \ldots \ldots 230$

B.4 Results of simulation benchmarks for linux cluster calculations of CFD models at the fine grid size $(0.0005) \ldots \ldots . \ldots . \ldots . \ldots . \ldots 231$

B.5 Results of efficiency determinations for the XEON linux cluster. . . . . . . 234

B.6 Results of efficiency determinations for the SUN 6000. . . . . . . . . . . 235 


\section{Chapter 1}

\section{Introduction}

Workplaces in the United States are mandated by Federal regulations to maintain safe and healthful working conditions for all employees. In 1970, the United States Congress passed the Occupational Safety and Health Act, "to assure so far as possible every working man and woman in the Nation safe and healthful working conditions and to preserve our human resources" (United States Code, 29 USC 651). These regulations were made based on 40 years of labor statistics showing that a great many workplaces were dangerous and life threatening to workers (U.S. Dept. of Labor, Bureau of Labor Statistics, 2002).

The Federal regulations provide guidelines for control of a large number of workplace hazards, and of these hazards, airborne dust contaminants receive a great deal of attention in the law. In fact, dust is directly responsible for an estimated 31,000 deaths over the past ten years (NIOSH, 2003), and is associated with complications in the deaths of at least 4,000 workers each year. The largest source of these numbers in the United States can be attributed to dust exposures caused by surface and subsurface mining machines. In addition, 
there are many more sources of dust exposure including, welding and pipe cutting (NIOSH, 2003), lead foundry work, asbestos mitigation, brick and cement work, abrasive blasting (OSHA, 2004 $4^{a}$, and many other occupations. Therefore, controlling workers' exposures to airborne dust contaminants is an extremely important concern in many workplaces.

In order to monitor workers' exposures to potentially harmful dusts, industrial hygienists and other industrial safety practitioners use personal aerosol samplers to collect a sample of airborne particles. The collected material is analyzed and an assessment is made of the working environment. Figure 1.1 shows a personal aerosol sampler used to monitor a worker's exposure.

There are two types of sampling used to make determinations of airborne particulate concentrations: area sampling and personal sampling. Both types may be useful in determining the level of airborne particulate concentration. However personal sampling is more commonly used to make judgments on potential health effects due to particles in the air. In addition, personal sampling is used as a regulatory tool in enforcement of the permissible exposure limits of aerosols by the Occupational Safety and Health Administration (OSHA). Thus there is a strong motivation for Industrial Hygienists to use this method when evaluating workplace exposures.

Personal sampling of aerosols has been a required part of exposure monitoring programs since the inception of the Occupational Safety and Health Act of 1970. Control of certain aerosols can result in reduction of adverse respiratory health effects such as cancers or 


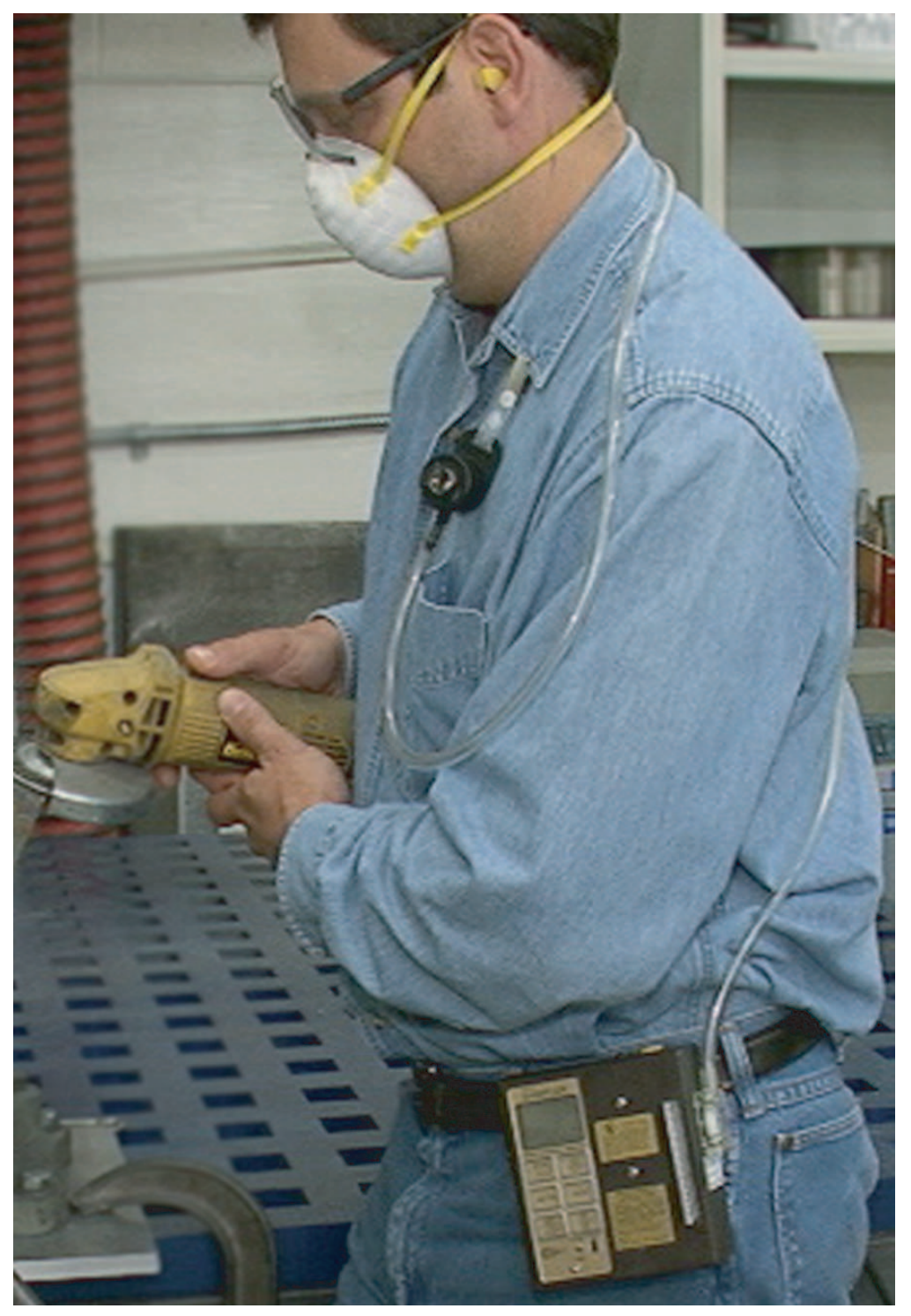

Figure 1.1: This personal sampler, called the IOM Inhalable, is attached to a worker's lapel near the worker's nose and mouth, i.e. their breathing zone, for monitoring dust exposure. 
asthma. Thus an accurate measurement of the type and amount of the aerosol is extremely important in determining the likelihood of an adverse health effect resulting from exposure to an airborne variety of a particular toxic material. In addition, determining the concentration of the dust allows the industrial hygienist and management to know how much the exposure should be reduced to in order to maintain a healthful working environment.

To measure airborne concentrations, air is drawn through the sampler by a sampling pump operating at a calibrated flow rate so particles in the vicinity of the sampler are aspirated and, ideally, deposited on a collection media such as a filter. The particles are then analyzed by a variety of methods such as weighing, X-ray diffraction, spectrophotometry, or counting. The particulate material is identified by the analysis technique and the mass can be determined as an indication of the airborne particle concentration. This in turn is used to make an assessment of potential toxicological effects or to determine degree of compliance with governmental regulations.

Many of the harmful substances found as aerosols in the workplace have exposure limits. These are values of mass concentration of substance per unit volume of air. Silica dust in quartz form, for example, has a permissible exposure limit, set by OSHA, of $30 \mathrm{mg} / \mathrm{m}^{3}$ for total dust exposure (OSHA, 2004 ${ }^{b}$ ) as determined by sampling for 8 hours using the OSHA sampling method for total dust, which is use of $37-\mathrm{mm}$ closed-face cassette operated at 2 $\mathrm{L} / \mathrm{min}$. OSHA also has a permissible exposure limit of $10 \mathrm{mg} / \mathrm{m}^{3}$ for the respirable portion of crystalline silica dust (particles $<10 \mu \mathrm{m}$ in aerodynamic diameter) for which a cyclone sampler collects the dust. There has been debate on the validity of the number chosen 
for the exposure limit. However, these values are based primarily on toxicological and epidemiological data, and are thus believed to provide a satisfactory measure of protection for a worker.

The most commonly used sampler in the United States is the $37-\mathrm{mm}$ sampling cassette with a 4-mm inlet (see Fig. 1.2). This sampler is referred to as a "total" dust sampler because it collects particles of variable diameters, typically from 1 to $100 \mu \mathrm{m}$ in diameter, and does not distinguish between particle sizes. On the other hand, the cyclone sampler does distinguish between particle sizes, removing large particles from the sampled dust so that only the smaller particles are collected (see Fig. 1.3). The 37-mm closed-face-cassette sampler has been used since at least 1963 (Millipore, Inc., 2004) and was semi-officially adopted as the preferred sampler for sampling total dust in 1970 when Congress passed the OSH Act. The cyclone sampler is used for regulatory purposes to sample respirable dust..

Additionally, there are other samplers used for evaluating workplace concentrations. Two important samplers are the Institute of Occupational Medicine (IOM) Inhalable sampler (see Fig. 1.4, at 2.0 1/min ) and the GSP sampler (in German, Gesamtstaub-Probenahmesystem, see Fig. 1.5 , at $3.51 / \mathrm{min}$ ). Both the IOM Inhalable Sampler, developed by the Institute of Occupational Medicine, Edinburgh, Scotland (Mark and Vincent, 1986), and the GSP sampler, developed by the BG-Institute for Occupational Safety and Health -BIA-, Sankt Augustin, Germany, were constructed for sampling the size range of particles expected to penetrate into worker's airways (Berges, 2004). They were both found to show similar results to the inhalability curve (Kenny et al., 1997). 


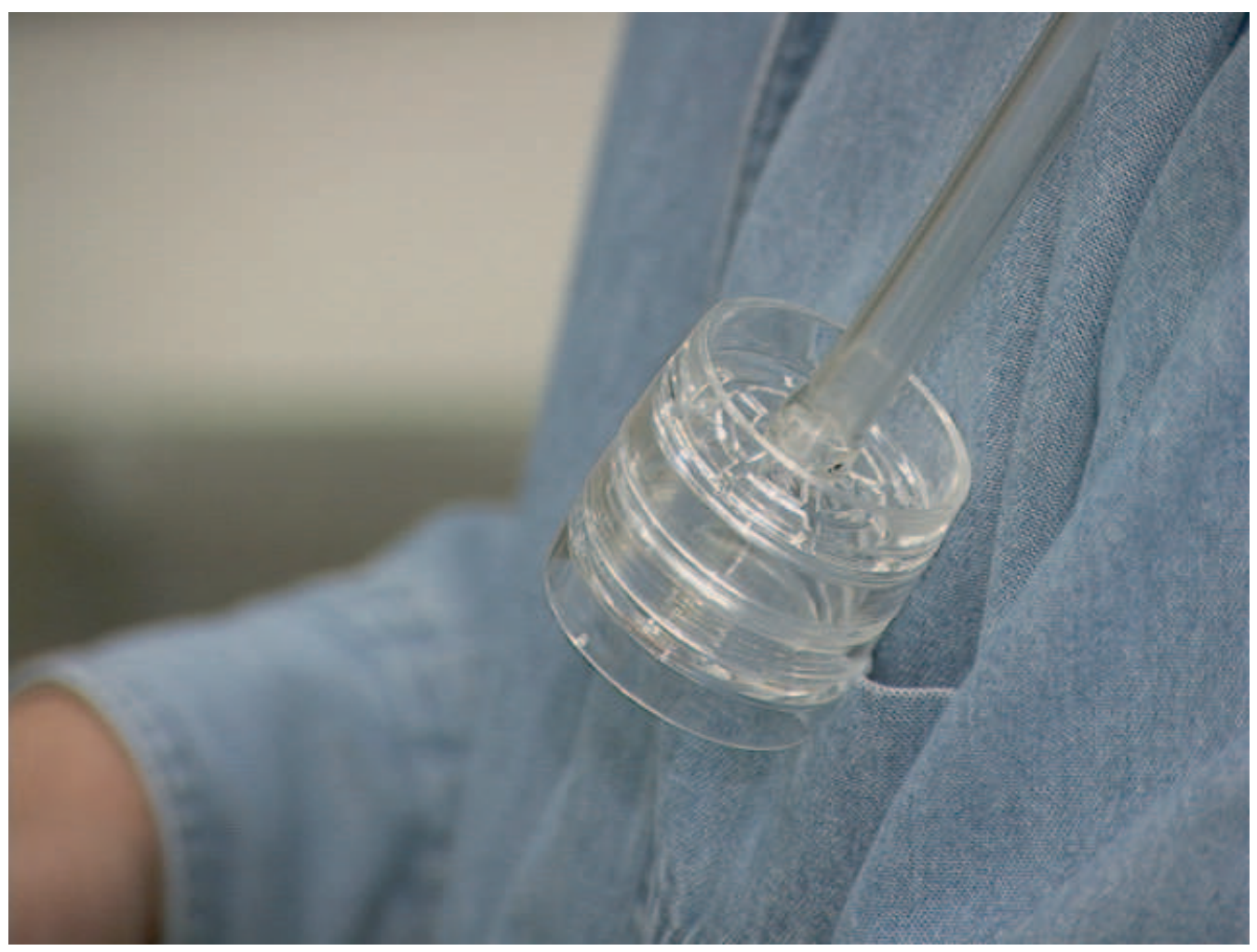

Figure 1.2: 37-mm Plastic Closed-face Sampling Cassette. 


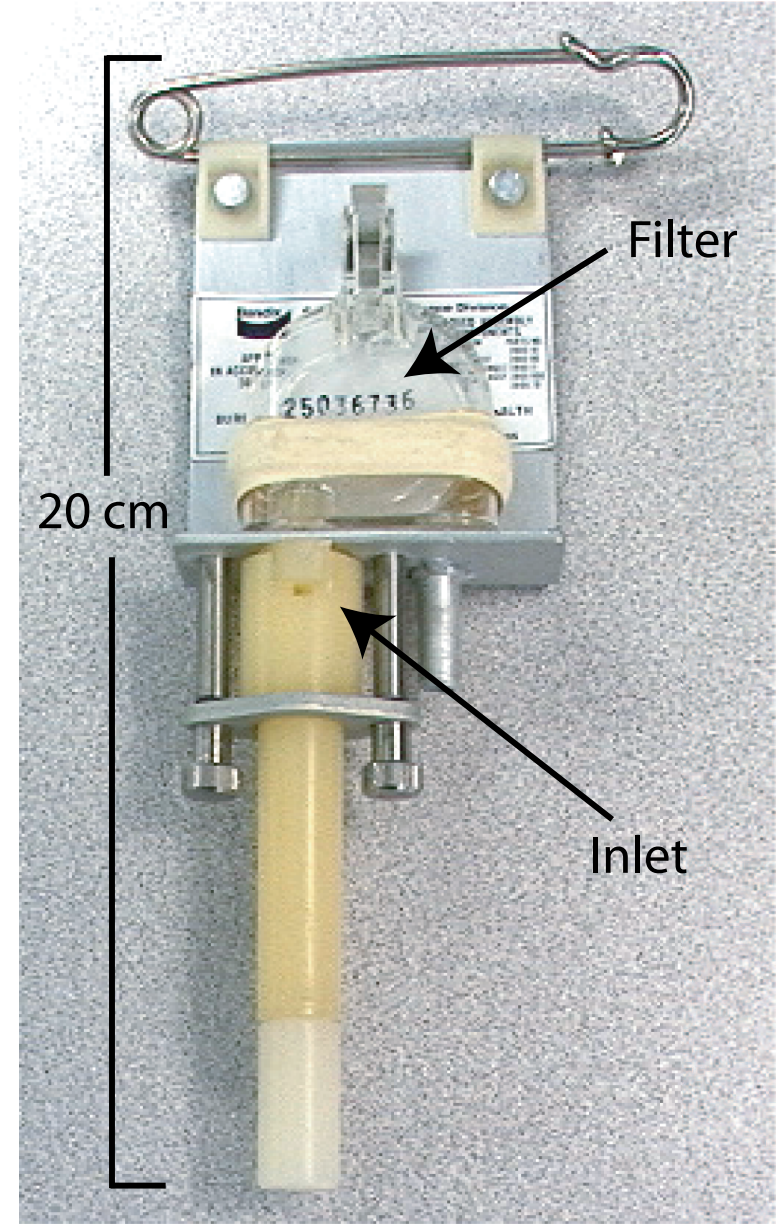

Figure 1.3: Dorr-Oliver style cyclone sampler. 


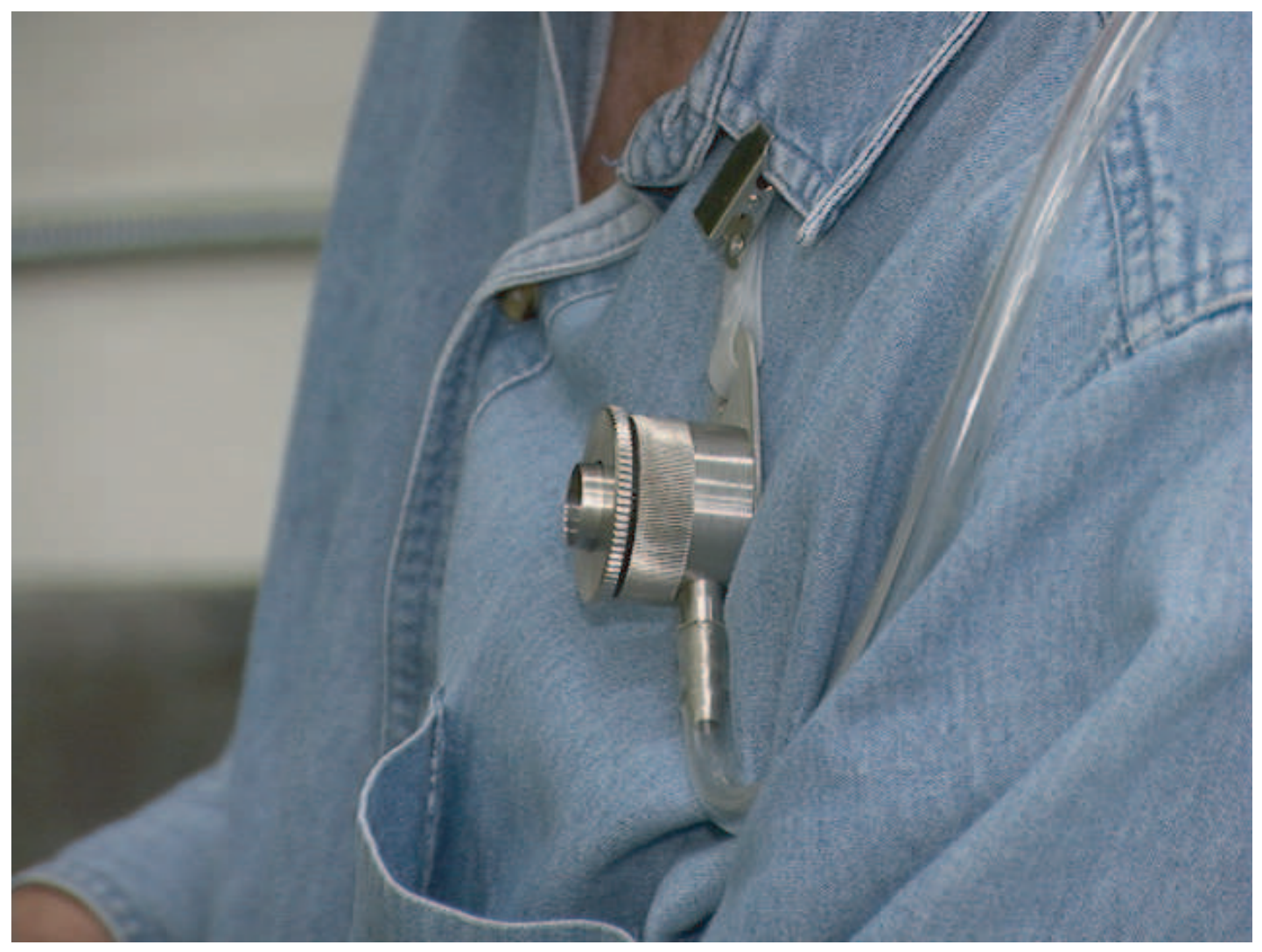

Figure 1.4: Stainless steel version of the IOM Inhalable Sampler. 


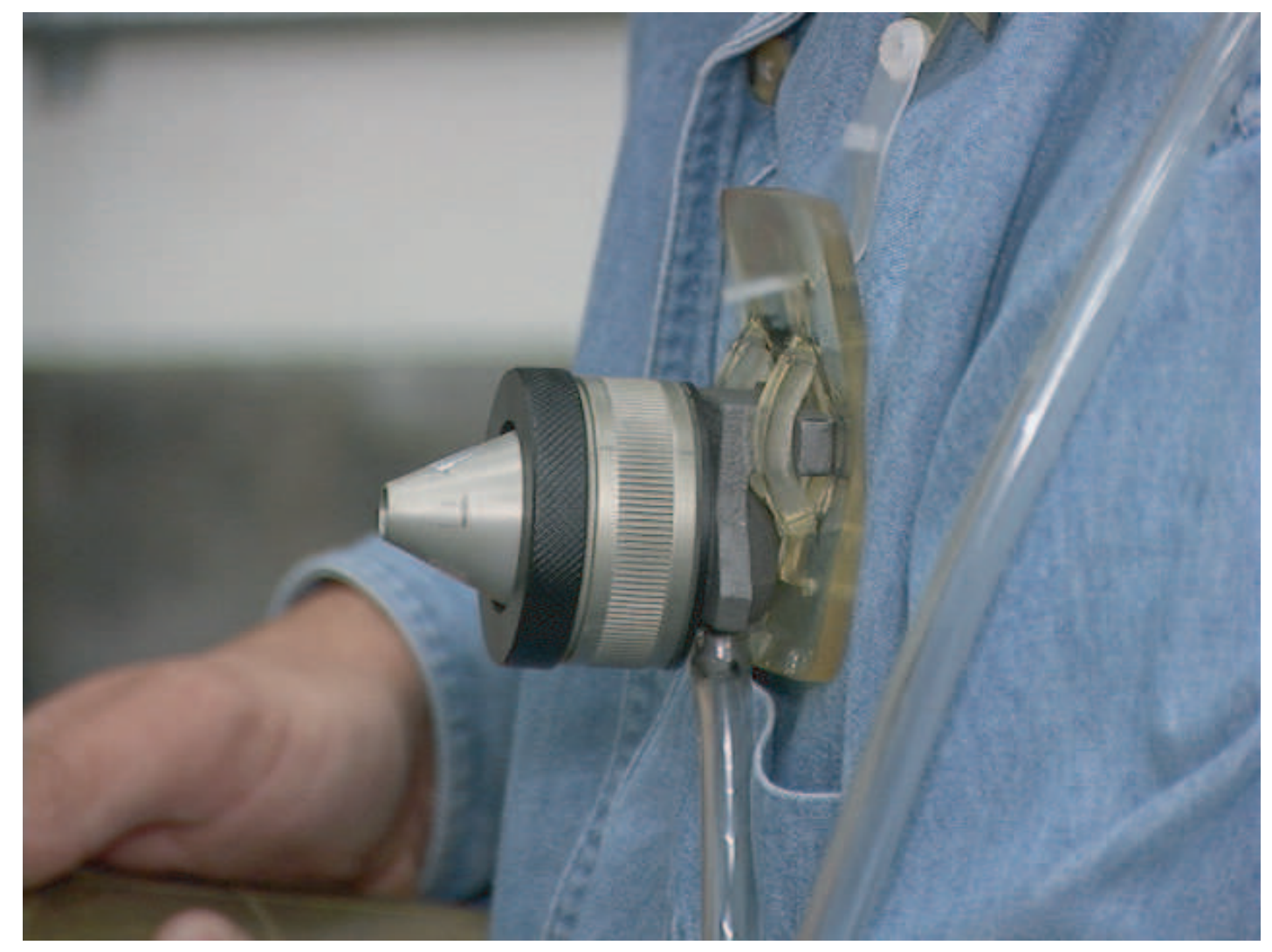

Figure 1.5: GSP Sampler. 
Proper use of the sampler and correct analysis of the filter and collected particles are essential in ensuring that a true evaluation is made of the airborne particle concentration in the workplace. Too often, samplers are assumed to efficiently collect all particles sizes, function properly in all conditions, and accurately provide a measure of mass concentration. This is, unfortunately, not true.

A lengthy list of research indicates there are many factors that contribute to the sampling efficiency of air samplers, which is defined as the ratio of concentration "seen" by an aerosol sampler to the true concentration. Most of the research results show wide variability of the apparent measure of airborne dust concentration due to the many factors involved. For example, the angle of the sampler's inlet with respect to wind flow pattern has been shown to have an effect on sampling accuracy (Chen and Baron, 1996). When the inlet is oriented directly in line with the direction of an external (freestream) particle-laden flow, then a condition of isoaxial sampling results. However, when the sampler's aspirating inlet is moved away from the oncoming flow, then a condition of non-isoaxial sampling results. In this case, the inlet velocity of the sampler must compete with the freestream velocity in order to capture the particles, which results in a change in the collection efficiency.

Sampler placement on the worker's torso may also have an effect on the ability of the sampler to collect particles since concentrations of airborne particulate matter may change depending on orientation of the worker's breathing zone (Flynn and Miller, 1991). Samplers placed within and near to the worker's breathing zone will give results more consistent with the sampling efficiency criteria established by the American Conference of Govern- 
mental Industrial Hygienists (ACGIH) and Committee for European Normalisation (CEN) of standards. Placement of the sampler nearer to the shoulder or the arm may give results different than if the sampler is placed nearer to the neck and collarbone, which is considered to be in the 0.3 meter (one foot) radius around the nose and mouth, i.e. the breathing zone.

The material a sampler is constructed from may also have an effect on sampling efficiency. Some samplers are made of plastic which can carry electrostatic charges and may disrupt the sampling efficiency by causing particles to adhere to the walls of the sampler rather than collect on the filter (Puskar et al., 1991). For example, the 37-mm closed-face cassette (CFC), commonly used for regulatory sampling in the United States, is constructed from clear plastic that is known to accumulate electrostatic charge. Charged particles, particularly in dry air, can be attracted to the surface of the sampler rather than transported to the filter by the airflow. Collection efficiency of the sampler can be subsequently decreased by this effect and due to the problem of measuring the static charge on the sampler, it has proven difficult to predict the amount and size of particles that will adhere to the sampler. Some samplers have been designed to have a decreased and/or dissipating static charge and these include the 25-mm fiber sampling cassette and the plastic IOM Inhalable Sampler. These samplers contain more elemental carbon within the plastic in order to be conductive. In addition, there are stainless steel samplers including the IOM Inhalable and the Button sampler that also dissipate the static charge.

Very important to the efficient collection of particles is the geometry of the sampling 
device. For example, the 37-mm CFC has a 4-mm inlet that opens directly into a chamber $35-\mathrm{mm}$ across with a height of $20 \mathrm{~mm}$. Since the $37-\mathrm{mm} \mathrm{CFC}$ is operated at $2 \mathrm{~L} / \mathrm{min}$, the air velocity entering the sampler is approximately $2.5 \mathrm{~m} / \mathrm{s}$. This is significantly higher than the airflow velocity found in most workplaces, which is on the order of $0.3 \mathrm{~m} / \mathrm{s}$ (Baldwin and Maynard, 1998) and results in impaction of particles in a small region on the center of the filter (see Figure 1.6).

Like the 37-mm CFC, the GSP sampler has a small inlet $(7 \mathrm{~mm})$ and a wide chamber. However, the geometry of the GSP sampler is tapered from the inlet to the filter where the diameter increases to $35 \mathrm{~mm}$. The GSP is longer than the $37-\mathrm{mm}$ CFC so the distance, and thus the time, for particles to "relax" into the changing flow conditions is greater. Studies have shown that particle deposition across the filter in a GSP sampler is relatively uniform (Harper, 2004; Figure 1.7), but there are still significant wall losses for particle sizes larger than $40 \mu m(\mathrm{Li}, 1999)$.

The 37-mm CFC, which has been used since the early 1960s (Millipore, 2004), was designed simply to collect particles from dusty workplaces. Thus, its aspiration characteristics were never intended to match inhalability characteristics of humans even though the concentration seen by the 37-mm CFC has been compared to epidemiological data, as have most samplers, for baseline exposure purposes. The GSP sampler was developed by the BG-Institute for Occupational Safety and Health -BIA-, Sankt Augustin, Germany, in order to sample the size range of particles expected to penetrate into workers' airways.

Over the past two decades, several samplers have been designed to collect particles with 


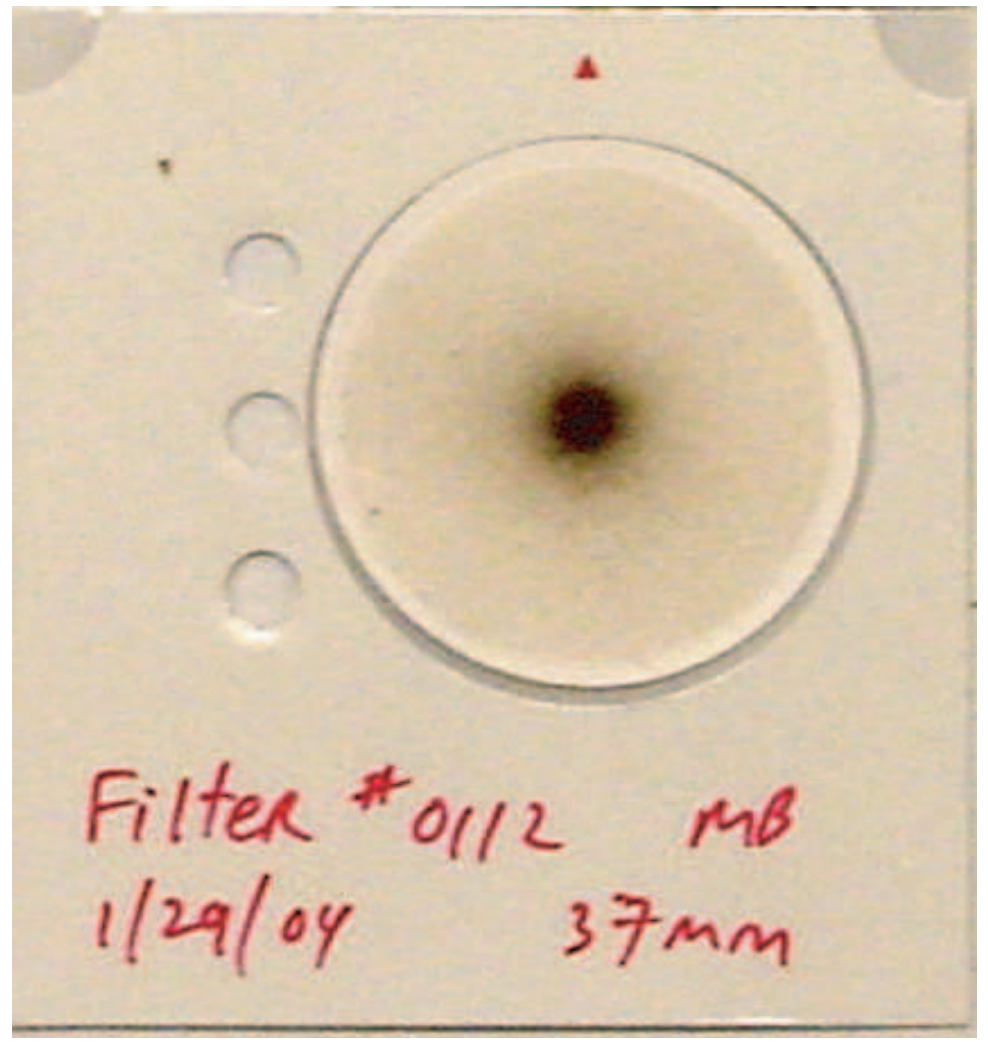

Figure 1.6: Particle deposition occurring in a closed-face cassette is focused into the center of the filter by the small, high-velocity opening. Photo courtesy of Martin Harper, NIOSH. 


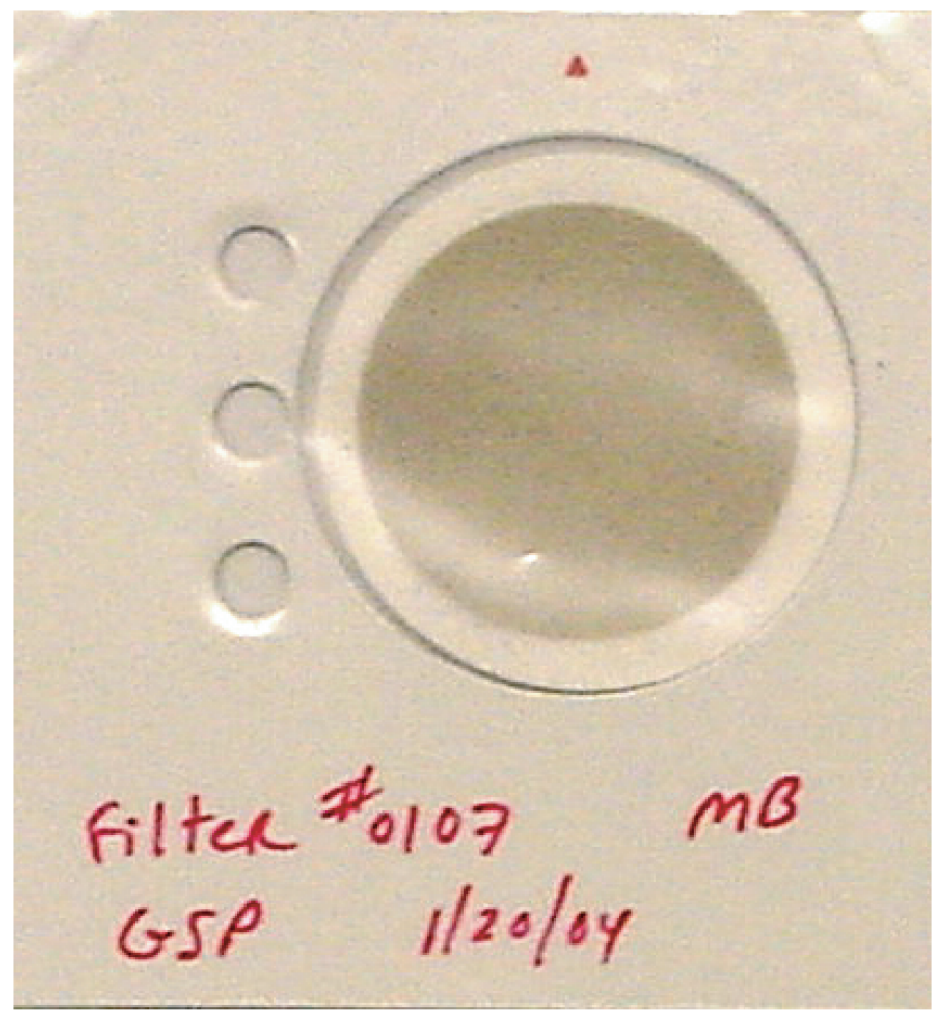

Figure 1.7: Uniform particle deposition across the surface of the filter in a GSP sampler. Photo courtesy of Martin Harper, NIOSH. 
the same efficiency as breathing models of the human head. In 1986, Mark and Vincent presented a new sampler that had been designed with wind tunnel studies to collect the inhalable fraction of particles, which was based on the performance of the aspiration of breathing human-like mannequins from wind tunnel experiments conducted by Ogden and Birkett (1977).

They called the new sampler the IOM, after the laboratory where the work was conducted, the Institute for Occupational Medicine in Edinburgh, Scotland. This sampler was designed with a large opening $(15 \mathrm{~mm})$ and a short, sharp-edged inlet. Furthermore, the sampler was designed so the entire inlet and filter holder assembly could be weighed together, which resulted in easier usage by the industrial hygienist since wall losses do not need to be measured separately.

The original version was made from stainless steel, however, for production purposes, the commercial version was made from conductive plastic. This version was found to have a greatly fluctuating mass due to the plastic's affinity for atmospheric water (Smith et al., 1998). Therefore, the plastic inlet and filter holder assembly was replaced with one made from stainless steel, and that is now the preferred way of using this sampler, which has gained wide renown and acceptance in the field of aerosol sampling.

The introduction of the IOM Inhalable sampler in 1986 was a progressive step forward because it related the performance of a sampling device with the expected sampling efficiency of a breathing worker. Even though the IOM sampler was based on an inhalable efficiency curve backed up by extensive experimentation, the acceptance of universal in- 
halability curves by all interested parties had not completely taken hold. Rather, several organizations had begun to develop their own interpretations.

Recognizing the difficulties of varying performance curves for essentially the same set of environmental conditions, Soderholm (1989), in an effort to standardize the collection of workplace aerosols in relation to an international convention, presented two curves representing particle size-selective sampling for thoracic and respirable fractions of aerosols. The new efficiency curves were based on the Inhalability curve (Vincent and Armbruster, 1981), which describes all particles that enter the human airway. The thoracic curve described those particles that penetrated into and beyond the thoracic region of the airway, and the respirable curve described those particles that penetrated into the gas-exchange region of the airway (see Figure 1.8).

These curves resulted from the combination of several organizations' and researchers' claims of the lung's ability to clear particles of varying sizes based on experimental studies of particle penetration into the human airway. From the data, which Soderholm (1989) claimed was reasonably similar, a conservative estimate of the amount and size of particles entering (penetrating into) the naso-pharyngeal region of the human head was made and is now expressed as the Inhalability convention. The combined curves of the convention received wide acceptance and were adopted by a number of international organizations including CEN (Comite' Europeen de Normalisation, 1992), International Standards Organization (ISO, 1995), and the ACGIH, reported in Vincent (1999).

Since then, some research has shown that workplace air is actually closer to calm-air 


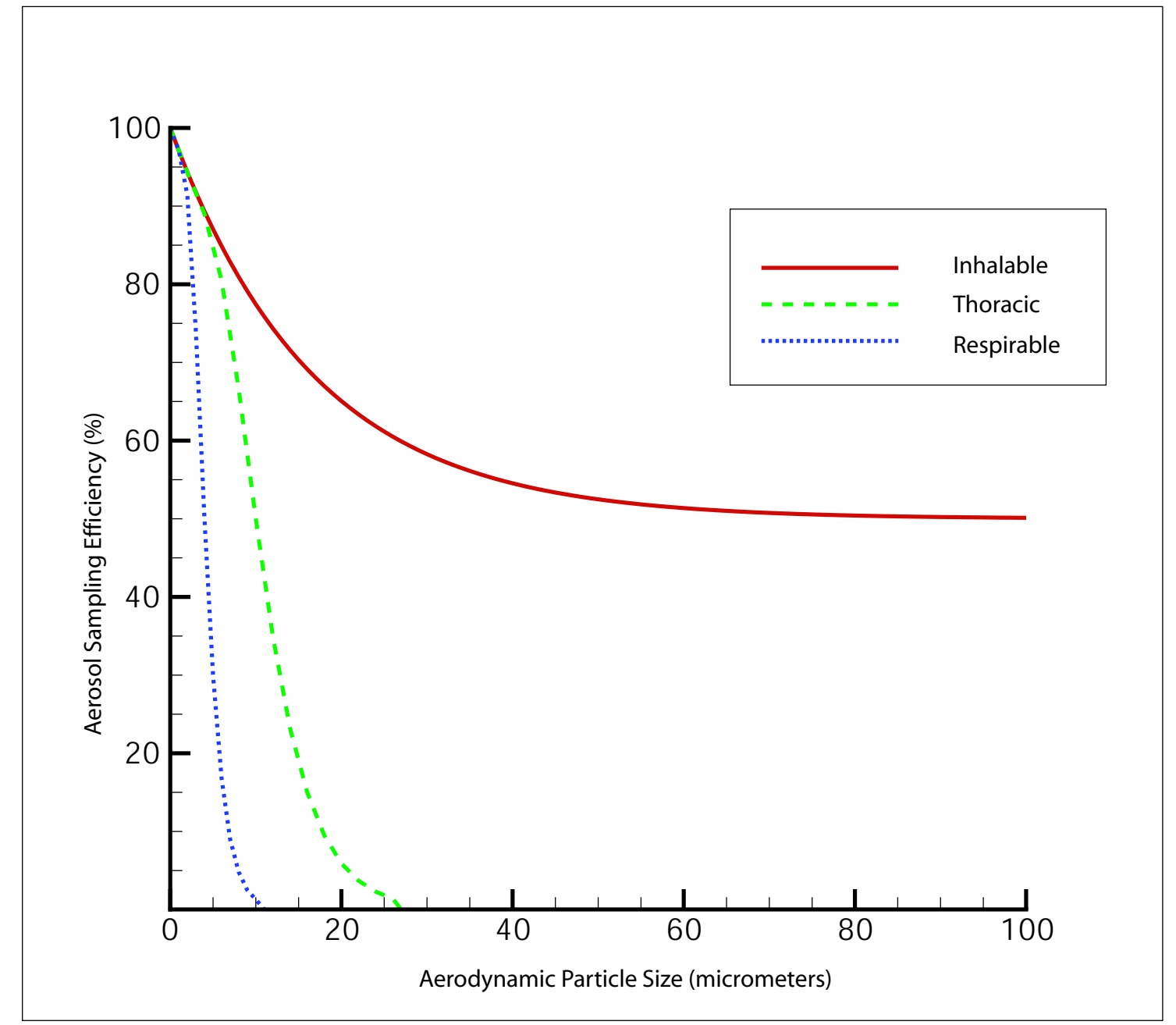

Figure 1.8: Inhalability penetration curves for aerosol particles entering the three primary regions of the lung (after Soderholm, 1989). 
conditions than to the directionally dominated windy conditions in which the Inhalability curve was originally developed. Baldwin and Maynard (1998) measured workplace air in 55 localities and found an average of $0.3 \mathrm{~m} / \mathrm{s}$, which was lower than the value presented in Ogden and Birkett (1977) of $0.75 \mathrm{~m} / \mathrm{s}$. However, in the following year, Ogden and Birkett (1978) reported efficiency measurements in a calm-air environment for a breathing mannequin that were similar to those collected in their wind tunnel study reported in 1977, which supported the application of the Inhalability convention for low-velocity airflow workplaces as well as for high-velocity workplaces.

More recently, however, researchers have conducted sampling efficiency measurements in calm-air environments that do not agree well with the Inhalability convention curve. Aitken et al. (1999) and Kenny et al. (1999) in related studies, reported the results from inhalability of a breathing mannequin and sampling efficiency tests for four samplers, respectively, in a calm-air chamber. The sampling efficiency and breathing mannequin results were close to one another for the IOM sampler, and overall the experiments were shown to have less variability in error than previously conducted work.

The resulting performance plots of the inhalability experiments and IOM measurements were typically higher than the convention curve by as much as twenty percent. This remained relatively consistent up to the largest particle sizes tested of $90 \mu \mathrm{m}$. Furthermore, the general trend of the sampling efficiency measured in the calm-air chamber was linear while the convention curve exponentially decreases until it levels off at $50 \mu \mathrm{m}$. The implications of this work are to suggest that samplers may be evaluated for low-airflow 
workplaces with a different criteria than those suggested by Ogden and Birkett (1977), Mark and Vincent (1986), and Vincent (1999).

Sampling efficiency studies have traditionally been conducted in wind tunnels and calmair chambers for the simple reason that, in them, variables in experiments can be better controlled. Since complete knowledge of the workplace environment is extremely difficult to come by, the most important and most influential factors from the workplace that effect sampling efficiency must be identified through observation. These factors are then evaluated one-by-one in the controlled laboratory studies and in simulations. Changes to the controlled environment can then be documented to determine if there has been an effect on the sampling efficiency from a particular factor or combination of factors, and by this method, the understanding of the air sampler's performance is improved.

The bulk of sampling efficiency research has been conducted in wind tunnels, for example: Badzioch (1959); Belyaev and Levin (1972); Ogden and Birkett (1977); Mark and Vincent (1986); Okazaki et al. (1987); Aizenberg et al. $\left(2000^{a, b}\right)$; and Li and Lundgren (2002) to name only a few. There have been fewer studies conducted in calm-air chambers, including: Davies (1968); Breslin and Stein (1975); Kenny et al. (1999); and Feather and Chen (2003).

Sampling efficiency measurements conducted in wind tunnels have usually been conducted in freestream flow windspeeds from 0.5 up to around $3 \mathrm{~m} / \mathrm{s}$ (Ogden and Birkett, 1977; Mark and Vincent, 1986; Chung et al., 1987; etc.), although there have also been some studies conducted for rather high flow velocities, such as those in the range greater 
than $4 \mathrm{~m} / \mathrm{s}$ and increasing to $9 \mathrm{~m} / \mathrm{s}$ (Vincent et al., 1990). Wind tunnels used in the research have usually been large enough to contain the top half of a full-size tailor's mannequin with an appropriately small amount of flow blockage, i.e. less than $30 \%$ (See Table 1.1).

Even though there are differing results for the major work accomplished to date, the efforts made in revealing the physical characteristics of how personal air samplers behave over a range of environmental conditions has been immense. Each huge stride forward, such as the development of the IOM sampler, has been extremely important in adding more tools to the Industrial Hygienist's toolbox.

To review, large wind tunnels have shown results that can be highly variable, but are usually applicable to a wide range of workplace conditions since the experiments are of full scale (Ogden and Birkett, 1977; Mark and Vincent, 1986). Small to medium tunnels have less variability, but still require a mannequin (of some sort) to induce airflow patterns expected around a worker (Witschger et al., 1998; Aizenberg et al., 2000). However, this approach still shows differing sampling efficiency results for the various orientations to oncoming wind using differently sized torsos (Kennedy et al., 2001). Furthermore, there are turbulence effects in these conditions not fully understood that may affect the local concentration (Li et al., 2003). Finally, calm-air chambers have low variability in their results and do not need a mannequin in order to achieve useful sampling efficiency results. Calm-air chambers are applicable only to evaluations of samplers that will be used in lowairflow workplaces (Kenny et al., 1999).

All of the above air sampling evaluations were based on experiments and are thus prone 
Table 1.1: Wind tunnel and mannequin sizes for selected studies of sampling efficiency.

\begin{tabular}{|c|c|c|c|}
\hline Researchers & Wind Tunnel Dimension & Mannequin Size & $\begin{array}{c}\text { Tunnel Blockage } \\
\text { (Reported by Researchers) }\end{array}$ \\
\hline Mark \& Vincent (1986) & $2.5 \mathrm{~m}$ wide $X 1.5 \mathrm{~m}$ high & $\sim 1 \mathrm{~m}$ tall $\mathrm{X} 0.5 \mathrm{~m}$ wide & $13 \%$ \\
\hline Chung et al. (1987) & $1.22 \mathrm{~m}$ wide $X 1.83 \mathrm{~m}$ high & $1.35 \mathrm{~m}$ tall $\mathrm{X} 0.7 \mathrm{~m}$ wide & $25 \%$ \\
\hline Kenny et al. (1997) & $2.5 \mathrm{~m}$ wide $X 2.5 \mathrm{~m}$ high & $\sim 1 \mathrm{~m}$ tall $\mathrm{X} 0.5 \mathrm{~m}$ wide & $8 \%$ \\
\hline Witschger et al. (1998) & $1.83 \mathrm{~m}$ wide $X 1.22 \mathrm{~m}$ high & $0.8 \mathrm{~m}$ tall $\mathrm{X} 0.5 \mathrm{~m}$ wide & $16 \%$ \\
\hline Witschger et al. (1998) & $1.83 \mathrm{~m}$ wide $X 1.22 \mathrm{~m}$ high & $0.2 \mathrm{~m}$ tall $\mathrm{X} 0.3 \mathrm{~m}$ wide & $3 \%$ \\
\hline
\end{tabular}

to random error from any number of sources not controlled for during the investigations. Theoretical approaches to explaining the physics of air samplers, however, have received a fair amount of attention as well, and these methods -by their nature- are not prone to random error, although they are subject to other errors, such as truncation and human error. Theoretical approaches have usually been conducted with mathematical descriptions of inviscid flows near the inlets of samplers and are described in Dunnett and Ingham (1986), Dunnett (1999), and Dunnett and Vincent (2000) to name a few. The difficulty in theoretical approaches has been in calculating wide ranges of representative flow conditions. This is due to limitations in computing resources and absence of efficient numerical schemes.

Some conclusions that can be made are: (1) samplers are being used on a daily basis (to monitor exposure and for regulatory purposes) that were not designed according to uni- 
versal guidelines; (2) the universally accepted guidelines that do exist, i.e. the Inhalability convention curves, may not be applicable to some common workplace environments; and (3) prediction of sampling efficiency with computer simulation has been greatly limited due to the performance restrictions of computer hardware and available numerical schemes. 


\section{Chapter 2}

\section{Literature Review}

\subsection{Chronology of Air Sampling Efficiency Studies}

Historically, one of the earliest reports on understanding air samplers was presented by Brady and Touzalin (1911) in which they discussed the importance of maintaining sampling conditions where the sampler's inlet velocity equalled the freestream velocity, and where the sampler's inlet must be lined up with direction of freestream flow when sampling dust from blast-furnace gas. This may have been the first claim that, in order to attain a true airborne mass concentration, sampling must take place isokinetically (same speed) and isoaxially (same orientation).

Other early work includes an astute investigation by Griffiths and Jones (1939) in which they report on the evaluation of a sampling device that sampled isokinetically and isoax- 
ially. They found that if these conditions are not maintained, dynamic physics within the fluid entering the sampler's inlet would cause a bias toward under- or over-sampling of certain particle sizes. While the research in this report was conducted on an area sampler, whose performance was less than ideal, it was very important in advancing this concept in aerosol sampling.

Much later, Belyaev and Levin (1972) reported on the empirical wind tunnel investigation of particle tracks using flash illumination. Several instances of anisokinetic sampling (inlet velocity not equal to freestream velocity) were investigated as the particles entered an isoaxially-oriented sampler. The results obtained by the authors did not agree with much of the earlier work conducted. Possible reasons were given why there was poor agreement, including rebound effect from the front edge of the inlet and wall thickness on the front edge of the sampler leading to some degree of flow distortion.

The papers to follow the Belyaev and Levin limiting trajectory method began unravelling the effects of many more factors on sampler efficiency. The contributions of gravitational deposition (Heyder and Gebhart, 1977) and further work on effect of inertia (Davies and Subari, 1978) on sampling efficiency were investigated in wind tunnels. Also studied were combined factor effects including influence of Stokes' number, velocity ratio, and nozzle angle together (Durham and Lundgren, 1980). This study showed that sampling anisokinetically and anisoaxially resulted in efficiencies the Stokes' number could not predict without considering factors such as angle of orientation to freestream flow and inlet velocity.

Addlesee (1980) also addressed the topic of particle-gas interaction near the inlet of sam- 
plers. His theoretical investigation was based on a 2-D view of the air sampling problem when air and particles enter an isoaxial sampler. He used Stokes' Law $\left(S t=\rho_{p} d^{2} U_{s} \mu h\right)$, where $U_{s}$ is the inlet velocity (other variables are defined in the frontmatter), as the relationship governing the interaction between the potential flow (straightline flow, no vorticity vectors) and the particles. Addlesee computed sampling efficiencies for seven ratios of the freestream velocity to the inlet velocity against a normalized Stokes number. He found that when the ratio decreases, i.e., when the velocities get closer and closer to one another, the efficiency increases for all Stokes numbers. The contribution to the knowledge base was to demonstrate that (for 2D models) the efficiency can be reasonably predicted by theory and the numerical solution thereof.

While a number of important advances were made in the 1970s, '80s and '90s for the prediction of sampling efficiency through calculation, empirical wind tunnel experiments were continuing to expand the knowledge of air sampler physics. For example, Vincent and Gibson (1981), and Baron et al. (1994) investigated sampler efficiency values that were influenced by wall loss. Effect of turbulence on efficiency was investigated by Vincent et al. (1985), and by Weiner et al. (1988). Influence of particle bounce resulting in secondary aspiration was also investigated (Lipatov et al., 1988).

Important theoretical equations developed from experimental observations were introduced by Belyaev and Levin (1974), Grinshpun et al. (1993), and Tsai et al. (1996). Nonisoaxial sampling conditions were investigated by researchers including work by Okazaki et al. (1987) and Hangal and Willeke (1990 \& 1992). Effect of vortex formation inside 
the opening of a fiber-sampling cassette was investigated by Chen and Baron (1995), and the effect of unsteady flow at the entrance of tubular inlets was investigated by Kalatoor et al. (1995). A statistically thorough report on the testing of samplers was presented by Kennedy et al., (1995) through a NIOSH Guidance document. This document provided guidelines for how to measure the accuracy of a sampler in controlled laboratory studies in comparison to a "true" concentration.

Important work on the influence of particle behavior near the body was conducted by Rodes et al., (1995), and Sreenath et al., (1997). Several researchers developed new samplers including McFarland et al. (1989), Kalatoor et al. (1995), Torczynski and Rader (1997), Gao et al. (1997), Hauck et al. (1997), Gautam and Sreenath (1997), and Chen et al. (1998). Also, work has been done on the performance of personal samplers (Tsai and Shih, 1995), (Kenny et al. 1997), (Witschger et al. 1998), (Aitken et al. 1999), (Kenny et al. 1999), and (Aizenberg et al. 2000).

Liden et al. (2000) presented a comparison of the 37-mm cassette sampler and the IOM inhalable sampler from a workplace testing point of view rather than the traditional wind tunnel testing point of view. This study cited the following reasons that testing inhalable samplers in wind tunnels might be misleading as: (1) variable wind speeds in the workplace, (2) variable dust concentrations in the worker's area, and (3) workers influencing the source of the dust.

The difficulty of conducting air sampler research in wind tunnels has been addressed by Aizenberg et al. (2000). In this work, an attempt was made to simplify the testing 
procedures required to determine the sampling efficiency of personal inhalable samplers. It was proposed that by using a smaller wind tunnel with a simplified mannequin shape that more repeatable and more accurate results could be obtained. Aizenberg et al. (2000) claimed that results similar to those from a large wind tunnel, using a full-size mannequin, were obtained using their simplified approach. However, a report published by Kennedy, et al. (2001), contradicts the findings of Aizenberg and suggests that the size difference of a full-size mannequin and a simplified mannequin are significant and thus will lead to marked differences in sampler efficiency.

Many experiments have been conducted in wind tunnels and calm-air chambers, however, numerical and theoretical work has also been done by hand and with computers. In 1940 Vandrey described results (in Fuchs, 1975) of one of the first applications of numerical analysis ever attempted on the computational geometry of an air sampler. His calculations were done by hand with generalized boundary conditions and thus were limited in scope. However, the overall agreement with experiment was reasonable, and researchers in the field of aerosol science were able to view numerical analysis as a viable research tool.

Later, a very important numerical investigation was done by Vitols (1966) in which the limiting trajectories of monodisperse particles were determined by computational solution. An iterative method of successive over-relaxation was used to solve the laminar Laplacetype Stokes' stream function on a 2-D finite element grid. The results of the solution were used to make an approximation of the flow field entering an isoaxial sampler at nine different anisokinetic conditions. From there, slightly modified Langmuir and Blodgett dif- 
ferential particle motion equations were used to solve for particle streamlines in order to predict sampling efficiency at the various anisokinetic conditions. Vitols concluded that the numerically determined estimates of errors were in relatively good agreement with experiment. However this particular numerical investigation was conducted without inclusion of gravity or turbulence terms in the problem setup. Thus, it was not possible to conclude whether or not those terms should be included to yield more accurate results.

In the 1980s there were others who were also attempting to increase the understanding of air samplers and collection efficiency through the use of theory and numerical analysis. In 1985, Armbruster and Zebel published the results of their numerical calculations for predicting sampling efficiencies for an annular slot probe. This is a probe that has a 360degree opening (actually it is closer to 358 since four small pins hold the top on) that when looked at in cross section appears as a rectangular slot. They showed that the sampling efficiency of the annular slot sampler decreases as freestream velocity increases as well as when particle size decreases.

Since the previous two studies involved an isoaxial sampler, Liu et al. (1989) looked at the case of anisoaxial sampling. The empirical wind tunnel models developed from this investigation were somewhat more complete than those used previously because they examined the extent to which departure from traditional Stokes would cause differences in the calculations. This approach allowed them to predict the sampling efficiencies observed by the classic Belyaev and Levin (1972) experiments as well as to accurately predict sampling efficiencies for other ranges as well. While the experiment did serve to increase 
the known numerical methods for estimating sampling efficiency, it was still a simplified approach because the geometry was 2-D, there was no gravitational term, there was no effect of turbulence, and there was no measure of the contribution that thermal effects could have on particle transport. It should be noted that the methodology used in that study was consistent with those used in previous studies because the airflow was approximated as an inviscid potential flow.

Somewhat later, Chung and Dunn-Rankin (1992) deviated from methods used previously when they presented the results of their numerical studies of air flow into a 2-D sampler that included viscous terms in order to determine the effect the boundary layer had on particle transport in the near-inlet region of the sampler. Their graphical results indicated a slight, but detectable, difference between the viscous and inviscid approaches to calculate aspiration efficiency. In this work, the researchers made use of a grid-independence study to make a quantifiable choice for the cell-sizes of the computational domain, a method that became standard in the 1990s.

In that same year, Griffiths and Boysan (1992), brought finite-volume CFD software packages, FLUENT (Fluent Inc., Lebanon, NH) and CYCLOP to aerosol science in order to report on the packages' abilities to estimate the $50 \%$ cutpoint of cyclone samplers. Their report indicated that the CFD packages overestimated the 50\% cutpoint, apparently based on comparison with experimental results, in almost all configurations tested. They also concluded that the 2-dimensional models were as effective as the 3-dimensional models, although it was not clear from the paper how they determined this. 
The following year, one of the earliest inclusions of turbulence modeling in the effort to predict sampling efficiency through numerical calculation was presented. In this study, Gong et al. (1993) calculated the air flow and particle trajectories into an open tube, which they called a shroud, and then into a sampling inlet, which was "placed" just inside the downstream end of the open tube. The models were 2-dimensional and were solved with the commercial CFD code, FIDAP (finite-element solver from FLUENT, Inc., Lebanon, NH). A $\kappa-\epsilon$ two-equation turbulence model was applied to the calculation. Then the particle trajectories were calculated using Lagrangian equations. The authors concluded that the presence of the shroud caused the aspiration efficiency to increase with increasing velocity and particle size. However, they found that wall losses also increased with increasing velocity and particle size. Even though shrouds are not used on workplace samplers, this study, at the time, was the most complete CFD study of air samplers because it included wall-loss effects, buoyancy, turbulence, and Stokes drag.

Additional work was done by Yan et al., (1994) in which the efficiencies for a range of conditions around a tube sampler were calculated using an inviscid approach. The authors reported that the calculations were similar to the results of empirical wind tunnel experiments.

The airflow behavior in sampling cyclones was again investigated by Griffiths and Boysan (1996), but this time they used 3-dimensional models of the cyclones rather than the 2-D models they applied in 1992. The commercial package, FLUENT (Lebanon, NH) was again applied to the numerical calculations and particle trajectory calculations using a 
Reynolds stress model to capture the large and dominant recirculations found within cyclones. Again, as in the previous work, CFD mostly overpredicted the 50\% cutoff diameter. However, the authors were able to find agreement between simulation and experimental results for the computational geometry of a "Stairmand" type cyclone.

Until the 1990s, the numerical investigations published in the literature had largely been 2-D approximations of simplified cases of potential flow into ideal samplers. However, in the mid to later 1990 s, computers became powerful enough to solve advanced discretizations of the Navier-Stokes equations with particle trajectory calculations.

In 1999, Gao et al. published a paper in which the inlet and surface airflow behavior of a bio-aerosol sampler were calculated for a 3-D flow field in turbulent conditions. This sampler was based on the design by Liu et al. (1989), but rather than solving simplified potential flow calculations, a commercially available software package, FIDAP, was used to solve the full, 3-dimensional viscous flow field of the air and particle behavior near the inlet of the sampler.

Recently, there have been two works of note concerning numerical analysis of air samplers. The first was a paper by Dunnett and Vincent (2000) in which they presented their conclusion that neglecting the gravity term in numerical simulations of sampler efficiency would be an oversimplification resulting in increasing error as the orientation of the sampler's inlet changed from horizontal to vertical. For small particles in high-speed flows, the error is not nearly as pronounced as for larger particles in low-speed flows. The second work was a study concerning the behavior of particles in the vicinity of a wall by Tu (2000), 
which showed that wall-rebounded particles have a significant effect on the velocity of particles entering the near-wall area. This result may be important in helping to quantify the loss of particles to the wall in the inlet of a personal sampler.

Some of the most diligent work to date on the simulation of air samplers and personal exposures has been performed by Flynn et al. with several related papers published in 1996, 1999, 2000, 2001, and 2002 (Tan, et al.). In one report, (Flynn and Sills, 2001) an effort was made to use the commercial CFD software, FIDAP, to predict the airflow behavior and particle trajectories for two particle sizes around a cylinder used to approximate a human torso.

Additional work has been done by Soon Lan and Viswanathan (2001) with an investigation of the effect of a simplified human-like bluff body on the airflow into a fume hood using FLUENT software (Fluent, Inc., Lebanon, NH). Their most significant finding was that 2-D simulations of this geometry did not agree with the 3-D models and so they concluded that only 3-D models should be used.

Recently, work has been presented by Lo Savio et al. (2003), describing scaling experiments for determination of aerosol sampler efficiency. Scaling experiments have been discussed in the aerosol science arena for some time, but the attempts at doing them have been limited. In this study the authors reported that their scaling efforts resulted in values that agreed with existing data. However, the numerical results still remain to be validated. Also, the sampling efficiency calculations were conducted on an air sampler that was free in the air rather than being mounted on a bluff body, which has typically been the preferred 
method used in wind tunnels.

Currently, there is ongoing work by a group at West Virginia University where attempts are being made to calculate the airflow behavior around full-size human-like torsos ( $\mathrm{Li}$ et al., 2003). The turbulence in the near-body region is being investigated along with the degree of detail a model requires for approximating airflow around a human-like torso.

\subsubsection{Summary}

The history of experimentation and simulation in the field of occupational safety and health has had a long and evolving impact on how samplers are used in the workplace. Application of advanced analysis tools, such as CFD, to the field of aerosol exposure monitoring will hopefully continue to yield greater understanding of personal aerosol samplers in order that workplaces with aerosol overexposures are correctly evaluated and better controlled.

\subsection{Development and Application of Theories in Aerosol Sampling}

This section is broken into three primary subsections: The Inhalability curve, Determination of the "True" Aerosol Concentration, and Application of Numerical Methods to Simulation of Aerosol Samplers. 


\subsubsection{Aerosol Aspiration in Human Models: Inhalability Convention}

Air samplers are used to gain an understanding of the concentration, size, and type of particle exposure present in workers' environmental atmospheres. Therefore, a discussion of air sampling devices is incomplete without an examination of the aspiration of aerosols into the human airway.

As described by Georgius Agricola in 1556, and later by Bernardino Ramazzini in 1700 (Rose, 1997), exposure to dust and smoke has been a problem for humans for hundreds of years. However, it wasn't until the industrial revolution in the mid to late 1800 s that both the problem and attempts at solving it began to accelerate (McClellan, 2000). In the early 1900s, post-mortem excisions of worker's lungs for various industries revealed differing lung responses for the differing exposure environments (Mauderly et al., 2000). Furthermore, there was clearly a relationship between the size of the particles found in the lung and depth of penetration therein.

An important problem in human exposure evaluation has been determining the inhalability of various sizes of particles to different regions of the airway after aspiration. A number of studies were conducted to investigate these parameters. However, in earlier years researchers decided that penetration of a particle of any size may cause damage. Therefore the protective approach would be to simply categorize aspiration as penetration of particles past the nose and mouth rather than deposition.

This was accomplished through a fairly substantial amount of research using breathing 
mannequins. Experiments were conducted to estimate the inhalability of a human and develop an equation relating aspiration efficiency to particle size. As a result, Ogden and Birkett (1977, 1978), Armbruster and Breuer (1982), and Mark and Vincent (1986) identified a common inhalability curve as,

$$
S I(d)=0.5\left(1+e^{-0.06 d}\right)
$$

where $S I$ is sampling efficiency for the inhalable fraction and $d$ is the aerodynamic diameter of the particle.

This curve has a collection efficiency of $100 \%$ for small particles, and decreases to $50 \%$ collection efficiency for particle sizes larger than $40 \mu \mathrm{m}$. The curve is valid from 1 to 100 $\mu m$ based on "convention." Beyond $100 \mu m$ there may still be health effects from inhalation of large particles, but there is not a general agreement for what their collection efficiency should be.

The Inhalability convention is intended to be a performance curve for evaluation and for comparison of mannequin-mouned aerosol samplers collecting particles from 1 to $100 \mu \mathrm{m}$ in a wind-tunnel setting that uses directional averaging over 360 degrees for wind speeds from 0.5 to $8 \mathrm{~m} / \mathrm{s}$. For windspeeds less than $0.5 \mathrm{~m} / \mathrm{s}$, it likely does not apply since no data have been collected for the Inhalability convention below this windspeed. Rather, this is the realm of calm-air or low-velocity sampling, which has been shown by Kenny et al. (1999) and Feather and Chen (2003) to yield drastically different sampling efficiencies in 
aerosol samplers than does the Inhalability curve for its applicable range. Despite the lack of convention for calm-air sampling, Kenny et al. (1999) suggested that the low-airflow testing regime yields more repeatable results using simpler experimental setups. It is not unreasonable to expect that future experiments will establish a performance curve similar to what Kenny et al. (1999) have reported.

Both wind-tunnel and calm-air experiments require a "grounding" or reference point of comparison. This has usually been an isokinetic, isoaxial thin-walled sampling inlet that is intended to collect the "true" concentration in air. For the determination of inhalability, these reference samplers were used to measure the baseline concentration, and then the breathing mannequins were used to determine an inhalability curve.

In calm-air, the determination of a reference concentration has proven much more difficult. Aitken et al. (1999) found agreement between the horizontal, vertical, and circling probes, and best repeatability for circling probes, and thereby they adopted this method to determine the reference concentration. They found disagreement between circling probes and the gravimetric method as well as between stationary probes and the gravimetric method. This finding was confirmed by results collected by Feather and Chen (2003).

Thus, in experimental studies it has been shown that there is clearly a disconnect between moving- and calm-air sampling, a result which is likely translated to workplaces. However, the same reference sampler method may be used in both regimes. Even so, further research is needed to establish if use of samplers evaluated against the Inhalability curve for deter- 
mining airborne exposure is protective in calm-air workplaces. The results from Kenny et al. (1999) suggests this to be the case. However, it does need to be confirmed with additional studies.

\subsubsection{Theoretical and Experimental Methods for Determining the Ref- erence or "True" Concentration - The Stokes Number Approach}

For nearly 100 years, it has been the goal of researchers and practitioners to assess airborne dust concentrations. Early on there was a major emphasis on determining a truly representative concentration. The use of thin-walled sampling probes collecting particles from undisturbed air was believed to be the best method for this assessment (Brady and Touzalin, 1911). However, it proved to be extremely difficult to achieve this condition, and thus researchers began to explore both ideal sampling conditions and less than ideal conditions.

As a prelude, it is important to present Stokes' law for particle motion in fluids. Particle Stoke's number is defined as the relationship of the stopping distance of a particle to its characteristic dimension and is calculated as follows:

$$
S t k_{p}=\frac{\tau U_{0}}{d_{c}}
$$

where $\tau$ is the particle residence time determined from $\tau=\frac{\rho_{0} d_{a}^{2} C_{c}}{18 \eta}, U_{0}$ is the freestream velocity, and $d_{c}$ is the characteristic dimension of the obstacle that the particle encounters. The Stokes number is often referred to as $K$, as is the case in the papers described in the 
following paragraphs. The additional terms are: $\rho_{0}$ - particle density; $d_{a}-$ aerodynamic diameter of particle; and $C_{c}-$ Cunningham slip correction factor, which is a function of the particle diameter and mean free path between particles. For the physical parameters considered in the above relation, particle Stoke's numbers greater than 1 will continue in a straightline distance when the fluid (airflow in this case) changes directions. For particle Stoke's numbers smaller than 1, the particle will follow the streamlines of the airflow (Hinds, 1999).

Among the important early attempts to understand the physics of inlet aspiration was the work by Davies (1947) in a calm-air environment in which he showed a relationship of unity between the concentrations collected by samplers and the true concentration. This result holds true in recent work presented by Kenny et al. (1999) and Feather and Chen (2003). In efforts to understand anisokinetic sampling, Watson (1954) and Badzioch $(1959,1960)$ discussed theoretical approaches to the problem and described results of their experiments.

Watson's approach was to present a relation for sampling efficiency as follows,

$$
\frac{C}{C_{0}}=K+\frac{U_{0}}{\bar{U}}=\frac{U_{0}}{\bar{U}}\left[1+f(p)\left(\left(\frac{\bar{U}}{U_{0}}\right)^{1 / 2}-1\right)^{2}\right]
$$

where, $\frac{C}{C_{0}}$ is the ratio of inlet concentration to true concentration; $K$ is a function of particle size and is proportional to a disk-shaped area upstream from the inlet, across which a particle must travel to be aspirated; $\frac{U_{0}}{U}$ is the ratio of freestream velocity to inlet velocity; and $f(p)$ is a function of the particle's inertial properties as it approaches the inlet. According to Watson, the crux of the problem was determining the motion of the particle across $S$, 
which he considered to be the diameter of a surface located some distance upstream from the inlet. If the particle was to be aspirated, it had to travel into the region of $D$, the inlet diameter, which was either larger or smaller than $S$, depending on whether freestream was greater or less than inlet velocity. He sought to determine the particles' inertial influence experimentally, thus validating the approach for the conditions he proposed.

Watson (1954) conducted experiments in a wind tunnel using lycoperdon and lycopodium spores of 4 and $32 \mu \mathrm{m}$ diameter, respectively. This was done to determine the unknown components of the above relation, namely $f(p)$ in order to derive $K$. Watson used three different diameters $(0.46,0.7,1.05 \mathrm{~cm})$ of 90 -degree glass inlets leading to an impinger to sample the spores from constant velocity freestream airflow. Since freestream was constant but inlet velocity was varied, $\frac{U_{0}}{U}$ was subsequently varied. The $0.7 \mathrm{~cm}$ diameter inlet was chosen as the reference, although the reason for this decision was not revealed. The results obtained from this experiment were reported by the author to be suspect due to the difficulty in determining the exact spore diameters. Even so, the results were consistent enough to reveal the trend that, for the particles used, sampling efficiency increased as $\frac{U_{0}}{U}$ increased. However, Watson was unable to confirm the relation he presented. In the same paper, Watson presented some interesting results from work conducted by his colleagues, J.E. Mayhood and G.O. Langstroth, for analysis of anisoaxial sampling conditions. Their work confirmed that increasing angle from isoaxial sampling resulted in reduced sampling efficiency for the three particles sizes they tested $(3,12,37 \mu \mathrm{m})$.

Following Watson by several years, Badzioch (1959) also investigated the effect of vary- 
ing $\frac{U_{0}}{U}$, which he referred to as $\frac{V}{V_{S}}$, on the sampling efficiency, $\alpha_{0}$ (i.e. $\frac{C}{C_{0}}$ from Watson). Badzioch made the statement that the collection efficiency, $\alpha_{0}$, was a function of the Stokes dimensionless parameter, $K$ (slightly different from Watson's Stokes $K$ ), which was given by,

$$
K=\frac{(\sigma-\rho) d^{2} V}{18 \eta D}
$$

where $\sigma$ is the density of the particle, $\rho$ is the density of the fluid, $d$ is the diameter of the particle, $V$ is the freestream velocity, $\eta$ is the viscosity of the fluid, and $D$ is the diameter of the sphere or obstacle performing the sampling. Badzioch followed a more traditional Stokes law treatment than did Watson and presented the following relation for determining collection efficiency,

$$
\alpha=\frac{1-\exp (-L / \lambda)}{L / \lambda}
$$

and for consideration of impaction effects,

$$
\alpha_{0}=\frac{1-\exp (-n / K)}{n / k}
$$

where $L$ is the length of upstream disturbance due to the sampler, $K=\lambda / D$, and $\lambda$ is the stopping distance of the particle. To confirm the relation, experiments were conducted in a recirculating "wind tunnel" made from steel pipe eight inches in diameter. Polydisperse 
dusts of Aerocat (an amorphous silica-aluminum amalgam) and zinc spheres were 19, 23, and $27 \mu \mathrm{m}$ as Badzioch determined from Fairs' microscope method. Five different inlets, ranging in size from 0.653 inch up to 1.896 inches were exposed to the dust in the tunnel, which was collected on glass fiber filters in the samplers. Dust was recirculated in the tunnel continuously through the measurements. Badzioch noted that there was a fall in dust concentration over time and to account for it experimentally, he alternated isokinetic and anisokinetic samples. He also reported that there was not a detectable change in collection efficiency with change in nozzle size and that this was likely due to the wide spread of the data. Badzioch concluded the paper with a graph that expressed increasing particle collection efficiency with increasing stopping distance, which he recommends be determined from one of the following:

$$
\begin{array}{r}
\lambda=\frac{(\sigma-\rho) d^{2} V}{18 \eta} \\
\lambda=\frac{v V}{g}
\end{array}
$$

where $v$ is the free-falling velocity of the particle.

One year later, Badzioch (1960) appended his previous work by presenting equations for determining concentrations for fine and coarse dusts. Both are simple variants of the traditional mass per unit volume method. In addition, corrections were proposed for anisokinetic sampling based on plotting the data and finding the point where $\frac{V}{V_{s}}=1$, which Badzioch claimed was where the true concentration would be found. 
Other research followed Badzioch, but it was Belyaev and Levin (1972) who set out to truly quantify aspiration efficiency of thin-walled probes. They summed the problems of previous experimenters into three categories: not considering deposition in the inlet, rebound from the outside of the sampler's inlet, and poor control on tube diameter. From this, they presented a functional approximation for "aspiration efficiency" (referred to as “collection efficiency" by Badzioch, and "sampling efficiency" by Watson) as follows,

$$
A=A_{d} \cdot A_{y} \cdot A_{i}
$$

where $A_{d}$ is the aspiration component due to deposition, $A_{y}$ is the aspiration component due to particle rebound, and $A_{i}$ is the aspiration component due to aerodynamic and inertial forces.

Belyaev and Levin focused their experimental efforts on $A_{i}$, which they considered to be a function of $\frac{\bar{u}}{u_{0}}, K$, and $\frac{D}{d}$, where $\bar{u}$ is the mean inlet velocity, $u_{0}$ is the undisturbed freestream velocity, $D$ is the outer diameter of the tube sampler, and $d$ is the inner diameter of the tube sampler. In an effort to greatly simplify the physical conditions, they investigated one orientation of the sampling probe (isoaxial), used a monodisperse aerosol (willow pollen and Lycopodium spores), used five different sampling probes for their tests, and nine different Stokes numbers. Flash photographs were taken of the aerosol particles in the wind tunnel and their tracks were captured. These data were used to determine the points of the "limiting" trajectories relative to each sampler and wind tunnel condition tested. 
In their paper, Belyaev and Levin described the potential source of errors, and focused on $\phi$, diameter of an imaginary "disk" of particles located at some distance upstream from the inlet (like Watson's "S") that will subsequently be sampled, as a possibly large unknown. From the experiments, the researchers found the following: lower aspiration efficiencies at specified $K$ than previous investigators observed; the value of $A_{i}$ approaches $\frac{u_{o}}{\bar{u}}$; disagreement existed with Levin's theory that $A_{i} \rightarrow 1$ as $\frac{u_{0}}{\bar{u}} \rightarrow \infty$; and that $A_{i}$ values near unity were obtained using thin-walled probes sampling at nearly isokinetic conditions. Finally, the authors investigated reasons why their results differed from others and proposed particle rebound at the inlet as a likely possibility.

Historically, the Belyaev and Levin paper was critical for a number of reasons. First, they demonstrated that wall thickness was an extremely important factor contributing to the concentration "seen" by the sampler. Their experimental findings confirmed that the use of sharp-edged, thin-walled inlets had little disturbance on the flow field, and that this would be the preferred sampler inlet design for determining true concentration. Previous to their findings, dull-edged probes had been used quite commonly. Secondly, their results showed the importance of using monodisperse particles, which they referred to as "isodisperse", in order to effectively vary Stokes number based on changes in freestream velocity. Finally, they presented a sound argument for the existence of a "limiting trajectory" approaching a sampler that, based primarily on $\frac{\bar{u}}{u_{0}}$ and particle size, will define the concentration collected by the sampler. Their results effectively confirmed the importance of isoaxial and isokinetic sampling with thin-walled probes as a means for determining the true concentration, and 
that the total efficiency, $A_{e}$ was dependent on more than just the inertial parameters of a particle.

Soon after their 1972 paper, Belyaev and Levin published another article (1974) further clarifying methods for determining true aerosol concentrations. In that paper, they focused on identifying the sources of errors influencing the determination of true concentration. They presented the following possible sources of errors as wall thickness, inexact $\bar{u}$ and $u_{0}$, and flow velocity fluctuations. For a general relation to predict sampling efficiency, they presented:

$$
A_{i}=1-3.2 \sqrt{\frac{u_{0}}{u}} K+0.44 \frac{u_{0}}{u} K^{2}
$$

The above is intended for isoaxial sampling and nearly isokinetic sampling. For conditions outside this range, they suggested identifying from Stokes $K<0.5$ and $K>0.5$, such that for the former, $U$ in the following equation (determination of $C_{0}$ ) is mean inlet velocity, while for the latter, $U$ is the mean freestream velocity. Concentration is

$$
C_{0}=\frac{4 N}{\pi d^{2} U t}
$$

where $C_{0}$ is the concentration, $\mathrm{N}$ is the mass of the particles registered during period of sampling time, and $t\left(\pi d^{2} / 4\right) U$ is the volume flowrate into the sampler. This method is applicable for $u_{0} / \bar{u}>0.2$. 
The efforts leading up to and including Belyaev and Levin's 1974 paper were all very important because they helped to build a sound theoretical and experimental base for understanding the behavior of aerosol samplers. Gibson and Ogden (1977) continued the work with studies of sharp-edged probes in calm-air conditions. They argued that the relations obtained from the previous work were not applicable to calm-air conditions, the reason being that "calm-air results imply a change at the lower wind velocities." For their experiment, Gibson and Ogden used fluorescent tagged Uvitex-OB spheres in a calm-air chamber having flow velocity on the order of $0.05 \mathrm{~m} / \mathrm{s}$. Three different probe diameters were used in the chamber: 3,5 , and $10 \mathrm{~mm}$. The concentrations determined from horizontally and vertically placed probes were very similar for all three probe diameters and for 6 different inlet flowrates. Their results showed that the probe diameter, inlet flowrate, and orientation were not critical for collection of an accurate measure of the concentration. Gibson and Ogden then placed their 5-mm sharp-edged probe into a wind tunnel and challenged it with 9 and $19.5 \mu \mathrm{m}$ particles in windspeeds of 0.4 to $4.0 \mathrm{~m} / \mathrm{s}$. From the wind tunnel measurements, they observed results similar to those in previously conducted studies, such as Belyaev and Levin $(1972,1974)$. Their work reinforced the need to used sharp-edged, thin-walled probes for accurate sampling in flowing air environments. In addition their results showed that a representative sample in a calm-air environment could be obtained from a horizontal or vertical probe.

At nearly the same time as the Gibson and Ogden paper, Davies and Subari (1978) presented results of their work on thin-walled sampling tubes sampling at a 90-degree "yaw" 
to the direction of freestream flow in very low airflow conditions. They used the results of their experiments, together with existing empirical relations for calculating aspiration efficiency, and extended the known equations to include their yaw angles. In particular they used variations of the Stokes number for inlet and freestream conditions to make, what they considered, improvements on the relation from Levin (1957). The authors felt there was a significant improvement on capturing a perceived inertial lag at the entrance to the sampler.

Durham and Lundgren (1980) followed Davies and Subari's work, but rather than focusing on calm-air, presented results conducted for sampling efficiency of sharp-edged probes in a wind tunnel where Stokes number was varied from 0.007 to 3.0. Their results showed decreasing sampling efficiency with increasing Stokes number and angle of orientation. The experimental values agreed with the calculated values, and a predictive equation was presented for aspiration that took into account the Stokes number, a modified Stokes number, angle of inlet orientation with respect to freestream flow, and velocity ratio. The equation is as follows

$$
A=1+(R \cos \theta-1) \frac{\beta\left(S t^{\prime}, R\right)}{\beta\left(S t^{\prime}, R=1\right)} \beta^{\prime}\left(S t^{\prime}, \theta\right)
$$

where $A$ is the aspiration efficiency; $R$ is the ratio of freestream velocity to inlet velocity; $\cos \theta$ is the angle of the inlet with respect to freestream velocity direction, $\beta(S t)=[1-$ $\exp (-L / l] /(L / l)$, with $L$ is the distance upstream that presence of the sampler has an influence and $l$ is the particle stopping distance; $S t^{\prime}=S t * \exp (0.022 \theta)$; and $\beta(S t, R)=$ $1-\frac{1}{1+(2+0.617 / R) S t}$. 
This equation was modified slightly by Vincent et al. (1986) with the addition of a $k$ empirical coefficient and consideration of particle relaxation time in the inlet of the sampler with the resulting equation as follows

$$
A_{t}=1+\left[1-\frac{1}{1+k S t\left(\cos \theta+4 R^{1 / 2} \sin ^{1 / 2} \theta\right)}\right] *[R \cos \theta-1]
$$

where $A_{t}$ is the "theoretical aspiration", $k$ is the empirical coefficient, $S t$ is the Stokes number $\left(S t=d_{a e}^{2} \gamma^{*} U / 18 \eta D\right), \theta$ is inlet angle with respect to freestream flow, and $R$ is the ratio of freestream to inlet velocity. Vincent compared this relation with the results from Durham and Lundgren (1980) and with Davies and Subari (1982) finding that their measurements were quite similar. The data from Vincent et al. (1986) had larger scatter, which was attributed to using a big wind tunnel. Vincent (1987) followed up this work with a discussion of ideal values of the empirical coefficient $k$ and also with suggestions for inclusion of a "bluntness", $B$, in order to expand the above equation to larger sized samplers. Shortly after, based on the previous work in calm and moving air, Grinshpun et al. (1993) presented two equations to further define aspiration efficiency. The first was for calm air and is

$$
\left(E_{a}\right)_{c a l m, \phi}=\exp \left(-\frac{4 S t k_{i}^{\left(\frac{V_{s}}{U_{i}}\right)^{\frac{1}{2}}+1}}{1+2 S t k_{i}}\right)+\frac{V_{s}}{U_{i}} \cos \phi
$$

for $\phi=0-90^{\circ}$. The second was for moving air and is 


$$
E_{a} \approx\left(E_{a}\right)_{m o v, \theta}(1+\delta)^{\frac{1}{2}} f_{m o v}+\left(E_{a}\right)_{c a l m, \phi} f_{c a l m}
$$

for $\theta=0-90^{\circ}, \phi=0-90^{\circ}$, where

$$
f_{\text {mov }}=\exp \left(-\frac{V_{s}}{U_{w}}\right), f_{\text {calm }}=1-\exp \left(\frac{V_{s}}{U_{w}}\right)
$$

Grinshpun et al. (1993) reported that agreement between the above theoretical relation for moving air and experiment was very good, but only a few data sets were compared. For calm-air, detailed comparison with existing data was not done, thus it is not known how well the relation compares with experimental results.

Grinshpun's work, and all the efforts previous to his, have been very important in pushing forward the understanding of aerosol sampling. All the work to date has been based on relating aspiration efficiency to the Stokes number. This was done because, as Vincent (1987) has said, “...numerical solutions for all except the simplest systems require large computing resources, and, for the most (more complicated) practical devices, it is not at present a realistic option. Most researchers have recognized this and therefore adopted a more pragmatic approach based on knowledge of the broad features - rather than a rigorous description - of the air flow." 


\subsubsection{Numerical Methods for Determining the Reference Concentra- tion}

Deterministic approaches to calculate the aspiration efficiency of aerosol samplers have been ongoing since at least the mid 1900s. However, lately, with the advances in theory and computing power in the latter half of that century, complex numerical studies were made possible.

Of these studies, Addlesee (1980) made use of Stokes' law as the major factor governing aerosol interaction with airflow in an Eulerian reference frame. In that work, a potential flow approach was used to approximate the airflow behavior entering the inlet of a thinwalled aerosol sampler. This was accomplished by considering the flow rate of the fluid entering the sampler and bounding the region outside the sampler based on the "circle theorem," for which Addlesee cited Milne-Thomson (in Vol. 36, Proceedings of the Cambridge Philosophical Society, 1940). The potential flow scenario was created when singularities from within the sampler region and outside of that region are summed, such that source and sink terms are effectively captured. In this case, vorticity of the fluid is neglected. Addlesee then applied the streamline method to the potential situation and presented an integral relation that could be solved numerically as,

$$
\sigma_{2}=\sigma_{1}+\int_{\zeta_{1}}^{\zeta_{2}} \frac{d \zeta}{\left(\frac{d \zeta}{d \sigma}\right)}
$$

where $\sigma_{2}$ is new velocity at position $\zeta_{2}$ and $\sigma_{1}$ is known velocity at position $\zeta_{1}$. Based on 
the velocities, the author calculated particle motion from

$$
\ddot{\zeta}=\frac{18}{\pi S t}(\hat{\sigma}-\dot{\zeta})+\frac{\rho}{\rho_{p}} \sigma \frac{\hat{d} \sigma}{d \dot{\zeta}}
$$

where $\ddot{\zeta}$ is particle acceleration, $S t$ is the Stokes number, $\hat{\sigma}$ is the complex conjugate of the velocity, $\dot{\zeta}$ is the first derivative of position, $\rho$ is the density of the fluid, $\rho_{p}$ is the density of the aerosol, and $\sigma$ is the velocity calculated from the potential flow equation. Addlesee used these equations (2.17 and 2.18) to calculate collection efficiency, $\eta=l / h$, where $l$ is width of undisturbed flow, and $h$ is width of sampler. He assumed that small particles would be well within the flow streamlines, but for larger particles found that inertial characteristics began to dominate. Addlesee further defined the particle trajectory range with the argument that $l$ was the "upstream asymptote" and as such an aerosol would have to follow a limiting trajectory. This led to a maximum collection efficiency, $\eta_{\max }$, which does not exceed $1-$ $\frac{3(r-1)}{2 r} \sqrt{\frac{h}{\pi x_{0}}}$. From the calculations, Addlesee found that, generally, the efficiency increased as Stokes number increased for the ranges he tested, which were $r$ values greater than 1 . This result is consistent with other findings. Finally, he indicated that for large Reynolds number situations where Stokes law may not apply, the particle drag will depend on fluid viscosity $\mu$ and fluid density $\rho$.

Following Addlesee, Ingham (1981) presented his calculations on the sampling efficiency of a "blunt" sampling head. Like Addlesee, he also used a potential flow treatment to calculate airflow and applied Stokes law to capture particle motion. The geometry used was a cylindrical shape and Ingham focused on calculating $s / a$, which is the ratio of $1 / 2$ width 
of limiting trajectory on the body to the sampler's characteristic dimension. In addition, the bluntness, $B$ was also determined for the geometries and conditions tested.

Several years later, Dunnett and Ingham (1986) presented calculations from application of their Linear Boundary Integral Equation (L.B.I.E.). This is a method based on an inviscid perspective of the airflow behavior. Using the L.B.I.E., the authors were able to calculate the flow field up to two diameters from the surface of a cylinder (Ingham, 1981).

These works previously conducted consisted of inviscid, irrotational (potential) flow near the inlet of the respective sampler. Chung and Dunn-Rankin (1992) reported on a numerical study to determine the aspiration efficiency based on a viscous fluid approach by applying discretizations of the continuity and 2-D momentum equations, which are

$$
\begin{array}{r}
\frac{\partial u}{\partial x}+\frac{\partial v}{\partial y}=0 \\
\rho\left(u \frac{\partial u}{\partial x}+v \frac{\partial u}{\partial y}\right)=-\frac{\partial p}{\partial x}+\mu\left(\frac{\partial^{2} u}{\partial x^{2}}+\frac{\partial^{2} u}{\partial y^{2}}\right) \\
\rho\left(u \frac{\partial v}{\partial x}+v \frac{\partial v}{\partial y}\right)=-\frac{\partial p}{\partial y}+\mu\left(\frac{\partial^{2} v}{\partial x^{2}}+\frac{\partial^{2} v}{\partial y^{2}}\right)
\end{array}
$$

Chung and Dunn-Rankin used Patankar's (1980) formulation to develop the discretizations, which consists of use of the SIMPLE algorithm (Semi-Implicit Method for Pressure Linked Equation) (Patankar, 1980). Particle motion was approximated from derivations of Newton's second law (the authors used the method from Vitols, 1966) and then Stokes law was applied. Several calculations were conducted to determine the sensitivity of the results 
to computational grid, and a medium density grid (121 X 86 grid lines) was chosen based on their grid convergence analysis. Velocity vectors were then calculated around a blunt cylindrical sampler to determine the diverging streamlines and particle trajectories were calculated. The authors found that their results were similar to experiments and inviscid calculations previously conducted with the exception of what they called the "overshoot," "decline," and "dip" regions where the fluid velocity field and the particle inertial force competed for control of the particle trajectory. Their results indicated there were some differences between viscous and inviscid calculations, although whether the degree of the difference has a major impact on results is not known.

\subsubsection{Summary}

The work conducted to date on aerosol samplers establishes that there is a predictable size range of particles that penetrate into the human airways. Determining the concentration of those particles can be accomplished with both empirical and theoretical models that can be compared to concentrations seen by "real-world" aerosol samplers. Finally, numerical methods can be used to predict sampling efficiencies for ranges beyond those looked at with wind-tunnel and workplace experiments. 


\subsection{Background for Use of CFD in Calculating Efficiency of Aerosol Samplers}

\subsubsection{Determination of Airflow Velocity}

The Navier-Stokes equations are the governing equations of fluid flow. These equations were derived independently by the gentlemen for whom the equations are named. According to historical accounts, Navier first derived in the equations in 1827, but it was Stokes who was able to place them in the context of a fluid property, specifically the viscosity (White, 1974). To this day, the approaches of "viscous" versus "non-viscous" (or inviscid) are used to describe a particular perspective of how to treat the prediction of fluid behavior. For very simple flows, where separation of the fluid does not occur and for which there is no turbulence, the inviscid approach may be applied. Mathematical treatment and exact solutions for inviscid flows are readily doable. However, these flows are few in number because in almost every circumstance of "real-world" flow there is separation, fluctuation, and turbulence. To account for these features, it is necessary to apply discretized forms of the Navier-Stokes equations in numerical schemes in order to capture them. The Navier-Stokes equations are nonlinear, nonunique, and have few exact solutions. Thus it is necessary to simplify them into more readily usable forms. These are discussed in detail in Chapter 3, Research Methods.

A number of studies have been conducted on aerosol samplers in which numerical solutions of the Navier-Stokes equations were applied in order to calculate the viscous flow 
features. Some of these studies include Griffiths and Boysan (1996), where the CFD code FLUENT was used to simulate flow in cyclone samplers; Gao et al. (1999), where the CFD code FIDAP (Fluent, Inc., Evanston, IL) was used to calculate airflow entering a bio-aerosol sampler; and Lo Savio et al. (2003), who used FLUENT to simulate airflow entering and passing around samplers in freestream air.

\subsubsection{Calculation of Particle Trajectories in a Lagrangian Reference Frame}

Computationally, reference concentration can be determined in exactly the same way as it is done theoretically. For instance, Vincent et al. (1986) define theoretical aspiration efficiency as, $A_{t}=N / N_{0}$. In this relation, $N$ is the number of particles per unit time entering the sampler's inlet and $N_{0}$ is the number of particles passing a point in space per unit time not subject to flow distortions due to bluff objects, such as a sampler.

Particle motion through a fluid can be "viewed" from one of two perspectives: Eulerian or Lagrangian. The Eulerian approach considers the motion of aerosol from the perspective of the fluid it moves through, such as watching from the sidelines and tracking the particle through the domain. The Lagrangian approach considers particle motion from the perspective of riding the particle through the fluid. Eulerian approaches are often used for mixes of fluid and aerosol where the aerosol concentration is significant in relation to the fluid in which it travels, such as mud (clay) in a volcanic lahar flow. The Lagrangian approach is used when the aerosol concentration is much lower than the surrounding fluid, such as dust 
in workplace air.

Gao et al. (1999 and 2002) used the Lagrangian formulation to simulate particle trajectories in a bio-aerosol sampler and the Button inhalable sampler. Results of the bio-aerosol simulation agreed well with experimental values from Gao et al. (1997) and Armbruster and Zebel (1985).

\section{Turbulence Considerations}

Turbulence has been shown to exist in wind tunnels where aerosol sampler research has been done (Ogden and Birkett, 1978). Since eliminating turbulence is too difficult, determining its contribution, if any, to sampling efficiency was the approach used by Vincent $e t$ al. (1985). Vincent and colleagues found that low levels of turbulence intensity $(<10 \%)$ do not appreciably contribute to the sampling efficiency of thin-walled probes. However, for blunt samplers the contribution of turbulence was much more difficult to ascertain.

In either case, it is important to control the turbulence so that its effect is minimized. Vincent et al. carried out their work in a large wind tunnel $1.5 \mathrm{~m}$ high X $2.5 \mathrm{~m}$ wide $\mathrm{X}$ $4 \mathrm{~m}$ long. Thus, there was likely the presence of relatively large-scale turbulence. The authors reported turbulence intensities ranging from $3.5 \%$ up to over $15 \%$. Length scales were reported from 0.02 up to $0.1 \mathrm{~m}$.

These two values, turbulence intensity and length scale, give much information about the turbulence present in a dynamic fluid environment. From them, estimates of the turbulence 
production and expected dissipation can be generated. For the work in this dissertation, the results from Vincent suggest that turbulence intensity is not likely to have a significant effect on sampling efficiency. The length scale on the other hand is very important because it can not be accurately captured without the proper grid sizing, which has density that can not be correctly defined without an estimate of the size of the minimum turbulence length scales. These typically occur at the surface of a bluff body and at the shear between slow and fast moving fluids.

A starting point for determining boundary and shear layer thicknesses may be obtained through consideration of several important concepts in turbulence. The first concept concerns the cascade of energy from large scales of turbulence motion (large eddies) to smaller and smaller eddies until the energy is dissipated at very small scales as heat energy through viscous friction (Richardson, 1911).

This concept was followed by an expression from Taylor (1935) where the production of the energy was related to the integral of the wavenumber (reciprocal of wavelength) according to the following relation

$$
k=\int_{0}^{\infty} E(\kappa) d \kappa
$$

where $k$ is the kinetic energy per unit mass and $E(\kappa)$ is the dependence of the turbulence kinetic energy on the distance between wavenumbers.

Since the focus of the Richardson concept is on energy dissipation and $k$ is concerning 
production, a relation must be made to account for the loss of energy through the turbulence and viscous action. Taylor accomplished this with dimensional analysis and presented the following relation

$$
\epsilon \sim \frac{k^{3 / 2}}{l} \Longrightarrow k \sim(\epsilon l)^{2 / 3}
$$

where $\epsilon$ is the energy dissipation, which is dependent on the length scale $(l)$ and the turbulence kinetic energy $(k)$.

The final important concept is by Kolmogorov (presented in Tennekes and Lumley, 1972 and also in Pope, 2000). This concept is founded upon the assumption that the smallscale motions of turbulence are statistically independent from the large-scale motions and are dependent only upon the rate of energy supply arriving from the next highest scale of motion. Thus, it is further assumed that the energy supply and dissipation are in fact equal.

From there, Kolmogorov surmised that the most important factors contributing to the energy -which was causing the small-scale turbulence motions- were limited to only two sources: the energy supply from the next highest scale (dissipation), and the viscosity (kinematic) of the fluid. Using this concept as a basis, the following Kolmogorov microscales were presented

$$
\eta \equiv\left(\nu^{3} / \epsilon\right)^{1 / 4}
$$




$$
\begin{gathered}
\tau \equiv(\nu / \epsilon)^{1 / 2} \\
v \equiv(\nu \epsilon)^{1 / 4}
\end{gathered}
$$

where $\eta$ is the length scale, $\tau$ is the time scale, and $v$ is the velocity scale.

Returning to Taylor (1935) and using the assumption that supply rate is equal to the dissipation rate, the large scale motions, which are usually dominated by inertial effects, can become the basis for a relation describing the dissipation. This is represented as follows

$$
\epsilon \sim \frac{u^{3}}{l}
$$

where the $u$ and $l$ are velocity and length at the largest scales of motion. This relation makes the assertion that the large eddies are the dominant energy "movers" in the energy cascade. Tennekes and Lumley (1972) presented scaling relations for length, time, and velocity for the Kolmogorov microscales, in functions of the large-scale Reynolds number as follows,

$$
\begin{aligned}
& \frac{\eta}{l} \sim R e^{-3 / 4} \\
& \frac{\tau}{T} \sim R e^{-1 / 2} \\
& \frac{\nu}{v} \sim R e^{-1 / 4} .
\end{aligned}
$$

Using the above, estimates can be made of turbulence length scales. Applying $R e^{-3 / 4}$ for the airflow in the wind tunnel around a box-shaped blunt body (suppose freestream = 
$0.5 \mathrm{~m} / \mathrm{s}$, cross section $=1.5 \mathrm{~m})$. Then a minimum Kolmogorov length scale can be estimated as approximately 0.005 . Using this value as the minimum scale and 0.01 as the maximum (same order of magnitude measured by Vincent et al., 1985), a starting range for the expected size of the turbulence length scales results. In order to best capture the turbulence effects in this size range using CFD simulations, parametric studies are performed by applying a well-established two-equation turbulence model on grids of increasing density. By doing this, changes to fluid properties due to the effect of grid sizes may be ascertained.

One such turbulence model involves the application of renormalized group analysis to the $k-\epsilon$ equation. This model was first presented by Yakhot and Orszag (1986) for application in turbulence modelling. It was further perfected by Choudhury (1993) with specific implementation in FLUENT. Application of the modified two-equation $k-\epsilon$ turbulence model for re-normalized groups with an enhanced wall function requires a minimum of ten cells in the region near the wall or shear layer where the turbulent Reynolds number is less than 200 and $y^{+}$is leses than 5 at the wall. (Fluent, Inc., 2003).

\subsubsection{Summary}

CFD is a well-developed tool for calculating and analyzing the dynamic features of fluid flow. Its application in simulation of aerosol samplers has been established by a number of studies. Finally, the recent advances in turbulence models in commercial CFD codes make it a viable tool for accurately predicting the sampling efficiency of personal aerosol samplers. 


\section{Chapter 3}

\section{Research Methods}

\subsection{Computational Fluid Dynamics}

\subsubsection{Governing Equations}

The primary research tool in this work is the FLUENT computational fluid dynamics software package (Fluent, Inc., Lebanon, NH). FLUENT contains a CFD solver based on a finite-volume formulation of integrable expressions of the governing equations of fluid flow, which are the Navier-Stokes equations. These equations consist of terms for conservation of mass, conservation of momentum, conservation of energy, and viscous stresses. 


\section{Conservation of Mass}

All properties of a fluid unit, i.e. a control volume, are related to its position, often defined in terms of $x, y$, and $z$ directional components, and time, $t$. Starting from a first-principles approach, such that mass is always conserved, the flux of the primary fluid property in terms of mass, i.e. density, across (into and out of) a control volume, defined by time and positional coordinates, is

$$
\frac{\partial \rho}{\partial t}+\frac{\partial(\rho u)}{\partial x}+\frac{\partial(\rho v)}{\partial y}+\frac{\partial(\rho w)}{\partial z}=0
$$

Equation 3.1 is obtained from the sum of partial derivatives of the density with respect to time for each component direction multiplied by the area of the particular face of the control volume it encompasses. For steady-state incompressible fluids, such as airflow into aerosol samplers (time-averaged), the above equation becomes

$$
\frac{\partial u}{\partial x}+\frac{\partial v}{\partial y}+\frac{\partial w}{\partial z}=0
$$

\section{Conservation of Momentum}

The sum of the surface forces on the control volume, or fluid particle, is equal to the change in momentum of the fluid particle. That is, the forces acting on the fluid particle, namely pressure forces, $p$, and viscous forces, $\tau_{i, j}$, will necessarily be equal to the resulting change of momentum. If mass is constant, as it is in this work, only velocity will change. 
An equation describing conservation of momentum is derived in a manner similar to that for mass, where the partial derivatives of the pressure and viscous forces with respect to position are summed for each face of the control volume (particle element) and then normalized by the volume of the element. The directional components of the momentum equation for $x, y$, and $z$ are,

$$
\begin{aligned}
\rho \frac{D u}{D t} & =\frac{\partial\left(-p+\tau_{x x}\right)}{\partial x}+\frac{\partial \tau_{y x}}{\partial y}+\frac{\partial \tau_{z x}}{\partial z}+S_{M x} \\
\rho \frac{D v}{D t} & =\frac{\partial \tau_{x y}}{\partial x}+\frac{\partial\left(-p+\tau_{y y}\right)}{\partial y}+\frac{\partial \tau_{z y}}{\partial z}+S_{M y} \\
\rho \frac{D w}{D t} & =\frac{\partial \tau_{x z}}{\partial x}+\frac{\partial \tau_{y z}}{\partial y}+\frac{\partial\left(-p+\tau_{z z}\right)}{\partial z}+S_{M z}
\end{aligned}
$$

where $S_{M i}$ is a contribution of body forces, such as gravity.

\section{Conservation of Energy}

The energy equation is based on the first of law of thermodynamics, which states that the change in the internal energy of the control volume is equal to the heat added into it, minus work done on the control volume (fluid particle). Mathematically, it is derived in a manner similar to the conservation of mass and momentum. However, it may consist of contributions of work from surface or body forces, as well as contribution of energy flux by heat conduction. The energy equation for incompressible flow is 


$$
\begin{array}{r}
\rho c \frac{D T}{D t}=\operatorname{div}(k \operatorname{grad} T) \\
+\tau_{x x} \frac{\partial u}{\partial x}+\tau_{y x} \frac{\partial u}{\partial y}+\tau_{z x} \frac{\partial u}{\partial z} \\
+\tau_{x y} \frac{\partial v}{\partial x}+\tau_{y y} \frac{\partial v}{\partial y}+\tau_{z y} \frac{\partial v}{\partial z} \\
+\tau_{x z} \frac{\partial w}{\partial x}+\tau_{y z} \frac{\partial w}{\partial y}+\tau_{z z} \frac{\partial w}{\partial z} \\
+S_{x}+S_{y}+S_{z}
\end{array}
$$

where $c$ is the specific heat and $k$ is heat conduction.

\section{Viscous Stresses and Navier-Stokes Equations}

For Newtonian fluids, a viscous stress on the control volume will result in a proportional amount of deformation. The viscous stress components are

$$
\begin{array}{r}
\tau_{x x}=2 \mu \frac{\partial u}{\partial x}+\lambda \operatorname{div} \vec{u} \\
\tau_{y y}=2 \mu \frac{\partial v}{\partial y}+\lambda \operatorname{div} \vec{u} \\
\tau_{z z}=2 \mu \frac{\partial w}{\partial z}+\lambda \operatorname{div} \vec{u} \\
\tau_{x y}=\tau_{y x}=\mu\left(\frac{\partial u}{\partial y}+\frac{\partial v}{\partial x}\right) \\
\tau_{x z}=\tau_{z x}=\mu\left(\frac{\partial u}{\partial z}+\frac{\partial w}{\partial x}\right) \\
\tau_{y z}=\tau_{z y}=\mu\left(\frac{\partial v}{\partial z}+\frac{\partial w}{\partial y}\right) .
\end{array}
$$


The viscous stress components are substituted into the momentum equation to arrive at the Navier-Stokes equations which, in simplified form, are

$$
\begin{aligned}
\rho \frac{D u}{D t} & =-\frac{\partial P}{\partial x}+\operatorname{div}(\mu \operatorname{grad} u)+S_{M x} \\
\rho \frac{D v}{D t} & =-\frac{\partial P}{\partial y}+\operatorname{div}(\mu \operatorname{grad} v)+S_{M y} \\
\rho \frac{D w}{D t} & =-\frac{\partial P}{\partial z}+\operatorname{div}(\mu \operatorname{grad} w)+S_{M z} .
\end{aligned}
$$

\section{Discretization}

The discretization is based on integration of the conservation form across the numerical grid in order to arrive at numerical difference equations. If fluid properties in 3.13 through 3.15 are replaced with a generic variable, $\phi$, then a further simplified form is obtained

$$
\frac{\partial(\rho \phi)}{\partial t}+\operatorname{div}(\rho \phi \vec{u})=\operatorname{div}(\Gamma \operatorname{grad} \phi)+S_{\phi}
$$

where $\Gamma$ is diffusion coefficient. Equation 3.16 is integrated across the grid (control volume) and the following (after Versteeg and Malalasekera, 1995) results

$$
\int_{C V} \frac{\partial(\rho \phi)}{\partial t} d V+\int_{C V} \operatorname{div}(\rho \phi \vec{u}) d V=\int_{C V} \operatorname{div}(\Gamma \operatorname{grad} \phi) d V+\int_{C V} S_{\phi} d V
$$


Discretized equations resulting from application of 3.17 to a computational grid (such as Fig. 3.1 based on Versteeg and Malalasekera (1995) and Ferziger and Peric' (1999)) for one-dimensional steady state diffusion are presented as

$$
\left(\frac{\Gamma_{e}}{\delta x_{P E}} A_{e}+\frac{\Gamma_{w}}{\delta x_{W P}} A_{w}-S_{p}\right) \phi_{P}=\left(\frac{\Gamma_{w}}{\delta x_{W P}} A_{w}\right) \phi_{W}+\left(\frac{\Gamma_{e}}{\delta x_{P E}} A_{e}\right) \phi_{E}+S_{u}
$$

The above implementation in FLUENT is at most second-order accurate in time and space. For the steady-state application in this work, only space coordinates are considered.

\section{Turbulence}

Solution of the time-dependent form of the Navier-Stokes equations (3.13 to 3.15), using the above discretization method on an extremely small grid, will capture the turbulent fluctuations, recirculation, and energy of a flow. However, this is impossible with current (2004) computational capabilities. Such an approach is called the direct numerical simulation, and may be possible someday if Moore's Law -"exponential growth in the number of transistors per integrated circuit per time"- continues to hold (Moore, 1965). However, for the foreseeable future, models of turbulence will be necessary.

The most common turbulence model, and the one applied in this work, is the twoequation $k-\epsilon$ model that approximates the production $(K)$ and dissipation $(\epsilon)$ of turbulent 


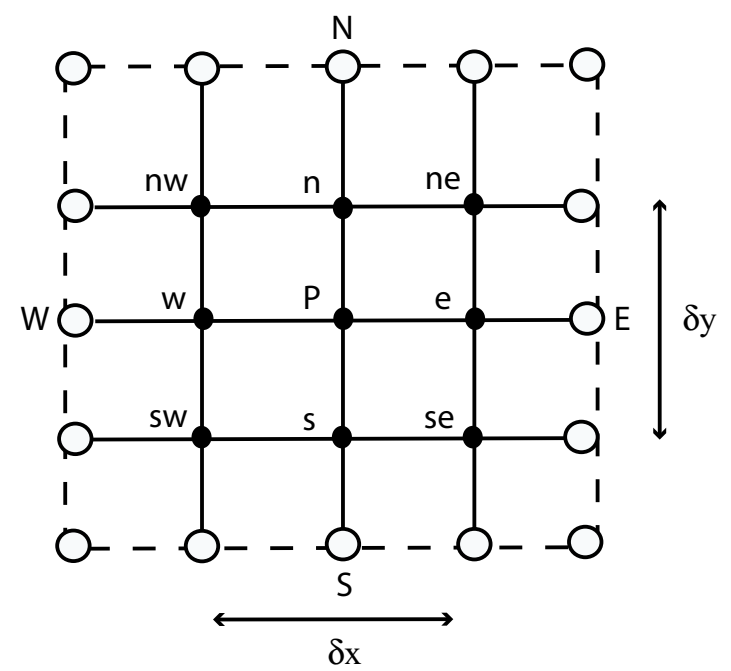

Figure 3.1: North-South-East-West grid used to demonstrate the discretizations used in CFD solvers.

energy from instantaneous Navier-Stokes equation (Reynolds-ensemble approach), which, presented in White (1991) for energy and dissipation, are

$$
\begin{array}{r}
\frac{D K}{D t} \approx \frac{\partial}{\partial x_{j}}\left(\frac{\nu_{t}}{\sigma_{k}} \frac{\partial K}{\partial x_{j}}\right)+\nu_{t} \frac{\partial \bar{u}_{i}}{\partial x_{j}}\left(\frac{\partial \bar{u}_{i}}{\partial x_{j}}+\frac{\partial \bar{u}_{j}}{\partial x_{i}}\right)-\epsilon \\
\frac{D \epsilon}{D t} \approx \frac{\partial}{\partial x_{j}}\left(\frac{\nu_{t}}{\sigma_{\epsilon}} \frac{\partial \epsilon}{\partial x_{j}}\right)+C_{1} \nu_{t} \frac{\epsilon}{K} \frac{\partial \bar{u}_{i}}{\partial x_{j}}\left(\frac{\partial \bar{u}_{i}}{\partial x_{j}}+\frac{\partial \bar{u}_{j}}{\partial x_{i}}\right)-C_{2} \frac{\epsilon^{2}}{K}
\end{array}
$$

where $\sigma_{K}=\nu_{t} / \nu_{k}, \sigma_{\epsilon}=\nu_{t} / \nu_{\epsilon}, n u_{t} \approx \frac{C_{\mu} K^{2}}{\epsilon}, C_{\mu}=0.09, C_{1}=1.44, C_{2}=1.92, \sigma_{k}=1.0$, and $\sigma_{\epsilon}=1.3$.

These relations and values are appropriate for many high-speed, high-Reynolds numbers flows away from walls. For the application in this work, i.e. low-speed, low-Reynolds number flows, and near walls, a more precise viscous treatment is needed. The method 
used in the FLUENT software is the renormalized group (RNG) analysis method, which is based on an analytically-derived differential formula (Choudhury, 1993; Fluent, Inc., 2003). The turbulent transport equations used in the RNG $k-\epsilon$ model in FLUENT, based on Yakhot and Orszag (1986) and Choudhury (1993), are

$$
\begin{aligned}
\frac{\partial}{\partial t}(\rho k)+\frac{\partial}{\partial x_{i}}\left(\rho k u_{i}\right) & =\frac{\partial}{\partial x_{j}}\left(\alpha_{k} \mu_{e f f} \frac{\partial k}{\partial x_{j}}\right) \\
& +G_{k}+G_{b}-\rho \epsilon+S_{k} \\
\frac{\partial}{\partial t}(\rho \epsilon)+\frac{\partial}{\partial x_{i}}\left(\rho \epsilon u_{i}\right)= & \frac{\partial}{\partial x_{j}}\left(\alpha_{\epsilon} \mu_{e f f} \frac{\partial \epsilon}{\partial x_{j}}\right) \\
& +C_{1 \epsilon} \frac{\epsilon}{k}\left(G_{k}+C_{3 \epsilon} G_{b}\right)-C_{2 \epsilon}^{*} \rho \frac{\epsilon^{2}}{k}-R_{\epsilon}+S_{\epsilon}
\end{aligned}
$$

where $G_{k}$ is generation of turbulence kinetic energy from mean velocity gradients, $G_{b}$ is generation of turbulence kinetic energy from buoyancy, $S$ represents source terms, $C_{1 \epsilon}=$ 1.42, $C_{2 \epsilon}=1.68, C_{3 \epsilon}$ is dependent on contribution of buoyancy, and $C_{2 \epsilon}^{*}=C_{2 \epsilon}+$ $\frac{C_{\mu} \rho \eta^{3}\left(1-\eta / \eta_{0}\right)}{1+\beta \eta^{3}}$. In order for this application of RNG theory to correctly model low-Reynoldsnumber flows, the following is used to calculate turbulent viscosity

$$
d\left(\frac{\rho^{2} k}{\sqrt{\epsilon \mu}}\right)=1.72 \frac{\hat{\nu}}{\sqrt{\hat{\nu}^{3}-1+C_{\nu}}} d \hat{\nu}
$$

where $\hat{\nu}=\mu_{e f f} / \mu$ and $C_{\nu} \approx 100$. Finally, there is an additional energy dissipation term in the RNG method not used in the standard $k-\epsilon$ equation, $R_{\epsilon}$, which is found from 


$$
R_{\epsilon}=\frac{C_{\mu} \rho \eta^{3}\left(1-\eta / \eta_{0}\right)}{1+\beta \eta^{3}} \frac{\epsilon^{2}}{k}
$$

with values $\eta=S k / \epsilon, \eta_{0}=4.38$, and $\beta=0.012$ (Fluent, Inc., 2003).

\section{Turbulence Intensity}

As previously mentioned, turbulence is calculated using the RNG $k-\epsilon$ model. Turbulence intensity at the inlets and within the sampler, provided it is less than about $10 \%$, should have little effect on the sampling efficiency for aerosol particles with Stokes numbers less than 1 (Vincent et al., 1985; Wiener et al., 1988), which is the case for these simulations. Turbulence intensity in the simulations is maintained at $5 \%$ at the inflow of the computational domain, and $3 \%$ inside the sampler based on experimental values of similar flows (Smith and Bird, 2002). The range of potential length scales were defined by estimates of the Kolmogorov microscales and experimental values (Vincent et al., 1985). They were defined to be 0.01 at the inflow to the domain (maximum), and equivalent to the minimum grid size at the respective sampler outlet, which was 0.0005 to $0.0010 \mathrm{~m}$. These lengths are expected to cover the range of possible scales that may evolve for these flow scenarios.

\section{Particle Trajectory Calculations}

To calculate particle trajectories, a Lagrangian reference frame is used where the forcebalance equation is integrated in order to find the direction and magnitude of the particle. 
The force balance equation is

$$
\frac{d u_{p}}{d t}=F_{D}\left(u-u_{p}\right)+\frac{g_{x}\left(\rho_{p}-\rho\right)}{\rho_{p}}+F_{x}
$$

where $F_{D}\left(u-u_{p}\right)$ is the drag per unit particle mass and

$$
F_{D}=\frac{18 \mu}{\rho_{p} d_{p}^{2}} \frac{C_{D} R e}{24}
$$

For turbulence treatments, the stochastic tracking (random walk) model is used to include the effect of instantaneous turbulent velocity fluctuations on the particle trajectories.

\subsubsection{Geometries for Simulations of Personal Aerosol Samplers}

Computational geometries of the samplers were built in the CAD software package, GAMBIT (Fluent, Inc., Lebanon, NH). Measurements from the actual samplers were made using calibrated digital calipers, and the resulting values from the measurements were used as the sizes for creating the computational geometries at a 1:1 scale.

\subsubsection{Boundary Conditions, Operating Conditions, and Computational Grids}

All calculations were conducted under environmental operating conditions that included the effect of gravity equal to $9.81 \mathrm{~m} / \mathrm{s}^{2}$ and the ambient pressure equal to 1 atmosphere 
(101, 325 Pascals). In addition, this work focused on steady-state solutions in order to simplify the simulations and approximate the time-averaging event of workplace and wind tunnel aerosol sampling.

Boundary conditions varied according to the model being solved and included the following:

(1) computational domain inflow velocity,

(2) grid density,

(3) order of solver,

(4) angle of orientation, and

(5) particle size.

For models involving the GSP sampler, a constant sampler exit velocity of $0.082525 \mathrm{~m} / \mathrm{s}$ was set for all of the models due to the manufacturer's recommendation that the sampler should be operated at $3.5 \mathrm{~L} / \mathrm{min}$. For all models involving the IOM sampler, a constant inlet velocity of $0.18 \mathrm{~m} / \mathrm{s}$ was set due to the manufacturer's recommendation that the sampler be operated at $2 \mathrm{~L} / \mathrm{min}$. For models involving thin-walled, sharp-edged samplers, inlet velocity was varied according to the freestream velocity, 0.5 or $1.0 \mathrm{~m} / \mathrm{s}$, so that isokinetic conditions were maintained.

Particle source faces were chosen based on their location in the geometry so that a desired spread of particles was achieved prior to a particular sample location. Typically ten particle injections were made for each particle size calculation. Particles were set to originate from faces or lines in amounts of 250 to 1000 per grouping. To account for turbulence effects, a 
stochastic treatment was applied to the particles where a minimum of three particles were released from the same location. The total number of particles released, depending on the simulation, ranged from several thousand to over 100,000.

Turbulence intensity was chosen based on values from laser-Doppler velocimetry data (Smith and Bird, 2002), which in freestream flow was typically around three to five\%. Turbulence length scale was chosen based on the local grid density and the requirement that the grid be no larger than the expected length scale.

\section{Solution Domain Grids}

Computational geometries of the solution domain were developed for samplers (dimensions shown in Figures 3.2 and 3.3) free in the air and mounted on a box-shaped bluff body at three orientations to the freestream flow, and with increasingly dense grids (see Figures 3.4 through 3.7). The wall function approach with enhanced wall treatment was applied to all simulations. For this approach the renormalized group (RNG) $k-\epsilon$ turbulence model was used with near-wall enhancement. This results in application of a standard or renormalized grouping $\kappa-\epsilon$ model for fully turbulent regions just outside the boundary layer transition zone, and then a change to application of the one-equation Wolfstein model, which is applied for the transition zone between the viscous sublayer and the fully turbulent region further away from the wall.

Boundary layers were applied to wall surfaces where appropriate such that the layers closest to the wall matched the finest mesh density within the respective grid. Boundary 
layers were typically 4 to 6 cells thick, although sometimes were greater than 10 , and grew at a rate of $105 \%$ to $115 \%$ until they reached the mesh external to the near-wall region. Boundary layer thickness was most influenced by determination of whether $y^{+}$was less than 5 at the surface, and whether there were at least 10 cells between where $R e_{T u r b}=200$ and the surface. Consideration was given for grid density in the near-wall region in order to nominally yield $y^{+}$values between 30 and 100 .

Evaluation of the computational domain at several grid densities was conducted in order to ensure that the size of grid cells did not have an effect on the solution. This was accomplished through qualitative plots, parameter space analysis, and application of the grid convergence index, which are discussed in detail later in this chapter.

Finally, the type of cell element used in the grid may have an influence on the results. Typically the hexahedrally shaped cell (ideally as a uniform cube) is used. However, for more rapid computational domain creation, tetrahedrally shaped cells have been used. For the class of flows in this work, preliminary examinations showed there is little difference $(<0.5 \%)$ between results obtained from hexahedral cells versus those obtained from tetrahedral cells. 


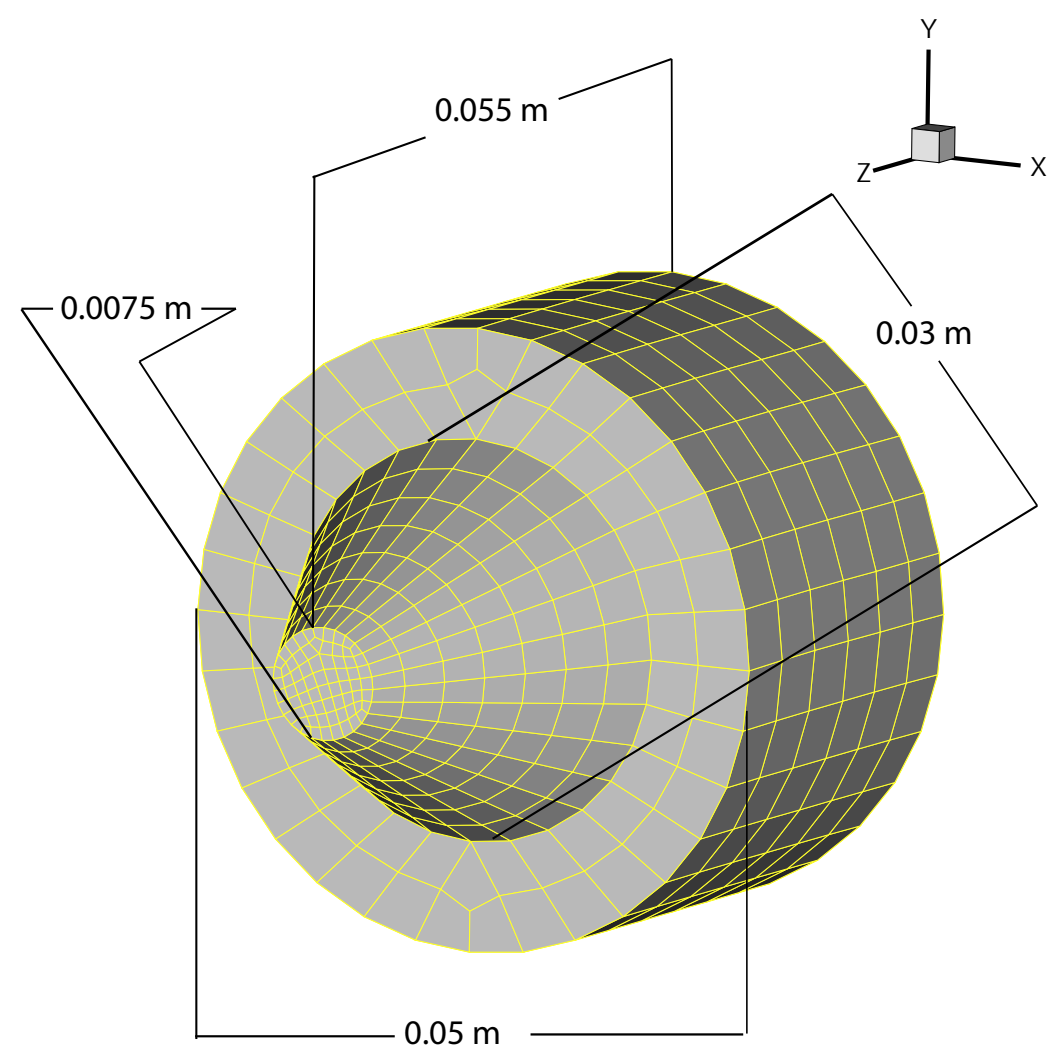

Figure 3.2: Dimensions of GSP sampler. 


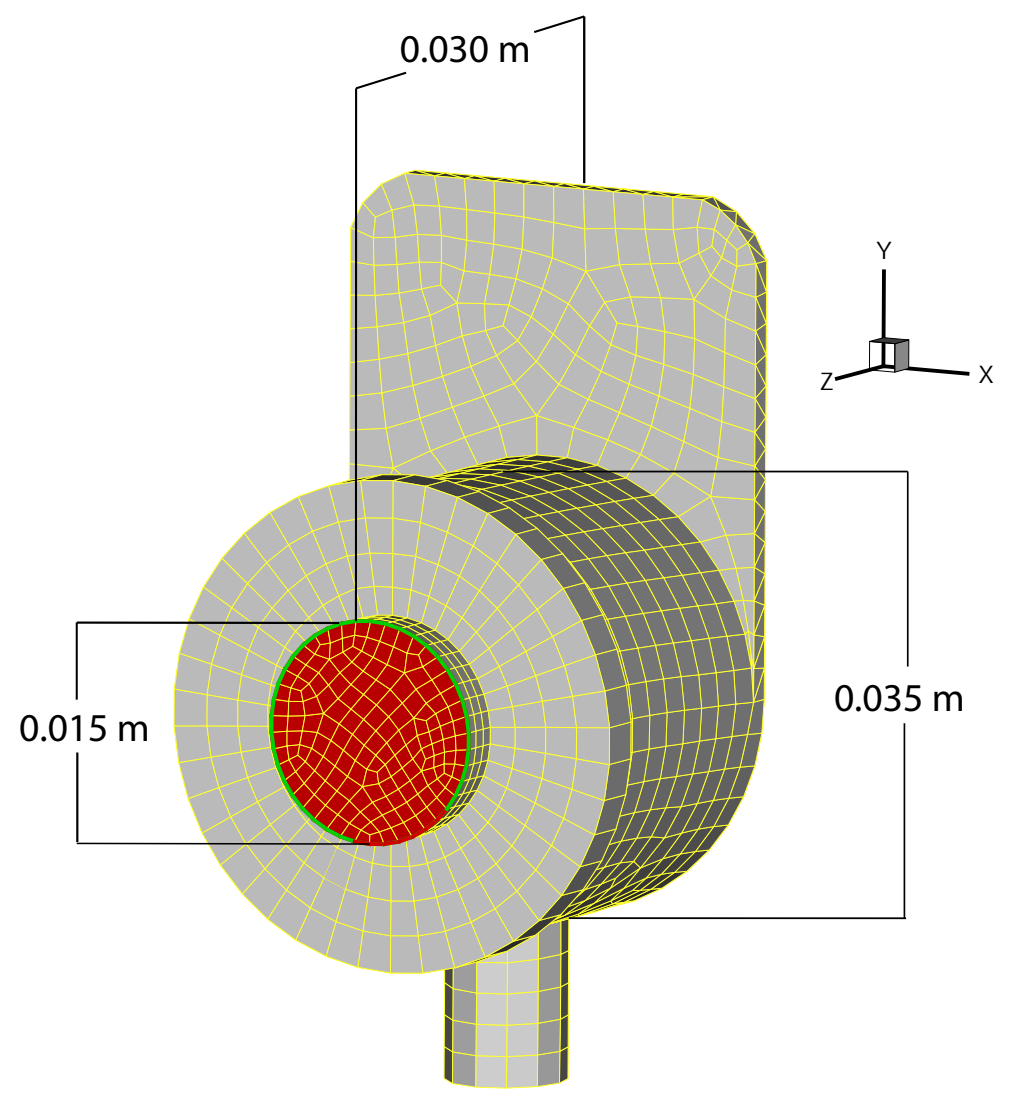

Figure 3.3: Dimensions of IOM sampler. 

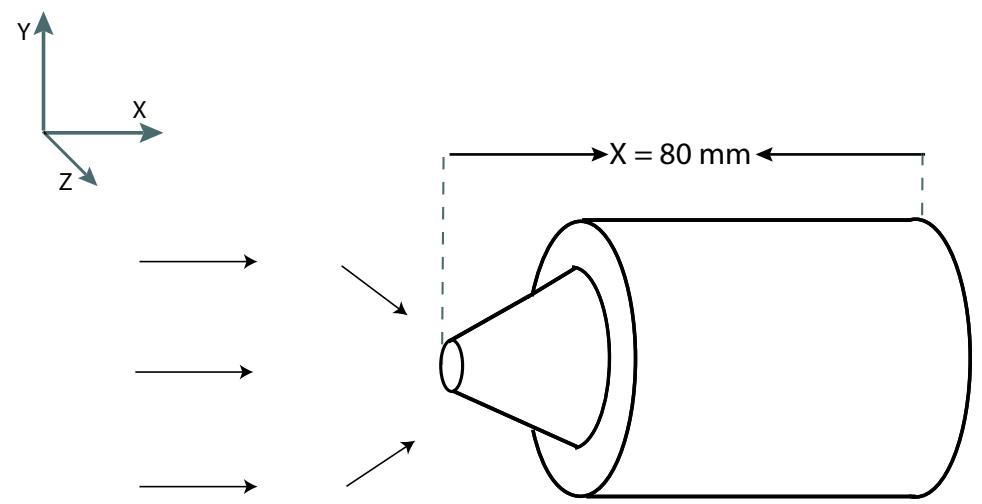

airflow direction

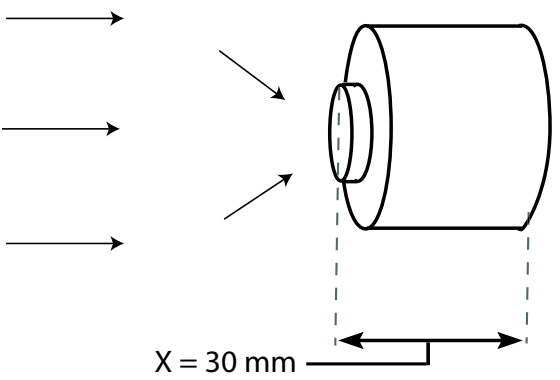

Figure 3.4: GSP and IOM samplers free in the air at 0-degree orientations with respect to freestream airflow direction. 

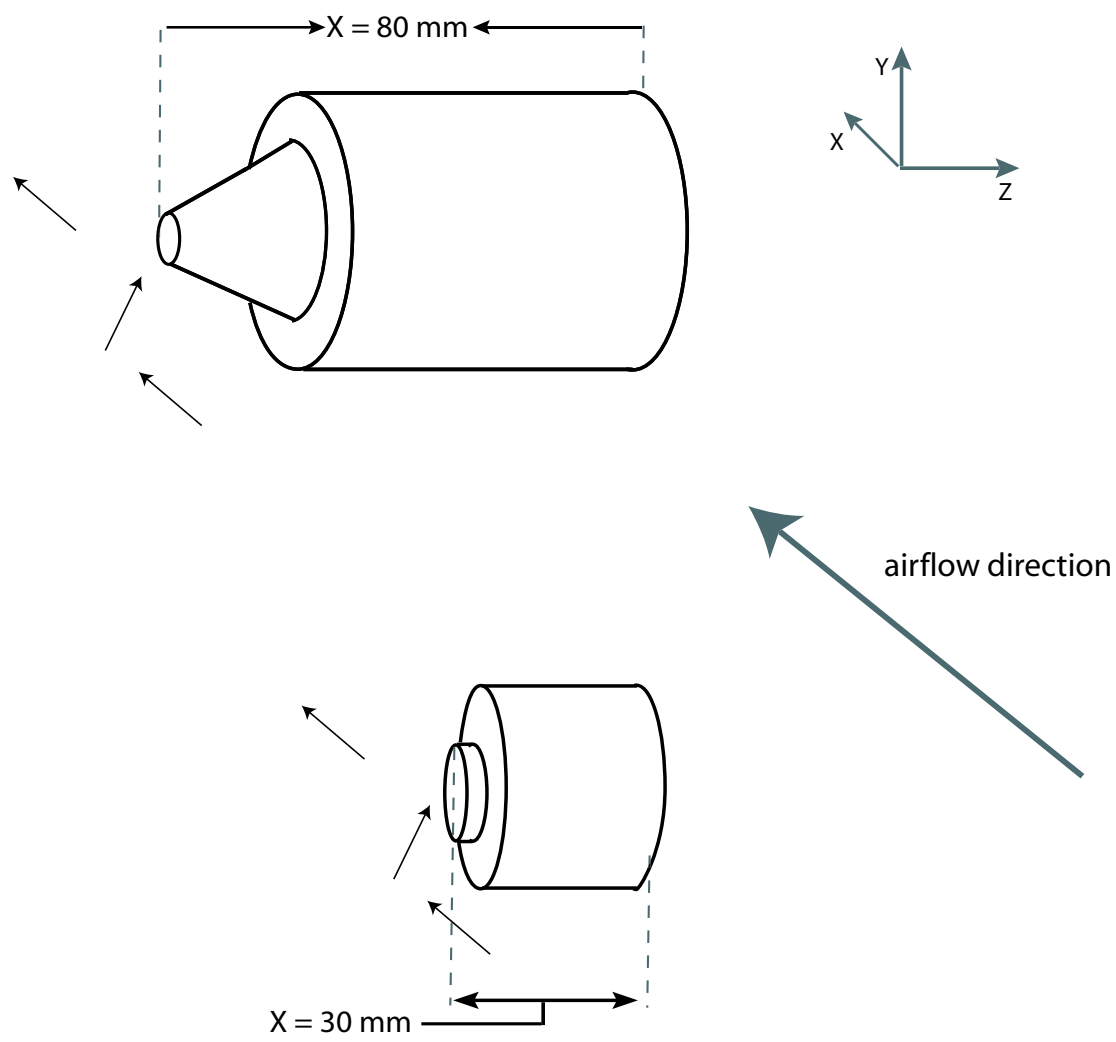

airflow direction
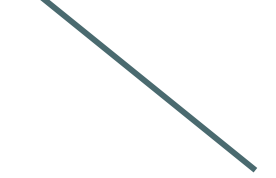

Figure 3.5: GSP and IOM samplers free in the air at 90-degree orientations with respect to freestream airflow direction. 

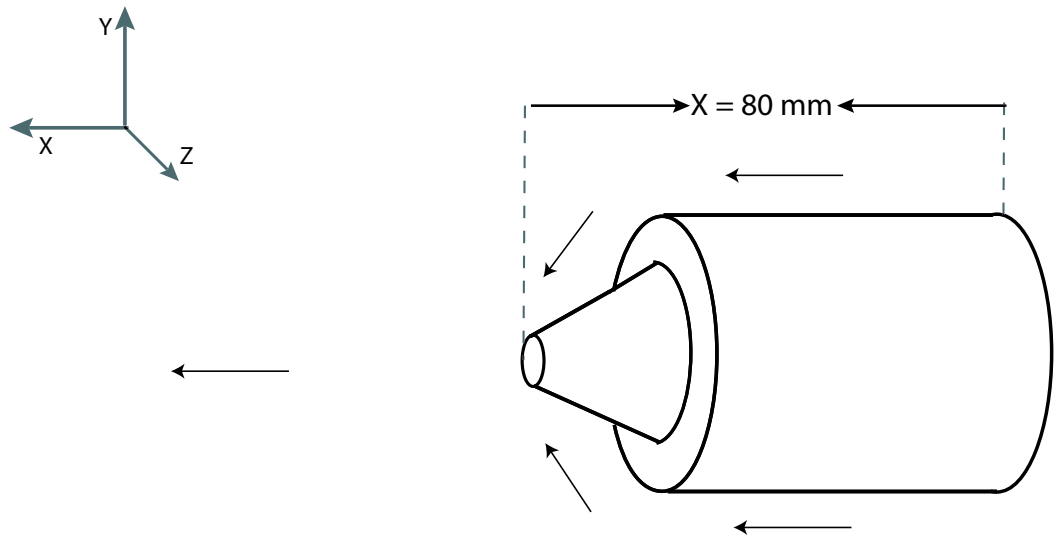

airflow direction
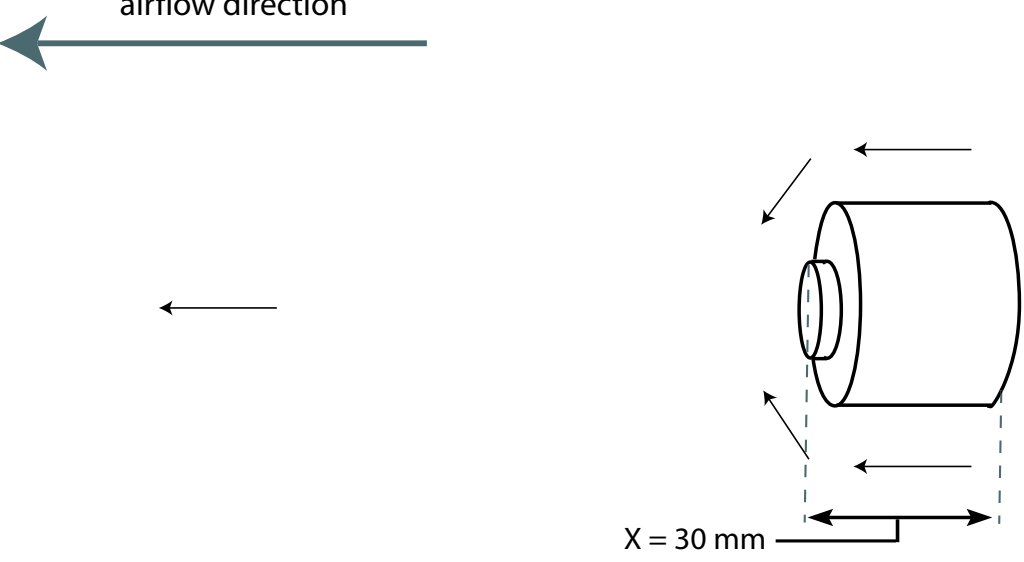

Figure 3.6: GSP and IOM samplers free in the air at 180-degree orientations with respect to freestream airflow direction. 


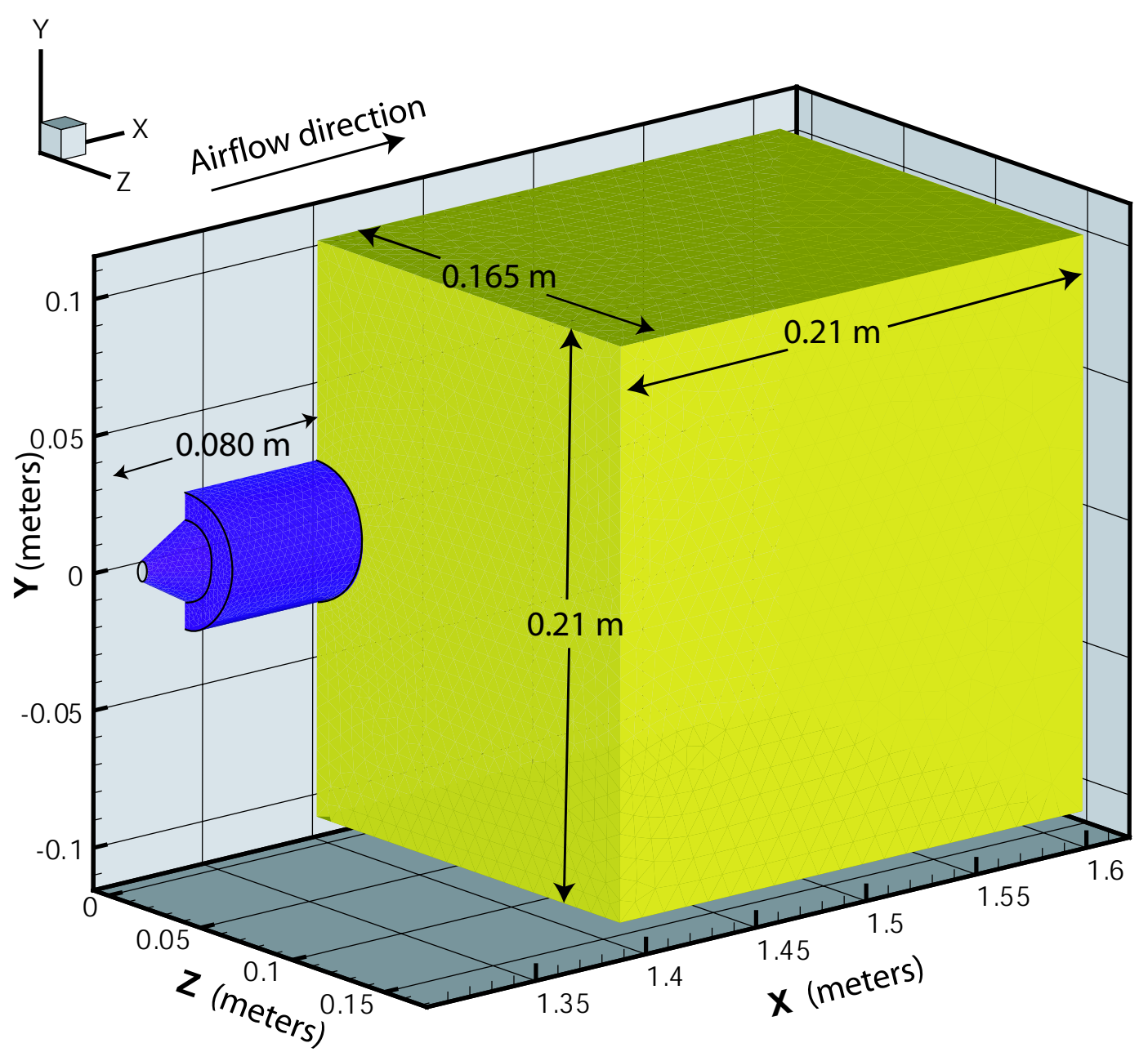

Figure 3.7: One-half symmetry diagram of GSP sampler mounted on the box-shaped bluff body at a 0-degree orientation. For 90-degree orientations, the sampler is moved to the side, and for 180-degree orientations it is moved to the back. The IOM sampler is situated in the same manner as the GSP. 


\section{Convergence of Simulations}

Models were given boundary conditions as described in the section above and iterated to convergence using the segregated FLUENT double-precision solver with the SIMPLE pressure correction method. Convergence was defined as "scaled-residual value $<10^{-3}$ " (Fluent, Inc., 2003). However, ideal convergence occurred when the residual values reach levels where they no longer decreased, i.e. where the residual plots reached a "flat-line stage."

Except for extremely dense grids, second-order upwinding was applied to the CFD models in order to allow an accurate and consistent calculation of the velocity field. In secondorder upwinding, the average is taken of two side-by-side cell center values and applied to the calculation of the cell face immediately downstream in order to yield accurate convection.

When appropriate, under-relaxation values were modified in order to achieve solution convergence. Under-relaxation values were typically kept low in order to allow the CFD solver to achieve accurate convergence at a reasonable rate. In equation $3.27, \phi$ is the value being calculated, pressure for example, and $\alpha$ is the under-relaxation factor. By keeping $\alpha$ relatively low, the impact of the change in the $\phi$ value was reduced, and thus the difference, $\delta \phi$, between the new value and the old value is small, which essentially means that the overall residual is lessened and convergence can be attained more quickly. 


$$
\phi=\phi_{\text {old }}+\alpha \delta \phi
$$

After each model was converged, grid independence was determined and the best model was selected. Particles were introduced into the flow fields of the best performing models via a random walk discrete phase method, sampling efficiency was calculated, and agreement with experiment was assessed.

The discrete random walk of the particle is used because the particle is "encouraged" to try many locations within the calculated flow domain. As particles are released, they are not allowed to enter the microregions that other particles have already populated. In this way, the particles are distributed over a greater area. For inhomogeneous flows that are diffusion dominated, small particles will begin to accumulate in low-turbulence regions. This situation, however, is not applicable to the current calculations, which are strongly advection dominated.

\section{Convergence Criteria}

Simulations were iterated to convergence for freestream and inlet velocities of 0.5 and $1.0 \mathrm{~m} / \mathrm{s}$, respectively. There has been discussion concerning the degree of convergence a simulation should achieve. However, a general rule of thumb in CFD is that a minimal convergence has been achieved when residuals have decreased by at least two orders of magnitude below initial conditions. In the simulations conducted in this work, convergence 
was defined as the residual (difference between previous and current value) "flat-lining," which typically occurred at better than 10 orders of magnitude below the initial conditions for most simulations (Figure 3.8).

Correct solutions were desired, but not to the degree that simulations required excessive computational time. To evaluate the impact of increasing the number of iterations, simulations were conducted for differing numbers of iterations to determine the degree of improvement. Preliminary research indicated that increasing the number of iterations from 2000 to 3800 (three days of additional computational time, yielding a 3 orders-ofmagnitude decrease in residuals) resulted in changes in velocity values of much less than $1 \%$, which suggests it is not useful to "overcalculate." Simulations that were not flat-lined converged after reaching at least 10 orders of magnitude below the initial condition were stopped and the results used as if the simulation had flat-line converged. 


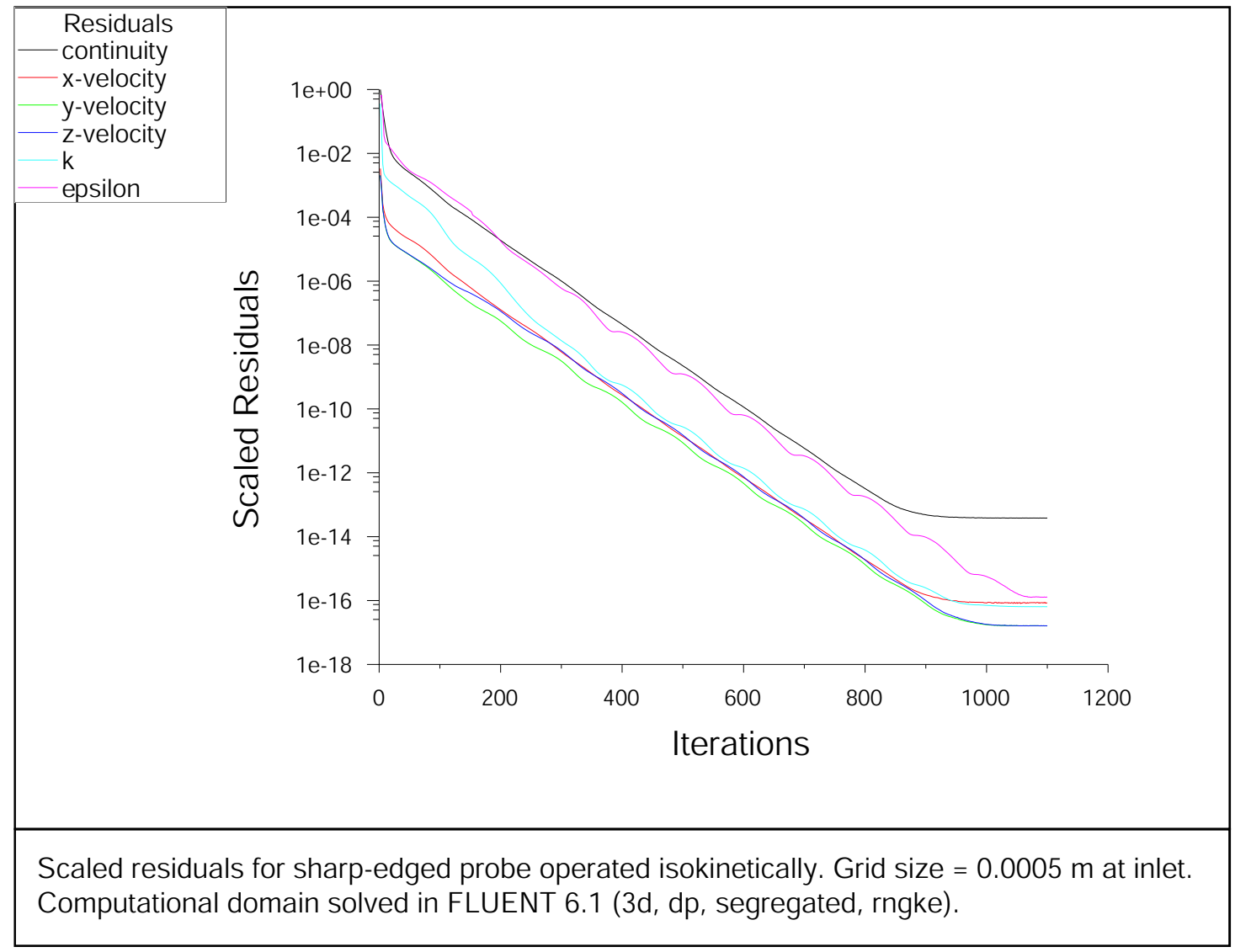

Figure 3.8: Expected convergence behavior of scaled residuals for simulations of interest. 


\subsubsection{Use of Reference Probe in Computational Domain}

Evaluation of the performance of personal samplers requires a testing environment where velocity, aerosol concentration, and turbulence are known and constant. In wind tunnel experiments, reference probes are used to sample the aerosol particles from the testing environment. The concentration determined by the sharp-edged sampling probe is considered to be the reference aerosol concentration and is used as a point of comparison for determining the sampling efficiency of a sampler being evaluated. Thus, in order to maintain consistency with experiments, the reference probe is also used in CFD simulation as a point of comparison for the GSP and IOM samplers.

It has been well established by experiment that the sampling efficiency for a thin-walled, sharp-edged sampler that is operated isokinetically in line with the freestream flow will collect aerosol particles up to at least $100 \mu \mathrm{m}$ with an efficiency of unity (Belyaev and Levin, 1974). 3-D meshes of probe samplers following the Belyaev and Levin criteria were created in GAMBIT (see Figure 3.9).

Reference probes created in GAMBIT were made to have a 5-degree angle of "sharpness," or less, which according to Belyaev and Levin (1974), together with a ratio of wall thickness to probe diameter less than 0.05 , will result in thin-walled sampling conditions. A probe having these dimensions and aspirating parallel to the oncoming flow (0-degree orientation), should give an aspiration efficiency of unity, thereby establishing a direct linkage from the true tunnel concentration to the concentration seen by the test samplers, which in this case are GSP and IOM samplers (See Figure 3.10). 


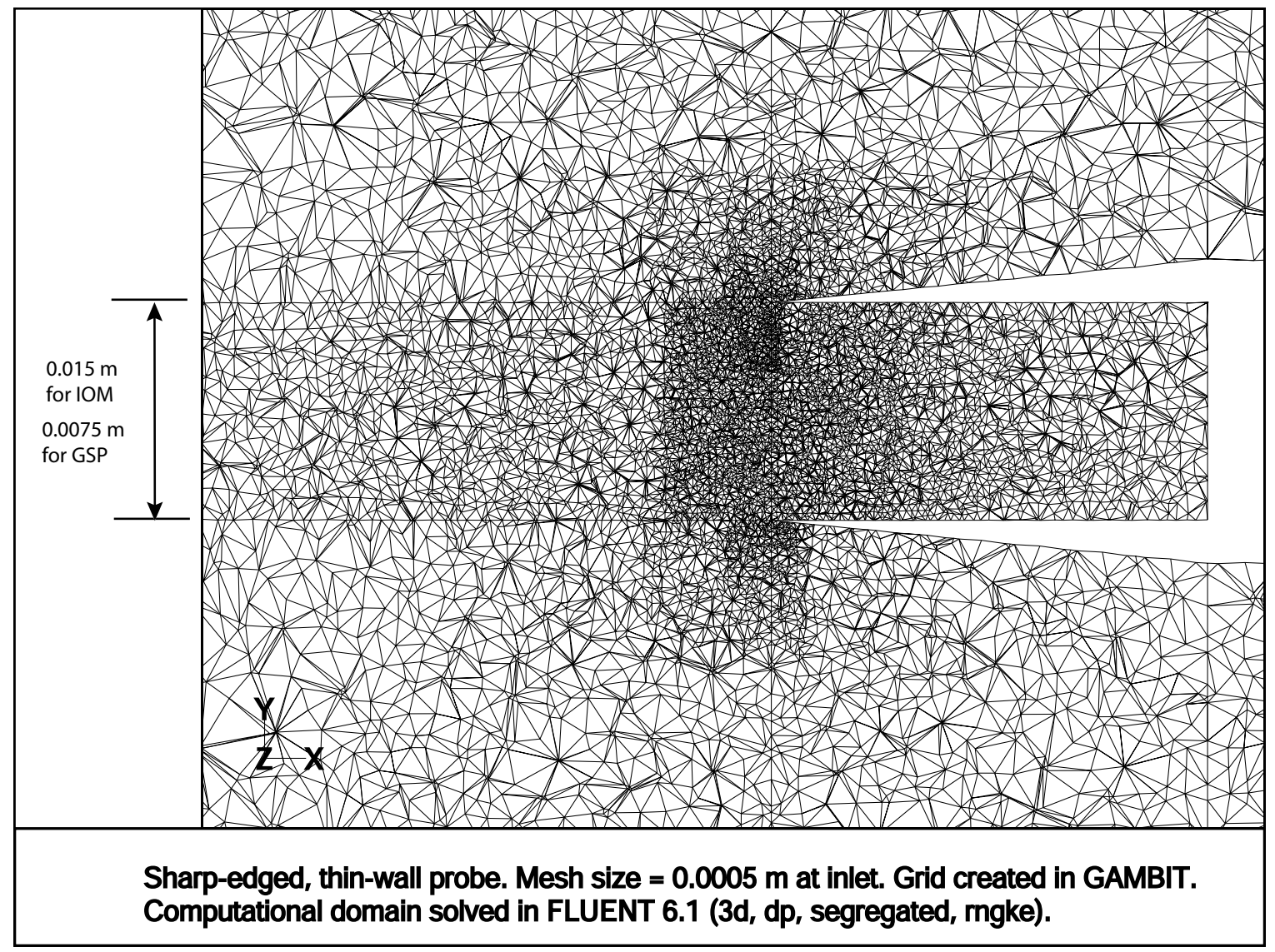

Figure 3.9: Centerline slice of 3D tetrahedral cell grid of sharp-edged probe at a 0-degree orientation to oncoming freestream flow. 


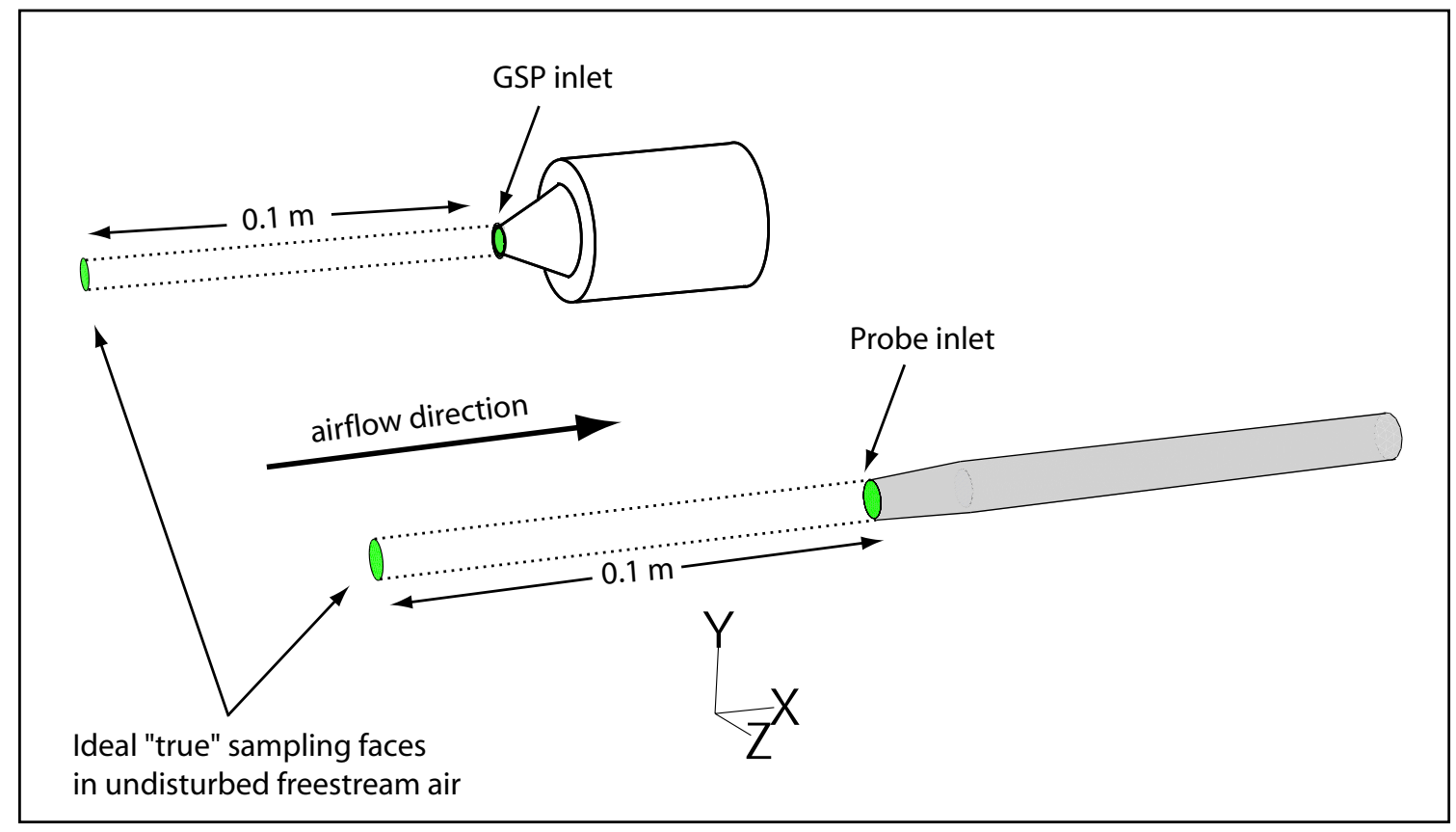

Figure 3.10: GSP personal sampler, reference probe sampler, and ideal sampling faces. 


\subsection{Resources}

\subsubsection{Commercial CFD Software Package}

CFD simulations were conducted using the FLUENT CFD software package together with the GAMBIT grid generator (Fluent, Inc., Lebanon, N.H.). The FLUENT/GAMBIT software was licensed on a year-to-year basis. Licenses were typically purchased so that up to two users could access the software simultaneously and then run simulations on up to 8 processors in a parallel configuration. Year-to-year licensing included technical and administrative support for the software, as well as access to upgrades.

\subsubsection{Graphics and Plotting Software}

Additional graphics and plotting software was necessary in order to analyze and view results of laser-Doppler velocimetry measurements and CFD simulations. Two packages that were commonly used included Tecplot (Amtec, Inc., Bellevue, WA) and Ensight (Computational Engineering International Apex, North Carolina). Tecplot was used for plotting data from experiments as well as the results from simulation. Ensight was used for visualizing the results of CFD simulations conducted in parallel on many processors. 


\subsubsection{Computer Hardware}

Simulations were conducted on two computers over the course of the research period. The first was a SUN-6000 Enterprise Server (SUN) with eighteen 333 megahertz SPARC processors and 14 gigabytes of memory in a shared-memory, 64-bit architecture. The operating system (O.S.) was SUN Solaris version 2.6, which is 32-bit. Even though the architecture on the SUN is 64 bit, the O.S. limited it to 32 bits and a maximum of 2 gigabytes of memory per processor. The second computer was a Linux Networx Cluster with eight 3.06 gigahertz XEON processors and 16 gigabytes of memory. This architecture is PC-based, therefore it is also limited to 32 bits and 2 gigabytes of memory per processor. Please see Appendix B for further details of computer hardware.

\subsection{Data Analysis}

Results of CFD simulations are viewed in "post-processors." These are software packages that allow viewing of the pressure, velocity, and particle trajectory calculations in the appropriate grid, whether it be 2D, 3D, and/or time-dependent. FLUENT has a built-in post-processor that allows viewing of results. In addition, the ENSIGHT (Computational Engineering International, Inc., Apex, NC) and TECPLOT (Tecplot, Inc., Bellevue, WA) post-processors may also be used, depending on the data type and the number of processors used to solve the problems. 


\subsubsection{Qualitative Plots}

The numerical data generated from this research are deterministic. This means no statistical analyses may be conducted on them, and no statistical comparisons may be made between the numerical results and existing experimental data. However, there are a number of useful comparative analyses possible, and often the most revealing is simply to plot the simulation and experimental results together on a graph in order to make qualitative conclusions.

\subsubsection{Percent Relative Difference}

A rather helpful comparison is the percent relative difference, or disagreement, calculation, which is

$$
A \%=\frac{(\mathrm{SIM}-\mathrm{EXP})}{\mathrm{EXP}} X 100
$$

where $A \%$ is the percent level of agreement, SIM is the value from simulation, and EXP is the corresponding value from experiment. Using the above equation for a large amount of data, agreement maps in time and space can be made, which make it possible to rapidly locate high disagreements, relative to user-defined metrics. 


\subsubsection{Parameter Space Analysis}

Parameter space analysis is based on a non-statistical application of factorial analysis. Parameters are broken into two levels, high and low. The simulations are conducted for each of the parameters at their specific parameter levels. For illustrative purposes, results from the simulations can be plotted in a multi-dimensional space in order to see where they are with respect to one another (See Figure 3.11).

For example, if the simulation comparison is of parameters including grid density, order of numerical method, and analysis location, there are three parameters, each at two levels. They include two grids (medium and fine), two solvers (1st- and 2nd-order), and two comparison locations (center-point and center-band) yielding a total of eight full combinations of parameters and parameter levels. Assigning half the parameter levels as a "low level" and giving them a value of -1 (see '-' in Table 3.1) and assigning the other half as "high level" and giving them a value of +1 (see ' + ' in Table 3.1) allows us to make a factorial-like comparison between simulation results.

In Table 3.1, the parameters are listed with their levels below them. In the "Result" column is the combination of the three primary levels shown as "lll" for "low, low, low," "lhl" for "low, high, low," and "hhh" for "high, high, high." Figure 3.9 demonstrates this concept three dimensionally. To determine the average delta due to the particular factor, the difference of values in vertical alignment are found and then the average is taken. Thus for the case of the grid parameter, 
Table 3.1: Low- and high-level coding map for parameter space analysis.

\begin{tabular}{|c|c|c|c|c|}
\hline Parameter & Grid & Order & Spread & Result \\
\hline & & & & \\
& - & - & - & $1 l l$ \\
& - & - & + & llh \\
& - & + & - & lhl \\
& - & + & + & lhh \\
& + & - & - & hll \\
& + & - & + & hlh \\
& + & + & - & hhl \\
& + & + & + & hhh \\
Avg. Delta & D1 & D2 & D3 & \\
& & & & \\
\hline
\end{tabular}

$$
\begin{aligned}
D 1= & {[(-1 * l l l)+(-1 * l l h)+(-1 * l h h)+(-1 * l h l)} \\
& +(1 * h l l)+(1 * h l h)+(1 * h h h)+(1 * h h l)] / 4
\end{aligned}
$$

where D1 represents average delta. The same form is used for each parameter and parameter combination. It must be reiterated that this procedure is not a statistical method. It is best used with qualitative plotting and other quantitative analysis, such as the Grid Convergence Index method, in order to reinforce observations about trends in the results. 


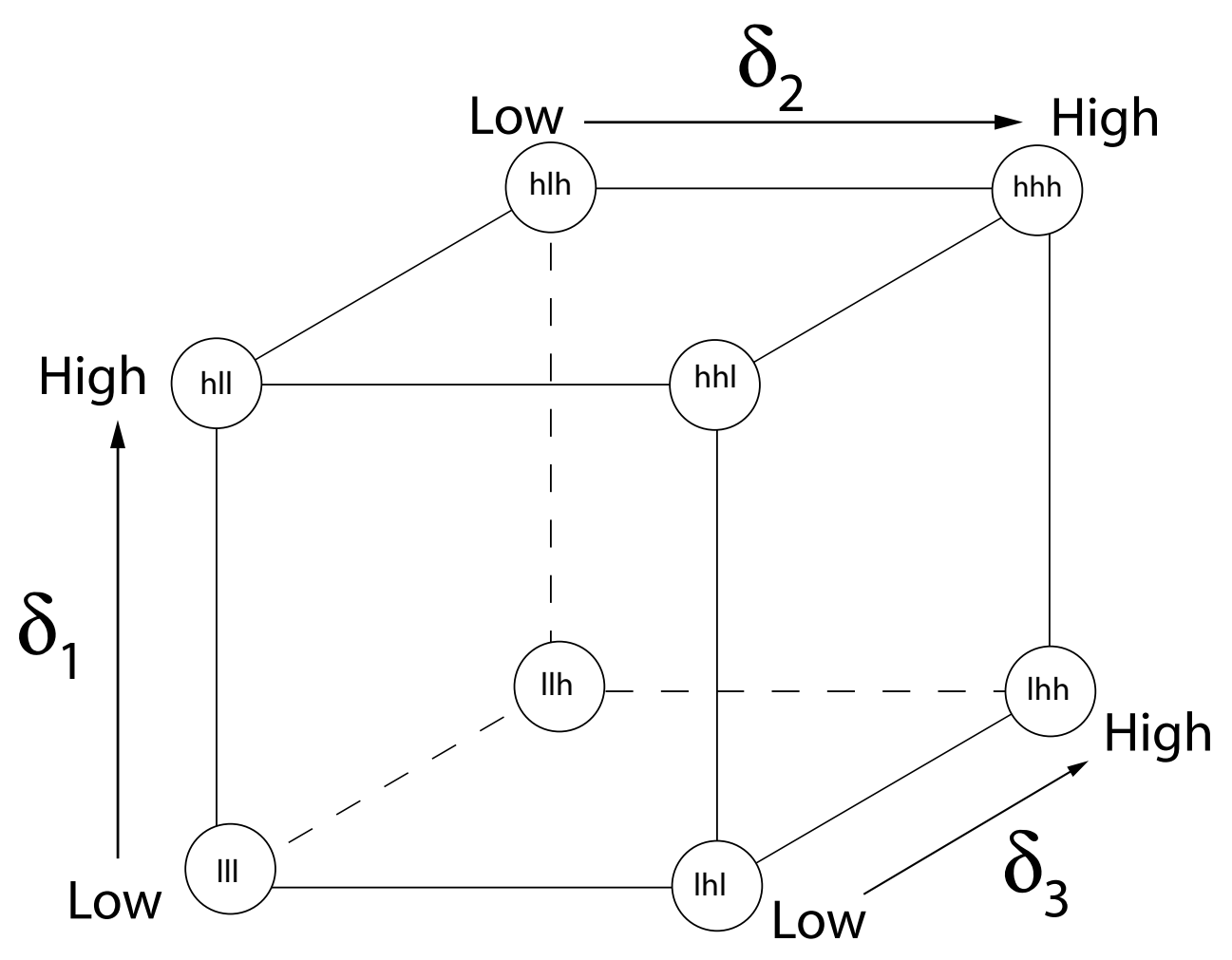

Figure 3.11: 3D representation of three calculation parameters each at two levels. 


\subsubsection{Grid Convergence Studies}

A more quantitative evaluation of the influence of the grid densities can be found using the Grid Convergence Index (GCI) method (after Roache, 1998). This method is based on Richardson Extrapolation and allows an estimate of the uncertainty in the calculation results due to the grid, provided there are measurable differences between the grids. This application of grid convergence study incorporates the percent relative difference between results from different grids and normalizes them by the factor of scaling from one grid to the next. This method of grid convergence study is best applied when solutions exist for differing orders of the numerical methods and for several grid densities. The GCI method, applied for an identical computational grid domain of two different densities is

$$
G C I[\text { dense } 2 / \text { dense } 1]=\mathrm{F}_{\mathrm{s}} \frac{|\epsilon|}{\mathrm{r}^{\mathrm{p}}-1}
$$

where "dense2" is the more dense grid; "dense1" is the less dense grid; $F_{s}$ is a "factor of safety," according to Roache (1998), which is usually 3, but sometimes smaller; $\epsilon$ is the relative difference between scalar values from the different grids; $r$ is the ratio of differing grid densities, i.e. $r=$ (more dense grid)/(less dense grid); and $p$ is the apparent order of the numerical method from which the solution was obtained. The characteristic density, $h$, of the grid ("dense1" and "dense2" from above) can be obtained according to the Journal of Fluids Engineering editorial policy (JFE, 2004) which is

$$
h=\left[\frac{1}{N} \sum_{i=1}^{N}\left(\Delta V_{i}\right)\right]^{1 / 3}
$$


where $\Delta V_{i}$ is the volume of the $i^{t h}$ cell and $N$ is the total number of cells in the domain used for the simulation.

\subsection{Summary of Research Methods}

1. Geometries of GSP and IOM Inhalable samplers are measured with digital calipers to accuracy of $10^{-4} \mathrm{~m}$.

2. Measurements are used to create computational grids of samplers in GAMBIT for 0-, 90-, and 180-degree orientations to freestream flow.

3. Meshes of varying density (from the same domain) are exported to FLUENT with smallest cell sizes of $0.0005,0.0010,0.0015$, and $0.0020 \mathrm{~m}$.

4. Boundary conditions are assigned to the meshes: inflow velocities of 0.5 and $1.0 \mathrm{~m} / \mathrm{s}$, GSP outflow of $0.08252 \mathrm{~m} / \mathrm{s}$, IOM outflow of $0.18 \mathrm{~m} / \mathrm{s}$, inflow turbulence intensity of $5 \%$ and length scale of $0.01 \mathrm{~m}$, GSP and IOM turbulence intensities of 3\% and length scales equal to smallest cell sizes (see 3.), external boundary of 0 shear, gravity equal to $9.81 \mathrm{~m} / \mathrm{s}$, and pressure of 101,325 $\mathrm{Pa}$.

5. Solutions are calculated using the 1st- and 2nd-order numerical discretizations available in FLUENT.

6. Simulations are iterated to convergence such that there is a 10 -order of magnitude decrease in residuals from initial conditions. 
7. After convergence, analysis of the computational domain is conducted to determine independence of calculation parameters using qualitative plots, parameter space analysis, and grid convergence index method.

8. Particle trajectories are calculated from velocity fields solved on independent grids.

9. Validation and calibration is conducted. 


\section{Chapter 4}

\section{Results}

\subsection{CFD Simulations}

CFD simulations were made of airflow behavior around and into the computational geometries of three samplers: GSP, IOM, and the sharp-edged reference probe. Results from simulations of the GSP and probe samplers were used to identify optimized calculation and analysis parameters for the class of flows most commonly seen by aerosol sampling devices.

Simulations were conducted with the boundary conditions described in the research methods chapter using 1st- and 2nd-order CFD solvers in the FLUENT software (see Tables 4.1 and 4.2). The CFD results were evaluated by examining calculated cell values from a number of different simulations in comparison to each other. This was done for the 
sharp-edged reference probe and the GSP sampler since they are representative of the class of flows expected for a wide variety of air sampling devices. In this work, the sharp-edged reference probe has inlet velocities equal to freestream, which were 0.5 and $1.0 \mathrm{~m} / \mathrm{s}$. The GSP has an inlet velocity of $1.5 \mathrm{~m} / \mathrm{s}$. Most air samplers have inlet velocities in the range between about 0.2 and $2 \mathrm{~m} / \mathrm{s}$. 
Table 4.1: Calculation parameters and levels used for CFD simulations in this work.

\begin{tabular}{|c|c|c|c|c|c|}
\hline Sampler & $\begin{array}{c}\text { Orientation } \\
\text { (degrees) }\end{array}$ & $\begin{array}{c}\text { Freestream } \\
\text { Windspeed } \\
(\mathrm{m} / \mathrm{s})\end{array}$ & $\begin{array}{l}\text { Order of } \\
\text { Method }\end{array}$ & $\begin{array}{c}\text { Grid } \\
\text { Density }\end{array}$ & $\begin{array}{l}\text { Analysis } \\
\text { Locations }\end{array}$ \\
\hline Probe & 0 & 0.5 & $1 \mathrm{st}$ & Coarse & Center, Wall \\
\hline Probe & 0 & 0.5 & $1 \mathrm{st}$ & Medium & Center, Wall \\
\hline Probe & 0 & 0.5 & $1 \mathrm{st}$ & Fine & Center, Wall \\
\hline Probe & 0 & 0.5 & $1 \mathrm{st}$ & Superfine & Center, Wall \\
\hline Probe & 0 & 0.5 & 2nd & Coarse & Center, Wall \\
\hline Probe & 0 & 0.5 & 2 nd & Medium & Center, Wall \\
\hline Probe & 0 & 0.5 & 2nd & Fine & Center, Wall \\
\hline Probe & 0 & 0.5 & 2 nd & Superfine & Center, Wall \\
\hline Probe & 0 & 1.0 & $1 \mathrm{st}$ & Coarse & Center, Wall \\
\hline Probe & 0 & 1.0 & $1 \mathrm{st}$ & Medium & Center, Wall \\
\hline Probe & 0 & 1.0 & $1 \mathrm{st}$ & Fine & Center, Wall \\
\hline Probe & 0 & 1.0 & $1 \mathrm{st}$ & Superfine & Center, Wall \\
\hline Probe & 0 & 1.0 & 2 nd & Coarse & Center, Wall \\
\hline Probe & 0 & 1.0 & 2nd & Medium & Center, Wall \\
\hline Probe & 0 & 1.0 & 2 nd & Fine & Center, Wall \\
\hline Probe & 0 & 1.0 & 2nd & Superfine & Center, Wall \\
\hline GSP & 0 & 0.5 & $1 \mathrm{st}$ & Coarse & Center, Wall \\
\hline GSP & 0 & 0.5 & $1 \mathrm{st}$ & Medium & Center, Wall \\
\hline GSP & 0 & 0.5 & $1 \mathrm{st}$ & Fine & Center, Wall \\
\hline GSP & 0 & 0.5 & $1 \mathrm{st}$ & Superfine & Center, Wall \\
\hline GSP & 0 & 0.5 & 2nd & Coarse & Center, Wall \\
\hline GSP & 0 & 0.5 & 2 nd & Medium & Center, Wall \\
\hline GSP & 0 & 0.5 & 2 nd & Fine & Center, Wall \\
\hline GSP & 0 & 0.5 & 2 nd & Superfine & Center, Wall \\
\hline GSP & 0 & 1.0 & $1 \mathrm{st}$ & Coarse & Center, Wall \\
\hline GSP & 0 & 1.0 & 1 st & Medium & Center, Wall \\
\hline GSP & 0 & 1.0 & $1 \mathrm{st}$ & Fine & Center, Wall \\
\hline GSP & 0 & 1.0 & $1 \mathrm{st}$ & Superfine & Center, Wall \\
\hline GSP & 0 & 1.0 & 2nd & Coarse & Center, Wall \\
\hline GSP & 0 & 1.0 & 2nd & Medium & Center, Wall \\
\hline GSP & 0 & 1.0 & 2 nd & Fine & Center, Wall \\
\hline GSP & 0 & 1.0 & 2nd & Superfine & Center, Wall \\
\hline
\end{tabular}


Table 4.2: Additional calculation parameters and levels for CFD simulations.

\begin{tabular}{|c|c|c|c|c|c|}
\hline Sampler & $\begin{array}{c}\text { Orientation } \\
\text { (degrees) }\end{array}$ & $\begin{array}{c}\text { Freestream } \\
\text { Windspeed } \\
(\mathrm{m} / \mathrm{s})\end{array}$ & $\begin{array}{c}\text { Order of } \\
\text { Method }\end{array}$ & $\begin{array}{c}\text { Grid } \\
\text { Density }\end{array}$ & $\begin{array}{c}\text { Analysis } \\
\text { Locations }\end{array}$ \\
\hline GSP & 90 & 0.5 & 1st & Fine & Center, Wall \\
GSP & 90 & 1.0 & 1st & Fine & Center, Wall \\
GSP & 90 & 0.5 & 2nd & Fine & Center, Wall \\
GSP & 90 & 1.0 & 2nd & Fine & Center, Wall \\
GSP & 180 & 0.5 & 1st & Fine & Center, Wall \\
GSP & 180 & 1.0 & 1st & Fine & Center, Wall \\
GSP & 180 & 0.5 & 2nd & Fine & Center, Wall \\
GSP & 180 & 1.0 & 2nd & Fine & Center, Wall \\
IOM & 0 & 0.5 & 2nd & Fine & Center, Wall \\
IOM & 0 & 1.0 & 2nd & Fine & Center, Wall \\
IOM & 90 & 0.5 & 2nd & Fine & Center, Wall \\
IOM & 90 & 1.0 & 2nd & Fine & Center, Wall \\
IOM & 180 & 0.5 & 2nd & Fine & Center, Wall \\
IOM & 180 & 1.0 & 2nd & Fine & Center, Wall \\
GSP on Box & 0 & 0.5 & 2nd & Fine & Center, Wall \\
GSP on Box & 0 & 1.25 & 2nd & Fine & Center, Wall \\
GSP on Box & 0 & 2.0 & 2nd & Fine & Center, Wall \\
GSP on Box & 90 & 0.5 & 2nd & Fine & Center, Wall \\
GSP on Box & 90 & 1.25 & 2nd & Fine & Center, Wall \\
GSP on Box & 90 & 2.0 & 2nd & Fine & Center, Wall \\
GSP on Box & 180 & 0.5 & 2nd & Fine & Center, Wall \\
GSP on Box & 180 & 1.25 & 2nd & Fine & Center, Wall \\
GSP on Box & 180 & 2.0 & 2nd & Fine & Center, Wall \\
IOM on Box & 0 & 0.5 & 2nd & Fine & Center, Wall \\
IOM on Box & 0 & 1.25 & 2nd & Fine & Center, Wall \\
IOM on Box & 0 & 2.0 & 2nd & Fine & Center, Wall \\
IOM on Box & 90 & 0.5 & 2nd & Fine & Center, Wall \\
IOM on Box & 90 & 1.25 & 2nd & Fine & Center, Wall \\
IOM on Box & 90 & 2.0 & 2nd & Fine & Center, Wall \\
IOM on Box & 180 & 0.5 & 2nd & Fine & Center, Wall \\
IOM on Box & 18 & 1.25 & 2nd & Fine & Center, Wall \\
IOM on Box & 180 & 2.0 & 2nd & Fine & Center, Wall \\
& & & & & \\
\hline
\end{tabular}




\subsubsection{Analysis of Simulation Results for Probe and GSP Samplers}

As with all CFD simulations, it was very important to know if changes in the calculation parameters would lead to changes in results. In order to determine this, qualitative and quantitative analyses were conducted for grid density, order of numerical method, and location from where results were extracted. This was done in three ways: (1) by plotting the results in qualitative graphs; (2) through application of a parameter space analysis method; and (3) by application of a grid convergence study.

\section{Qualitative Plots}

The parameter independence evaluation was conducted for the sharp-edged reference probe such that the velocity magnitudes were compared in qualitative plots of velocity magnitude. Figures 4.1 and 4.2 show velocity magnitudes taken from identical $\mathrm{x}, \mathrm{y}$, and $\mathrm{z}$ locations from three different grid densities of computational grids of one half of the probe inlet. Grid densities were determined from equation 3.31. From the plots, it appears that there are small differences between the grid densities. This was confirmed through comparison of the maximum and minimum values from the different grid densities at the same coordinate location by calculating the percent relative error at each measurement point, which was found to be no larger than $2 \%$ in all cases. 


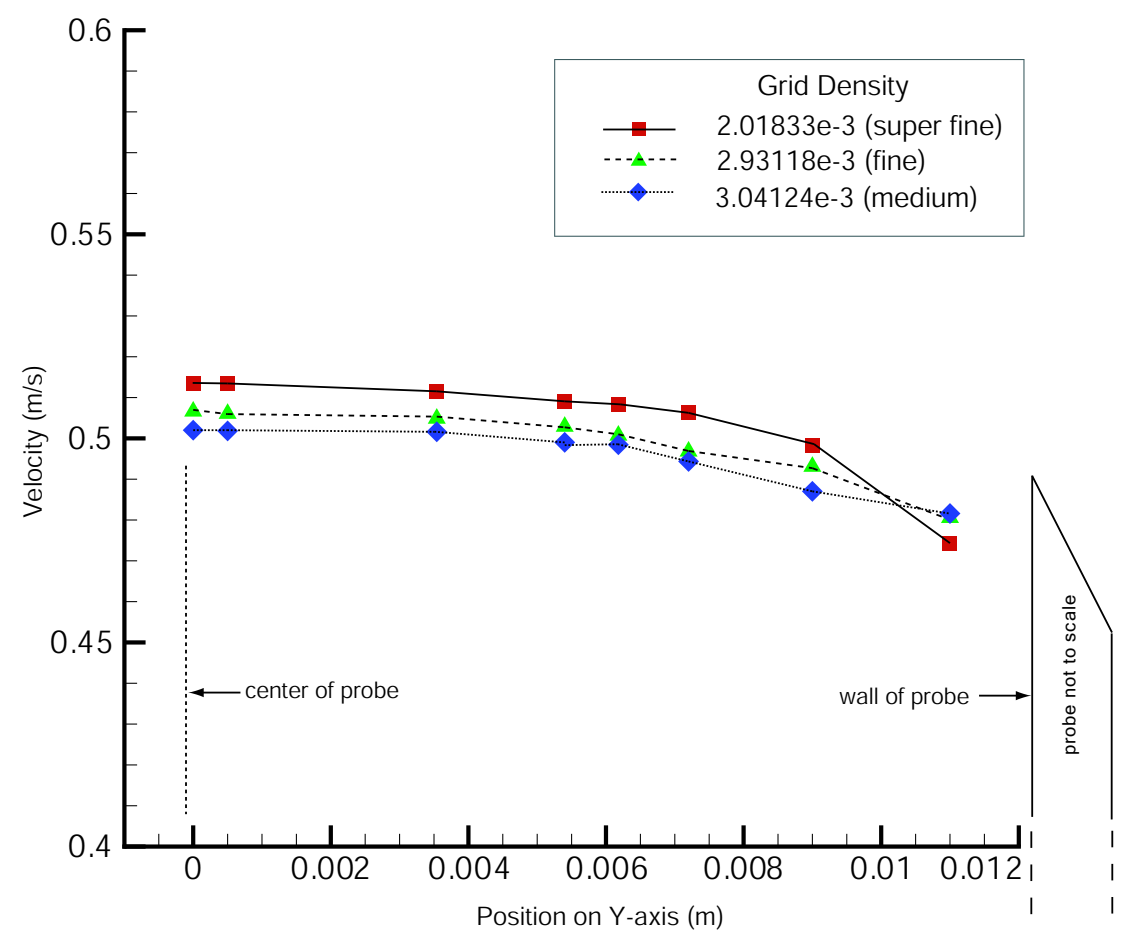

Figure 4.1: Velocity values of three grid densities across inlet of isokinetic sampler for 0.5 $\mathrm{m} / \mathrm{s}$ freestream and sampler inlet velocities. 


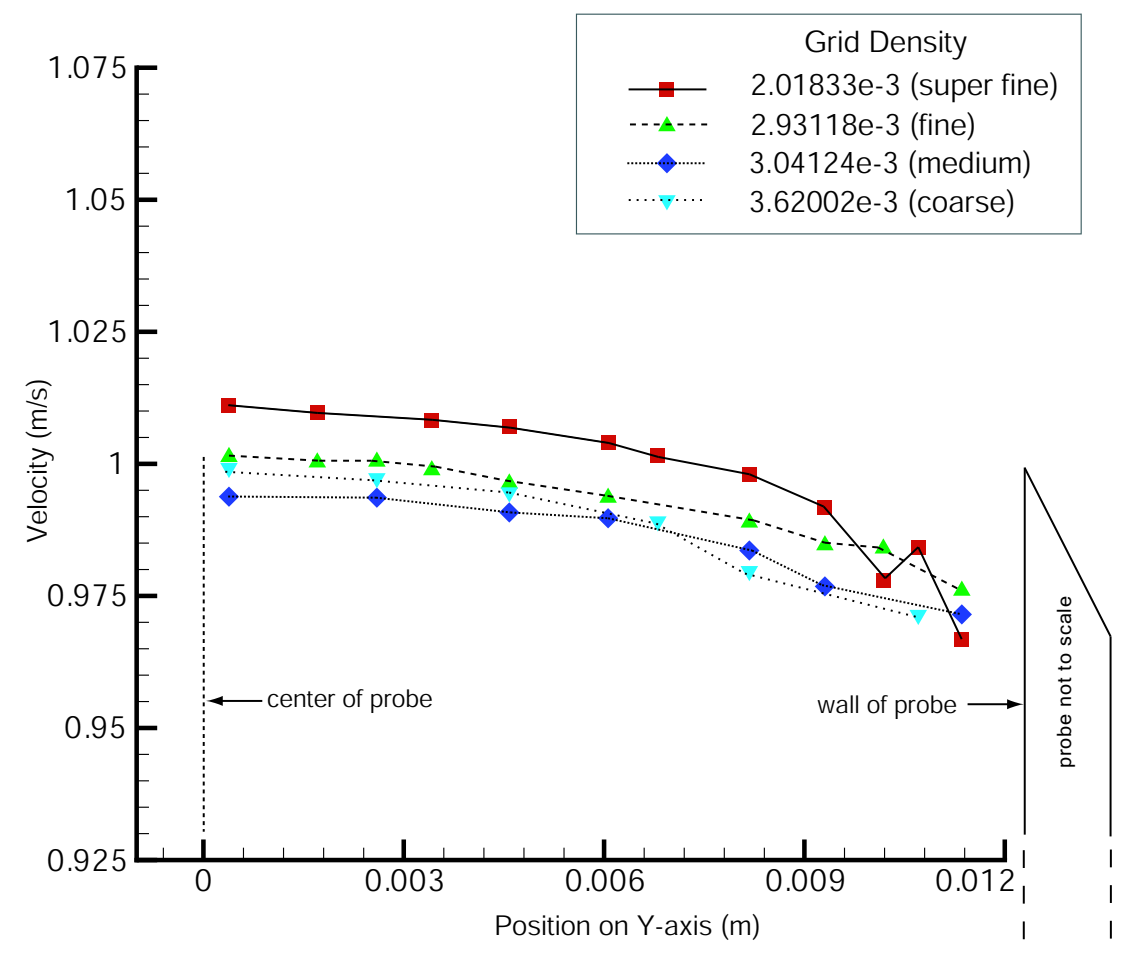

Figure 4.2: Velocity values of four grid densities across inlet of isokinetic sampler for 1.0 $\mathrm{m} / \mathrm{s}$ freestream and sampler inlet velocities. 
Influence from different orders of numerical method (1st- and 2nd-order, minimum) was evaluated by plotting the percent relative differences between calculations with the two different orders of numerical method. Data were plotted for results from the "fine" grid density simulations of the GSP and probe samplers at 0-, 90-, and 180-degree orientations to freestream at center and near-wall locations (see Figure 4.3 of GSP for example of analysis locations). The qualitative plots (see Figures 4.4 and 4.5) suggest there is little difference between orders for values taken from the center of the region, but that there is substantial difference at the walls.

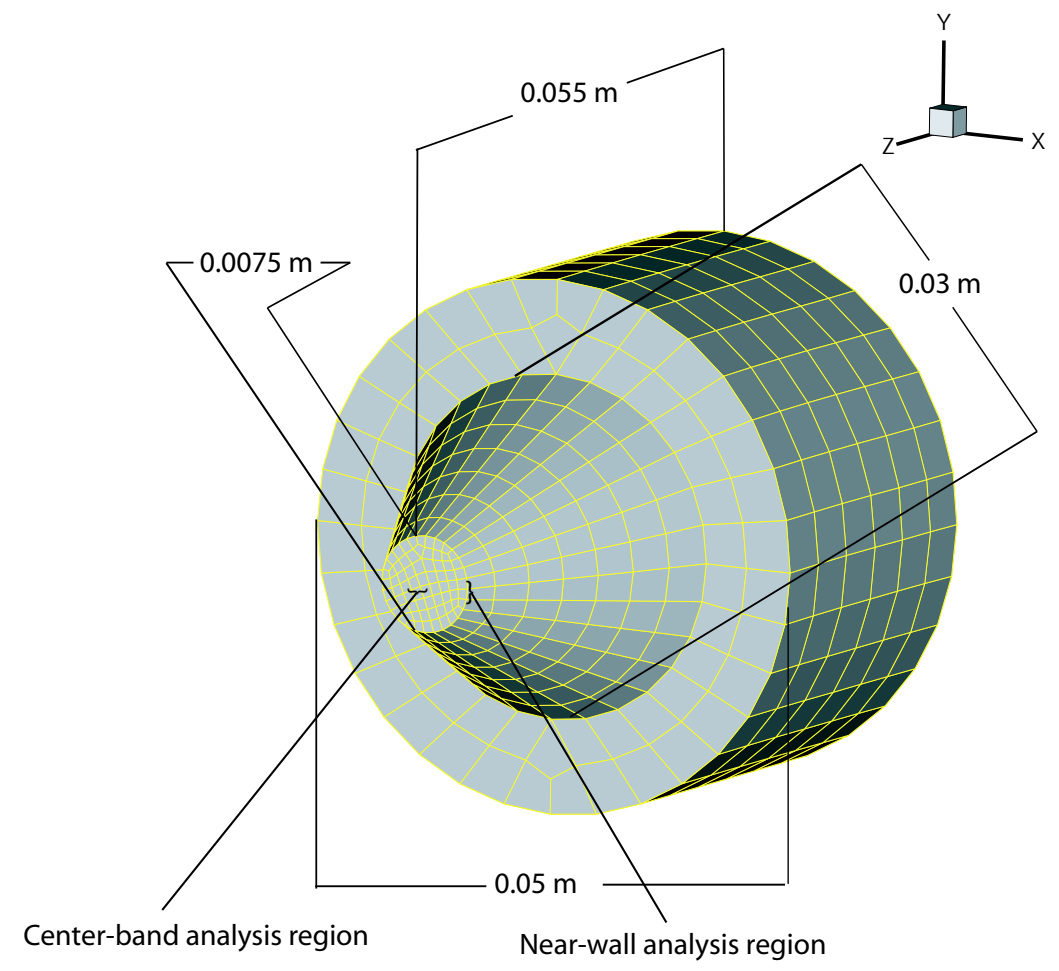

Figure 4.3: Center-band and near-wall analysis locations for GSP sampler. The reference probe analyses were done for similar spatial locations. 
GSP Sampler
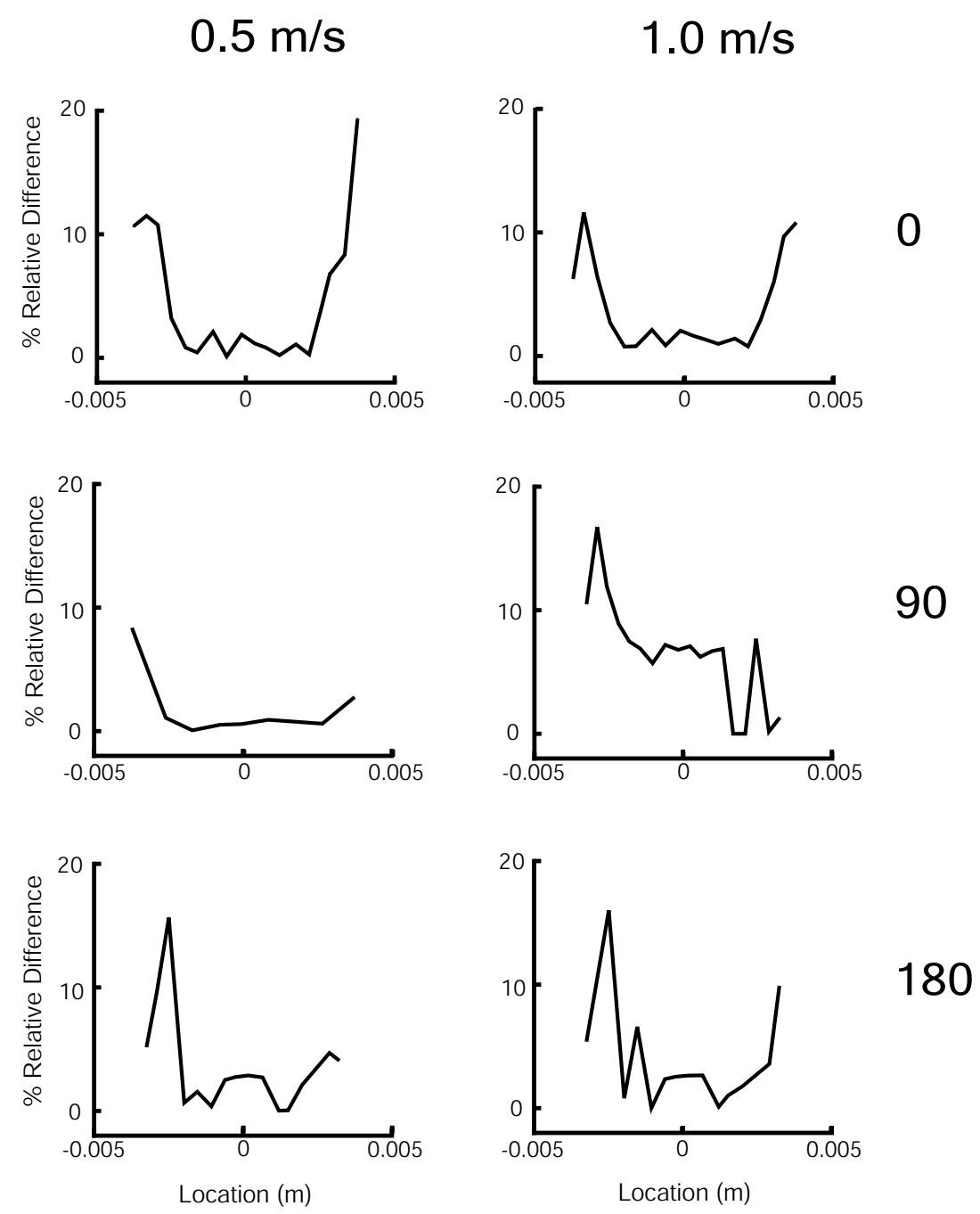

Figure 4.4: Percent relative differences between 1st- and 2nd-order CFD solvers for simulations of the GSP sampler for 0.5 and $1.0 \mathrm{~m} / \mathrm{s}$ freestream windspeeds at $0-$, 90-, and 180-degree orientations. 


\section{Reference Probe}

$0.5 \mathrm{~m} / \mathrm{s}$
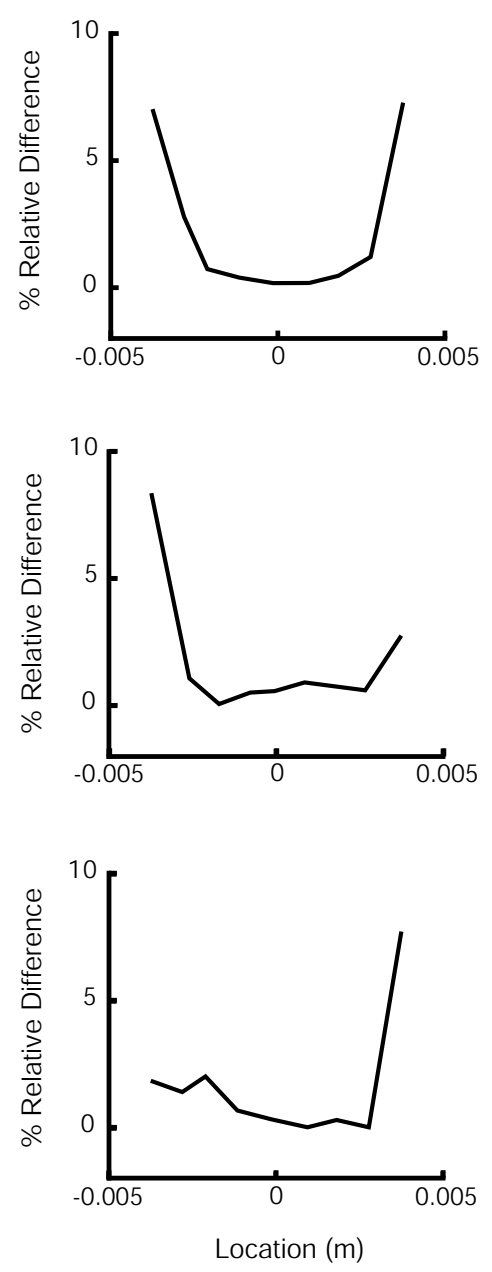

$1.0 \mathrm{~m} / \mathrm{s}$
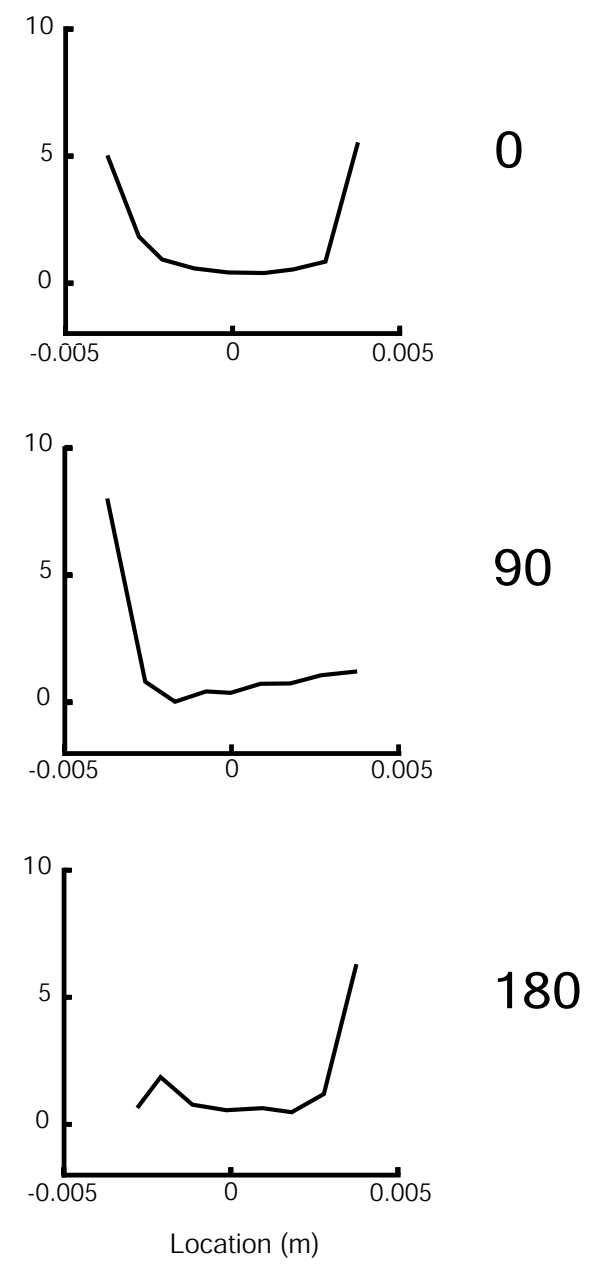

Figure 4.5: Percent relative differences between 1st- and 2nd-order CFD solvers for simulations of the reference probe for 0.5 and $1.0 \mathrm{~m} / \mathrm{s}$ freestream windspeeds at 0-, 90-, and 180-degree orientations. 


\section{Parameter Space Analysis}

The second method by which the parameter influences were evaluated was through use of a parameter space analysis method. This method is based on a non-statistical application of principles of factorial analysis. Parameters including grid, order of numerical method, and analysis location were compared at two levels. The "space," between them, i.e. the

average $\delta$ was determined by summing the differences between the low and high levels and dividing by the number of comparisons.

Comparisons were made for two-grid combinations with results from coarse, medium, fine, and superfine grids at both orders of solver and for both windspeeds. Location comparisons were made from results taken from the center of the sampler inlets (banded and point data) and at the walls of the samplers (again, banded and point data). All combinations were analyzed, however, only space values that had a $10 \%$ or larger deviation from the ideal values ( 0.5 or 1.0) are shown in Table 4.3 (see Appendix for all data).

Table 4.3 is broken into two sections. The top portion, consisting of only results from the probe sampler, is for freestream velocities of $0.5 \mathrm{~m} / \mathrm{s}$. The lower portion, consisting of both probe and GSP results, is for freestream velocities of 0.5 and $1.0 \mathrm{~m} / \mathrm{s}$. Thus, for the top portion, values that are $0.05 \mathrm{~m} / \mathrm{s}$ and greater are in the $10 \%$ cutoff region, and for the bottom portion, values $0.1 \mathrm{~m} / \mathrm{s}$ and larger are in the cutoff region. In the table, it can be seen that the observation location (spread) has the largest average deltas. By looking at the two-location comparisons, it is apparent that the near-wall (WN) and wall-edge (WE) 
Table 4.3: Values determined from parameter space analysis to yield $10 \%$ or greater influence on desired results are shown in bold.

\begin{tabular}{|c|c|c|c|c|c|c|}
\hline Sampler & $\begin{array}{l}\text { Wind } \\
\text { Speed }\end{array}$ & $\begin{array}{c}\text { Two-Grid } \\
\text { Comp. }\end{array}$ & $\begin{array}{l}\text { Two- } \\
\text { Location } \\
\text { Comp. }\end{array}$ & $\begin{array}{l}\text { Avg. Delta } \\
\text { due to } \\
\text { Grid } \\
(\mathrm{m} / \mathrm{s})\end{array}$ & $\begin{array}{l}\text { Avg. Delta } \\
\text { due to } \\
\text { Order } \\
(\mathrm{m} / \mathrm{s})\end{array}$ & $\begin{array}{l}\text { Avg. Delta } \\
\text { due to } \\
\text { Spread } \\
(\mathrm{m} / \mathrm{s})\end{array}$ \\
\hline Probe & 0.5 & Med, Sup & WN, WE & -0.05224 & -0.006118 & -0.07672 \\
\hline Probe & 0.5 & Fin, Sup & WN, WE & -0.02225 & -0.0088159 & -0.09295 \\
\hline Probe & 0.5 & Med, Sup & $\mathrm{CB}, \mathrm{WE}$ & -0.038255 & -0.008232 & -0.10511 \\
\hline Probe & 0.5 & Fin, Sup & $\mathrm{CB}, \mathrm{WE}$ & -0.017407 & -0.012712 & -0.130601 \\
\hline Probe & 1.0 & Fin, Sup & WN, WE & -0.063286 & -0.02147 & -0.118312 \\
\hline Probe & 1.0 & Med, Sup & $\mathrm{CB}, \mathrm{WE}$ & -0.052210 & -0.019425 & -0.134302 \\
\hline Probe & 1.0 & Fin, Sup & $\mathrm{CB}, \mathrm{WE}$ & -0.030947 & -0.026591 & -0.162006 \\
\hline GSP & 0.5 & Crs, Sup & WN, WE & 0.053560 & 0.021395 & -0.224005 \\
\hline GSP & 0.5 & Med, Sup & WN, WE & 0.0103025 & -0.081303 & -0.153488 \\
\hline GSP & 0.5 & Fin, Sup & WN, WE & 0.0414425 & 0.018475 & -0.283675 \\
\hline GSP & 0.5 & Med, Sup & $\mathrm{CB}, \mathrm{WE}$ & -0.033602 & -0.135593 & 0.0732193 \\
\hline GSP & 0.5 & Crs, Sup & $\mathrm{CB}, \mathrm{WN}$ & 0.0091545 & 0.088936 & 0.229158 \\
\hline GSP & 0.5 & Med, Sup & $\mathrm{CB}, \mathrm{WN}$ & 0.1537645 & 0.187043 & 0.106906 \\
\hline GSP & 0.5 & Fin, Sup & $\mathrm{CB}, \mathrm{WN}$ & 0.0034945 & 0.083846 & 0.265883 \\
\hline GSP & 1.0 & Crs, Sup & WN, WE & 0.100556 & 0.0558038 & -0.091769 \\
\hline
\end{tabular}

locations show the greatest differences. From this finding, it can be said that analyses using results from different locations in the computational domain will not lead to the same conclusions. Therefore the source of the data must be tracked when comparisons are made. Another telling finding from this table is that the grid and the order, by themselves, have few cases where their values exceed the $10 \%$ cutoff point. However, they still do need further examination, and this is addressed in the next section.

\section{Grid Convergence Studies}

Grid convergence studies were conducted based on the Grid Convergence Index (GCI) method by Roache (1998). This method allows a measure of effect of refining or coars- 
ening on the solutions of a simulation. It is done to determine if grids yield independent solutions for a particular class of flows and the result is a normalized relative percent uncertainty. The method is intended for application on structured cartesian grids that are refined in one direction at a time. However, Roache makes the claim that the method can be applied to unstructured and non-cartesian grids that are refined in more than one direction simultaneously, which is often the case for engineering and industrial applications. When grid convergence studies are applied to non-ideal cases, Roache recommends a "factor of safety," $F_{s}$, that is conservative. In this work, the recommended values of 1.25 and 3 (Roache's conservative value) were used, in addition to the case of no safety factor. In this application, the uncertainty is determined from no fewer than three grids and for two theoretical orders of the hybrid numerical method used in FLUENT. Grid sizes used in the calculation were the total number of nodes in the computational domain, thus, $r=$ (More Dense Grid)/(Less Dense Grid), where the densities, $h$, can be determined from equation 3.31 .

For the orders of the numerical method, FLUENT does not have a true 1st-order solver, therefore the apparent order of a 1 st-order method is actually $1<P<2$. This is a result of the advection terms being solved in a second-order discretization, while upwinding is solved in first order. Even so, if it is assumed that use of $P=1$ for $1<P<2$ is conservative, then comparisons can be made between the two theoretical applications of numerical solver, i.e. $P=1$ and $P=2$.

Figures 4.6 through 4.9 show the convergence of uncertainty due to grids from coarsest to 
finest for simulations of the probe and GSP samplers for the parameter levels evaluated. The lines in the plots represent the percent relative difference and grid convergence uncertainty at a safety factor of $1,1.25$, and 3 . With exception of the percent relative error, which is not normalized by the grid scaling, the convergence behavior is poor for the first-order solver, but good for the second-order solver. In addition, grid convergence evaluations using the second-order solver shows a decrease in uncertainty to generally lower levels than that of the first-order solver. 


\section{GSP Sampler Grid Convergence $0.5 \mathrm{~m} / \mathrm{s}$ Freestream}

$-=-1, \quad X 1$

$X 1.25$

-

Relative Difference

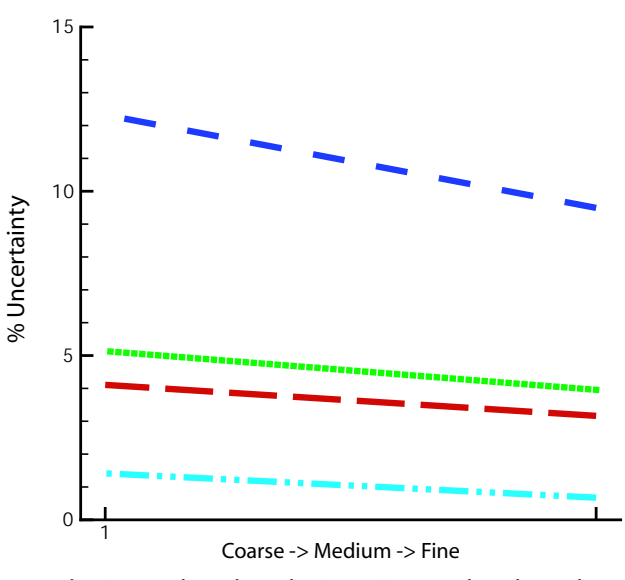

b:Center-band analysis region, 2nd-order solver.

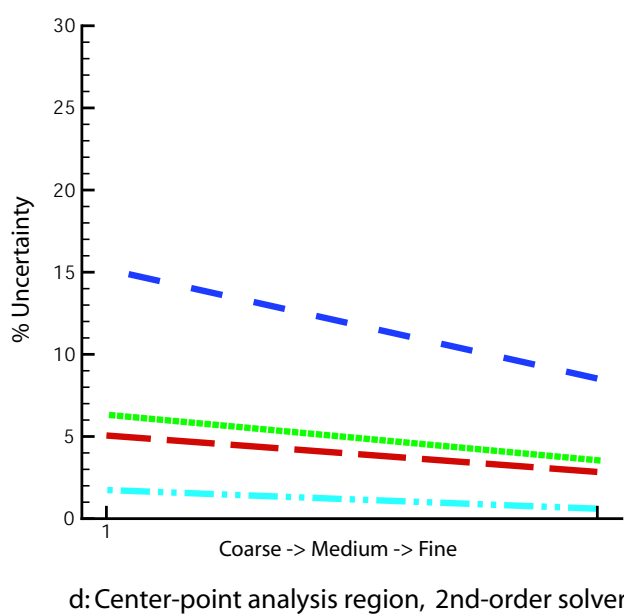

$\mathrm{d}$ :Center-point analysis region, 2 nd-order solver.

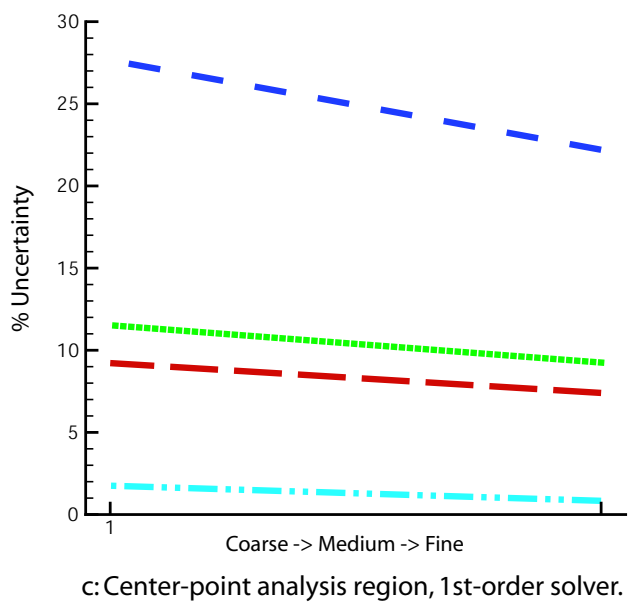

Figure 4.6: Plotted uncertainties determined from grid convergence studies on three grid densities of simulation results of GSP sampler at $0.5 \mathrm{~m} / \mathrm{s}$ and 0 -degree orientation. 


\section{GSP Sampler Grid Convergence $1.0 \mathrm{~m} / \mathrm{s}$ Freestream}

\begin{tabular}{|c|c|}
\hline 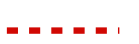 & $\mathrm{X} 1$ \\
\hline 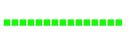 & $X 1.25$ \\
\hline 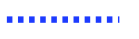 & X3 \\
\hline 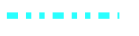 & Relative Diffe \\
\hline
\end{tabular}

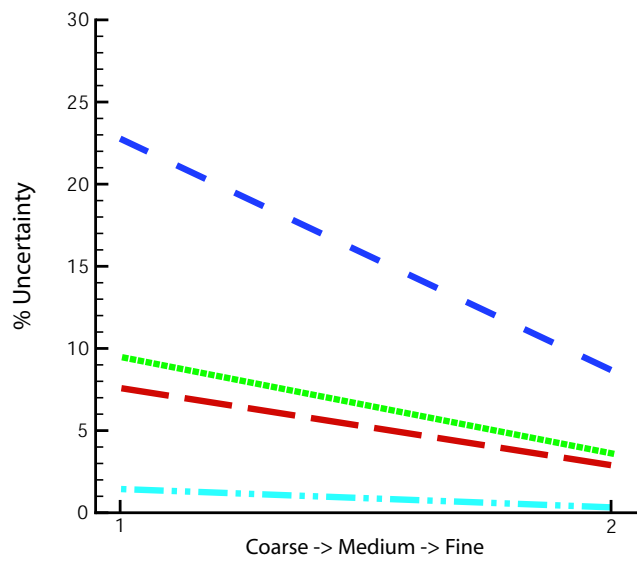

a:Center-band analysis region, 1 st-order solver.

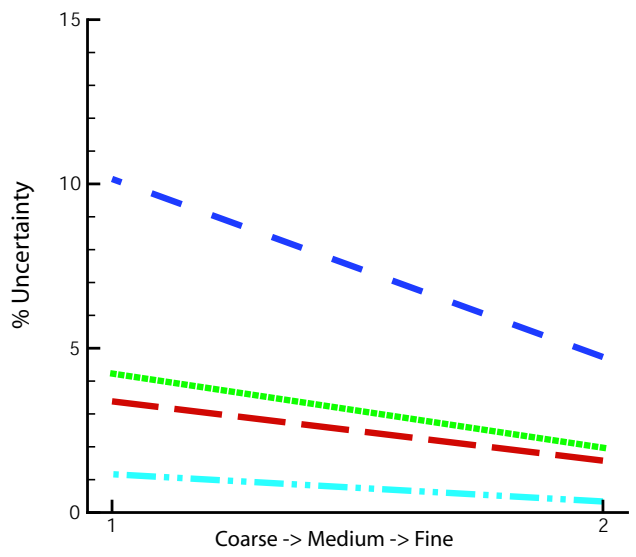

b: Center-band analysis region, 2nd-order solver.
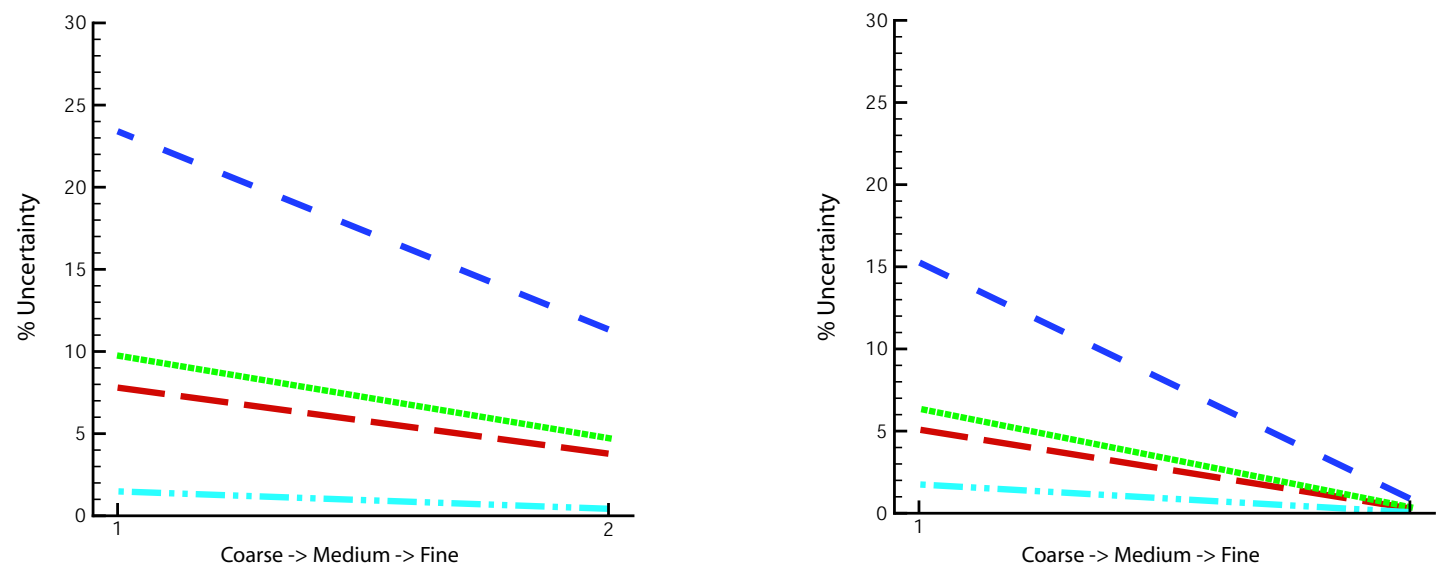

c: Center-point analysis region, 1 st-order solver.

Figure 4.7: Plotted uncertainties determined from grid convergence studies on three grid densities of simulation results of GSP sampler at $1.0 \mathrm{~m} / \mathrm{s}$ and 0 -degree orientation. 


\section{Probe Sampler Grid Convergence $0.5 \mathrm{~m} / \mathrm{s}$ Freestream}

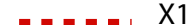

X1.25

mand X3

Relative Difference

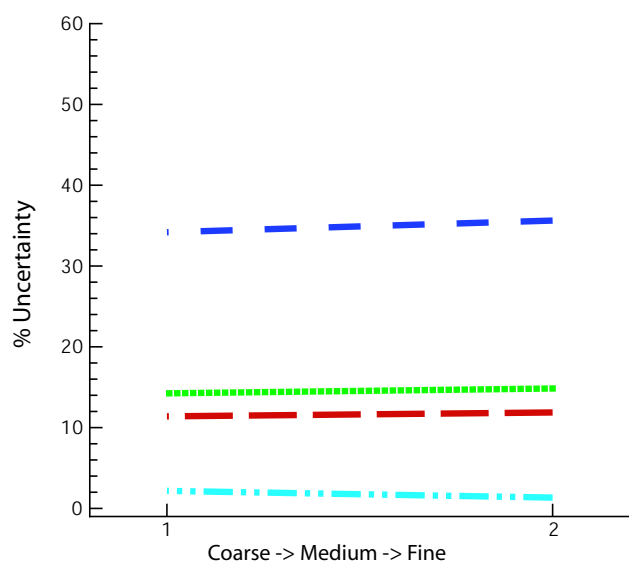

a: Center-band analysis region, 1st-order solver.

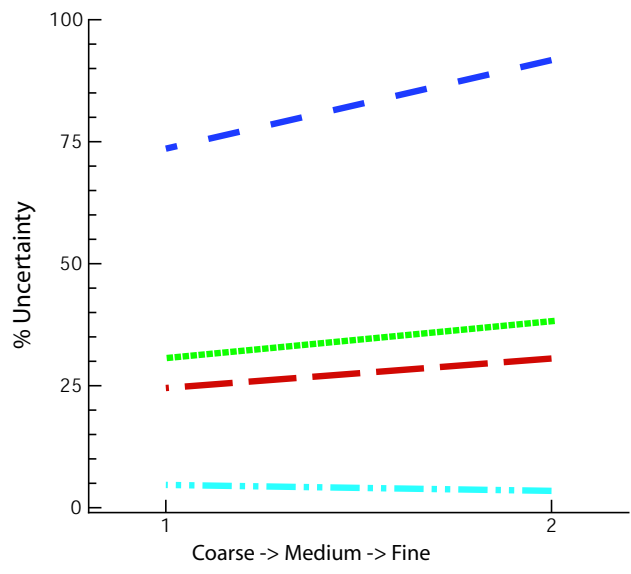

c: Near-wall analysis region, 1st-order solver.

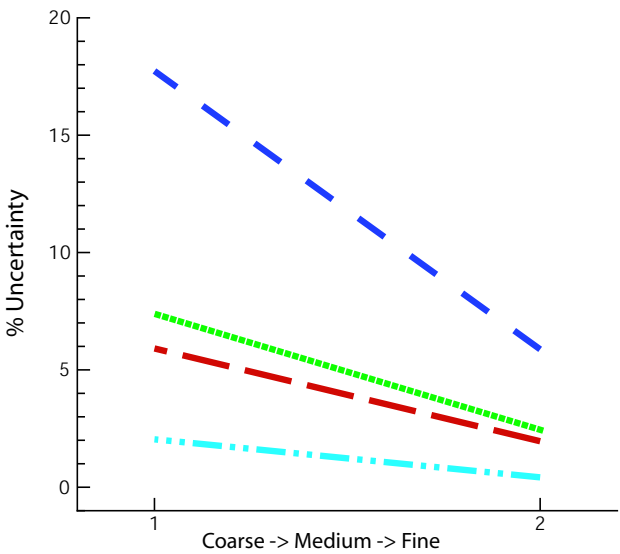

b:Center-band analysis region, 2nd-order solver.

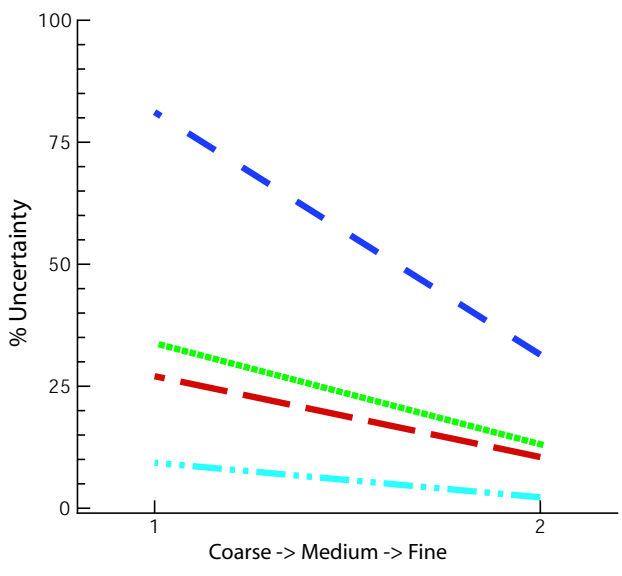

d: Near-wall analysis region, 2nd-order solver.

Figure 4.8: Plotted uncertainties determined from grid convergence studies on three grid densities of simulation results of GSP sampler at $0.5 \mathrm{~m} / \mathrm{s}$ and 0 -degree orientation.

Click for List of Figures 


\section{Probe Sampler Grid Convergence $1.0 \mathrm{~m} / \mathrm{s}$ Freestream}

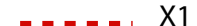

X1.25

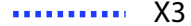

Relative Difference

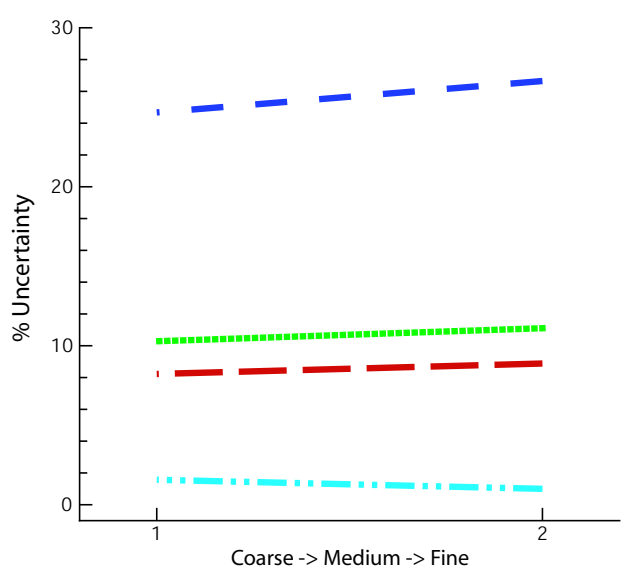

a: Center-band analysis region, 1 st-order solver.

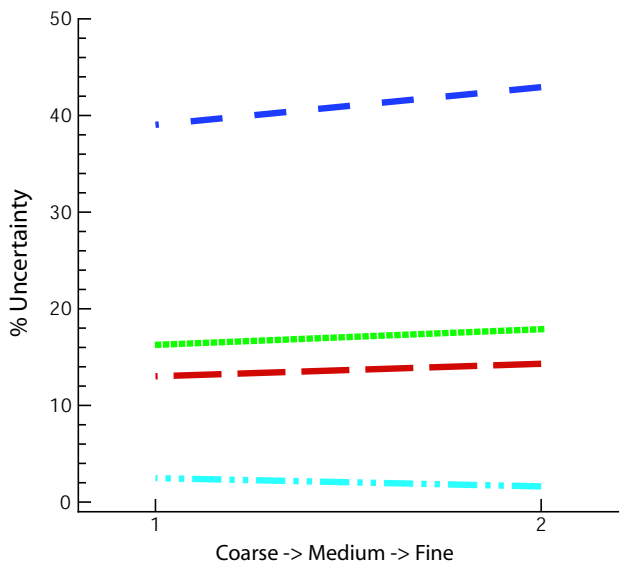

c: Near-wall analysis region, 1st-order solver.

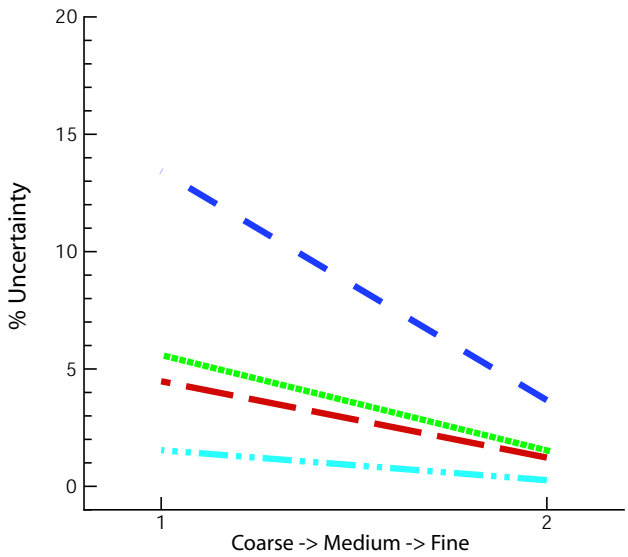

b:Center-band analysis region, 2nd-order solver.

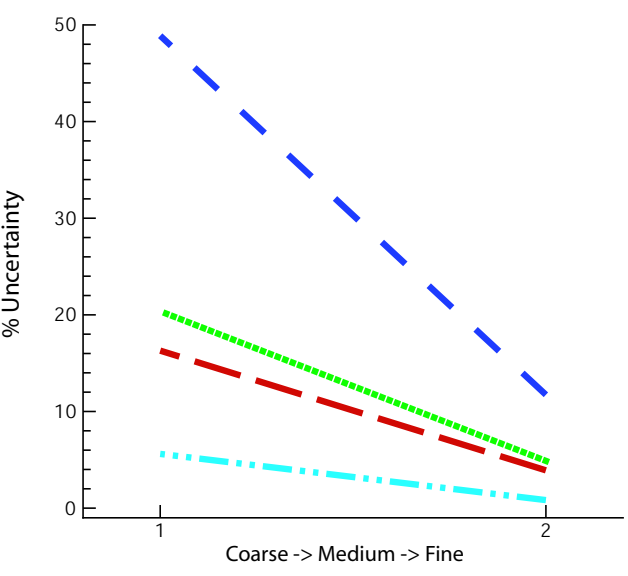

d: Near-wall analysis region, 2nd-order solver.

Figure 4.9: Plotted uncertainties determined from grid convergence studies on three grid densities of simulation results of GSP sampler at $1.0 \mathrm{~m} / \mathrm{s}$ and 0 -degree orientation. 


\section{Wall-Mesh Considerations}

Analyses of simulations described in the previous sections have shown that the grids are independent. However, it is clear there are differences between solvers (1st- and 2nd-order) at the walls for the same grids. Thus, to ensure results are accurate at the walls, simulations were checked for consistency with requirements for use of the RNG k-e turbulence model.

Mesh elements within the computational domain were evaluated for adherence to RNG k-e requirements at two primary regions in the domain: near-wall and near-inlet. Near-wall grid element sizes were chosen based on two requirements: (1) there must be at least 10 cells between a cell with the value of $R e_{T u r b}=200$ and the wall, and (2) $y_{\text {wall }}^{+}$must be less than 5, preferably less than 2, at a point just beside the wall (Fluent, Inc., 2003).

Tetrahedral cell grids were refined in a step-wise process and with cell adaption, a method used to split existing mesh cells into smaller cells, in order to yield a mesh that conformed to the above requirements (see Figures 4.10 and 4.11). For freestream velocity conditions of $0.5 \mathrm{~m} / \mathrm{s}$, mesh refinement resulted in turbulent Reynolds number of 31.7 at a point ten cells outside of the sampler's wall, and 14.5 at 10 cells within the sampler (Figure 4.12). For freestream velocity of $1.0 \mathrm{~m} / \mathrm{s}$, turbulent Reynolds numbers were 33.7 and 30.1 , respectively (Figure 4.13). The values of $y_{\text {wall }}^{+}$were 2.7 and 4.01 for the 0.5 and $1.0 \mathrm{~m} / \mathrm{s}$ freestream flows. This suggests the near-wall mesh was well resolved for this class of flows using the particular solver and turbulence models applied. 


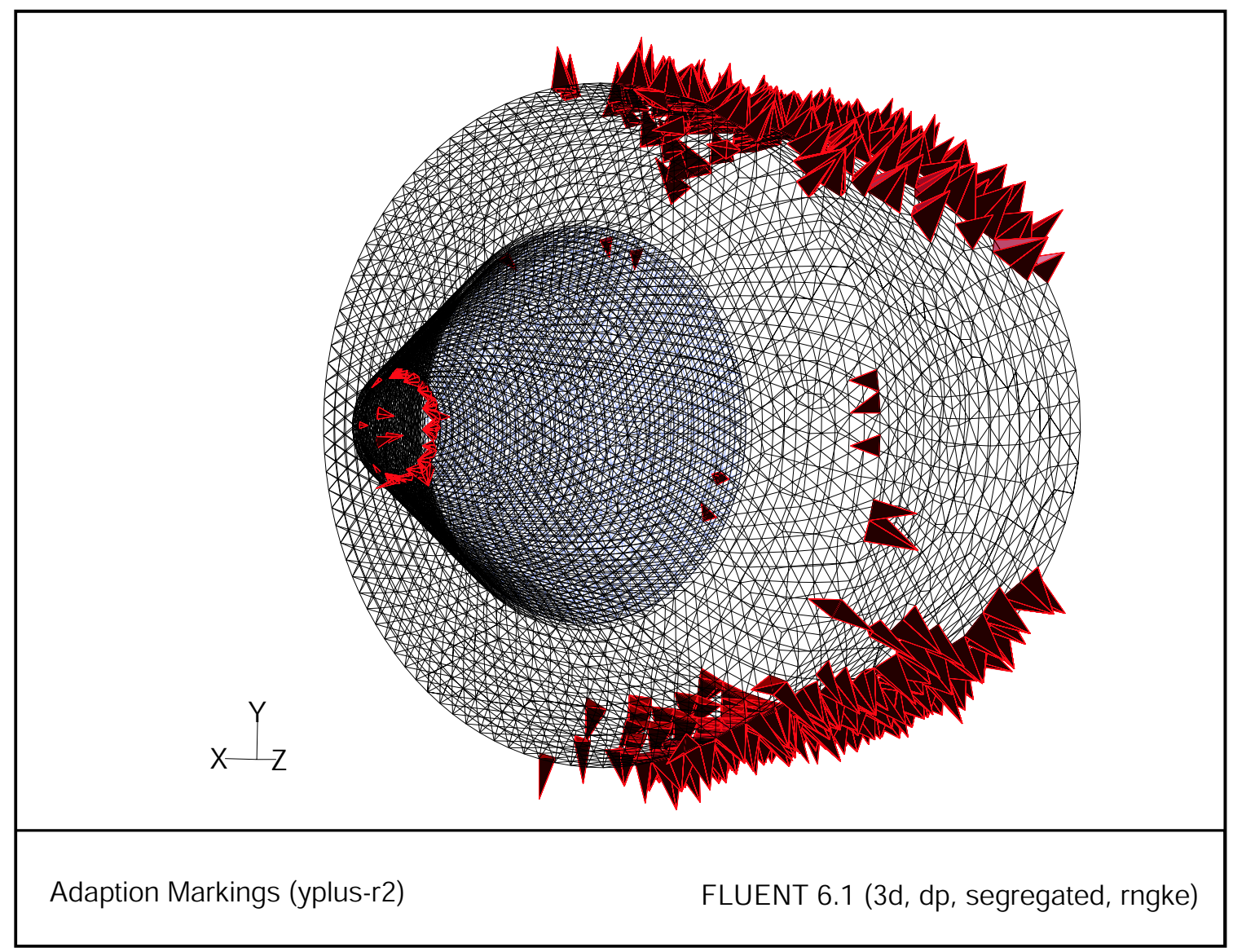

Figure 4.10: Noncomforming grid elements on inside and outside surfaces of GSP sampler for turbulent $y^{+}$values before adaption. See Figure 4.11 for detailed mesh adaption inside the inlet. 


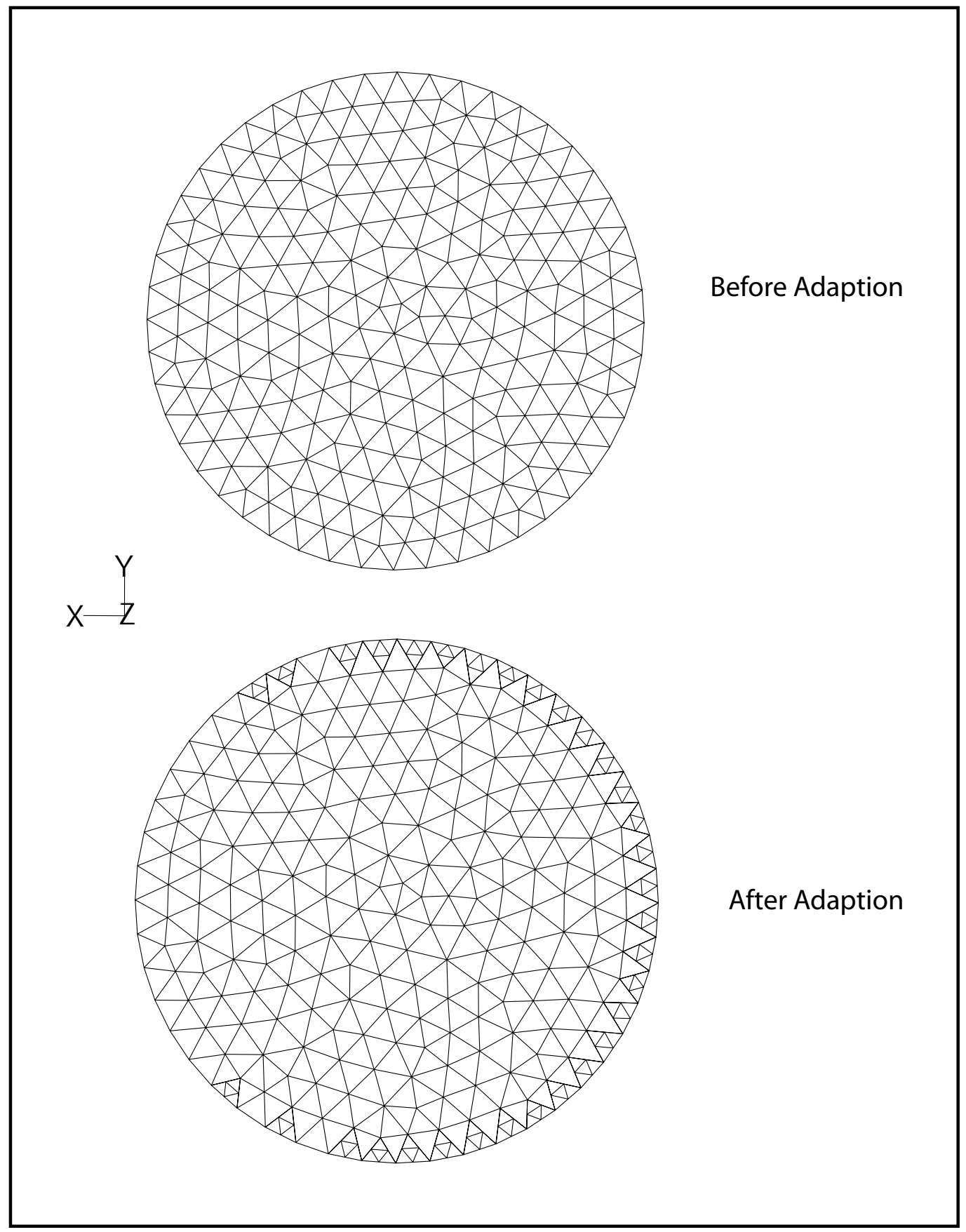

Figure 4.11: Before and after adaption for turbulent $y^{+}$values inside the inlet of the GSP sampler. There is greater adaption on the right side because this is the upstream side of the sampler and more turbulence is present here. 


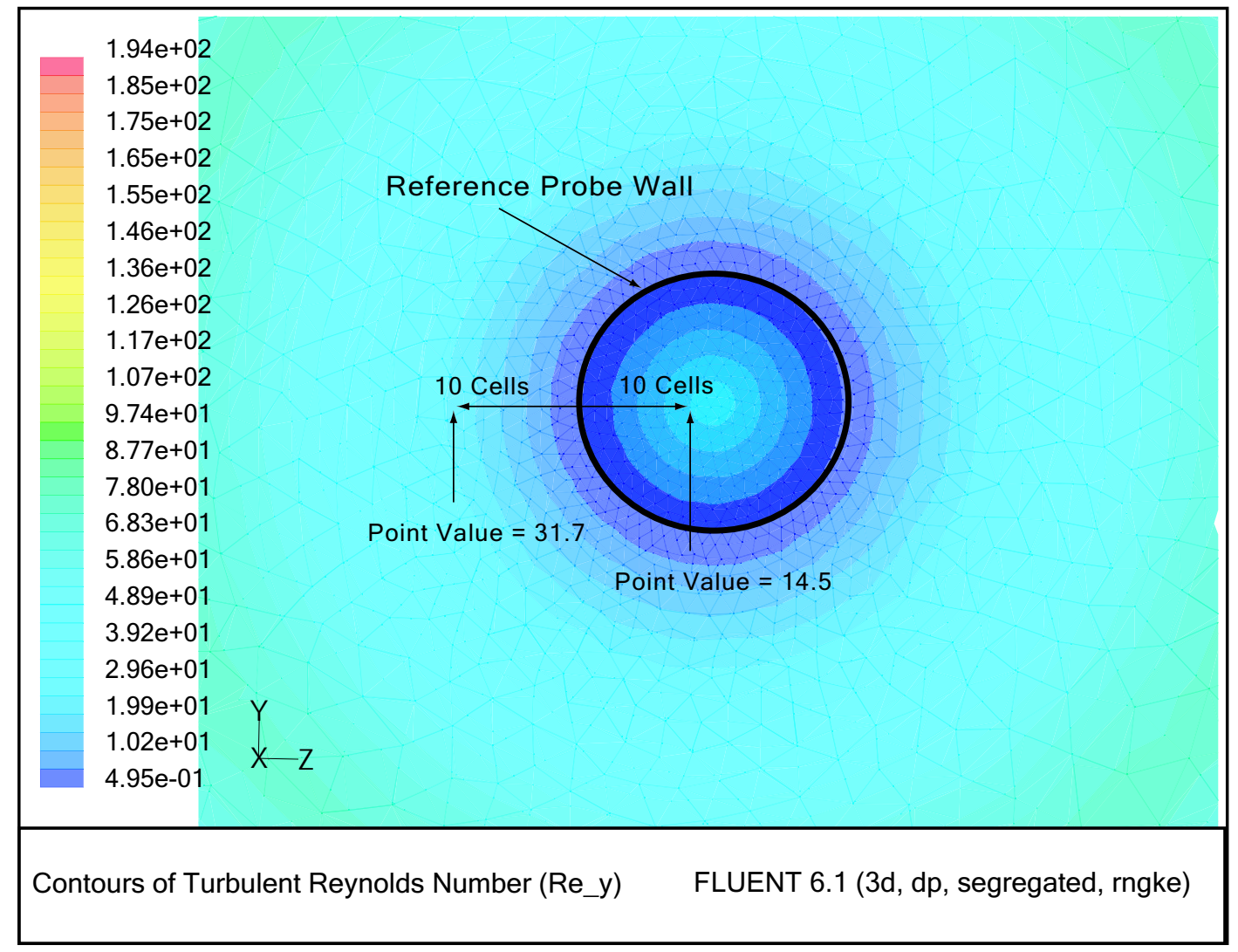

Figure 4.12: Contour plot of turbulent reynolds number values for probe reference sampler in freestream flow of $0.5 \mathrm{~m} / \mathrm{s}$. View is looking downstream toward inlet of sampler. 


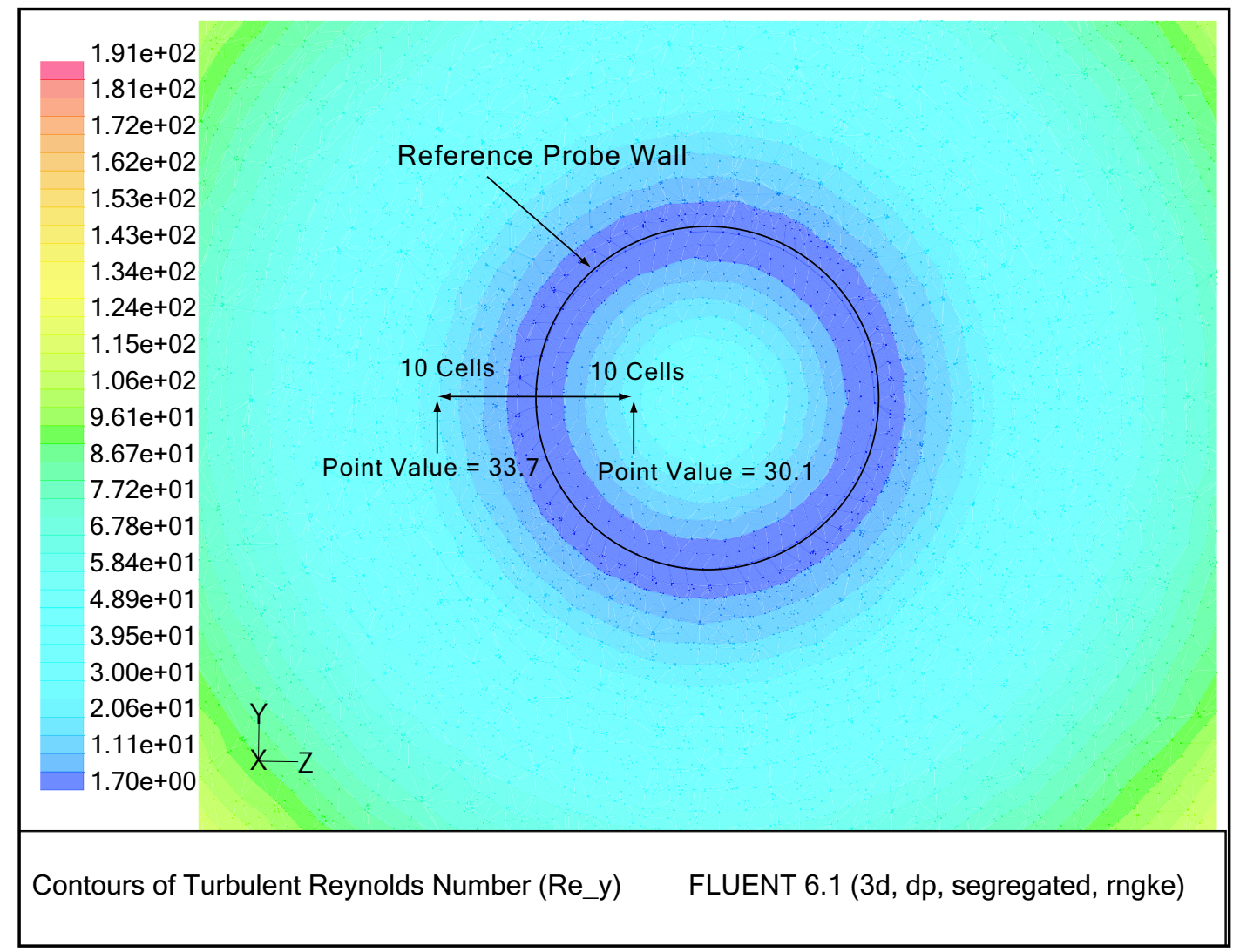

Figure 4.13: Contour plot of turbulent reynolds number values for probe reference sampler in freestream flow of $1.0 \mathrm{~m} / \mathrm{s}$. View is looking downstream toward inlet of sampler. 


\subsubsection{CFD Simulations Summary}

A number of important parameters were identified that are critical for correct and accurate simulations of the aerosol samplers investigated in this work. They are as follows:

Freestream Velocities: 0.5 \& 1 m/s

Orientations: 0-, 90-, and 180-degrees

Grid Type: Tetrahedral

Grid Size: $0.0010 \mathrm{~m}$ at inlet

CFD Solver: 2nd-order

Turbulence Model: RNG $k-\epsilon$ with enhanced wall function and differential viscosity Turbulence Intensity: $5 \%$ at domain inflow; $3 \%$ outflow

Length Scale: $0.01 \mathrm{~m}$ at inflow; equal to minimum grid size at outflow

Number of Iterations: Achieve 6-orders-of-magnitude decrease from initial conditions Analysis Regions: Center-band and near-wall 


\subsection{Particle Trajectory Calculations of Samplers}

Particle trajectories were calculated in a Lagrangian reference frame using the FLUENT Discrete Phase Model and based on the optimized parameters and parameter levels of CFD simulations found in the previous sections. In a CFD simulation, the concentration of aerosols (particle tracks) must be calibrated in order that the thin-walled, sharp-edged probe sees the same number of particles as does an arbitrarily thin disk (referred to as an "ideal sampling face," see Figure 4.14) in the undisturbed freestream flow. When this occurs, it is confirmation of sampling the true airborne concentration, which is sampling with unity.

The particles were spherically shaped and had a density equal to that of anthracite $(1,550$ $\mathrm{kg} / \mathrm{m}^{3}$ ). They were distributed in monodisperse groups of $1,5,10,20,30,40,50,60$, 70, 80, 90, and $100 \mu m$ in aerodynamic diameter. Release faces and concentrations require calibration so that isokinetic, isoaxial probe samplers resulted in aspiration efficiencies of unity. This was achieved by releasing particles from a variety of faces and locations throughout the computational domain until there was equality between the freestream concentration and inlet concentration. Release lines could be rotated in 360 degrees in order to yield dense particle distributions (A through F, Figure 4.14).

Approximately 100,000 particles were released from ten regions including five sources upstream from the aerosol aspiration faces and one source located above the aerosol aspiration faces. Particles released from faces perpendicular to freestream flow were given an initial velocity of $-0.25 \mathrm{~m} / \mathrm{s}$. These particles initially travelled for a short distance up- 


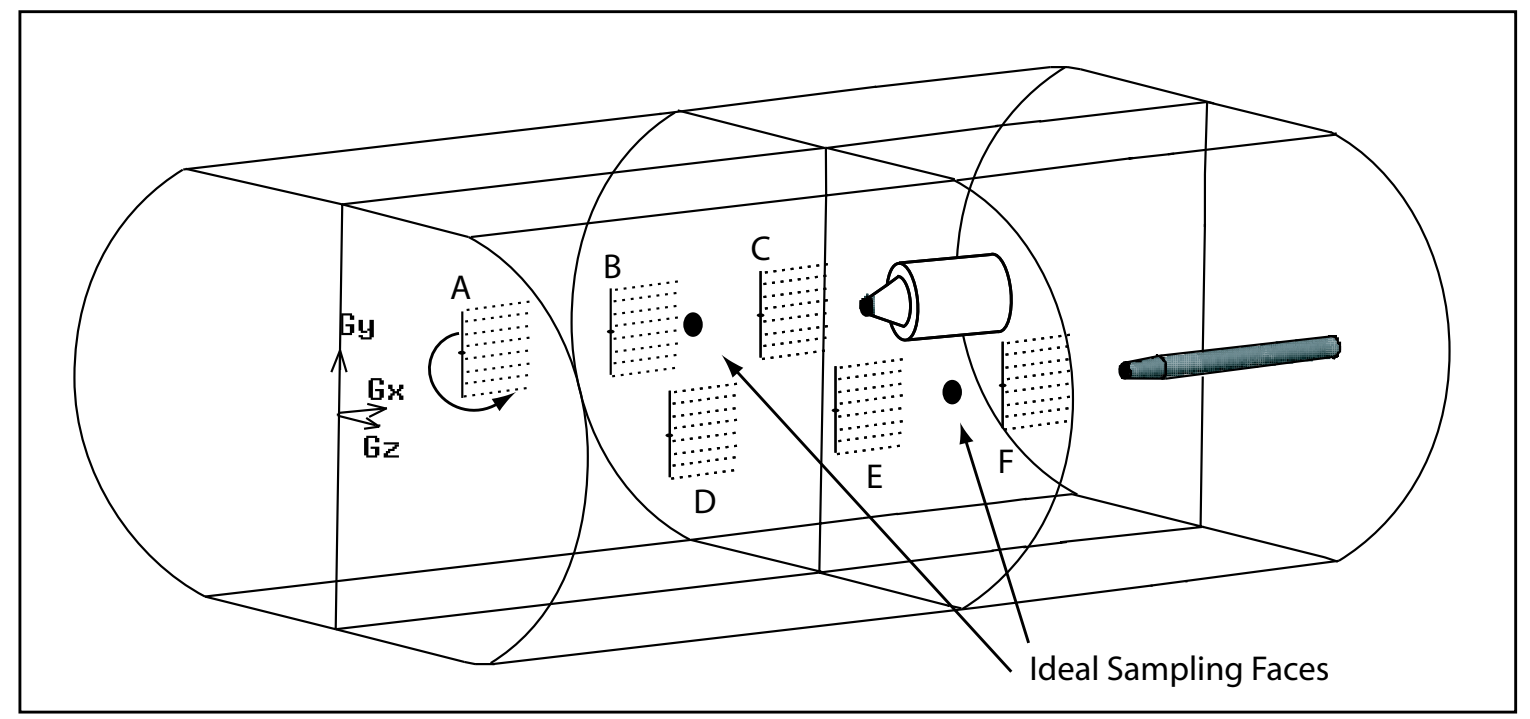

Figure 4.14: Wireframe diagram of ideal sampling faces, conical inlet section of GSP sampler, probe reference sampler, and locations of aerosol release lines.

stream until the magnitude and direction of the flow in the computational domain, together with the force of gravity, overcame the upstream momentum and entrained them into the downstream flow. Particles released from the top face were initially given an upward initial velocity of $0.1 \mathrm{~m} / \mathrm{s}$, and these particles, too, were subsequently affected by downstream flow and gravity and then entrained into the freestream flow.

A trial-and-error approach was used to determine the best combination of release faces and concentrations in order to achieve unity between the concentrations seen by the aerosol aspiration faces. For aerosol aspiration in freestream flow, i.e. determination of the true concentration in undisturbed flow, the number of particles travelling across the face were counted and this number was used as the value for concentration. For the sharp-edged 
probe, aspiration was defined as the number of particles exiting from the outflow of the probe, which was located two inlet diameters downstream from the probe inlet. Wall losses were also accounted for, and were found to be less than $1 \%$ for particle sizes up to $40 \mu \mathrm{m}$. This is expected to be due to the slight acceleration of the fluid within the probe, which leads to a concentrating effect of particles into the center flow region of the tube (Rao et. $a l, 1993)$. For particle sizes up to $100 \mu m$, wall losses were significant, which also is an expected result.

Near-unity agreement was found for particle sizes up to $40 \mu m$ (Figure 4.15) in freestream windspeeds of 0.5 and $1.0 \mathrm{~m} / \mathrm{s}$. For particle sizes between 50 and $100 \mu \mathrm{m}$, the release face locations and concentrations resulted in significant under-sampling depending on the combination of release faces, concentration, and particle size. Thus, the optimal condition of near-unity agreement was not achieved for particle sizes larger than $40 \mu \mathrm{m}$. However, the inhalability convention curve (based on experiment) shows transition at the $40 \mu \mathrm{m}$ level, therefore it is not unexpected that a transition would also be seen in simulation.

The finding of non-unity agreement above $40 \mu \mathrm{m}$ made determination of an accurate sampling efficiency very difficult. Collection of particle tracking information for both the sampler and for the ideal sampling face was attempted. This was done because the number of particles passing the ideal sampling face then continuing downstream to be aspirated by the sampler, i.e. the particle flux, is a function of the local flow conditions. Thus, it was hoped that by comparing the particle flux for the reference probe to the flux for the test sampler, a calculation of sampling efficiency for particle sizes larger than $40 \mu \mathrm{m}$ would be 
possible. Unfortunately this was not the case, and only a few accurate sampling efficiencies above $40 \mu m$ were possible. 


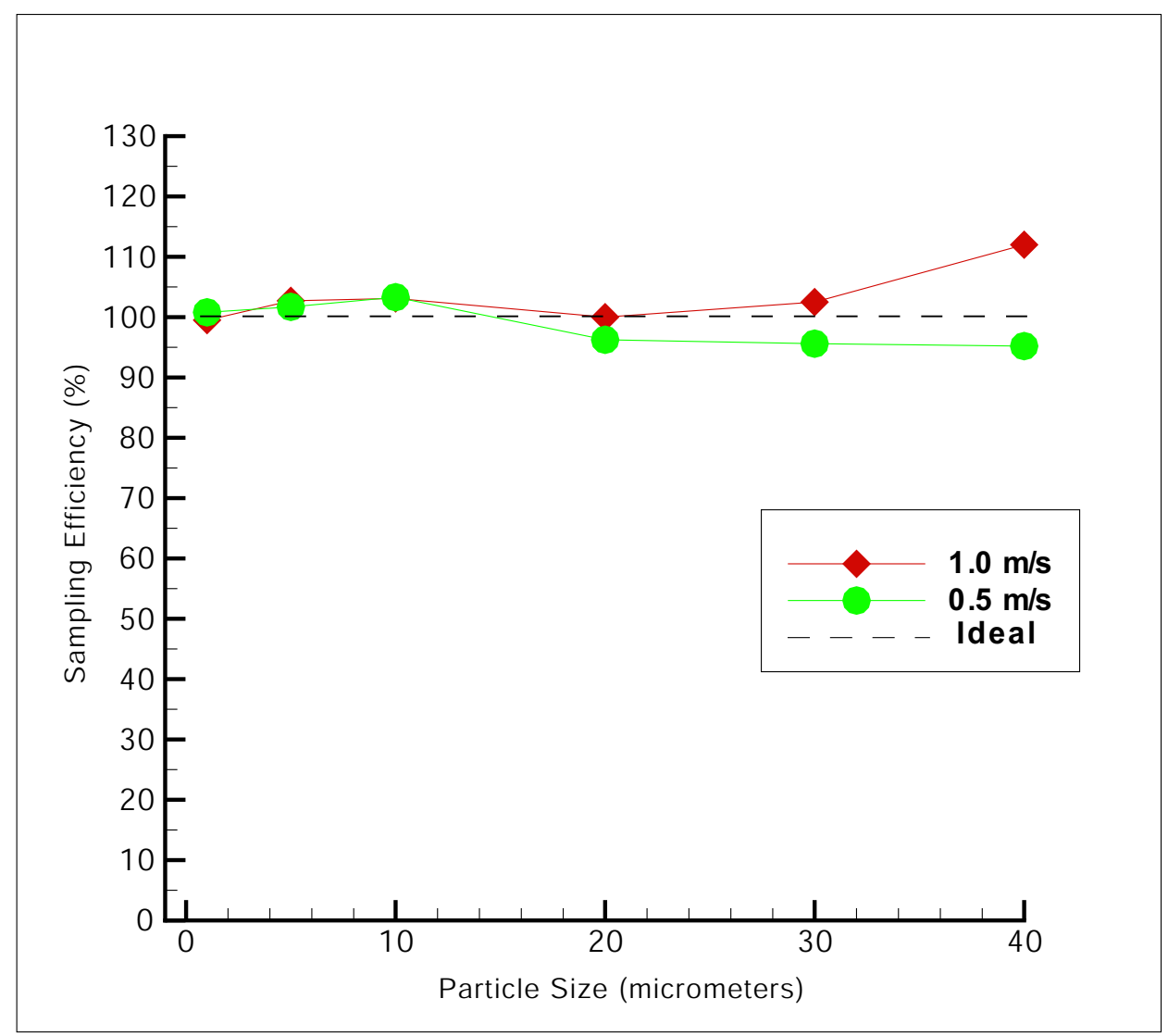

Figure 4.15: Sampling efficiency of sharp-edged probe sampler from FLUENT calculations at freestream velocities of 0.5 and $1.0 \mathrm{~m} / \mathrm{s}$. 


\subsubsection{GSP Personal Sampler}

Evaluation of the GSP personal sampler was based on the method used to calibrate the computational domain for sampling efficiency calculations of the ideal probe sampler. Grids were created for samplers free in the air and mounted on bluff bodies for 0-, 90-, and 180-degree orientations to the freestream flows having windspeeds of 0.5 and $1 \mathrm{~m} / \mathrm{s}$.

\section{GSP Free in the Air}

\section{Direction-Specific Results: $0.5 \mathrm{~m} / \mathrm{s}$ Freestream Windspeed}

The GSP personal sampler was evaluated in $0.5 \mathrm{~m} / \mathrm{s}$ freestream air by placing it in the same computational domain as the reference probe sampler (Figure 4.14). The samplers were situated in the domain so that their inlets were located at the same $\mathrm{x}$ distance downstream from the inflow face, and at equidistant $\mathrm{y}$ and $\mathrm{z}$ locations from the walls and centerline of the domain. Spatially the samplers were far enough apart so there was no effect from the presence of one on the other. Ideal reference faces were defined $0.1 \mathrm{~m}$ upstream of both the GSP and probe samplers, which is beyond where any distortion of flow occurs due to the presence of the samplers.

Particles were released from specific locations within the computational domain based on trajectory path parameters determined for the reference sampler. Sampler flux ratio, (test sampler flux)/(reference probe flux), was determined for each sampler evaluated. Particle flux ratio into the GSP sampler was determined for filter-only conditions and for total flux, 
which included filter collection + wall losses. The GSP is intended by its manufacturers to be used with filter-only collection. However, it was deemed useful in this work to collect wall-loss information in order to make complete comparisons with existing experimental data.

In Figure 4.16, the data plotted in the dashed line are the direction-specific calculated sampling efficiencies from CFD simulation of the GSP sampler at a 0-degree orientation to $0.5 \mathrm{~m} / \mathrm{s}$ freestream flow for "filter-only" sampling. Sampling efficiency data are shown for CFD simulations of particle sizes from 1 to $40 \mu \mathrm{m}$. Efficiencies were calculated for larger particle sizes as well. However, agreement with experiment -even with correction factors- was extremely poor. Furthermore, results from isokinetic sampling of the reference probe for particle sizes larger than $40 \mu m$ was much less than unity. Thus, it is difficult to normalize the sampling efficiencies to yield accurate results.

Also shown in Figure 4.16 are the sampling efficiencies measured in a wind tunnel by Li (1999) for particle sizes from 1.6 to $68 \mu \mathrm{m}$ in similar orientations and windspeeds. For this physical scenario, CFD simulation overpredicted experimental sampling efficiencies by nearly two times. Thus, a tuning correction factor of 0.7 was used to adjust the data down so agreement with experiment was reasonable. Corrected sampling efficiency is plotted in Figure 4.16 as a solid line.

Tuning of the simulation results was done with a constant correction factor determined from the average of the ratios of values from experiment divided by those obtained from simulation. The average tuning correction factor for the particle size range from 1 to 20 
$\mu m$ was used for all of the data in a particular set, i.e. in a particular scenario. Only one tune step was made for each data set, that is, one value was multiplied or one value was added to each individual result. Once this was done, no further adjustments were made to the simulation results so the tune step did not replace simulation as a modeling method.

Figure 4.16 also contains a table of percent relative disagreement (difference) values for comparisons between experimental results and corrected simulation results. Agreement is excellent for comparisons of sampling efficiencies at 1.6 to $21 \mu \mathrm{m}$. However, disagreement is significant at the $40-\mu m$ particle size.

Figure 4.17 shows data for "total" sampling efficiencies, which are for the sum of filter sampling and wall losses. Again, a tuning correction factor (0.56) was used to shift the data down so there was good agreement with the experimental values from Li. In both 4.16 and 4.17 there is a "bump" in the CFD simulation results for efficiency at the $30-\mu m$ particle size. Since the results of the sampling efficiency calculations are deterministic, it is usually expected that there would not be large deviations from the major trend of the curve. In this case there is, and there are several possible explanations for why this is so. In the percent relative disagreement table of Figure 4.17, it can be seen that simulation and experiment are close up to the 21- $\mu \mathrm{m}$ particle size. However, at the $40-\mu \mathrm{m}$ particle size the disagreement has increased.

Figures 4.16 and 4.17 both show excellent agreement for the smaller particle sizes. This is an expected result because these particles are more likely to follow the airstream and have minimal wall losses. However for the larger particle sizes, wall losses can be significant 
and lead to large discrepancies between simulation and experimental results.

Figure 4.18 shows plots of simulation and experimental results for filter-only sampling at an orientation of 90 degrees to a $0.5 \mathrm{~m} / \mathrm{s}$ freestream flow. In this orientation, grid refinement was very important due to the increase in near-wall turbulence prior to entry into the sampler. In the 0-degree orientation, this was not a major issue because the inlet was the point farthest upstream and thus the contribution to newly generated turbulence by the inlet had minimal effect on approaching particles. However at 90 degrees, there is a substantial effect of newly generated turbulence on approaching particles due to the fifty times increase in surface area prior to entering the sampler. Thus, grid adaption for values of $y_{\text {wall }}^{+}$ was conducted in order to ensure that the proper grid density was present at the surface. Nonconforming grid elements were identified (see Figures 4.10 and 4.11) and subdivided in order to achieve a greater density so there was adequate resolution to yield correct wall values.

In Figure 4.18, sampling efficiency trends were very consistent between corrected simulation and experimental results for filter-only sampling. However, the disagreement at 40- $\mu m$ was rather large, even though the absolute difference was small, only $13 \%$. A similar result is seen in Figure 4.19 for total sampling, where disagreement at $40-\mu m$ exceeds $90 \%$, but the absolute difference is less than $5 \%$.

At the 180-degree orientation for filter-only sampling in $0.5 \mathrm{~m} / \mathrm{s}$ freestream air (Figure 4.20), disagreement is large for all comparisons. The CFD simulation result at the 5- $\mu m$ particle size is suspect because it shows large deviation from the trend of the data either 
side of this value, i.e. $106 \%$ sampling efficiency at $1 \mu \mathrm{m}$ and $100 \%$ at $10 \mu \mathrm{m}$. It is believed that this discrepancy, and the overall poor agreement of this simulation, is due to the low collection flux of particles from upstream of the sampler to downstream. The ideal sampling faces, located upstream beyond the zone of flow distortion, and the isokinetic probe, also located away from the zone of flow distortion, both collect adequate numbers of particles: in the range of 2500 to 5000 . However, the filter of the GSP sampler sees only a tiny fraction of these particles after they are carried by the flow around the sampler and into its inlet: in the range of 14 to 500. When the wall losses are taken into consideration (Figure 4.21), some of the high disagreement between simulation and experiment was made up. Even so, overall agreement is still rather poor, and it very likely is because the domain is seeded with too few particles to yield accurate results. 


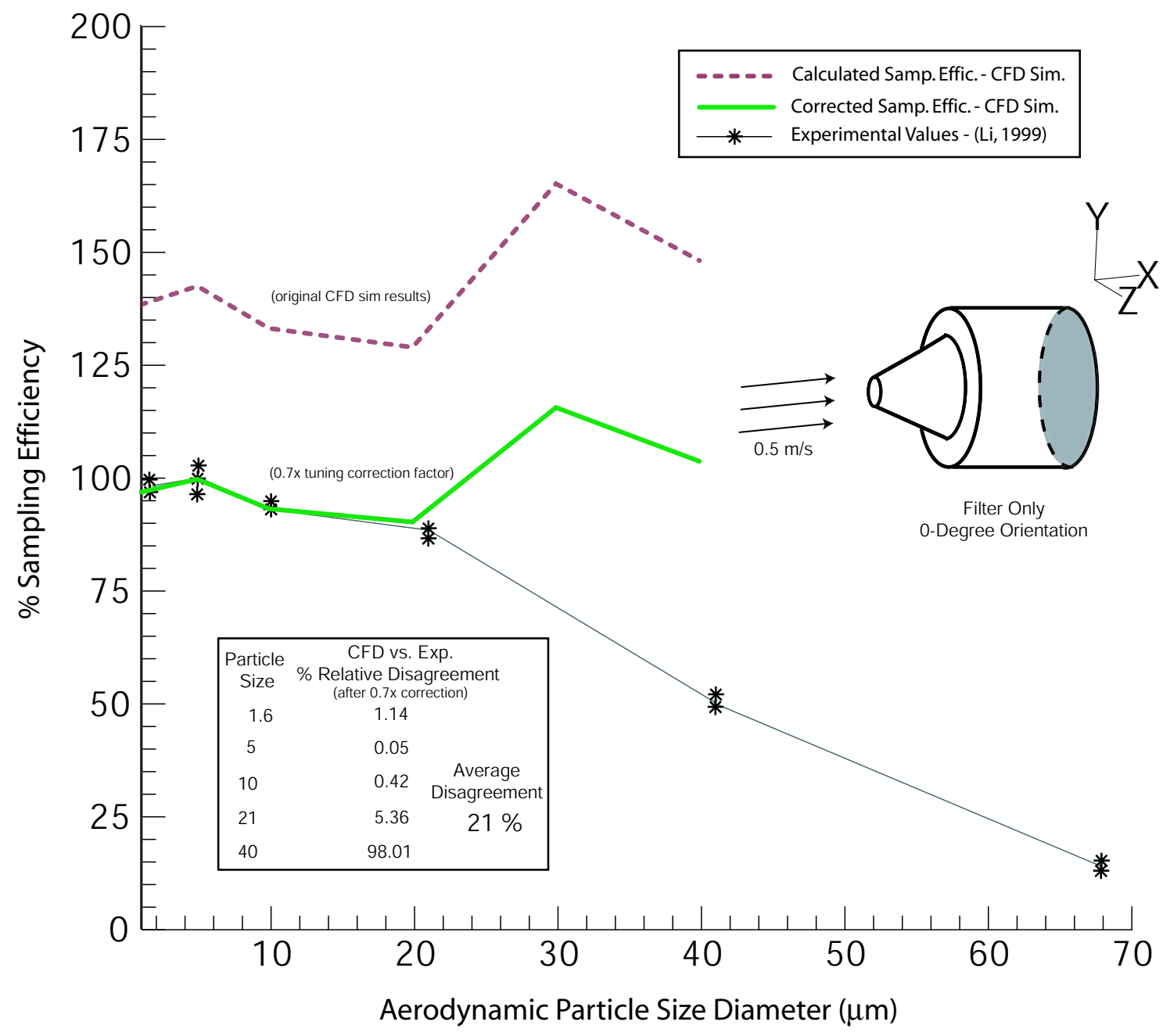

Figure 4.16: GSP sampling efficiency values from CFD simulation and experiments by Li (1999) for "filter-only" sampling at an orientation of 0 degrees and a freestream windspeed of $0.5 \mathrm{~m} / \mathrm{s}$. The tuning factor from calculated sampling efficiency to corrected sampling efficiency for the CFD simulation is 0.7 . 


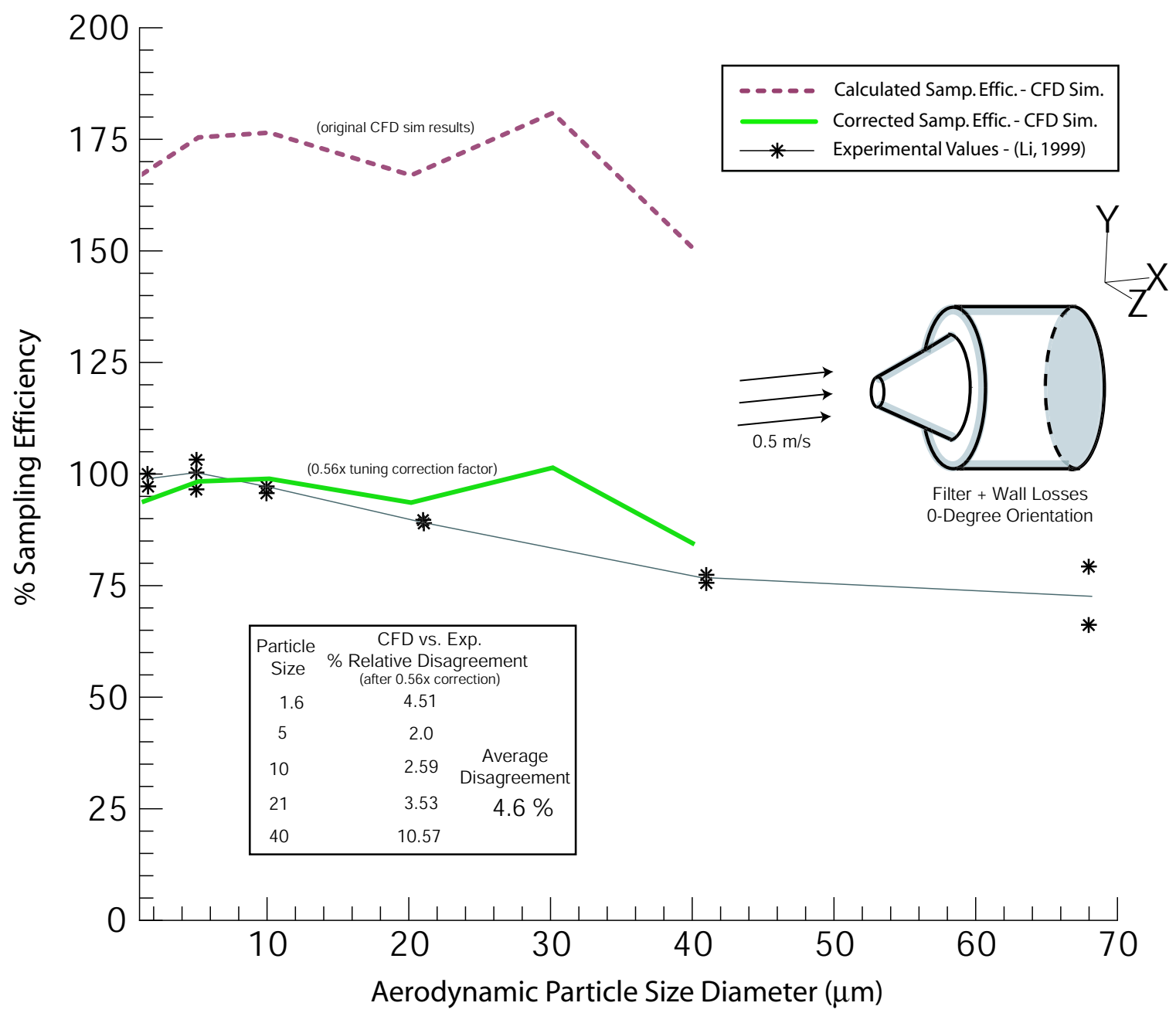

Figure 4.17: GSP sampling efficiency values from CFD simulation and experiments by Li (1999) for "total" sampling (filter + losses) at an orientation of 0 degrees and a freestream windspeed of $0.5 \mathrm{~m} / \mathrm{s}$. The tuning factor from calculated sampling efficiency to corrected sampling efficiency for the CFD simulation is 0.56 . 


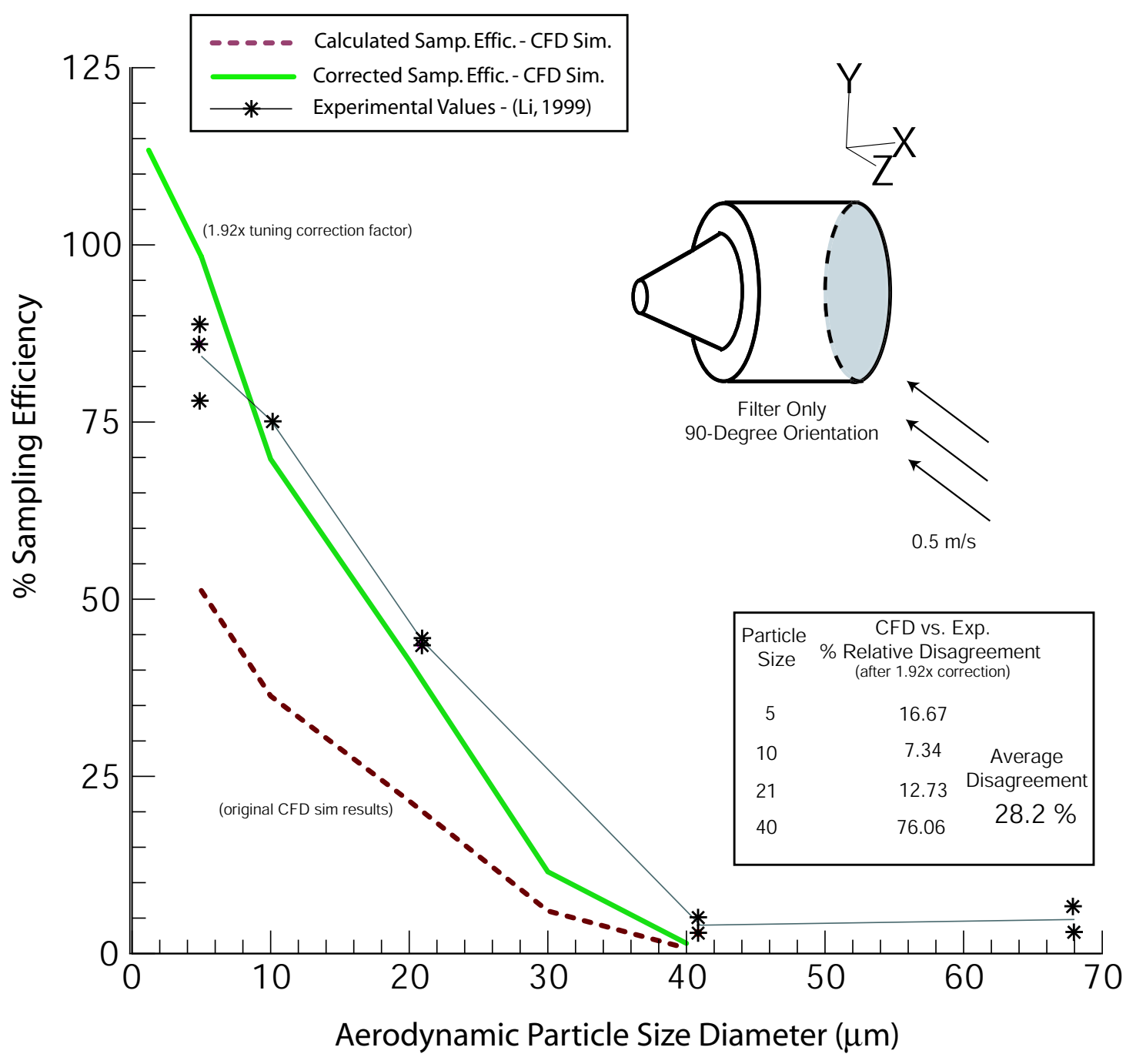

Figure 4.18: GSP sampling efficiency values from CFD simulation and experiments by Li (1999) for filter-only sampling at an orientation of 90 degrees and a freestream windspeed of $0.5 \mathrm{~m} / \mathrm{s}$. The tuning factor from calculated sampling efficiency to corrected sampling efficiency for the CFD simulation is 1.92 . 


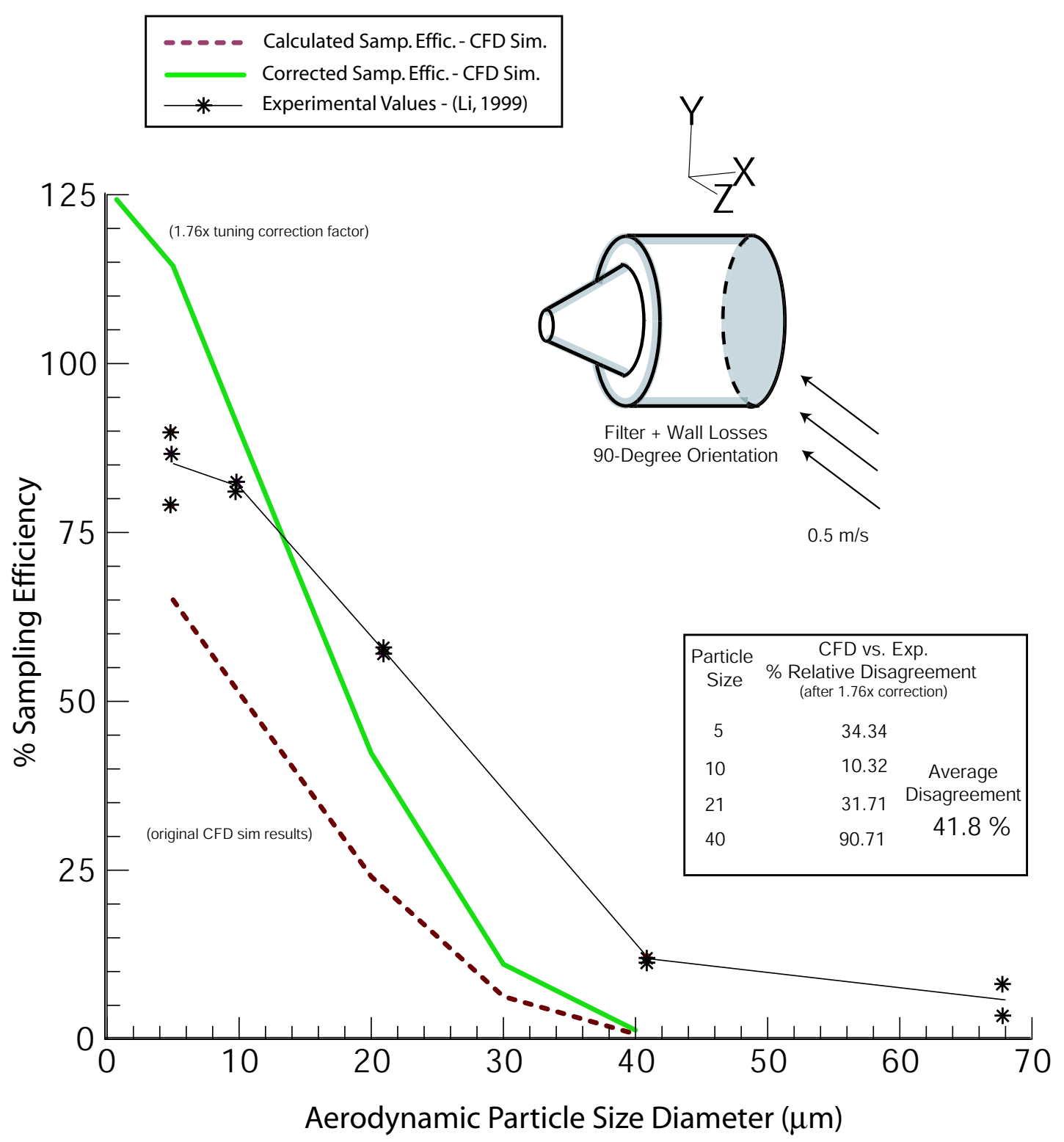

Figure 4.19: GSP sampling efficiency values from CFD simulation and experiments by Li (1999) for "total" sampling (filter + losses) at an orientation of 90 degrees and a freestream windspeed of $0.5 \mathrm{~m} / \mathrm{s}$. The tuning factor from calculated sampling efficiency to corrected sampling efficiency for the CFD simulation is 1.76 . 


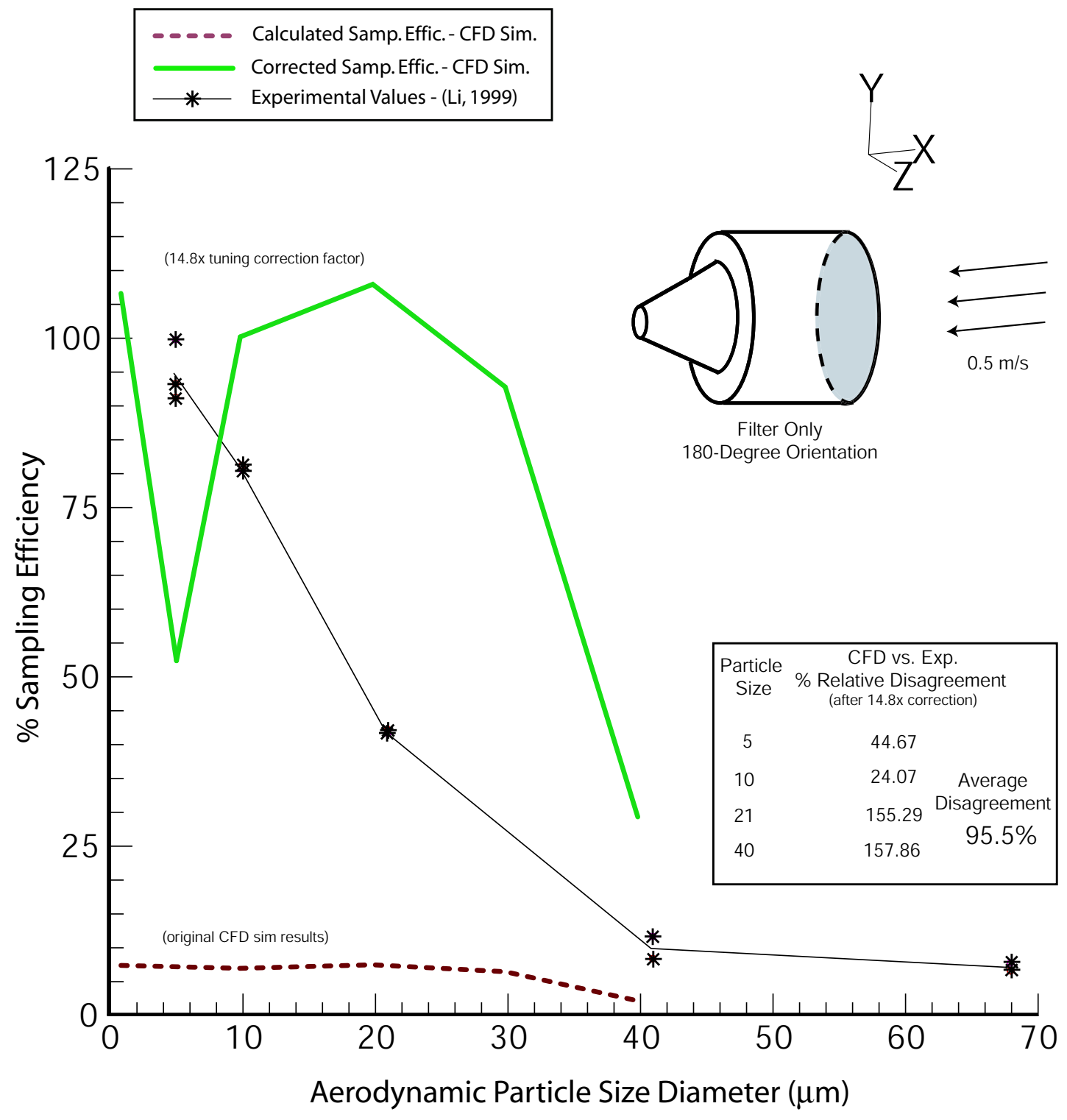

Figure 4.20: GSP sampling efficiency values from CFD simulation and experiments by Li (1999) for filter-only sampling at an orientation of 180 degrees and a freestream windspeed of $0.5 \mathrm{~m} / \mathrm{s}$. The tuning factor from calculated sampling efficiency to corrected sampling efficiency for the CFD simulation is 14.8 . 


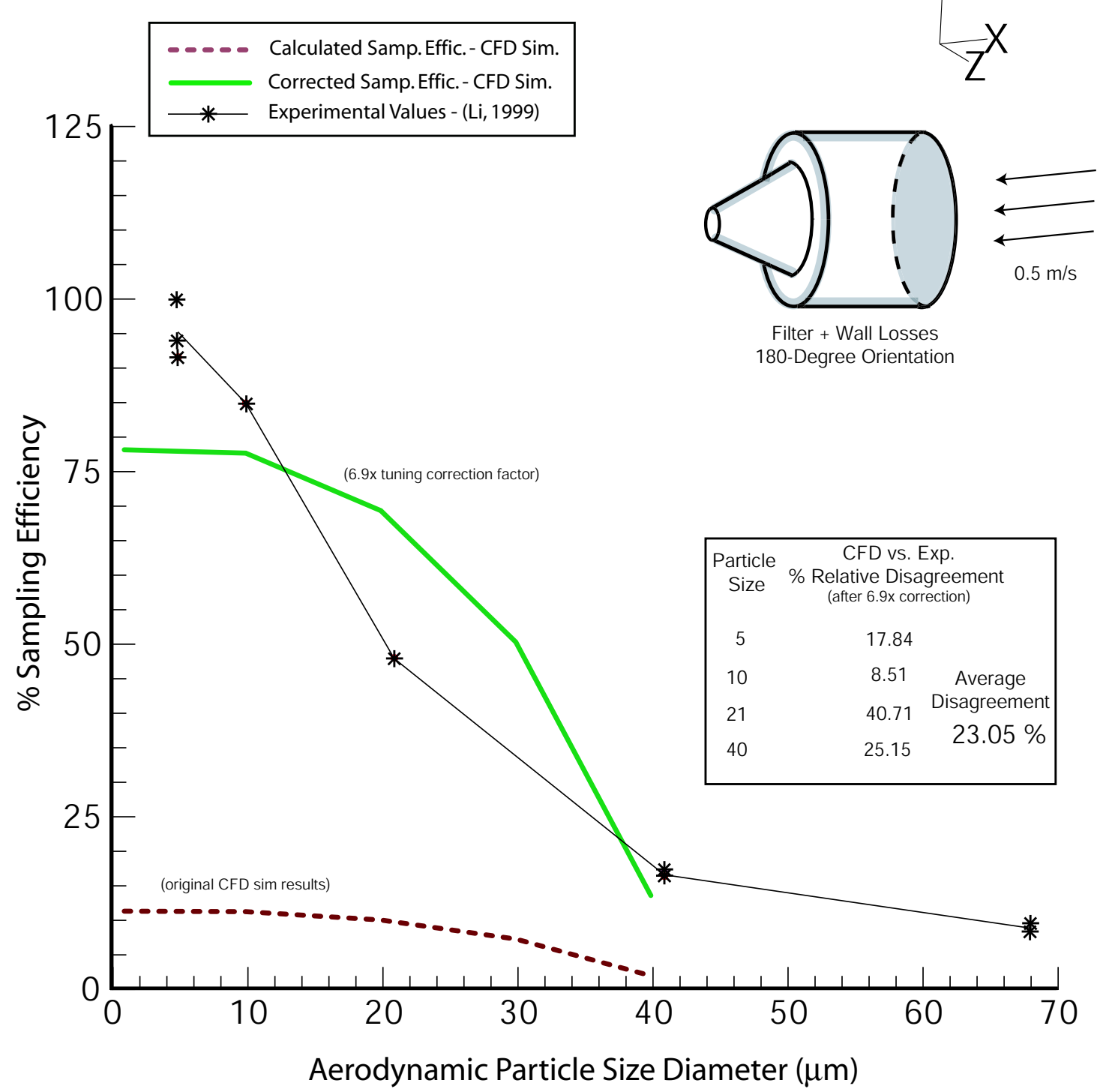

Figure 4.21: GSP sampling efficiency values from CFD simulation and experiments by Li (1999) for "total" sampling (filter + losses) at an orientation of 180 degrees and a freestream windspeed of $0.5 \mathrm{~m} / \mathrm{s}$. The tuning factor from calculated sampling efficiency to corrected sampling efficiency for the CFD simulation is 6.9. 


\section{Direction-Specific Results: 1 m/s Freestream Windspeed}

Simulations of the airflow and particle behavior near and into the GSP sampler were repeated for the $1 \mathrm{~m} / \mathrm{s}$ freestream velocity for $0-$, 90-, and 180-degree orientations. Figure 4.22 shows the results of corrected CFD simulation of the GSP sampler at a 0-degree orientation to $1 \mathrm{~m} / \mathrm{s}$ freestream flow for filter-only sampling. As with the $0.5 \mathrm{~m} / \mathrm{s}$ freestream flow, agreement is good for particle sizes up to $21 \mu \mathrm{m}$, but is poor at the $40-\mu m$ particle size. When wall losses are considered (Figure 4.23) agreement is much better for all particle sizes compared, where the overall average is $4.05 \%$.

Agreement for 90 -degree orientations in $1 \mathrm{~m} / \mathrm{s}$ freestream flow appears to be qualitatively good, but is somewhat high in several cases, even though the trends are very close for both filter-only (Figure 4.24) and total sampling (Figure 4.25). Overall averages for these simulations are 20.2 and $39.5 \%$, respectively.

At the 180-degree orientation for filter-only (Figure 4.26) and total-sampling (Figure 4.27) results at $1 \mathrm{~m} / \mathrm{s}$ freestream flow are, in appearance, similar to those at the $0.5 \mathrm{~m} / \mathrm{s}$ flows. Agreement for both conditions is reasonable up to $10 \mu \mathrm{m}$, but is poor for larger particle sizes. Finally, for filter-only and total-sampling at the 180-degree orientation in 1 $\mathrm{m} / \mathrm{s}$ freestream flow, large tuning correction factors are necessary to bring the calculated sampling efficiencies into reasonable agreement with experimental results. As discussed in the description of $0.5 \mathrm{~m} / \mathrm{s}$ freestream conditions, this is likely due to the low number of particles collected in this physical scenario. 


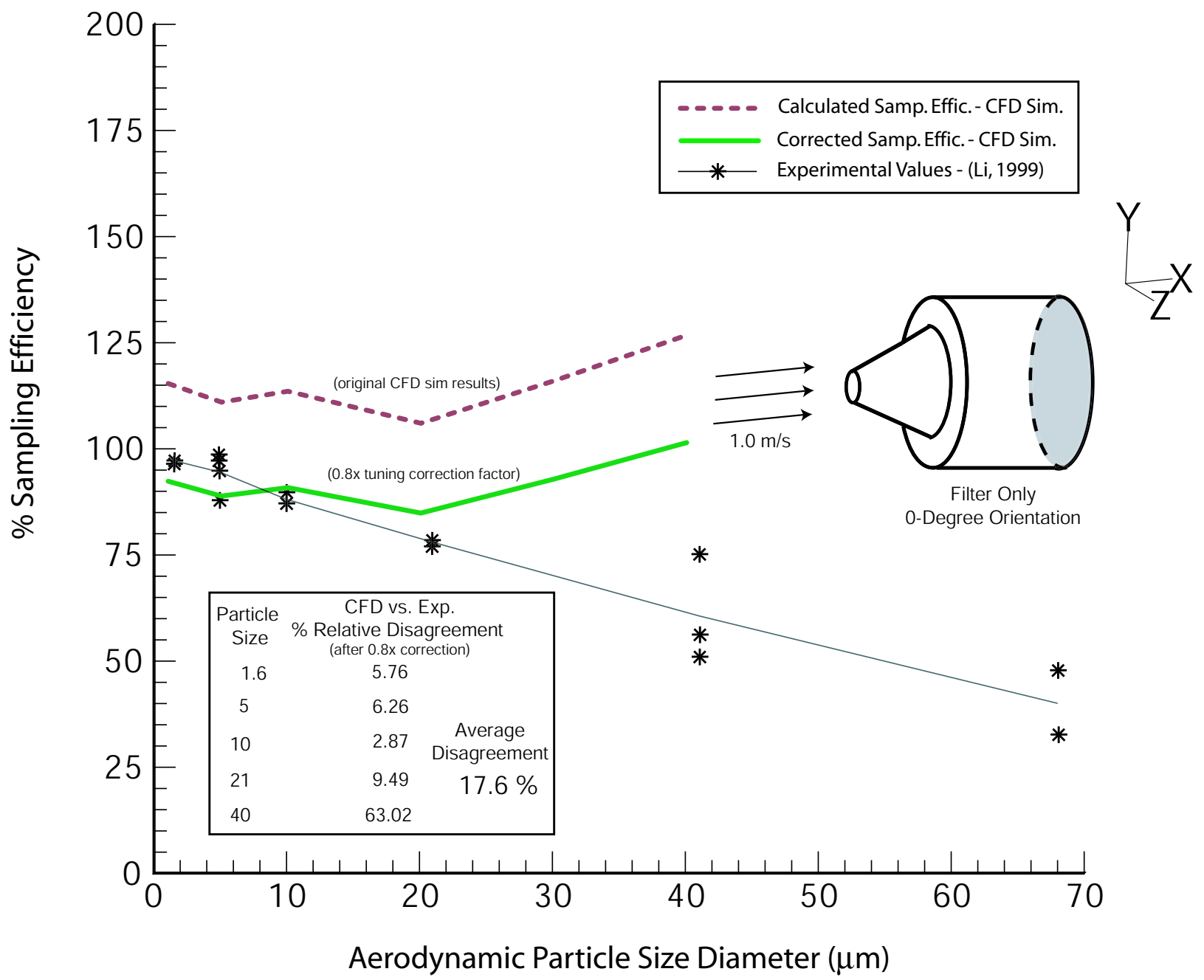

Figure 4.22: GSP sampling efficiency values from CFD simulation and experiments by Li (1999) for filter-only sampling at an orientation of 0 degrees and a freestream windspeed of $1 \mathrm{~m} / \mathrm{s}$. The tuning factor from calculated sampling efficiency to corrected sampling efficiency for the CFD simulation is 0.8 . 


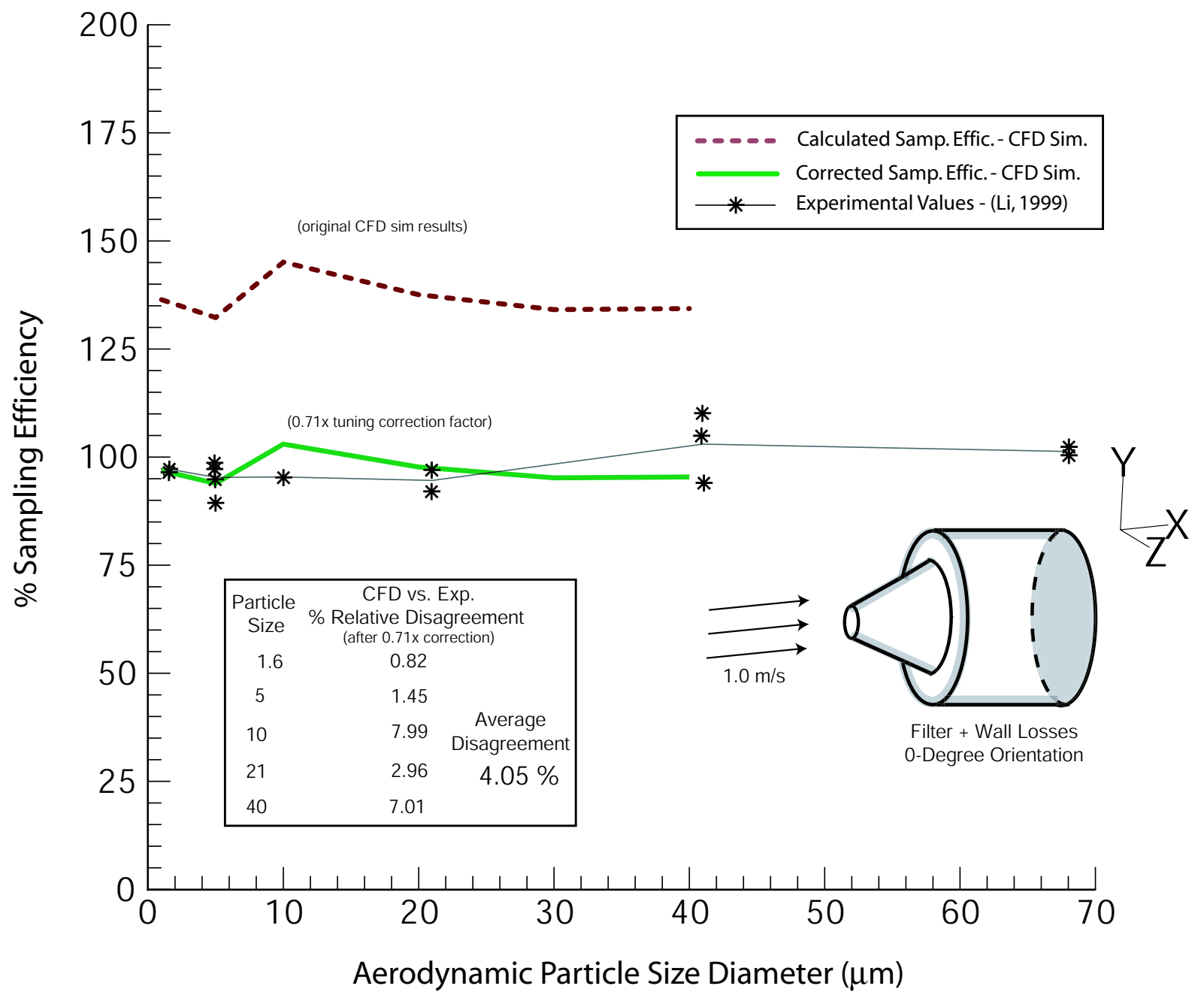

Figure 4.23: GSP sampling efficiency values from CFD simulation and experiments by Li (1999) for "total" sampling (filter + losses) at an orientation of 0 degrees and a freestream windspeed of $1 \mathrm{~m} / \mathrm{s}$. The tuning factor from calculated sampling efficiency to corrected sampling efficiency for the CFD simulation is 0.71 . 


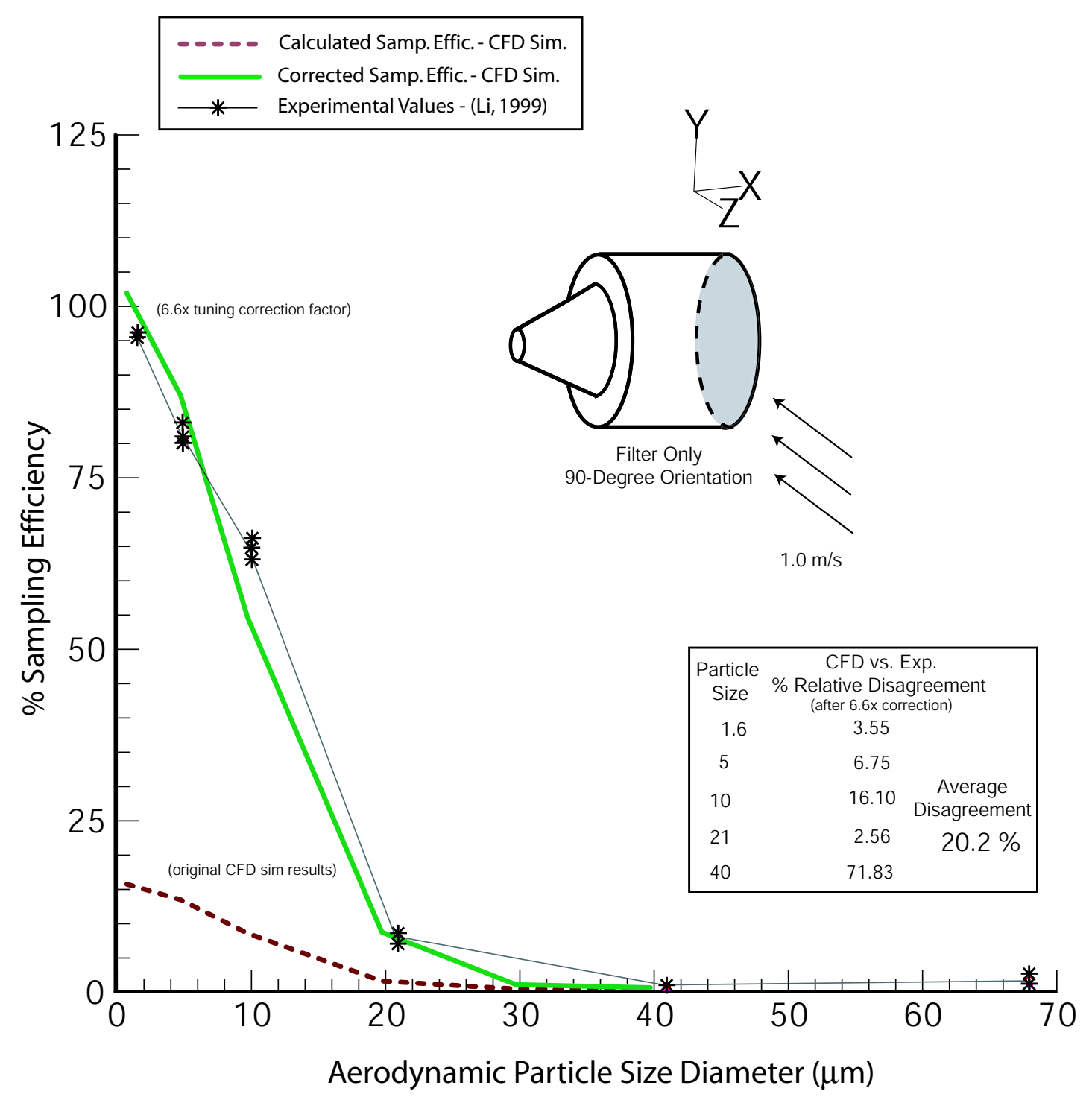

Figure 4.24: GSP sampling efficiency values from CFD simulation and experiments by Li (1999) for filter-only sampling at an orientation of 90 degrees and a freestream windspeed of $1.0 \mathrm{~m} / \mathrm{s}$. The tuning factor from calculated sampling efficiency to corrected sampling efficiency for the CFD simulation is 6.6. 


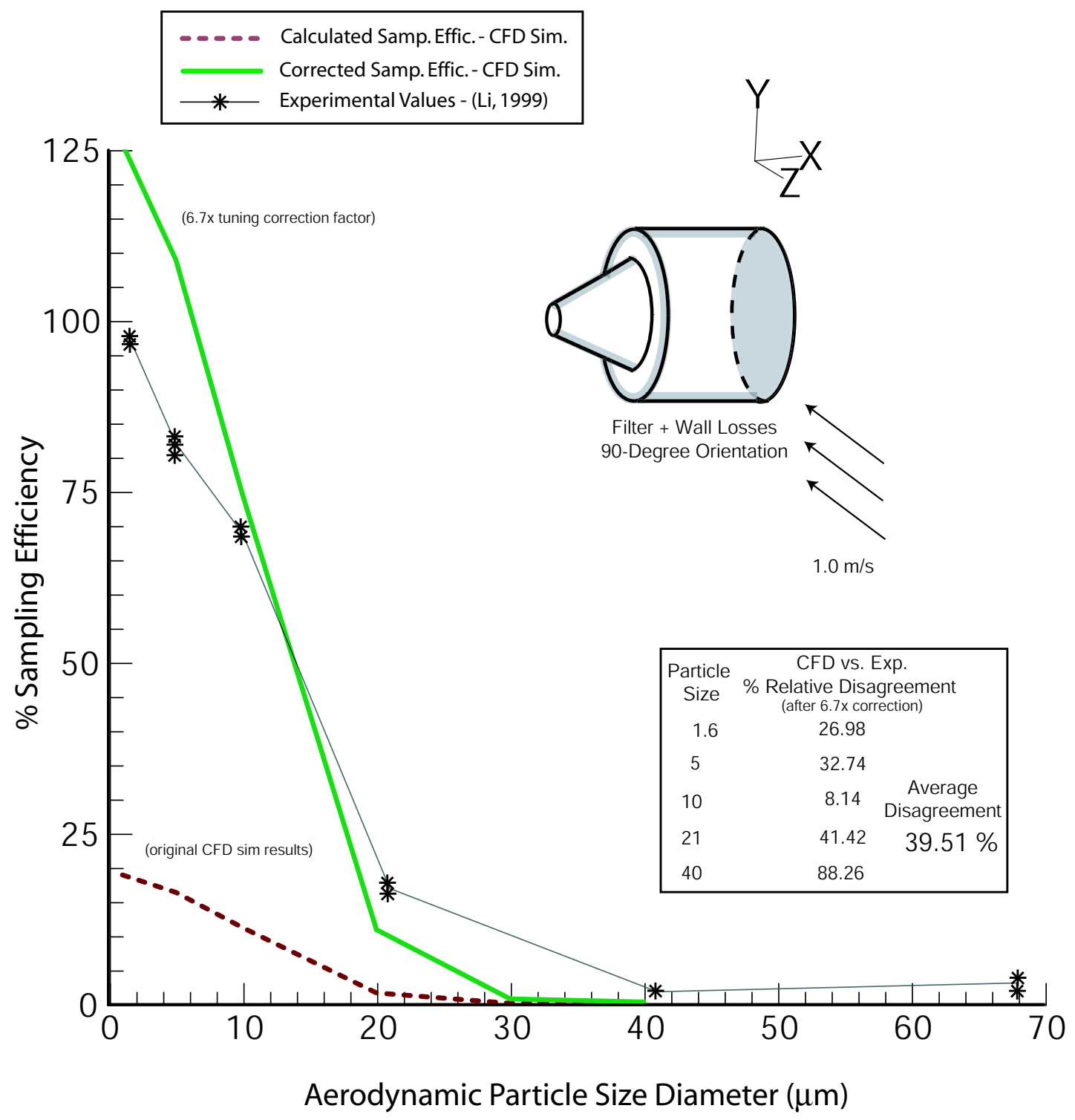

Figure 4.25: GSP sampling efficiency values from CFD simulation and experiments by Li (1999) for "total" sampling (filter + losses) at an orientation of 90 degrees and a freestream windspeed of $1.0 \mathrm{~m} / \mathrm{s}$. The tuning factor from calculated sampling efficiency to corrected sampling efficiency for the CFD simulation is 6.7. 


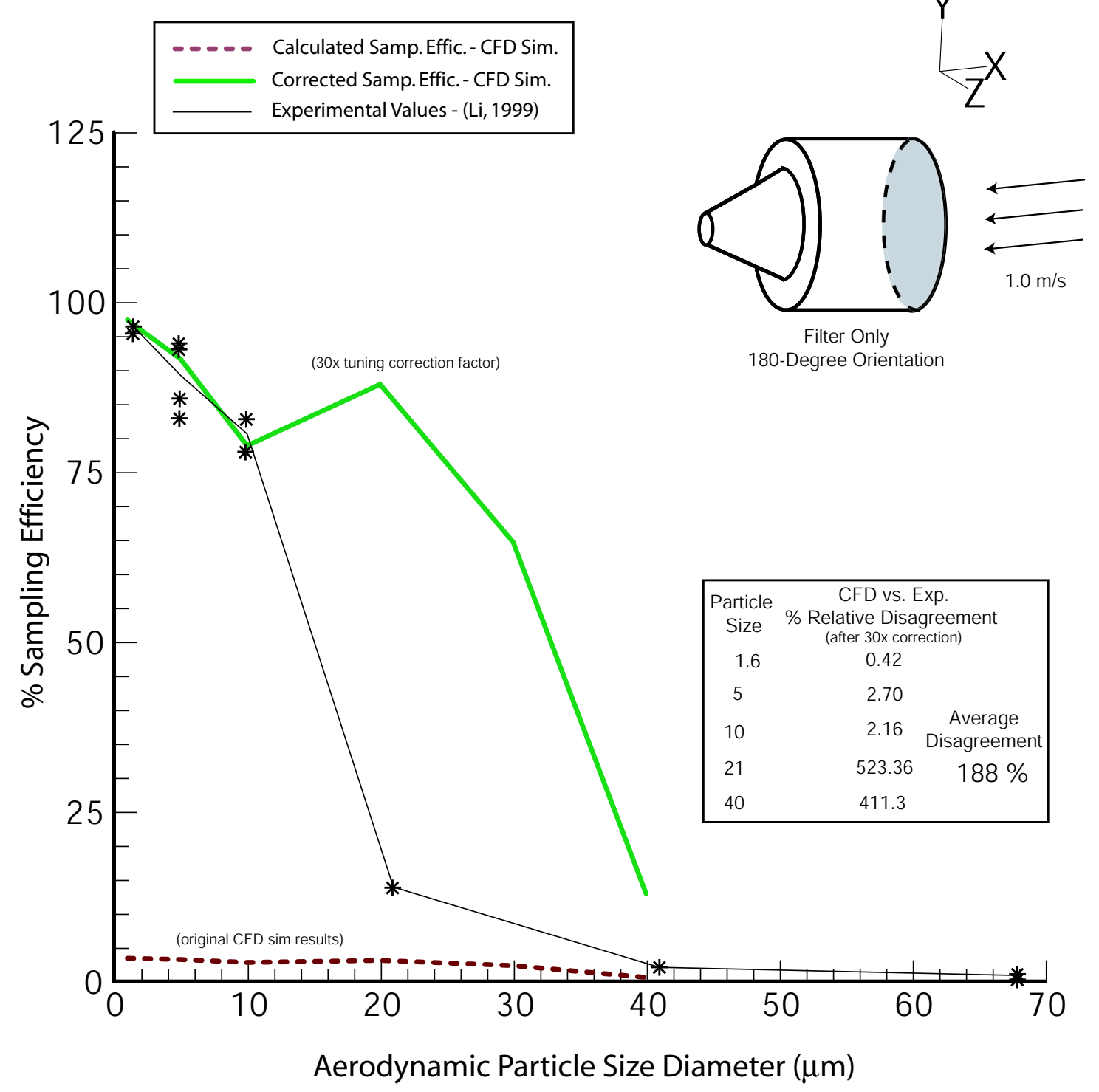

Figure 4.26: GSP sampling efficiency values from CFD simulation and experiments by Li (1999) for filter-only sampling at an orientation of 180 degrees and a freestream windspeed of $1.0 \mathrm{~m} / \mathrm{s}$. The tuning factor from calculated sampling efficiency to corrected sampling efficiency for the CFD simulation is 30 . 


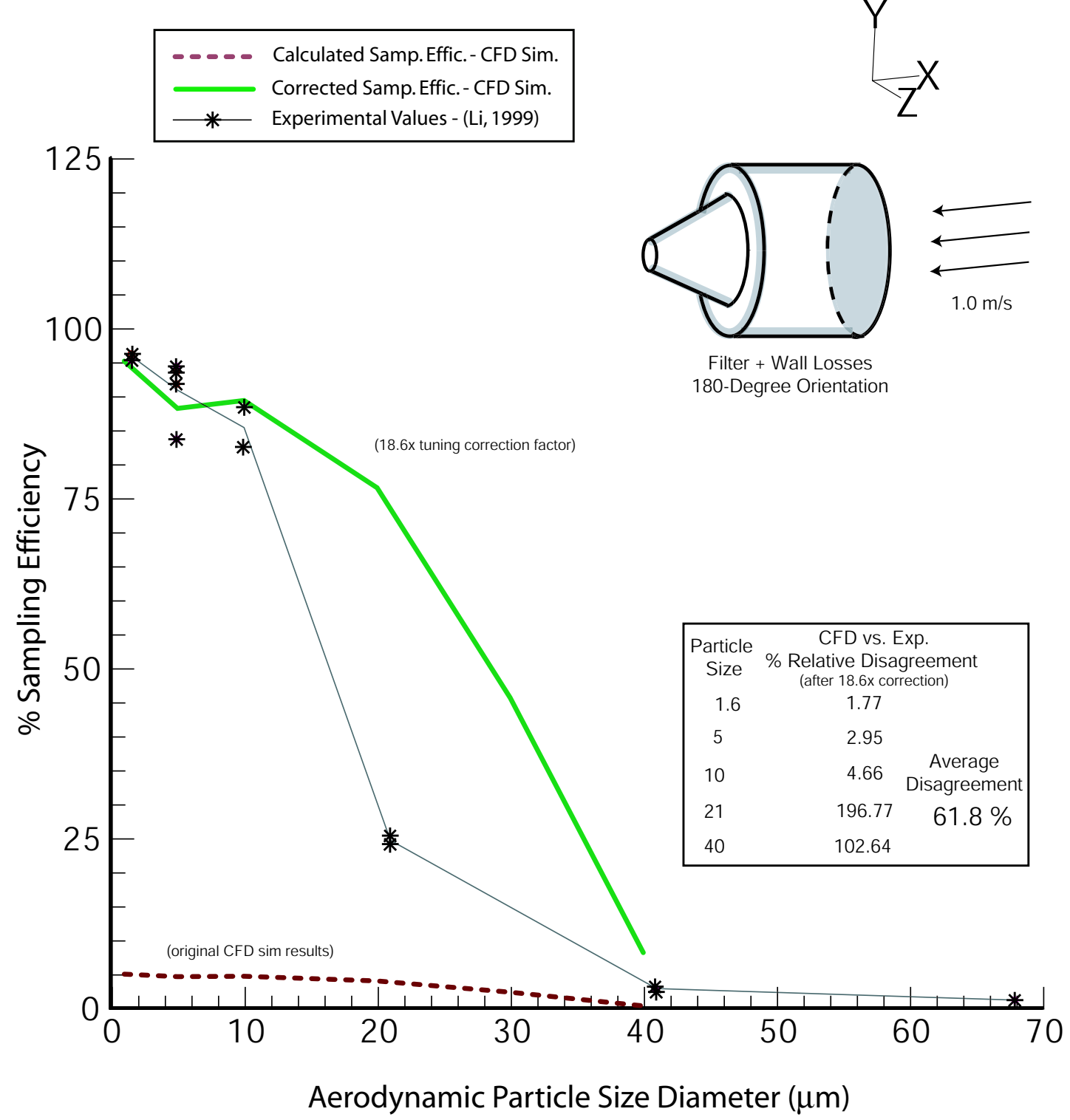

Figure 4.27: GSP sampling efficiency values from CFD simulation and experiments by Li (1999) for "total" sampling (filter + losses) at an orientation of 180 degrees and a freestream windspeed of $1.0 \mathrm{~m} / \mathrm{s}$. The tuning factor from calculated sampling efficiency to corrected sampling efficiency for the CFD simulation is 18.6. 


\section{Tuning Factors for GSP Sampler}

Tuning correction factors were used to bring the sampling efficiency results from CFD simulation into reasonable agreement with the experimental results (Table 4.4). "Reasonable agreement" was determined by averaging the percent relative differences between CFD and experiment for particle sizes from 1 to $20 \mu \mathrm{m}$. A different tuning correction factor was applied for each scenario, such that each factor was used only once on all of the results in that particular physical scenario. This was done to ensure that tuning did not replace simulation as a modeling method.

Comparison of the tuning correction factors reveals that there are common tuning factors that may be applied to more than one physical scenario. Selections of common tuning factors were made if the difference between two or more of them was not greater than $+/-25 \%$. For cases where this condition was true, the average of the two (or more) values was used as a common tuning correction factor. Since results of sampling efficiency determinations for total sampling were little better than those for filter sampling, only for the latter were common tuning factors determined.

Table 4.5 shows the tuning factors averaged by windspeed. In the table it can be seen that average relative differences are small for the 0-degree orientation, but large for the 90 and 180-degree orientations. Thus, only for the 0-degree orientation can the same tuning factor be used for both 0.5 and $1.0 \mathrm{~m} / \mathrm{s}$ freestream flows. At the other orientations, separate tuning factors are necessary for each orientation at the two windspeeds, i.e. the values from Table 4.4. 
Table 4.4: Correction tuning factors for calculated sampling efficiencies of the GSP sampler based on experimental values from Li (1999) together with the resulting average percent relative disagreement for that condition.

\begin{tabular}{|c|c|c|c|c|c|}
\hline \multirow{2}{*}{ Orientation } & Windspeed & Factor & Disagreement & Factor & Disagreement \\
& & Total S.E. & & Filter S.E. & \\
0 & 0.5 & 0.56 & 5.0 & 0.7 & 21 \\
0 & 1.0 & 0.71 & 4.1 & 0.8 & 17.6 \\
90 & 0.5 & 1.76 & 41.8 & 1.92 & 28.2 \\
90 & 1.0 & 6.7 & 39.5 & 6.6 & 20.02 \\
180 & 0.5 & 6.9 & 23.1 & 14.8 & 95.5 \\
180 & 1.0 & 18.6 & 61.8 & 30.0 & 188 \\
\hline
\end{tabular}


Table 4.5: Average correction tuning factors for the GSP sampler at 0-, 90-, and 180-degree orientations to freestream flows of 0.5 and $1.0 \mathrm{~m} / \mathrm{s}$ for filter-only sampling with deviation from original values of relative percent disagreement in parentheses.

\begin{tabular}{|c|c|c|c|}
\hline & & Tuning Factor & Avg. \% Rel. \\
Orientation & Windspeed & Filter S.E. & Disagreement \\
0 & 0.5 & 0.75 & $29.0(+8.0)$ \\
0 & 1.0 & 0.75 & $16.3(-1.3)$ \\
90 & 0.5 & 4.26 & $108.7(+80.5)$ \\
90 & 1.0 & 4.26 & $43.3(+23.1)$ \\
180 & 0.5 & 22.4 & $170.5(+75.0)$ \\
180 & 1.0 & 22.4 & $144.41(-43.6)$ \\
& & & \\
\hline
\end{tabular}




\section{GSP Mounted on Box-Shaped Bluff Body}

\section{Direction-Averaged Results}

By convention, aerosol samplers have been compared to the Inhalability curve in order to evaluate their performance. Traditionally this has involved measuring the sampling efficiency of aerosol samplers on rotating full-size mannequins in large-scale wind tunnels. However, the work of Witschger et al. (1998) and Aizenberg et al. (2000) has demonstrated that a full-size rotating torso is not needed. Instead, the average of directional orientations of the aerosol sampler mounted on a box-shaped bluff body in multiple orientations of freestream flow in a small-scale wind tunnel can be taken and used as the value with which comparison can be made against the Inhalability curve. The averaging method used by Witschger and Aizenberg is

$$
E_{T O T A L}=\left(a E_{0}+b E_{90}+c E_{180}\right)
$$

where $E_{T O T A L}$ is the total sampling efficiency, $a=0.25, b=0.5$, and $c=0.25$. The equation is essentially an average of sampling results if a sampler were placed on each side of the box-shaped bluff body. Equation 4.1 is also referred to in this text as "Witschger Averaging." In addition a simple average using equation 4.2, referred to as "3X Averaging" can also be taken for comparison purposes.

$$
E_{T O T A L}=\frac{1}{3}\left(E_{0}+E_{90}+E_{180}\right)
$$

The computational domain for the GSP sampler mounted on a box-shaped bluff body is much larger than for the GSP sampler alone. In addition, the reference probe must be 
placed far enough away so there is no flow disturbance on the probe due to the bluff body. These two factors result in computational grids approaching the maximum allowed by the 32-bit hardware, which is approximately 5 million cells. The box-shaped bluff body is based on the "simplified manikin" from Aizenberg et al. (2000), which has dimensions of $0.33 \mathrm{~m}$ wide, $0.21 \mathrm{~m}$ high, and $0.21 \mathrm{~m}$ thick.

If not for the influence of gravity, the reference probe could be placed above the simplified manikin, where the axis is shorter, thereby reducing the number of grid cells required. However, the effect of gravity on the particles would require a very "tall" (or long) release face so as to normalize the effect on sampling flux. This is unrealistic, so the reference probe must be placed horizontally to the right or left of the box-shaped bluff body and then exposed to the same particle concentration as that delivered to the GSP sampler. In addition, it is currently (2004) best to avoid use of symmetry planes in FLUENT where the discrete phase model will be used because particles may be "lost" through such a face thereby adversely affecting the number of particles in the domain. This has been confirmed through direct observation, but not investigated quantitatively.

CFD simulations were conducted as previously described with slight modifications to the k-e turbulence model usage for 0.5 and $2.0 \mathrm{~m} / \mathrm{s}$ freestream velocities. Particle trajectories were done as described previously for particle sizes of 1, 6, 10, 16, 20, and 50 micrometers.

Figure 4.28 shows the results of $3 \mathrm{X}$ direction-averaged sampling efficiency (equation 4.2) predicted by CFD for the GSP sampler in a windspeed of $0.5 \mathrm{~m} / \mathrm{s}$. The CFD results were tuned to the Inhalability convention curve by a factor of 1.5 (see Table 4.6 for complete 
tuning constants). Also shown in this plot are experimental data (average value, $+/$ - one standard deviation) from Kenny et al. (1997) at $0.5 \mathrm{~m} / \mathrm{s}$ for a similar range of particle sizes. Comparisons of percent relative disagreements were made these are shown in the table in Figure 4.28. The disagreement is rather large in most cases, with particularly large discrepancies at 6 and $16 \mu \mathrm{m}$. The cause for these discrepancies in the apparent trend of the CFD results is not clear. However, several possible reasons are human error, too few particles in computational domain to yield accurate results, and/or software error (mentioned above).

Figure 4.29 shows the same data also corrected by a factor of 1.5 , but for Witscgher averaging (equation 4.1). It can be seen from the plot that the results are shifted down and the extremes of the discrepancies are reduced. This is due to the extra weighting of the 90-degree orientation value, which has a relatively low sampling efficiency thus have the effect of additional reduction on direction-averaged sampling efficiency. Witschger averaging also results in closer agreement with Kenny's experimental results.

Additional simulations were made at the 1.25 and $2.0 \mathrm{~m} / \mathrm{s}$ freestream velocities and these are shown in Figure 4.30 with measurements from Kenny et al. (1997) made at 1.25 and $4 \mathrm{~m} / \mathrm{s}$. A qualitative conclusion can be made that the corrected CFD simulations fall into the proper location on the plot based on the plotted locations of Kenny's results. Direct comparisons of relative percent difference cannot be made.

All of the CFD simulation results were tuned to the Inhalability convention curve by relatively consistent tuning factors ranging from 1.5 to 2.5 (see Table 4.6). The largest dif- 
ference in tuning factors was for Witschger averaging for the $0.5 \mathrm{~m} / \mathrm{s}$ freestream windspeed to the $1.25 \mathrm{~m} / \mathrm{s}$ windspeed. Thus, in order to determine if a single tuning correction factor could be applied to all of the physical scenarios, the Witschger averaging CFD results were tuned by a factor of 2.5 (see Table 4.7). This resulted in an average percent relative disagreement identical to that when 1.5 was used as the tuning factor, although individual disagreements were generally larger. It is reasonable to use the single value of 2.5 for tuning results of Witschger averaging for CFD simulations of all three freestream windspeeds. 


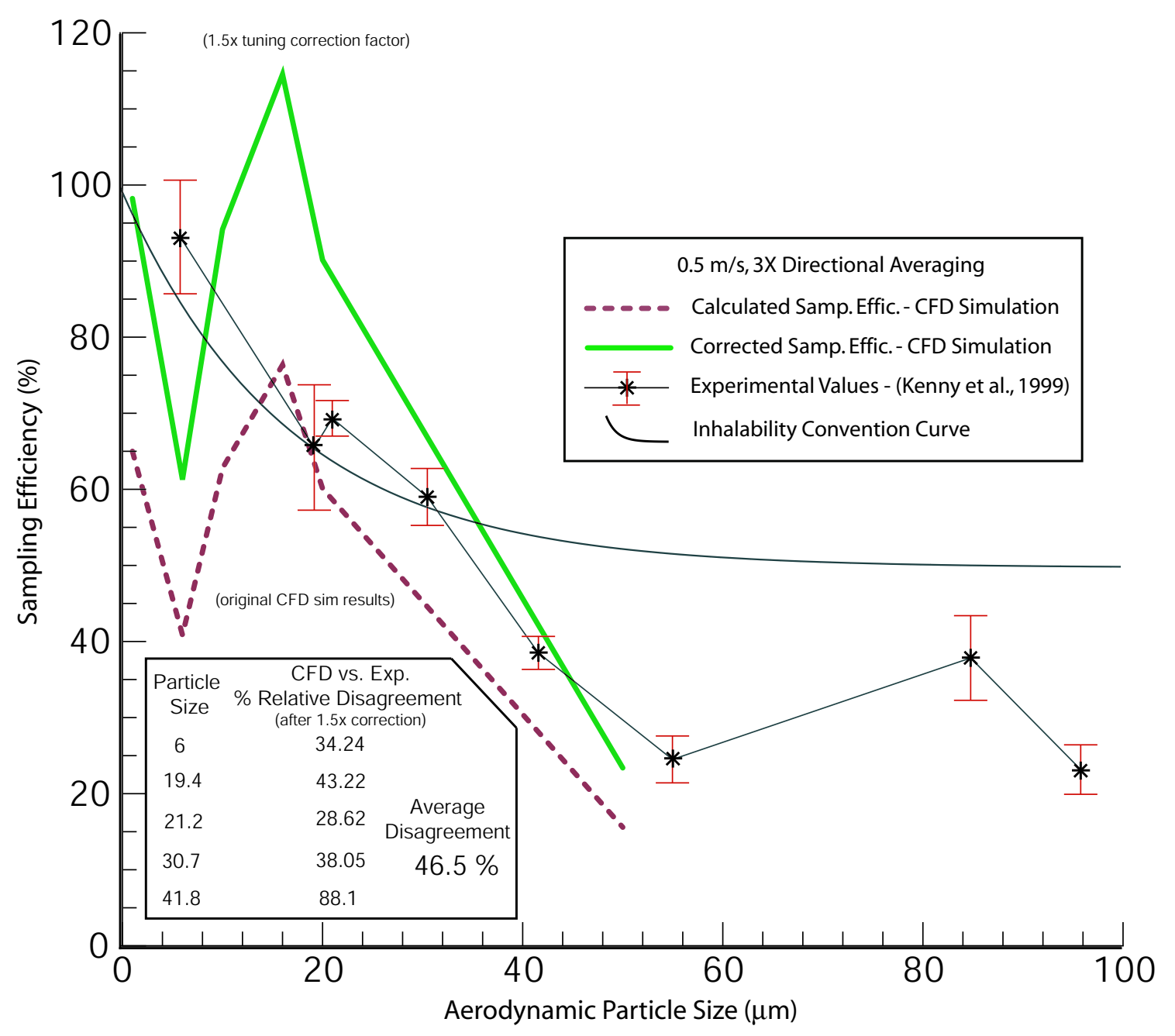

Figure 4.28: $3 \mathrm{X}$ direction-averaged sampling efficiency values for GSP sampler mounted on box-shaped bluff body in freestream windspeed of $0.5 \mathrm{~m} / \mathrm{s}$. 


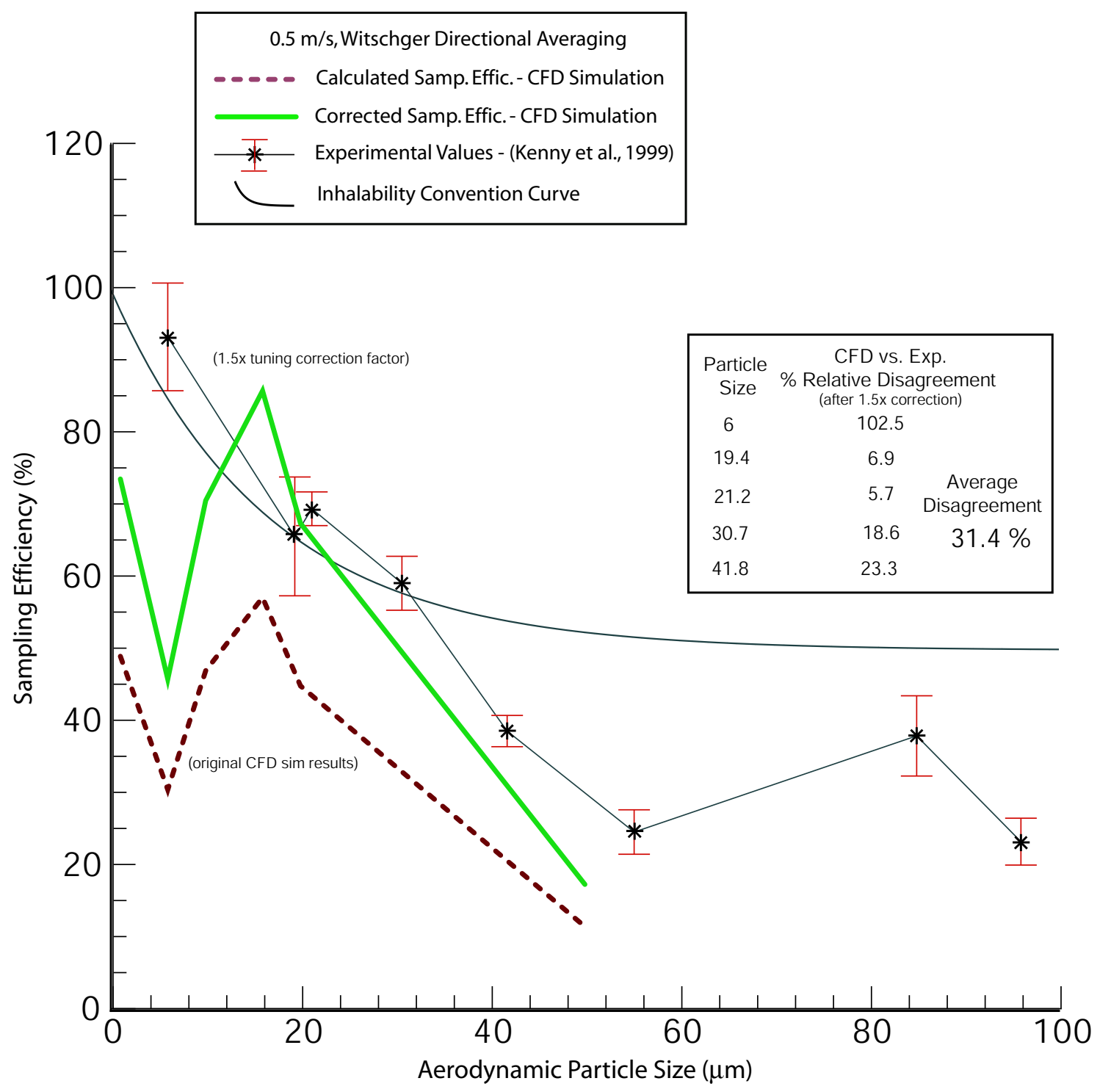

Figure 4.29: Witschger direction-averaged sampling efficiency values for GSP sampler mounted on box-shaped bluff body in freestream windspeed of $0.5 \mathrm{~m} / \mathrm{s}$ with experimental values from Kenny et al. (1997). 


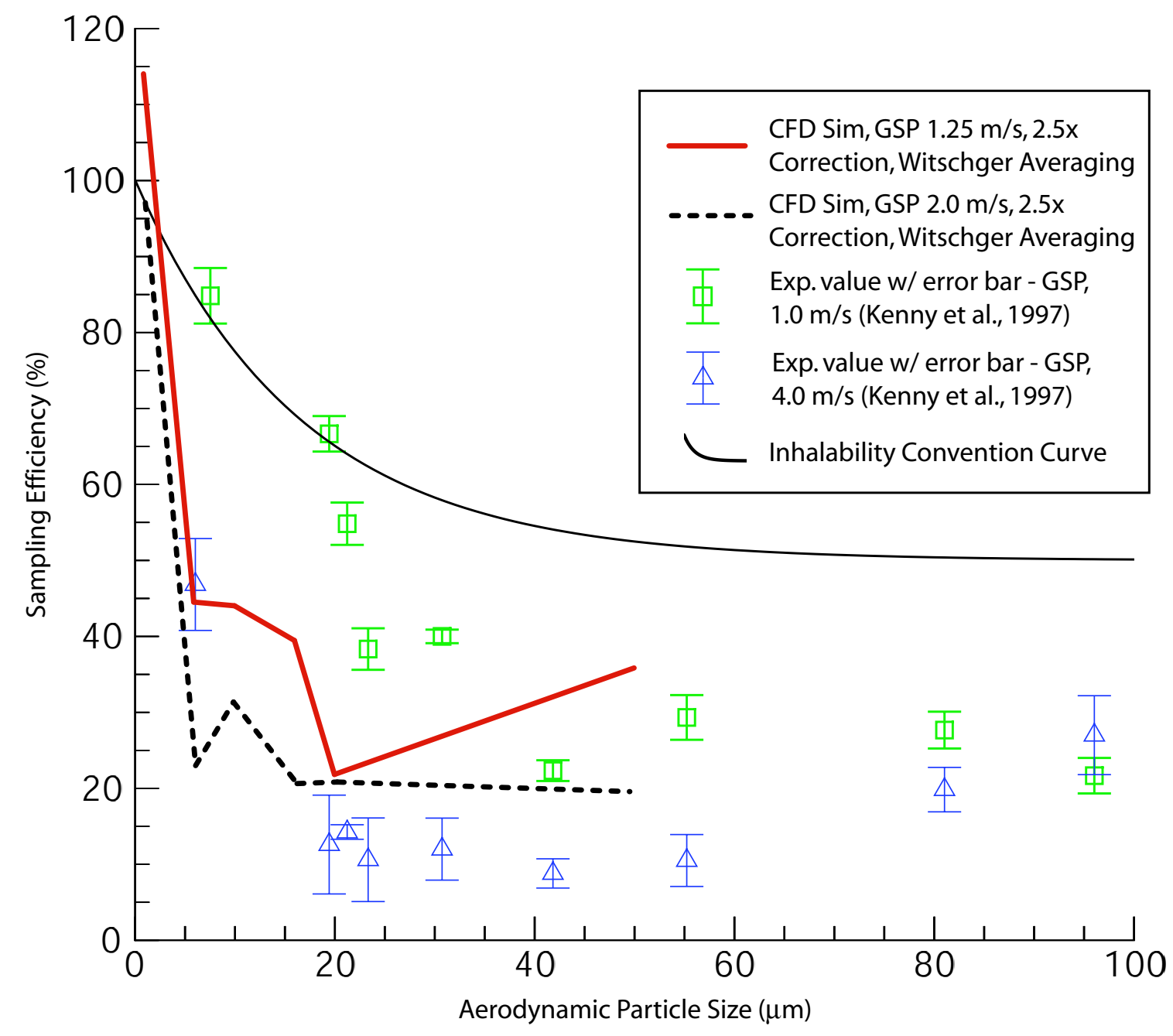

Figure 4.30: Witschger direction-averaged sampling efficiency values for GSP sampler mounted on box-shaped bluff body in freestream windspeeds of 1.25 and $2.0 \mathrm{~m} / \mathrm{s}$ with experimental values from Kenny et al. (1997). 
Table 4.6: Correction tuning constants, based on Inhalability convention, and the operation function on their respective data sets from simulations of the GSP sampler mounted on the box-shaped bluff body with experimental values from Kenny et al. (1997).

\begin{tabular}{|c|c|c|l|}
\hline $\begin{array}{c}\text { Windspeed } \\
(\mathrm{m} / \mathrm{s})\end{array}$ & $\begin{array}{c}\text { Tuning Factor } \\
\text { 3X Avg. } \\
\text { (Equation 4.2) }\end{array}$ & $\begin{array}{c}\text { Tuning Factor } \\
\text { Witschger Avg. } \\
\text { (Equation 4.1) }\end{array}$ & Function \\
\hline 0.5 & 1.5 & 1.5 & Multiplied \\
1.25 & 1.5 & 2.5 & Multiplied \\
2.0 & 1.9 & 2.5 & Multiplied \\
\hline
\end{tabular}


Table 4.7: Percent relative disagreement of Witschger averaged simulation results for the GSP sampler at $0.5 \mathrm{~m} / \mathrm{s}$.

\begin{tabular}{|c|c|c|}
\hline Particle & \% Rel. Dis. & \% Rel. Dis. \\
Size & Factor = 1.5 & Factor = 2.5 \\
$(\mu m)$ & & \\
\hline 6 & 102.5 & 21.5 \\
19.4 & 6.9 & 44.2 \\
21.2 & 5.7 & 36.6 \\
30.7 & 18.6 & 28.8 \\
41.8 & 23.3 & 26.0 \\
& Avg. $=31.4$ & Avg. $=31.4$ \\
\hline
\end{tabular}




\subsubsection{IOM Inhalable Personal Sampler}

Particle trajectories were calculated for the IOM sampler as they were for the GSP both free in the air and on the box-shaped bluff body. Sampling efficiencies were determined using the same sampler/probe flux method as previously described. For the IOM sampler free in the air, results were tuned with direction-specific experimental data from $\mathrm{Li}$ (1999). For the box-mounted IOM sampler, results were tuned with discrete values from the Inhalability convention.

\section{IOM Free in the Air}

\section{Direction-Specific Results: $0.5 \mathrm{~m} / \mathrm{s}$ Freestream Windspeed}

Results of tuned, direction-specific sampling efficiencies are plotted with experimental values in Figures 4.31 through 4.33 for orientations of 0-, 90-, and 180-degrees to $0.5 \mathrm{~m} / \mathrm{s}$ freestream airflow. Correction tuning constants were applied as described in the previous section for the GSP sampler and are shown in Table 4.9.

Sampling efficiencies for the IOM sampler are all 'total' values for several reasons. First, the designers of the sampler intended for all particles entering the sampler to be counted. Second, the Li data do not separate out the wall losses and filter collection values. Finally, creation of a computational geometry correctly representing the thin-walled inlet of the sampler is too expensive for the computational hardware available during this work. 
As seen in Figures, agreement with the Li data are generally reasonable up to $20 \mu \mathrm{m}$ for all three orientations. Agreement is good for the 0-degree orientation (Figure 4.31), less so for the 90-degree orientation (Figure 4.32), and reasonable at the 180-degree orientation (Figure 4.33). 


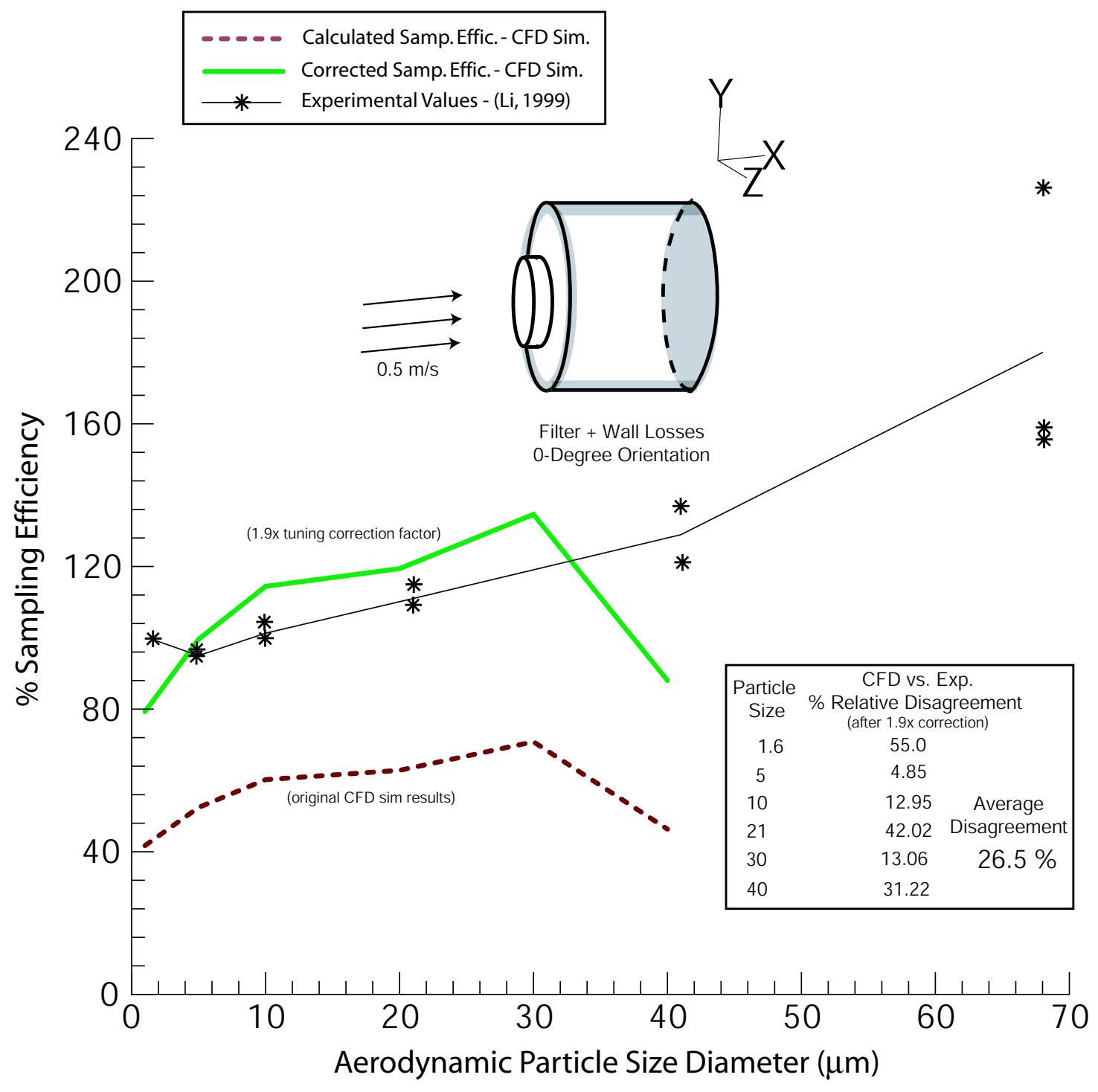

Figure 4.31: Sampling efficiencies of IOM sampler in comparison with experimental results from $\mathrm{Li}$ (1999) for the sampler free in the air and oriented 0 degrees to a $0.5 \mathrm{~m} / \mathrm{s}$ freestream airflow. 


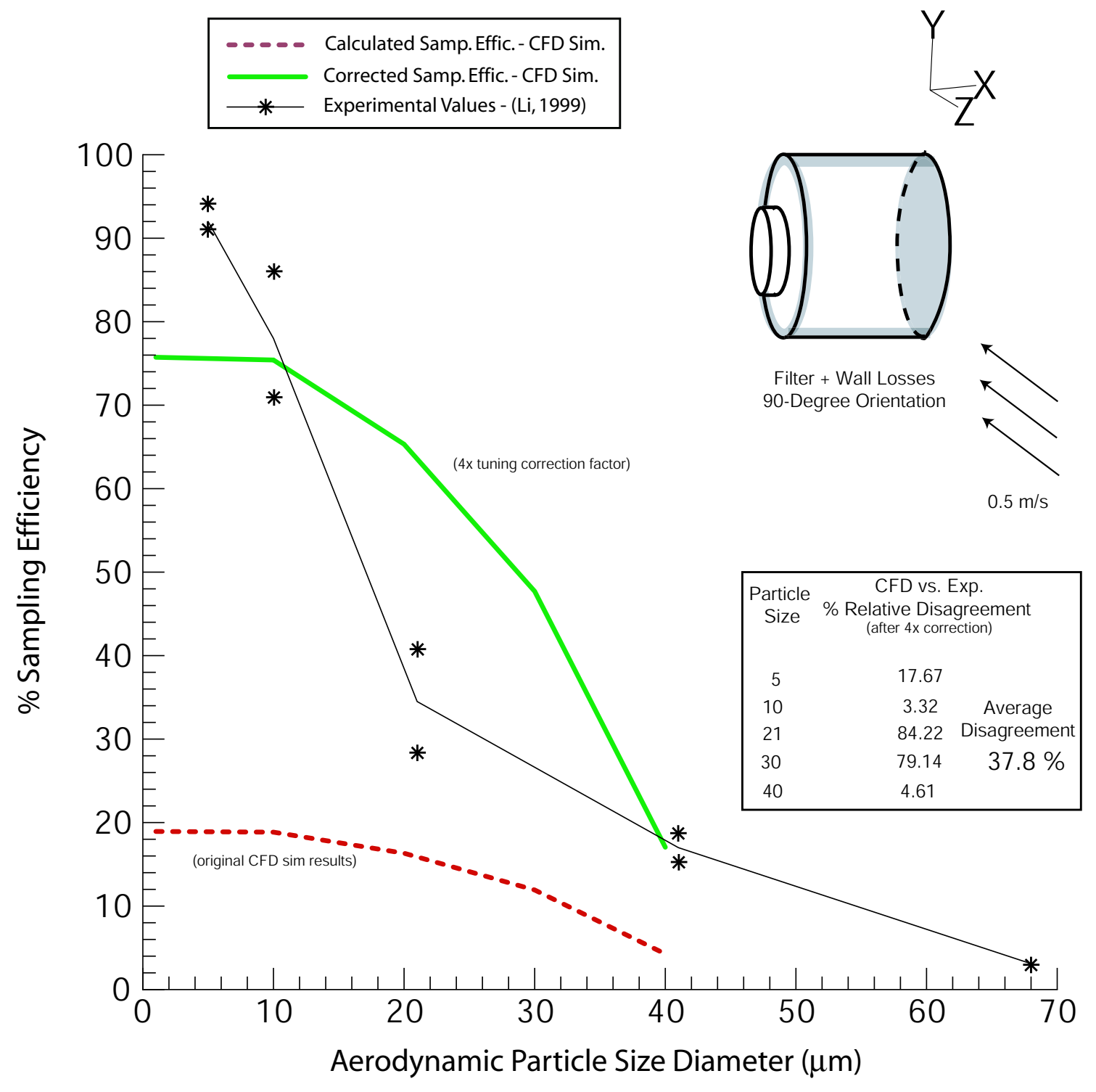

Figure 4.32: Sampling efficiencies of IOM sampler in comparison with experimental results from $\mathrm{Li}$ (1999) for the sampler free in the air and oriented 90 degrees to a $0.5 \mathrm{~m} / \mathrm{s}$ freestream airflow. 


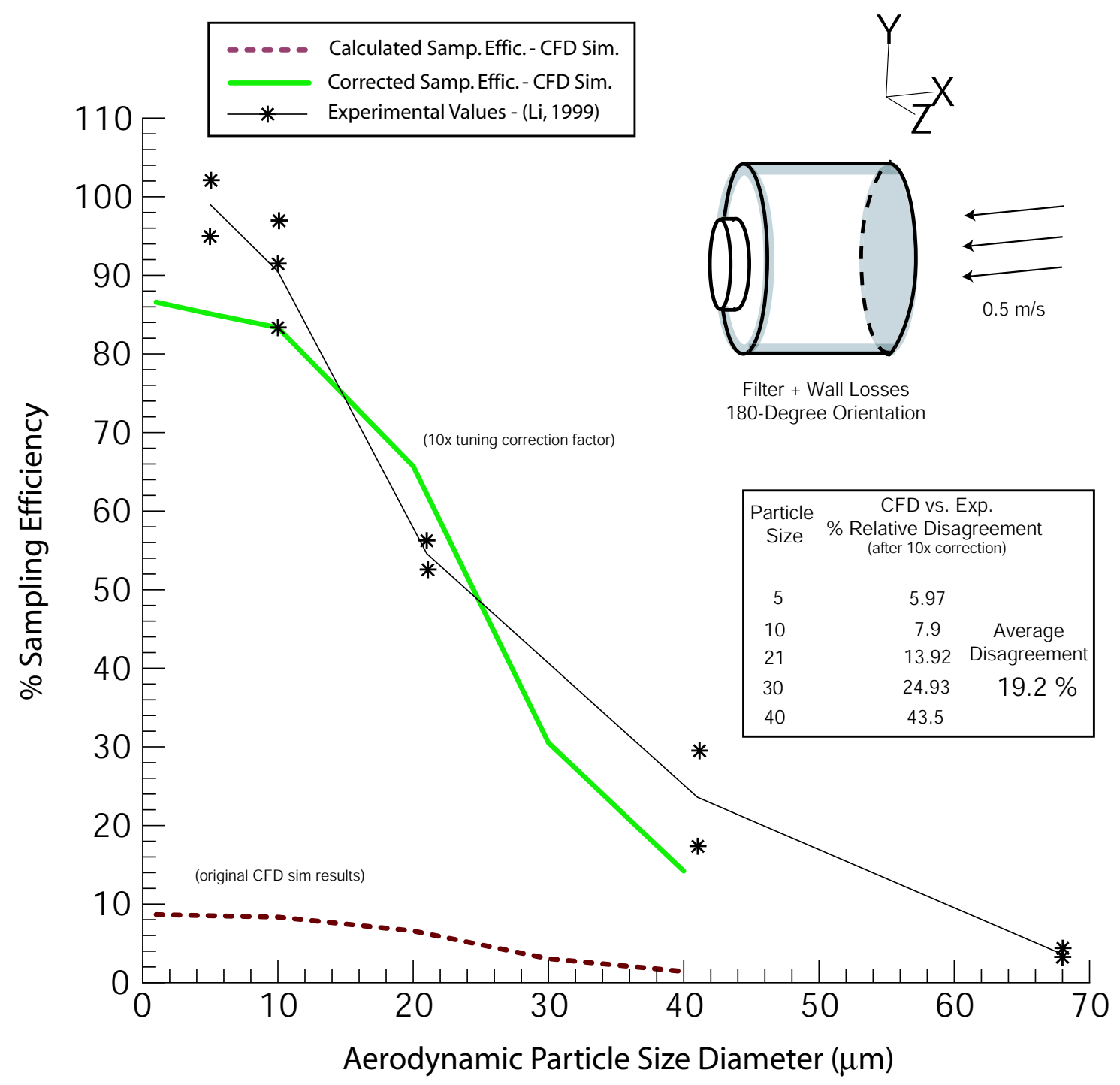

Figure 4.33: Sampling efficiencies of IOM sampler in comparison with experimental results from Li (1999) for the sampler free in the air and oriented 180 degrees to a $0.5 \mathrm{~m} / \mathrm{s}$ freestream airflow. 


\section{Direction-Specific Results: 1.0 m/s Freestream Windspeed}

Results of tuned, direction-specific sampling efficiencies are plotted with experimental values in Figures 4.34, 4.35, and 4.36 for orientations of 0-, 90-, and 180-degrees to $1.0 \mathrm{~m} / \mathrm{s}$ freestream airflow. Correction tuning constants were applied as described in the previous section for the GSP sampler and are shown in Table 4.9. Agreement with the Li experimental data are reasonable for most physical scenarios. However, for larger particles at the 0-degree orientation, CFD significantly underpredicts the sampling efficiency of the IOM sampler. 


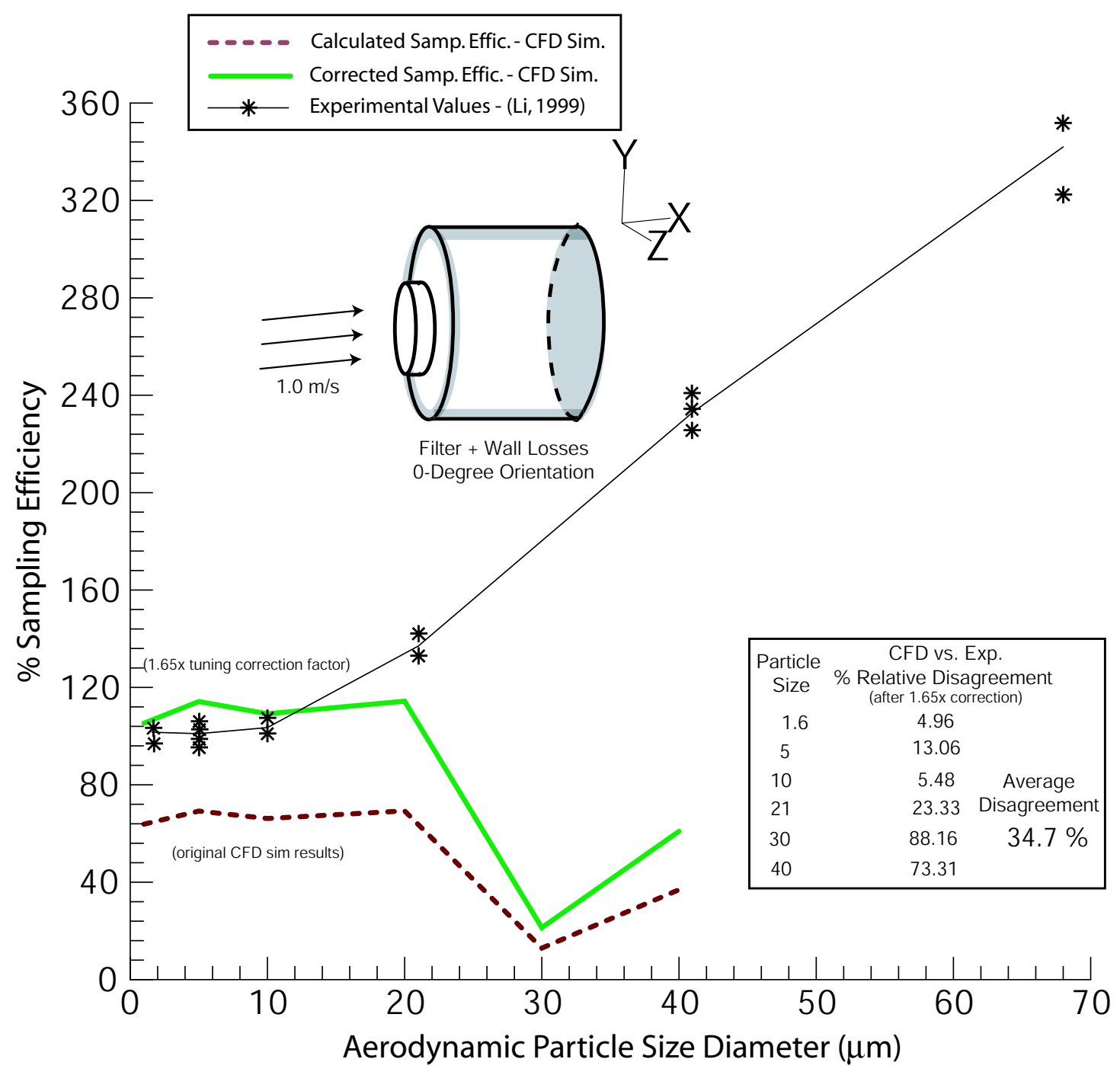

Figure 4.34: Sampling efficiencies of IOM sampler in comparison with experimental results from $\mathrm{Li}$ (1999) for the sampler free in the air and oriented 0 degrees to a $1.0 \mathrm{~m} / \mathrm{s}$ freestream airflow. 


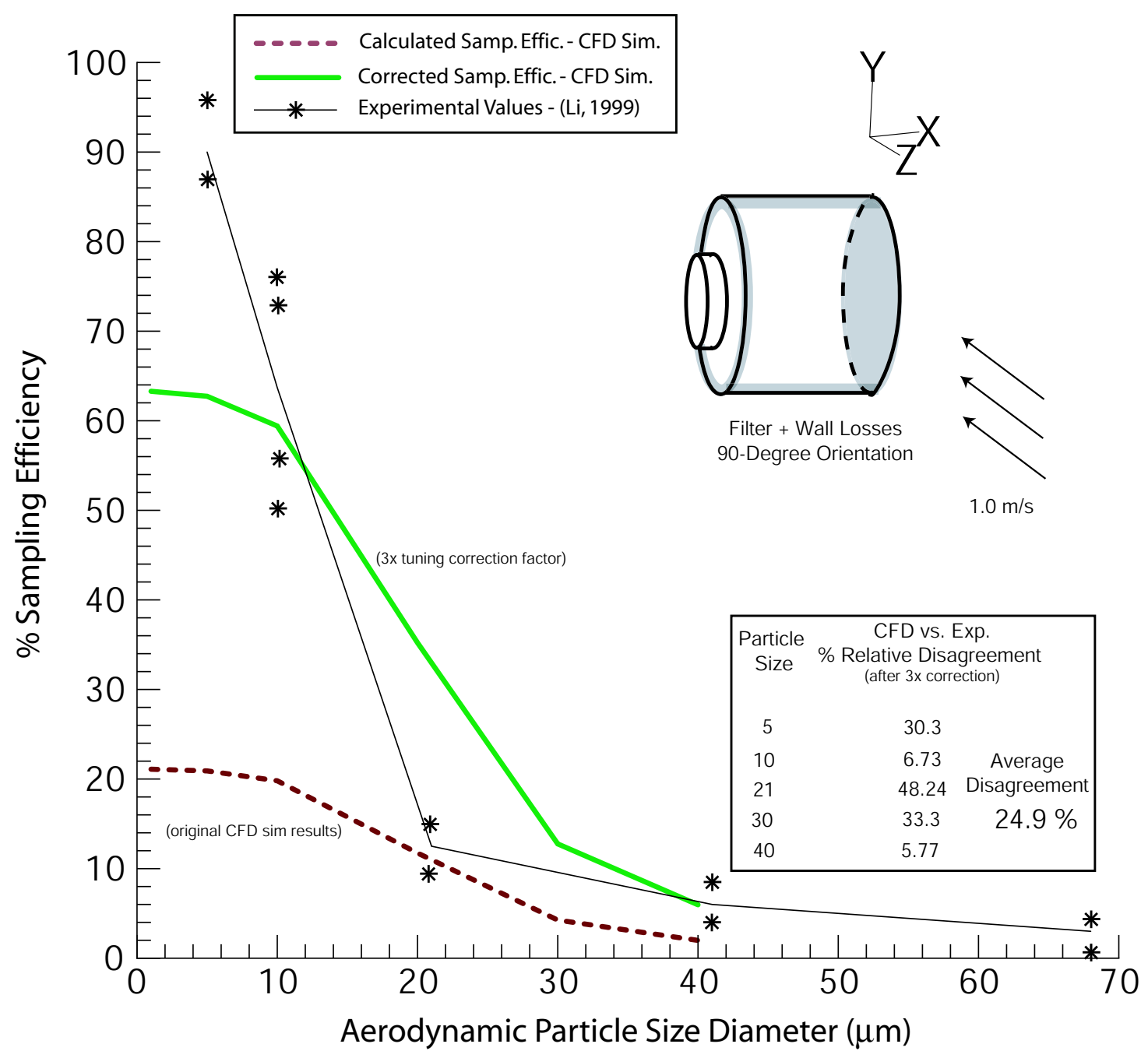

Figure 4.35: Sampling efficiencies of IOM sampler in comparison with experimental results from $\mathrm{Li}$ (1999) for the sampler free in the air and oriented 90 degrees to a $1.0 \mathrm{~m} / \mathrm{s}$ freestream airflow. 


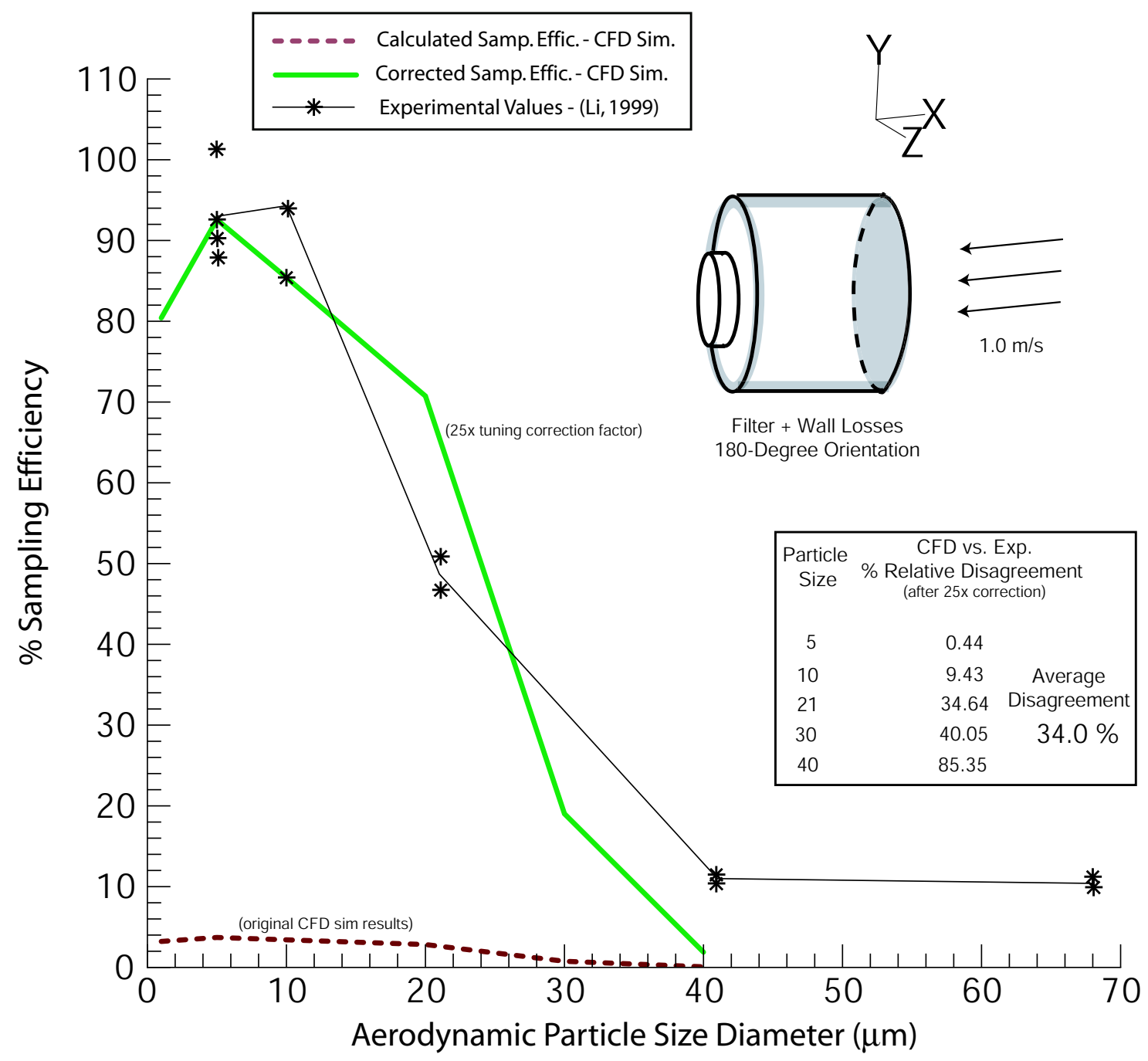

Figure 4.36: Sampling efficiencies of IOM sampler in comparison with experimental results from Li (1999) for the sampler free in the air and oriented 180 degrees to a $1.0 \mathrm{~m} / \mathrm{s}$ freestream airflow. 
Table 4.8: Correction tuning factors based on experimental values from Li (1999) and the resulting average percent relative disagreement for that condition.

\begin{tabular}{|c|c|c|c|}
\hline Orientation & Windspeed & $\begin{array}{c}\text { Tuning Factor } \\
\text { Filter S.E. }\end{array}$ & $\begin{array}{l}\text { Avg. \% Rel. } \\
\text { Disagreement }\end{array}$ \\
\hline 0 & 0.5 & 1.9 & 26.5 \\
\hline 0 & 1.0 & 1.65 & 34.7 \\
\hline 90 & 0.5 & 4.0 & 37.8 \\
\hline 90 & 1.0 & 3.0 & 24.9 \\
\hline 180 & 0.5 & 10.0 & 19.2 \\
\hline 180 & 1.0 & 25.0 & 34.0 \\
\hline
\end{tabular}


Table 4.9: Average correction tuning factors for 0-, 90-, and 180-degree orientations to freestream flows of 0.5 and $1.0 \mathrm{~m} / \mathrm{s}$ for IOM sampler with deviation from original values of relative percent disagreement in parentheses.

\begin{tabular}{|c|c|c|c|}
\hline & & $\begin{array}{c}\text { Tuning Factor } \\
\text { Orientation }\end{array}$ & Wing. \% Rel. \\
0 & 0.5 & 1.775 & $12.24(-14.28)$ \\
0 & 1.0 & 1.775 & $31.98(-2.74)$ \\
90 & 0.5 & 3.5 & $50.29(+12.5)$ \\
90 & 1.0 & 3.5 & $54.64(+29.78)$ \\
180 & 0.5 & 17.5 & $48.38(+29.14)$ \\
180 & 1.0 & 17.5 & $60.22(+26.24)$ \\
& & & \\
\hline
\end{tabular}


As with the GSP sampler, common tuning factors were identified by averaging the factors that were within $+/-25 \%$. Table 4.9 shows the results of averaging tuning factors by wind speed. For the 0-degree orientation there is a net effect of decreasing the overall level of disagreement for both cases. However at the 90- and 180-degree orientations, averaging results in greater disagreement.

\section{IOM Mounted on Box-Shaped Bluff Body}

CFD simulations and particle trajectory calculations were conducted the same as the GSP for $0.5,1.25$, and $2.0 \mathrm{~m} / \mathrm{s}$ freestream velocities. Again, simulation results were compared to the directionally-averaged, experimental data collected by Kenny et al. (1997), and sampling efficiency was determined in reference to the ideal sampling probe. The Witschger average (Equation 4.1, Figure 4.37) and the simple directional average (Equation 4.2, Figure 4.38) were both used to determine the final sampling efficiency values for the 0.5 $\mathrm{m} / \mathrm{s}$ freestream wind speed. Calculated sampling efficiency values from 1.25 and $2.0 \mathrm{~m} / \mathrm{s}$ freestream velocities were plotted together with experimental sampling efficiency values measured in wind speeds of 1.0 and $4.0 \mathrm{~m} / \mathrm{s}$ freestream airflows (Figure 4.39). Agreement at the 0.5 and $1.25 \mathrm{~m} / \mathrm{s}$ wind speeds was reasonable, but there was significant disagreement for the larger particle size at the $2.0 \mathrm{~m} / \mathrm{s}$ freestream airflow wind speed. Sampling efficiency results from CFD were tuned based on discrete values from the Inhalability convention, and the tuning correction factors are shown in Table 4.10. Differences of individual corrections values were too large to use common tuning correction factors as was done previously. 


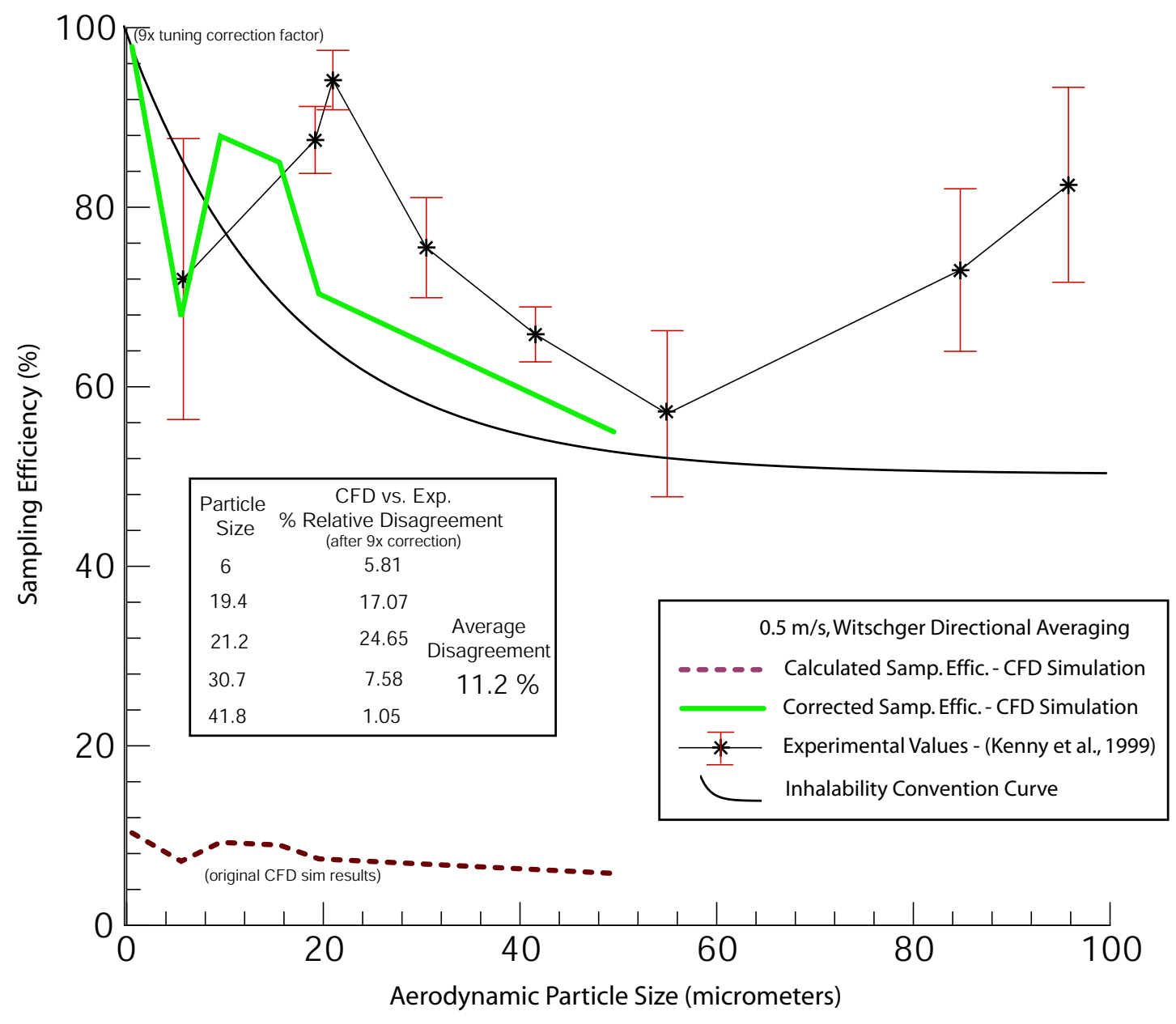

Figure 4.37: Witschger direction-averaged sampling efficiency values for IOM sampler mounted on box-shaped bluff body in freestream windspeed of $0.5 \mathrm{~m} / \mathrm{s}$. with experimental values from Kenny et al. (1997) 


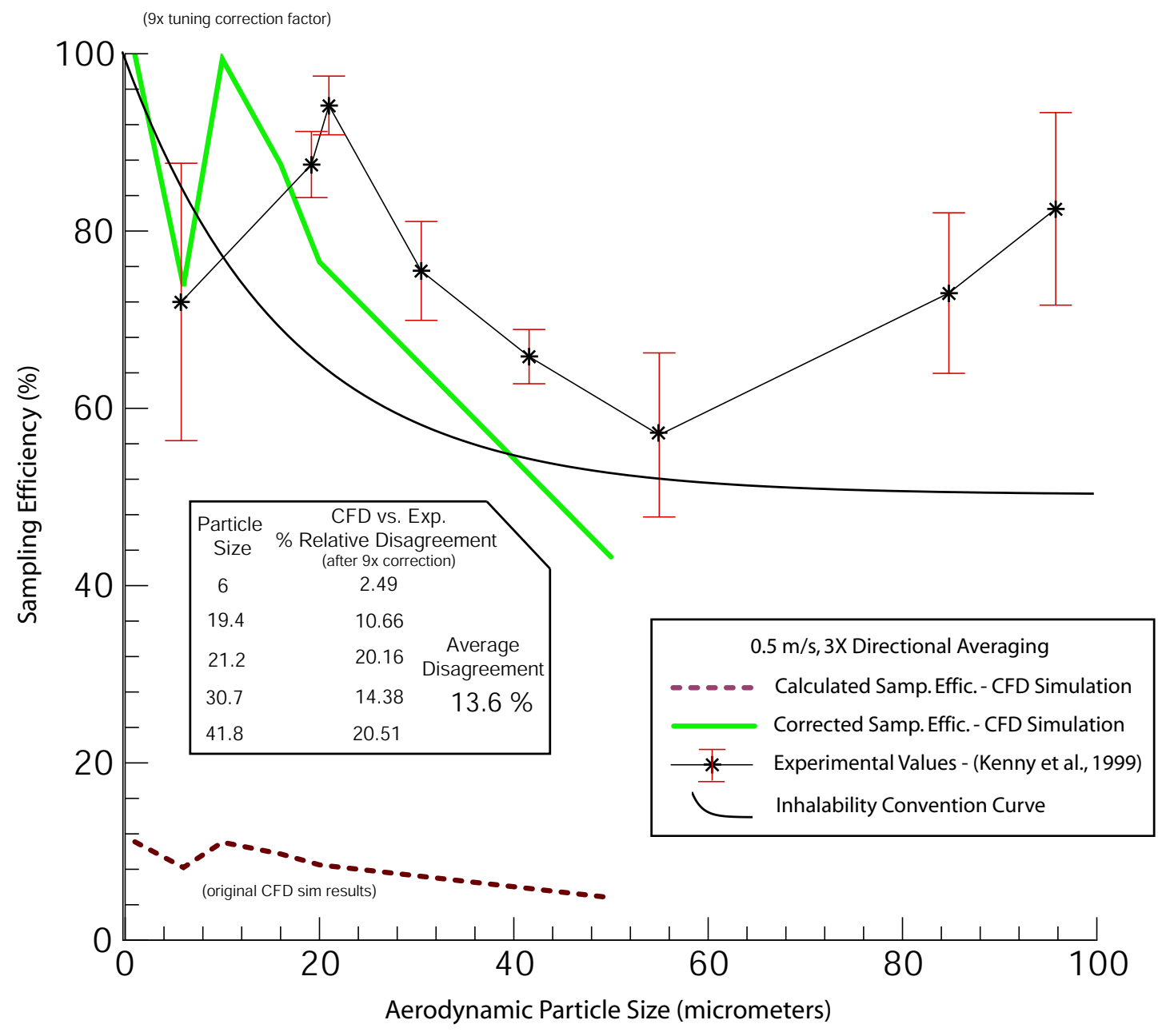

Figure 4.38: $3 X$ direction-averaged sampling efficiency values for IOM sampler mounted on box-shaped bluff body in freestream windspeed of $0.5 \mathrm{~m} / \mathrm{s}$. with experimental values from Kenny et al. (1997) 


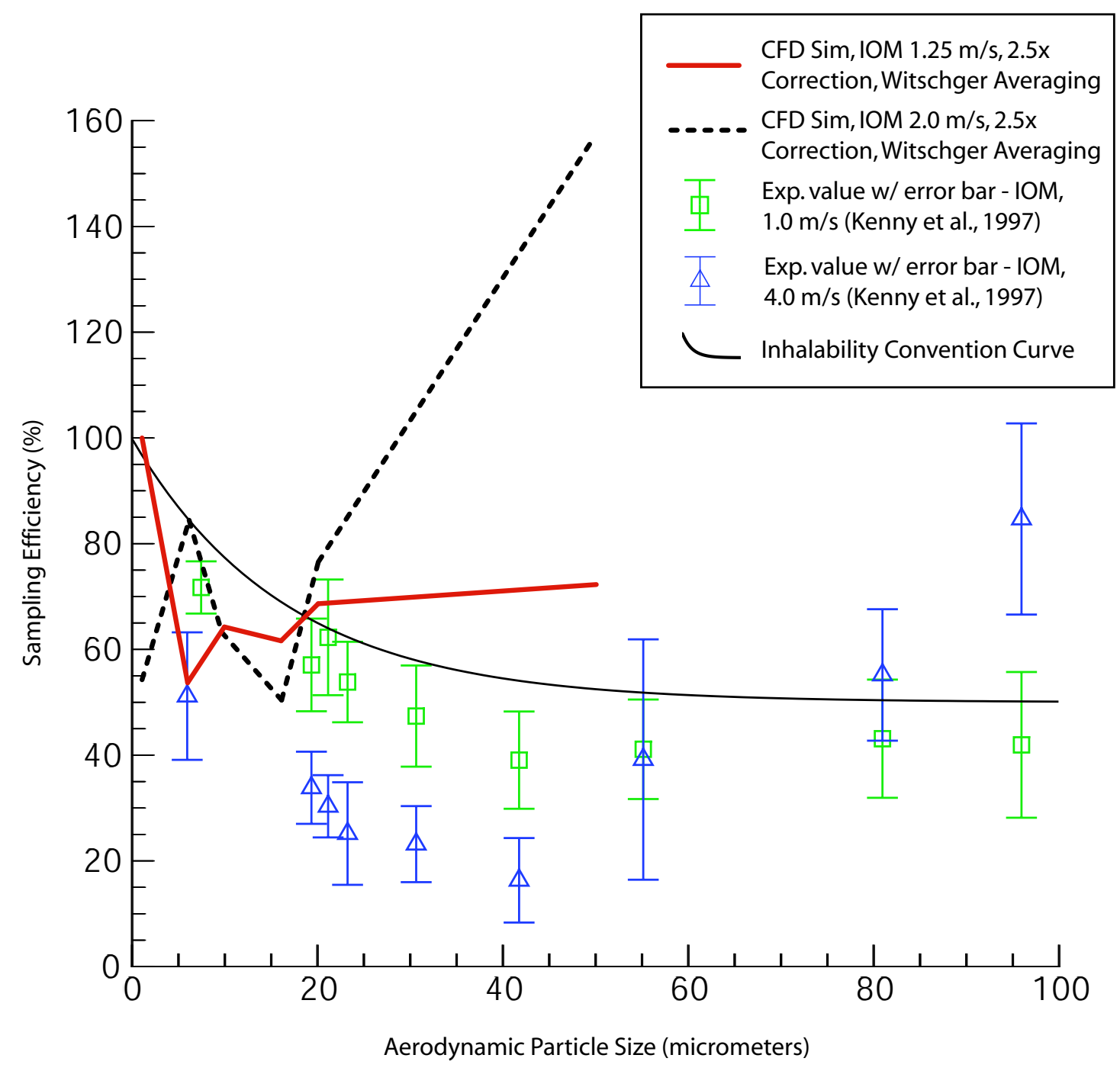

Figure 4.39: Witschger direction-averaged sampling efficiency values for the IOM sampler mounted on a box-shaped bluff body in freestream windspeeds of 1.25 and $2.0 \mathrm{~m} / \mathrm{s}$ with experimental values from Kenny et al. (1997) for windspeeds of 1.0 and $4.0 \mathrm{~m} / \mathrm{s}$. 
Table 4.10: Correction tuning constants, based on Inhalability convention, and the operation function on their respective data sets from simulations of the IOM sampler mounted on the box-shaped bluff body.

\begin{tabular}{|c|c|c|c|}
\hline & $\begin{array}{c}\text { Tuning Factor } \\
\text { Windspeed }\end{array}$ & $\begin{array}{c}\text { Tuning Factor } \\
\text { 3X Avg. }\end{array}$ & \\
0.5 & 9 & 9 & Multschger Avg. \\
1.25 & 20 & 20 & Function \\
2.0 & 30 & 30 & Multiplied \\
& & & \\
\hline
\end{tabular}




\section{Chapter 5}

\section{Discussion and Conclusions}

\subsection{Overview}

Sampling efficiency values of the GSP and IOM Inhalable personal aerosol samplers were calculated using a commercially available CFD software package for a range of particle sizes and wind speeds. Particle trajectories were calculated based on airflow simulations obtained from independent grids of the GSP and IOM samplers free in the air and mounted on a box-shaped bluff body.

Initially, results of simulations were found to disagree with those from experiments. However, the CFD results were of the same magnitude and had similar trends as the experimental results, especially for particle sizes from 1 to $40 \mu \mathrm{m}$ in wind speeds of 0.5 and 1.0 $\mathrm{m} / \mathrm{s}$. Therefore, exploratory correction factors were applied to the CFD results to learn if 
agreement could be obtained by adjusting the CFD results up or down, depending on their values with respect to experiment.

A single correction factor was used for each physical scenario, so that adjustment of the CFD results did not replace simulation as a modeling method. This attempt was met, generally, with success, although in some cases there were calculated sampling efficiency values that did not agree with experimental results, even after correction. It is believed that one reason for the disagreement in those cases is due to use of a "trial-and-error" approach for determination of ideal sampling faces and numbers of particles released.

Correction factors were applied on a simulation-by-simulation basis, as experimental data were available. In the text, this is referred to as "tuning" of the CFD simulation results to experimental results. Since the simulations were compared to experiments of similar physical scenarios, the tuning correction factors were usually different from one another. In some cases, there was little difference, but in others, the differences were large. For the cases where tuning correction factors were reasonably close, a common correction factor was sought that could be applied to multiple physical scenarios. This was done because use of common correction factors will reduce the total number of experiments required to evaluate the performance of the aerosol sampler in question.

Common correction factors were obtained for the following: (1) the GSP sampler free in the air at a 0 -degree orientation for both 0.5 and $1.0 \mathrm{~m} / \mathrm{s}$ freestream wind speeds; (2) the GSP sampler mounted on a box-shaped bluff body using Witschger direction averaging for wind speeds of $0.5,1.25$, and $2.0 \mathrm{~m} / \mathrm{s}$; and (3) the IOM sampler free in the air at a 0 -degree 
orientation for both 0.5 and $1.0 \mathrm{~m} / \mathrm{s}$ freestream wind speeds.

\subsection{Discussion}

The CFD simulations conducted in this work were dependent on several key components: properly operating computers, existence of experimental data for validating and calibrating calculations, grid-independent solutions, availability of a verified turbulence model, and identification of ideal particle release groupings in the computational domain. Of these, the grid-independent solutions and identification of particle release groupings were the most critical in this work and deserve further discussion.

\section{Grid-Independent Solutions}

Grid-independent solutions involved parametric studies of different grid sizings both within the domain and near the walls in order to identify an adequate mesh resolution that would result in reasonable values. Adequate mesh resolution was initially examined through a trial-and-error process in order to reduce the total number of possibilities and begin to focus on those that were within the realm of acceptability. This required a lengthy investigation with focus on the mesh size (and type) at critical areas of calculation, specifically the surfaces and inlets of the computational versions of the samplers.

This process went hand-in-hand with application of turbulence models because of the importance of maintaining certain mesh sizes near walls and surfaces. For example, exces- 
sively fine grids were found to be unnecessary, particularly when enhanced wall treatments and wall functions were used. Use of the RNG k-e enhanced wall treatment enabled use of a coarser grid, which reduced computational time. However, there is a maximum size that will give reasonably correct solutions, and thus it was important to approach, but not exceed this critical mesh size.

In this work, it was found that the critical maximum was a function of the size of the mesh elements within the geometries and the 32-bit memory limitations of the computers used for calculations. In the case of the GSP sampler, the physical size of the leading edge of the GSP sampler $(0.0005 \mathrm{~m})$ was the minimum size that mesh elements could have in the inlet of the sampler and still result in total mesh size less than the maximum allowed by a 32-bit computer, which is around 5 million mesh elements, given a growth factor of around 105 to $110 \%$ from cell to cell. The IOM Inhalable sampler, on the other hand, has a leading edge that is on the order of $0.0001 \mathrm{~m}$ in size, which is well below the minimum size that will allow a total number of mesh elements less than 5 million. Thus, simulations were not possible for the leading edge of the IOM inlet. Instead, mesh was created to encompass the inlet of the IOM sampler as an exit from the domain, rather than attempt to calculate the interior flow features of the sampler as was the case with the GSP. This was consistent with how the samplers are used: the sampling efficiency of the IOM sampler is determined from all particles that enter the inlet, while the GSP sampling efficiency is determined only from filter deposition.

Grids were based on starting cell sizes of $0.0005 \mathrm{~m}$ and increased to $0.0010,0.0015$, and 
$0.0020 \mathrm{~m}$. At the largest cell size of 0.0020 , mesh resolution on the surfaces and walls of the computational domains resulted in incorrect values of wall $y^{+}$, which necessitated grid adaptation to the size of 0.0010 in order to achieve correct values. In flows away from the walls, all of the grid sizes gave similar values of velocity magnitude (as a vector of comparison) although grid convergence index studies showed the sizes to give slightly different results. Given the grid-convergence index results and what the computers could handle, it was found that the grid cell size of $0.0010 \mathrm{~m}$ was the 'engineering solution' of cell size in the computational inlets for all simulations in this class of flows. This result was confirmed with reasonable agreement between calculated and experimental sampling efficiency values.

\section{Particle Release Groupings}

Calculated sampling efficiency was found, through trial-and-error, to be dependent on the number and location of particles released into the computational domain. Thus it was critical to identify the locations from where particles should be released and keep the total number above the minimum threshold, which was estimated at around 30,000 particles. Particle release numbers were kept at numbers of at least 50,000, and often over 100,000 and these values resulted in repeatable sampling efficiency values.

Release of particles from multiple locations and faces results in the best distribution. Good results were found with release of at least 20,000 particles per grouping (total of 100,000). Release locations do have an effect on resulting sampling efficiency values, 
and must be calibrated for testing samplers. This was done by using a sharp-edged, thinwalled sampler as defined by Belyaev and Levin (1974) and then iteratively running various combinations of release faces and initial concentrations until the freestream "true" sampler faces agree with the Belyaev and Levin sampler. Once this occurred, inlets (of the GSP and IOM) were evaluated at an X location equal to that of inlet of the sharp-edged, thin-walled sampler.

The total number of particles released was broken up into five release groupings per sampler, which resulted in ten per simulation since there was both a test sampler (GSP or IOM) and a reference probe in each computational domain. These groupings could be released over 360 degrees so there was a uniform particle distribution at the point of entry into the two ideal sampling faces, as well as for the test sampler and reference probe. For particle sizes up to $40 \mu \mathrm{m}$, in this work, it was possible to attain uniform distribution. However, for larger particles it was not. It is expected that larger particles could also be uniformly distributed if the correct combination of release groupings is found. It may, in fact, be necessary to use different release groupings for particle sizes larger than $40 \mu \mathrm{m}$ from those for particle sizes less than $40 \mu \mathrm{m}$. In addition, use of the parameter space analysis method in a more rigorous parametric and factorial-like approach may allow a more quantitative determination of where and how many particles should be released. This could be addressed in future work. 


\section{Chapter 6}

\section{References}

Addlesee, A. J. 1980. Anisokinetic Sampling of Aerosols at a Slot Intake. Journal of Aerosol Science. Vol. 11. pp. 483-493.

Aitken, R. J., Baldwin, P. E. J., Beaumont, G.C., Kenny, L.C., Maynard, A.D. 1999. Aerosol Inhalability in Low Air Movement Environments. Journal of Aerosol Science. Vol. 30, No. 5. pp. 613-626.

Aizenberg, V., Grinshpun, S. A., Willeke, K., Smith, J., Baron, P. A. 2000. Performance Characteristics of the Button Personal Inhalable Aerosol Sampler. American Industrial Hygiene Association Journal. Vol. 61. pp. 398-404.

Aizenberg, V., Grinshpun, S. A., Willeke, K., Smith, J., Baron, P. A. 2000. Measurement of the Sampling Efficiency of Personal Inhalable Aerosol Samplers Using a Simplified Protocol. Journal of Aerosol Science. Vol. 31, No. 2, pp. 169-179.

Armbruster, L. and Breuer, H. 1982. Investigations Into Defining Inhalable Dust. Annals of Occupational Hygiene. Vol. 26, pp. 21. 
Armbruster, L. and Zebel, G. 1985. Theoretical and Experimental Studies for Determining the Aerosol Sampling Efficiency of Annular Slot Probes. Journal of Aerosol Science. Vol. 16, No. 4. pp. 335-341.

Badzioch, S. 1959. Collection of Gas-Borne Dust Particles by Means of an Aspirated Sampling Nozzle. British Journal of Applied Physics. Vol. 10, pp 26-32.

Badzioch, S. 1960. Correction for Anisokinetic Sampling of Gas-borne Dust Particles. Journal of the Institute of Fuel. Vol. 33, pp 106-110.

Baldwin, P. E. J. and Maynard, A. D. 1998. A Survey of Wind Speeds in Indoor Workplaces. Annals of Occupational Hygiene. Vol. 42, No. 5, pp. 303-313.

Baron, Paul A., Chen, C. -C., Hemenway, D. R, O'Shaughnessy, P. 1994. Nonuniform Air Flow In Inlets: The Effect on Filter Deposits in the Fiber Sampling Cassette. American Industrial Hygiene Association Journal. Vol. 55, pp. 722-732.

Belyaev, S. P., Levin, L. M. 1972. Investigation of Aerosol Aspiration by Photographing Particle Tracks Under Flash Illumination. Aerosol Science* (Journal of). Vol. 3. pp. 127140. (* In 1973, Aerosol Science was called Journal of Aerosol Science.)

Belyaev, S. P., Levin, L. M. 1974. Techniques for Collection of Representative Aerosol Samples. Journal of Aerosol Science. Vol. 5. pp. 325-338.

Berges, Markus. 2004. Berufsgenossenschaftliches Institut fr Arbeitsschutz - BIA (im HVBG) BG-Institute for Occupational Safety and Health (of HVBG)) Fachbereich Gefahrstoffe: Umgang - Schutzmanahmen Referat Expositionsbewertung Alte Heerstrae 11153757 Sankt Augustin Germany. Personal Communication.

Brady, W., Touzalin, L. A. 1911. The Determination of Dust Blast-Furnace Gas. The Journal of Industrial and Engineering Chemistry. Sept. 1911, pp. 662-670. 
Breslin, J. A. and Stein, R. L. 1975. Efficiency of Dust Sampling Inlets in Calm Air. American Industrial Hygiene Association Journal. Vol. 36, pp. 576-583.

Chen, C. -C., Baron, P. A. 1995. Vortices in Anisoaxial Sampling Inlets. Aerosol Science and Technology. Vol. 23. pp. 224-230.

Chen, C. -C. and Baron, P. A. 1996. Aspiration Efficiency and Inlet Wall Deposition in the Fiber Sampling Cassette. Am. Ind. Hyg. Journal. Vol. 57. pp. 142-152.

Chen, C. -C., Lai, C. -Y., Shih, T. -S., Yeh, W. -Y. 1998. Development of Respirable Aerosol Samplers Using Porous Foams. American Industrial Hygiene Association Journal. Vol. 59. pp. 766-773.

Choudhury, D. 1993. Calculation of Turbulent Separated Flows Using a Renormalization Group Based k - e Turbulence Model. ASME Fluids Engr. Conf. March 2, 1993.

Chung, K. Y. K., Ogden, T. L., Vaughn, N. P. 1987. Wind Effects on Personal Dust Samplers. Journal of Aerosol Science. Vol. 18, No. 2, pp. 159-174.

Chung, I. P. and Dunn-Rankin, D. 1992. Numerical Simulation of Two-Dimensional Blunt Body Sampling in Viscous-Flow. Journal of Aerosol Science. Vol. 23, No.3. pp. 217-232.

Comite' Europeen de Normalisation (CEN). 1992. Workplace atmospheres: size fraction definitions for measurement of airborne particles in the workplace, CEN Standard EN 481.

Davies, C. N. 1947. The Sedimentation of Small Suspended Particles. Supplement to Transactions, Institute of Chemical Engineers. Vol. 25, pp. 25-39.

Davies, C. N. 1968. The Entry of Aerosols into Sampling Tubes and Heads. British Journal of Applied Physics. Vol. 1, No. 7, pp. 921-932. 
Davies, C. N. and Subari, M. 1978. Inertia Effects in Sampling Aerosols. Proceedings: Advances in Particle Sampling and Measurement. Ashville, N.C., May 1978. pp. 1-29.

Davies, C. N. and Subari, M. 1978. Aspiration above Wind Velocity of Aerosols with Thin-Walled Nozzles Facing and at Right Angles to the Wind Direction.Journal of Aerosol Science. Vol. 13, No. 1, pp. 59-71.

Dunnett, S. J. and Ingham, D. B. A Mathematical Theory for Two-Dimensional Blunt Body Sampling. Journal of Aerosol Science. Vol. 17, No. 5, pp. 839-853.

Dunnett, S. J. 1999. An Analytical Investigation into the Nature of the Airflow near a Spherical Bluff Body with Suction. Journal of Aerosol Science. Vol. 30, No. 2. pp. 163 171.

Dunnett, S. J. and Vincent, J. H. 2000. A Mathematical Study of Aerosol Sampling by an Idealised Blunt Sampler Oriented at an Angle to the Wind: The Role of Gravity. Journal of Aerosol Science. Vol. 31, No. 10 pp. 1187-1203.

Durham, M. D., Lundgren, D. A. 1980. Evaluation of Aerosol Aspiration Efficiency as a Function of Stokes Number, Velocity Ratio and Nozzle Angle. Journal of Aerosol Science. Vol. 11. pp. 179-188.

Feather, G. and Chen, B. T. 2003 Design and Use of a Settling Chamber for Sampler Evaluation under Calm-Air Conditions. Aerosol Science and Technology. Vol. 37, pp., 261-270.

Ferziger, J. H. and Peric', M. 1999. Computational Methods for Fluid Dynamics. SpringerVerlag, New York.

Fluent Inc. 2003. FLUENT 6 User's Guide. Fluent Inc. Lebanon, NH. 
Flynn, M. R. and Miller, C. T. 1991. Discrete Vortex Methods for the Simulation of Boundary Layer Effects on Worker Exposure. Annals of Occupational Hygiene. Vol. 35, No. 1. pp. 35-40.

Flynn, M. R., Chen, M. -M., Taehyeung, K., and Muthedath, P. 1995. Computational Simulation of Worker Exposure Using a Particle Trajectory Method. Annals of Occupational Hygiene. Vol. 39, No. 3. pp. 277-289.

Flynn, M. R., Lackey, B. D., Muthedath, P. 1996. Experimental and Numerical Studies on the Impact of Work Practices Used to Control Exposures Occurring in Booth- Type Hoods. American Industrial Hygiene Association Journal. Vol. 57. pp. 469- 475.

Flynn, M. R., Gatano, B. L., McKernan, J. L., Dunn, K. H., Bazicko, B. A., Carlton, G. N. 1999. Modeling Breathing-Zone Concentrations of Airborne Contaminants Generated During Compressed Air Spray Painting. Annals of Occupational Hygiene. Vol. 43, No. 1, pp. 67-76.

Flynn, M. R. and Sills, E. D. 2000. On the Use of Computational Fluid Dynamics in the Prediction and Control of Exposure to Airborne Contaminants - An Illustration Using Spray Painting. Annals of Occupational Hygiene. Vol. 44, No. 3, pp. 191-202.

Flynn, M. R. and Sills, E. D. 2001. Numerical Simulation of Human Exposure to Aerosols Generated During Compressed Air Spray-Painting in Cross-Flow Ventilated Booths. Journal of Fluids Engineering. Vol. 123. pp. 64-70.

Fuchs, N. A. 1975. Review Papers: Sampling of Aerosols. Atmospheric Environment. Vol. 9. Pp. 697-707.

Gao, P., Dillon, H. K., Farthing, W. E. 1997. Development and Evaluation of an Inhalable Bioaerosol Manifold Sampler. American Industrial Hygiene Association Journal. Vol. 58. pp. 196-206. 
Gao, P., Dillon, H. K., Baker, J., Oestenstad, K. 1999. Numerical Prediction of the Performance of a Manifold Sampler with a Circular Slit Inlet in Turbulent Flow. Journal of Aerosol Science. Vol. 30, No 3. pp. 299-312.

Gao, P.; Chen, B. T.; Baron, P. A.; Soderholm, S. C. 2002. A Numerical Study of the Performance of an Aerosol Sampler with a Curved, Blunt, Multi-Orificed Inlet. Aerosol Science and Technology. Vol. 30, No 3. pp. 299-312.

Gautam, M., Sreenath, A. 1997. Performance of a Respirable Multi-Inlet Cyclone Sampler. Journal of Aerosol Science. Vol. 28, No. 7. pp. 1265-1281.

Gibson, H. and Ogden, T. L. 1977. Some Entry Efficiencies for Sharp-Edged Samplers in Calm Air. Journal of Aerosol Science. Vol. 8, pp. 361-365.

Gong, H., Anand, N. K., McFarland, A. R. 1993. Numerical Prediction of the Performance of a Shrouded Probe Sampling in Turbulent Flow. Aerosol Science and Technology. Vol. 19. pp. 294-304.

Griffiths, J. H. and Jones, T. D. 1939. The Determination of Dust-Concentrations in Mine Atmospheres. Transactions- The Institution of Mining Engineers. Vol. 99. pp. 150-180.

Griffiths, W. D. and Boysan, F. 1992. An Assessment of the Application of Computational Fluid Dynamics (CFD) to Model the Performance of a Range of Small Sampling Cyclones. Journal of Aerosol Science. 23, Suppl. 1. pp. S587-S590.

Griffiths, W. D. and Boysan, F. 1996. Computational Fluid Dynamics (CFD) and Empirical Modelling of the Performance of a Number of Cyclone Samplers. Journal of Aerosol Science. Vol. 27, No. 2. pp. 281-304.

Grinshpun, S., Willeke, K., Kalatoor, S. 1993. A General Equation for Aerosol Aspiration by Thin-Walled Sampling Probes in Calm and Moving Air. Atmospheric Environment. Vol. 27A, No.9. pp. 1459-1470. 
Hangal, S. and Willeke, K. 1990. Aspiration Efficiency: Unified Model for All Forward Sampling Angles. Environmental Science and Technology. Vol. 24, No. 5. pp. 688-691.

Hangal, S. and Willeke, K. 1992. Aerosol Sampling at Small Forward-Facing Angles: Differentiation of Yaw from Pitch. Atmospheric Environment. Vol. 26A, No. 16. pp. 2913-2921.

Harper, M. 2004. Personal communications over photographs of aerosol particulate on filters collected in lead-smelting foundries.

Hauck, B. C., Grinshpun, S. A., Reponen, A., Reponen, T., Willeke, K., Bornschein, R. L. 1997. Field Testing of a New Aerosol Sampling Method with a Porous Curved Surface as Inlet. American Industrial Hygiene Association Journal. Vol. 58. pp. 713-719.

Heyder, J. and Gebhart, J. 1977. Gravitational Deposition of Particles from Laminar Aerosol Flow Through Inclined Circular Tubes. Journal of Aerosol Science. Vol. 8. pp. 289-295.

Hinds, W. C. 1999. Aerosol Technology, Properties, Behavior, and Measurement of Airborne Particles. 2nd ed. John Wiley \& Sons, Inc.

Ingham, D. B. 1981. The Entrance of Airborne Particles into a Blunt Sampling Head. Journal of Aerosol Science. Vol. 12, No. 6, pp. 541-549.

International Organization for Standardization. 1995. Guide to the Expression of Uncertainty in Measurement. 1st Ed.

JFE - Journal of Fluids Engineering. 2004. JFE Publication Policy, subpart b, Recommended procedure for estimation of discretization error. Online at http://www.asme.org/divisions/fed/divisionadmin/cfdindex.html 
Kalatoor, S., Grinshpun, S. A., Willeke, K. 1995. Aerosol Sampling from Fluctuating Flows into Sharp-Edged Tubular Inlets. Journal of Aerosol Science. Vol. 26, No.3. pp. 387-398.

Kalatoor, S., Grinshpun, S. A., Willeke, K., Baron, P. 1995. New Aerosol Sampler With Low Wind Sensitivity and Good Filter Collection Uniformity. Atmospheric Environment. Vol. 29, No. 10. pp. 1105-1112.

Kennedy, E. R., Fischbach, T. J., Song, R., Eller, P. M., Shulman, S. A. 1995. Guidelines for Air Sampling and Analytical Method Development and Evaluation. DHHS (NIOSH) Number 95-117.

Kennedy, N. J., Tatyan, K., Hinds, W. C. 2001. Comparison of a Simplified and FullSize Mannequin for the Evaluation of Inhalable Sampler Performance. Aerosol Science and Technology. Vol. 35, pp. 564-568.

Kenny, L. C., Aitken, R., Chalmers, C., Fabries, J. F., Fernandez, E. Gonzalez, Kromhout, H., Liden, G., Mark, D., Riediger, G., Prodi, V. 1997. A Collaborative European Study of Personal Inhalable Aerosol Sampler Performance. Annals of Occupational Hygiene. Vol. 41, No. 2. pp. 135-153.

Kenny, L. C., Aitken, R. J., Baldwin, P. E. J., Beaumont, G.C., Maynard, A.D. 1999. The Sampling Efficiency of Personal Inhalable Aerosol Samplers in Low Air Movement Environments. Journal of Aerosol Science. Vol. 30, No. 5. pp. 627- 638.

Li, J., Yavuz, I., Celik, I. B., Guffey, S. E., Bird, A. J. 2003. The Effect of Turbulence and Scalar Transport Models on Prediction of Worker Exposure to Aerosols. Proceedings of the Forum on CFD Applications in Large Facilities. 2003 ASME Fluids Engineering Division Summer Meeting. July 6-10, 2003.

Li, S. -H. and Lundgren, D. 2002. Aerosol Aspiration Efficiency of Blunt and Thinwalled Samplers at Different Wind Orientations. Aerosol Science and Technology. Vol. 36, pp. 342-350. 
Li, S. -H. 1999. Field and Laboratory Evaluation of Six Inhalable Aerosol Samplers and Two Impactors. Ph.D. Thesis, University of Florida, Gainesville, FL.

Liden, G., Juringe, L., Gudmundsson, A. 2000. Workplace Validation of a Laboratory Evaluation Test of Samplers for Inhalable and "Total" Dust. Journal of Aerosol Science. Vol. 31, No. 2 pp. 199-219.

Lipatov, G. N., Grinshpun, S. A., Semenyuk, T. I., Sutugin, A. G. 1988. Secondary Aspiration of Aerosol Particles into Thin-Walled Nozzles Facing the Wind. Atmospheric Environment. Vol. 22, No. 8. pp. 1721-1727.

Liu, B. Y. H., Zhang, Z. Q., Kuehn, T. H. 1989. A Numerical Study of Inertial Errors in Anisokinetic Sampling. Aerosol Science. Vol.20, No. 3. pp. 367-380.

Lo Savio, S., Paradisi, P., Tampieri, F., Belosi, F., Pia Morigi, M., and Agostini, S. 2003. Numerical Determination of Personal Aerosol Sampler Aspiration Efficiency. Applied Occupational and Environmental Hygiene. Vol. 18, No. 4, pp. 244-255.

Mark, D. and Vincent, J. H. 1986. A New Sampler for Airborne Total Dust in Workplaces. Annals of Occupational Hygiene. Vol. 30, No. 1, pp. 89-102.

Mauderly, J. L., Cheng, Y. S., Hoover, M. D., Yeh, H.-C. 2000. Particles Inhaled in the Occupational Setting, pp. 89-170, in Particle-Lung Interactions, edited by Peter Gehr and Joachim Heyder. Series Title: Lung Biology in Health and Disease, Vol. 143, Claude Lenfant, Executive Editor. Published by Marcel Dekker, New York. 
McClellan, R. O. 2000. Particle Interactions with the Respiratory Tract, pp. 3-63, in Particle-Lung Interactions, edited by Peter Gehr and Joachim Heyder. Series Title: Lung Biology in Health and Disease, Vol. 143, Claude Lenfant, Executive Editor. Published by Marcel Dekker, New York.

McFarland, A. R., Ortiz, C. A., Moore, M. E., DeOtte, R. E., Jr., Somasundaram, S. 1989. A Shrouded Aerosol Sampling Probe.Environmental Science and Technology. Vol. 23. pp. 1487-1492.

Millipore, Inc. 2004. Personal Communication with Lisa Phillips, Technical Service Specialist, to learn the history of the $37-\mathrm{mm}$ closed-face-cassette sampler.

Moore, G. E. 1965. Cramming More Components onto Integrated Circuits. Electronics. Vol. 38, No. 8 .

NIOSH. 2003. Work Related Lung Disease Surveillance Report 2002. DHHS (NIOSH) Number 2003-111.

Occupational Safety and Health Administration (OSHA). 2002. OSHA Technical Manual.

OSHA. 2004 ${ }^{a}$ Major Group 14: Mining And Quarrying Of Nonmetallic Minerals, Except Fuels.

OSHA. 2004 ${ }^{b}$. OSHA Chemical Sampling Information Silica (Quartz, Total), Revision Date 01/15/1993.

Ogden, T. L. and Birkett, J. L. 1977. The Human Head as a Dust Sampler. In Inhaled Particles. edited by W.H. Watson.

Ogden, T. L. and Birkett, J. L. 1978. An Inhalable Dust Sampler for Measuring the Hazard from Total Airborne Particulate. Annals of Occupational Hygiene. Vol. 21, pp. 41-50. 
Okazaki, K., Wiener, R. W., Willeke, K. 1987. Non-Isoaxial Aerosol Sampling: Mechanisms Controlling the Overall Sampling Efficiency. Environmental Science and Technology. Vol. 21, No. 2. pp. 183-187.

Patankar, S. 1980. Numerical Heat Transfer and Fluid Flow. Hemisphere Pub. Corp., McGraw-Hill, New York.

Pope, S. B. 2000. Turbulent Flows. Cambridge University Press, Cambridge, UK.

Puskar, M. A., Harkins, J. M., Moomey, J.D., Hecker, L. H. 1991. Internal Wall Losses of Pharmaceutical Dusts During Closed-Face, 37-mm Polystyrene Cassette Sampling. American Industrial Hygiene Association Journal. Vol 52, No 7. pp. 280-286.

Rao, N. P., Navascues, J., Fernandez De La Mora, J. 1993. Aerodynamic Focusing of Particles in Viscous Jets. Journal of Aerosol Science. Vol. 24, pp 879-892.

Richardson, L. F. 1911. The Approximate Arithmetical Solution by Finite Differences of Physical Problems Involving Differential Equations, with an Application to the Stresses in a Masonry Dam. Philosophical Transactions of the Royal Society of London. Vol. 210, Series A, pp. 307-357.

Roache, P. J. 1998. Verification and Validation in Computational Science and Engineering. Hermosa Publishers, Albuquerque, New Mexico.

Rodes, C. E., Kamens, R. M., Wiener, R. W. 1995. Experimental Considerations for the Study of Contaminant Dispersion Near the Body. American Industrial Hygiene Association Journal. Vol. 56. pp. 535-545.

Rose, V. E. 1997. History and Philosophy of Industrial Hygiene, in The Occupational Environment - Its Evaluation and Control. DiNardi, S., Editor. American Industrial Hygiene Association, Fairfax, VA, US. 
Smith, J. P., Bartley, D. L., Kennedy, E. R. 1998. Laboratory Investigation of the Mass Stability of Sampling Cassettes from Inhalable Aerosol Samplers. American Industrial Hygiene Association Journal). Vol. 59, pp. 582-585.

Smith, J. P. and Bird, A. J. 2002. Relationship of sampling efficiency for manikinmounted personal samplers to efficiency measurements made independent of manikin. Journal of Aerosol Science. Vol. 33, pp. 1235-1259.

Soderholm, S. 1989. Proposed International Conventions for Particle Size-Selective Sampling. Annals of Occupational Hygiene. Vol. 33, No. 3, pp. 301-320. Correction. 1991. Annals of Occupational Hygiene. Vol. 35, No. 3 pp. 357-358.

Soon Lan, N., and Viswanathan, S. 2001. Numerical Simulation of Airflow Around a Variable Volume/Constant Face Velocity Fume Cupboard. American Industrial Hygiene Association Journal. Vol. 62, pp. 303-312.

Sreenath, A., Ramachandran, G., Vincent, J. H. 1997. Experimental Investigations into the Nature of Airflows near Bluff Bodies with Aspiration, with Implications to Aerosol Sampling. Atmospheric Environment. Vol. 31, No. 15. pp. 2349-2359.

Tan, Y. M., Flynn, M. R., Buller, T. S. 2002. A Field Evaluation of the Impact of Transfer Efficiency on Worker Exposure During Spray Painting. Annals of Occupational Hygiene. Vol. 46, No. 1, pp. 103-112.

Taylor, G. I. 1935. Statistical Theory of Turbulence. Proceedings of the Royal Society of London. Series A, pp. 151.

Tennekes, H. and Lumley, J. L. 1972. A First Course in Turbulence. The MIT Press, Cambridge, MA, US, and London, England.

Torczynski, J. R. and Rader, D. J. 1997. The Virtual Cyclone: A Device for Nonimpact Particle Separation. Aerosol Science and Technology. Vol. 26. pp. 560-573. 
Tsai, C. -J. and Shih, T. -S. 1995. Particle Collection Efficiency of Two Personal Respirable Dust Samplers. American Industrial Hygiene Association Journal. Vol. 56. pp. 911-918.

Tsai, P. J., Vincent, J. H., Mark, D. 1996. Semi-Empirical Model for the Aspiration Efficiencies of Personal Aerosol Samplers of the Type Widely Used in Occupational Hygiene. Annals of Occupational Hygiene. Vol. 40, No. 1. pp. 93-113.

Tu, J. Y. 2000. Numerical Investigation of Particulate Flow Behavior in Particle-Wall Impaction. Aerosol Science and Technology. Vol. 32 pp. 509-526.

United States Code of Federal Regulations. 2004. 29 USC 651. Section 2, Congressional Findings and Purpose.

U.S. Dept. of Labor, Bureau of Labor Statistics. 2002. History of BLS Safety and Health Statistical Programs.

Versteeg, H. K., Malalasekera, W. 1995. An Introduction to Computational Fluid Dynamics. Prentice Hall, Essex, England.

Vincent, J. H. and Armbruster, L. 1981. On the Quantitative Definition of the Inhalability of Airborne Dust. Annals of Occupational Hygiene. Vol. 24, No. 2, pp. 245-248.

Vincent, J. H. and Gibson, H. 1981. Sampling Errors in Blunt Dust Samplers Arising from External Wall Loss Effects. Atmospheric Environment. Vol. 15, No. 5. pp. 703-712.

Vincent, J. H., Emmett, P. C., Mark, D. 1985. The Effects of Turbulence on the Entry of Airborne Particles into a Blunt Dust Sampler. Aerosol Science and Technology. Vol. 4. pp. 17-29. 
Vincent, J. H., Stevens, D. C., Mark, D., Marshall, M. and Smith, T. A. 1986. On the Aspiration Characteristics of Large-Diameter, Thin-Walled Aerosol Sampling Probes at Yaw Orientations with Respect to the Wind. Journal of Aerosol Science.. Vol. 18, No. 5, pp. 211-224.

Vincent, J. H. 1987. Recent Advances in Aspiration Theory for Thin-walled and Blunt Aerosol Sampling Probes. Journal of Aerosol Science.. Vol. 17, No. 2, pp. 487-498.

Vincent, J. H., Mark, D., Miller, B. G., Armbruster, L., and Ogden, T. L. 1990. Aerosol Inhalability at Higher Windspeeds. Journal of Aerosol Science. Vol. 21, No. 4, pp. $577-$ 586.

Vincent, J. H. 1994. Measurement of Coarse Aerosols in Workplaces. Analyst. Vol. 119, pp. 13-18.

Vincent, J. H. 1999. Particle Size-Selective Sampling for Particulate Air Contaminants. American Conference of Governmental Industrial Hygienists.

Vitols, V. 1966. Theoretical Limits of Errors Due to Anisokinetic Sampling of Particulate Matter. Journal of Air Pollution Control Association. Vol. 16, No. 2. pp. 79-83.

Watson, H. H. 1954. Errors Due to Anisokinetic Sampling of Aerosols. Industrial Hygiene Quarterly. March, pp. 21-25.

Weiner, R., Okazaki, K., Willeke, K. 1988. Influence of Turbulence on Aerosol Sampling Efficiencies. Atmospheric Environment. Vol. 22 No 5. pp. 917- 928.

White, F. M. 1991. Viscous Fluid Flow. McGraw-Hill, New York.

Witschger, O., Willeke, K., Grinshpun, S. A., Aizenberg, V., Smith, J., Baron, P. 1998. Simplified Method for Testing Personal Inhalable Aerosol Samplers. Journal of Aerosol Science. Vol. 29, No. 7. pp. 855-874. 
Yan, B., Ingham, D. B., Dunnett, S .J. 1994. A Numerical Investigation into the Sampling Performance of a Tube Sampler. Aerosol Science and Technology. Vol. 20, No. 8. pp. 291-302.

Yakhot, V. and Orszag, S. A. 1986. Renormalization Group Analysis of Turbulence. I. Basic Theory. Journal of Scientific Computing. Vol. 1, No. 1, pp. 3-51. 
Appendix A

Results Tables 
Table A.1: Calculated and corrected "filter-only" sampling efficiencies from CFD simulation of the GSP sampler free in the air at a 0-degree orientation to $0.5 \mathrm{~m} / \mathrm{s}$ freestream airflow with corresponding experimental data from (Li, 1999). See Figure 4.16.

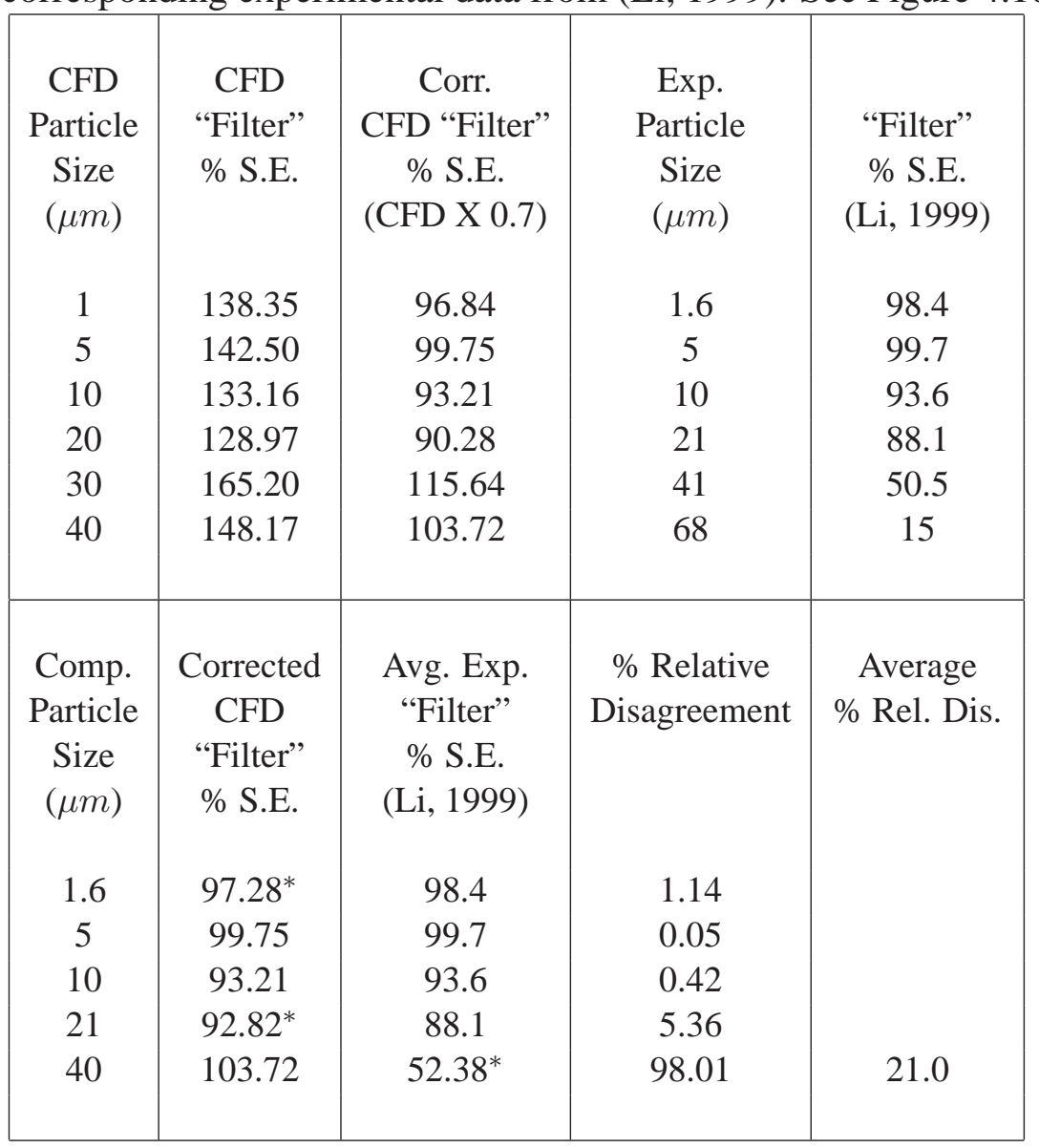

S.E. $=$ Sampling Efficiency; Corr. $=$ Corrected; Exp. = Experiment; Avg. $=$ Average Comp. $=$ Comparison; ${ }^{*}=$ Interpolated;

S.E. $=$ Sampling Efficiency; Rel. $=$ Relative; Dis. $=$ Disagreement 
Table A.2: Calculated and corrected "total" sampling efficiencies from CFD simulation of the GSP sampler free in the air at a 0-degree orientation to $0.5 \mathrm{~m} / \mathrm{s}$ freestream airflow with corresponding experimental data from (Li, 1999). See Figure 4.17.

\begin{tabular}{|c|c|c|c|c|}
\hline $\begin{array}{c}\text { CFD } \\
\text { Particle } \\
\text { Size } \\
(\mu m)\end{array}$ & $\begin{array}{c}\text { CFD } \\
\text { "Total" }\end{array}$ & $\begin{array}{c}\text { Corr. } \\
\text { CFD "Total" } \\
\text { \% S. E. } \\
\text { (CFD X 0.56) }\end{array}$ & $\begin{array}{c}\text { Exp. } \\
\text { Particle } \\
\text { Size } \\
(\mu m)\end{array}$ & $\begin{array}{c}\text { Avg. Exp. } \\
\text { "Total" } \\
\text { \% S. E. } \\
\text { (Li, 1999) }\end{array}$ \\
5 & 166.88 & 93.45 & 1.6 & 98.6 \\
10 & 175.18 & 98.10 & 5 & 100.1 \\
20 & 176.24 & 98.69 & 10 & 96.2 \\
30 & 166.70 & 93.35 & 21 & 89.3 \\
40 & 150.66 & 101.17 & 41 & 74.1 \\
& & 84.01 & 68 & 73 \\
Comp. & Corrected & Avg. Exp. & \% Relative & Average \\
Particle & CFD & "Total" & Disagreement & $\%$ Rel. Dis. \\
Size & "Total" & $\%$ S.E. & & \\
$(\mu m)$ & $\%$ S.E. & (Li, 1999) & & \\
& & & & \\
1.6 & $94.15^{*}$ & 98.6 & 4.51 & \\
5 & 98.1 & 100.1 & 2.00 & \\
10 & 98.69 & 96.2 & 2.59 & \\
21 & $92.45^{*}$ & 89.3 & 3.53 & \\
40 & 84.01 & $75.98 *$ & 10.57 & \\
& & & & \\
\hline
\end{tabular}

S.E. = Sampling Efficiency; Corr. $=$ Corrected; Exp. = Experiment; Avg. $=$ Average Comp. $=$ Comparison $;{ }^{*}=$ Interpolated;

S.E. $=$ Sampling Efficiency; Rel. $=$ Relative; Dis. $=$ Disagreement 
Table A.3: Calculated and corrected "filter-only" sampling efficiencies from CFD simulation of the GSP sampler free in the air at a 90-degree orientation to $0.5 \mathrm{~m} / \mathrm{s}$ freestream airflow with corresponding experimental data from (Li, 1999). See Figure 4.18.

\begin{tabular}{|c|c|c|c|c|}
\hline CFD & CFD & Corr. & Exp. & \\
\hline Particle & "Filter" & CFD "Filter" & Particle & "Filter" \\
\hline $\begin{array}{l}\text { Size } \\
(\mu m)\end{array}$ & $\%$ S.E. & $\begin{array}{c}\text { \% S.E. } \\
\text { (CFD X 1.92) }\end{array}$ & $\begin{array}{l}\text { Size } \\
(\mu m)\end{array}$ & $\begin{array}{c}\text { \% S.E. } \\
(\mathrm{Li}, 1999)\end{array}$ \\
\hline 1 & 59.160 & 113.59 & - & - \\
\hline 5 & 51.22 & 98.35 & 5 & 84.3 \\
\hline 10 & 36.34 & 69.77 & 10 & 75.3 \\
\hline 20 & 21.53 & 41.33 & 21 & 44 \\
\hline 30 & 6.01 & 11.54 & 41 & 4 \\
\hline 40 & 0.75 & 1.44 & 68 & 4.8 \\
\hline Comp. & Corrected & Avg. Exp. & $\%$ Relative & Average \\
\hline Particle & CFD & "Filter" & Disagreement & \% Rel. Dis. \\
\hline $\begin{array}{l}\text { Size } \\
(\mu m)\end{array}$ & $\begin{array}{l}\text { "Filter" } \\
\% \text { S.E. }\end{array}$ & $\begin{array}{c}\% \text { S.E. } \\
(\mathrm{Li}, 1999)\end{array}$ & & \\
\hline 5 & 98.35 & 84.3 & 16.66 & \\
\hline 10 & 69.77 & 75.3 & 7.34 & \\
\hline 21 & $38.4^{*}$ & 44 & 12.73 & \\
\hline 40 & 1.44 & $6^{*}$ & 76.06 & 28.2 \\
\hline
\end{tabular}

S.E. $=$ Sampling Efficiency; Corr. $=$ Corrected; Exp. = Experiment; Avg. $=$ Average Comp. $=$ Comparison $;{ }^{*}=$ Interpolated;

S.E. $=$ Sampling Efficiency; Rel. $=$ Relative; Dis. $=$ Disagreement 
Table A.4: Calculated and corrected "total" sampling efficiencies from CFD simulation of the GSP sampler free in the air at a 90-degree orientation to $0.5 \mathrm{~m} / \mathrm{s}$ freestream airflow with corresponding experimental data from (Li, 1999). See Figure 4.19.

\begin{tabular}{|c|c|c|c|c|}
\hline $\begin{array}{c}\text { CFD } \\
\text { Particle } \\
\text { Size } \\
(\mu m)\end{array}$ & $\begin{array}{c}\text { CFD } \\
\text { "Total" } \\
\text { \% S.E. }\end{array}$ & $\begin{array}{c}\text { Corr. } \\
\text { CFD "Total" } \\
\text { \% S.E. } \\
\text { (CFD X 1.76) }\end{array}$ & $\begin{array}{c}\text { Exp. } \\
\text { Particle } \\
\text { Size } \\
(\mu m)\end{array}$ & $\begin{array}{c}\text { Avg. Exp. } \\
\text { "Total" } \\
\text { \% S.E. } \\
\text { (Li, 1999) }\end{array}$ \\
1 & 70.256 & 123.65 & - & - \\
5 & 65.03 & 114.46 & 5 & 85.2 \\
10 & 51.28 & 90.25 & 10 & 81.8 \\
20 & 24.05 & 42.33 & 21 & 57.4 \\
30 & 6.30 & 11.09 & 41 & 11.9 \\
40 & 0.75 & 1.32 & 68 & 5.8 \\
& & & & \\
\hline & & & & \\
Comp. & Corrected & Avg. Exp. & Relative & Average \\
Particle & CFD & "Total" & Disagreement & $\%$ Rel. Dis. \\
Size & "Total" & $\%$ S.E. & & \\
$(\mu m)$ & $\%$ S.E. & (Li, 1999) & & \\
& & & & \\
5 & 114.46 & 85.2 & 34.34 & \\
10 & 90.25 & 81.8 & 10.32 & \\
21 & $39.2^{*}$ & 57.4 & 31.71 & \\
40 & 1.32 & $14.175^{*}$ & 90.71 & \\
& & & & \\
\hline
\end{tabular}

S.E. $=$ Sampling Efficiency; Corr. $=$ Corrected; Exp. $=$ Experiment; Avg. $=$ Average Comp. $=$ Comparison $;{ }^{*}=$ Interpolated;

S.E. $=$ Sampling Efficiency; Rel. $=$ Relative; Dis. $=$ Disagreement 
Table A.5: Calculated and corrected "total" sampling efficiencies from CFD simulation of the GSP sampler free in the air at a 180-degree orientation to $0.5 \mathrm{~m} / \mathrm{s}$ freestream airflow with corresponding experimental data from (Li, 1999). See Figure 4.20.

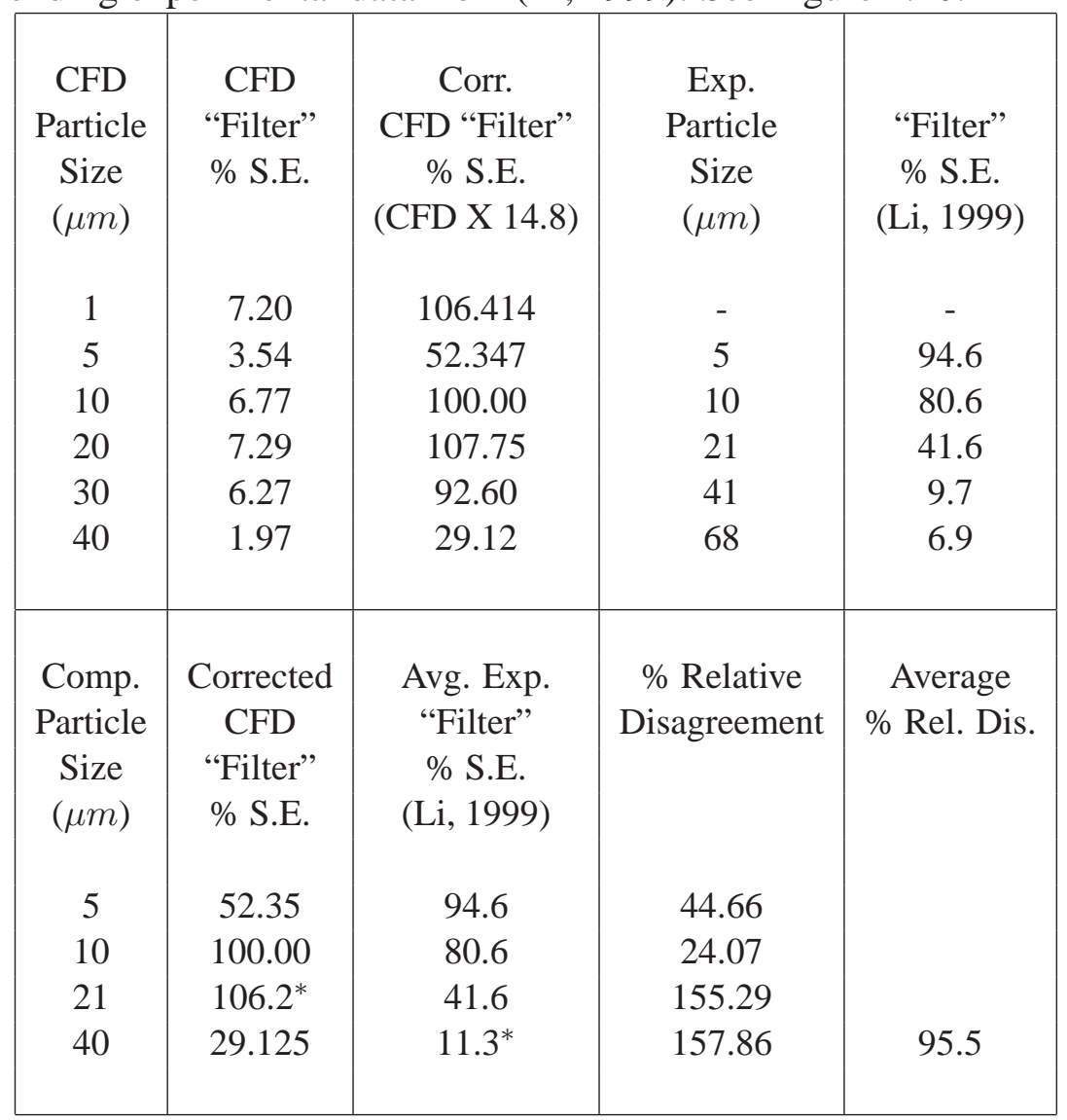

S.E. $=$ Sampling Efficiency; Corr. $=$ Corrected; Exp. $=$ Experiment; Avg. $=$ Average Comp. $=$ Comparison $;{ }^{*}=$ Interpolated;

S.E. $=$ Sampling Efficiency; Rel. $=$ Relative; Dis. $=$ Disagreement 
Table A.6: Calculated and corrected "total" sampling efficiencies from CFD simulation of the GSP sampler free in the air at a 180-degree orientation to $0.5 \mathrm{~m} / \mathrm{s}$ freestream airflow with corresponding experimental data from (Li, 1999). See Figure 4.21.

\begin{tabular}{|c|c|c|c|c|}
\hline $\begin{array}{l}\text { CFD } \\
\text { Particle } \\
\text { Size } \\
(\mu m)\end{array}$ & $\begin{array}{l}\text { CFD } \\
\text { "Total" } \\
\% \text { S.E. }\end{array}$ & $\begin{array}{c}\text { Corr. } \\
\text { CFD “Total” } \\
\% \text { S.E. } \\
\text { (CFD X 6.9) }\end{array}$ & $\begin{array}{l}\text { Exp. } \\
\text { Particle } \\
\text { Size } \\
(\mu m)\end{array}$ & $\begin{array}{c}\text { Avg. Exp. } \\
\text { "Total" } \\
\text { \% S.E. } \\
\text { (Li, 1999) }\end{array}$ \\
\hline $\begin{array}{c}1 \\
5 \\
10 \\
20 \\
30 \\
40\end{array}$ & $\begin{array}{c}11.33 \\
11.34 \\
11.26 \\
10.05 \\
7.29 \\
1.97\end{array}$ & $\begin{array}{c}78.165 \\
78.221 \\
77.68 \\
69.35 \\
50.31 \\
13.60\end{array}$ & $\begin{array}{c}- \\
5 \\
10 \\
21 \\
41 \\
68\end{array}$ & $\begin{array}{c}- \\
95.2 \\
84.9 \\
47.9 \\
16.6 \\
8.9\end{array}$ \\
\hline $\begin{array}{c}\text { Comp. } \\
\text { Particle } \\
\text { Size } \\
(\mu m)\end{array}$ & $\begin{array}{c}\text { Corrected } \\
\text { CFD } \\
\text { "Total" } \\
\% \text { S.E. }\end{array}$ & $\begin{array}{l}\text { Avg. Exp. } \\
\text { "Total" } \\
\text { \% S.E. } \\
\text { (Li, 1999) }\end{array}$ & $\begin{array}{c}\% \text { Relative } \\
\text { Disagreement }\end{array}$ & $\begin{array}{c}\text { Average } \\
\% \text { Rel. Dis. }\end{array}$ \\
\hline $\begin{array}{c}5 \\
10 \\
21 \\
40\end{array}$ & $\begin{array}{c}78.221 \\
77.679 \\
67.4^{*} \\
13.597\end{array}$ & $\begin{array}{c}95.2 \\
84.9 \\
47.9 \\
18.17^{*}\end{array}$ & $\begin{array}{c}17.84 \\
8.51 \\
40.71 \\
25.15\end{array}$ & 23.05 \\
\hline
\end{tabular}

S.E. $=$ Sampling Efficiency; Corr. $=$ Corrected; Exp. = Experiment; Avg. $=$ Average Comp. $=$ Comparison ${ }^{*}=$ Interpolated;

S.E. $=$ Sampling Efficiency; Rel. = Relative; Dis. $=$ Disagreement 
Table A.7: Calculated and corrected "filter-only" sampling efficiencies from CFD simulation of the GSP sampler free in the air at a 0-degree orientation to $1.0 \mathrm{~m} / \mathrm{s}$ freestream airflow with corresponding experimental data from (Li, 1999). See Figure 4.22.

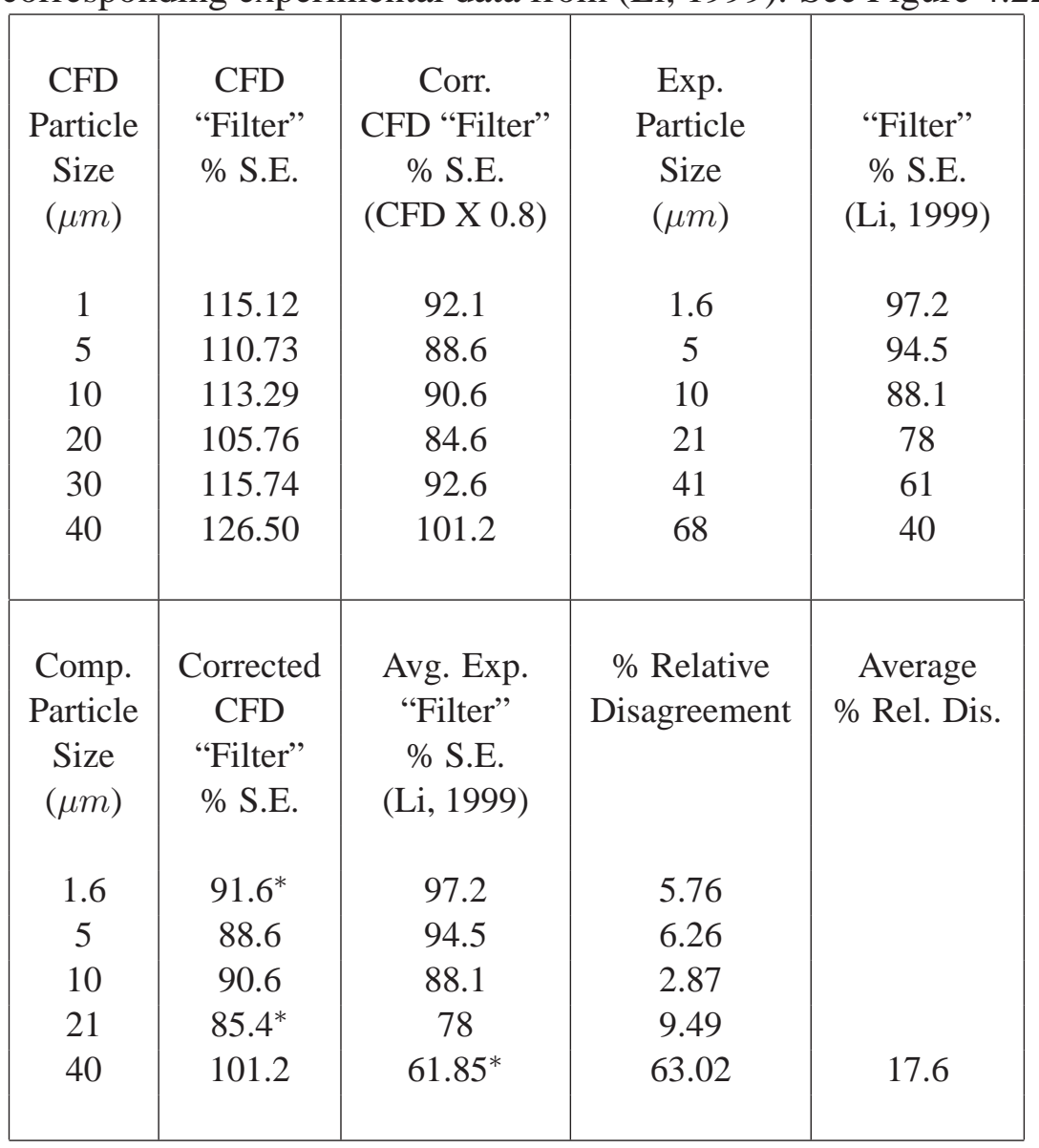

S.E. $=$ Sampling Efficiency; Corr. $=$ Corrected; Exp. = Experiment; Avg. $=$ Average Comp. $=$ Comparison; ${ }^{*}=$ Interpolated;

S.E. = Sampling Efficiency; Rel. = Relative; Dis. = Disagreement 
Table A.8: Calculated and corrected "total" sampling efficiencies from CFD simulation of the GSP sampler free in the air at a 0-degree orientation to $1.0 \mathrm{~m} / \mathrm{s}$ freestream airflow with corresponding experimental data from (Li, 1999). See Figure 4.23.

\begin{tabular}{|c|c|c|c|c|}
\hline $\begin{array}{c}\text { CFD } \\
\text { Particle } \\
\text { Size } \\
(\mu m)\end{array}$ & $\begin{array}{c}\text { CFD } \\
\text { "Total" }\end{array}$ & $\begin{array}{c}\text { Corr. } \\
\text { CFD "Total" } \\
\text { \% S.E. } \\
\text { (CFD X 0.71) }\end{array}$ & $\begin{array}{c}\text { Exp. } \\
\text { Particle } \\
\text { Size } \\
(\mu m)\end{array}$ & $\begin{array}{c}\text { Avg. Exp. } \\
\text { "Total" } \\
\% \text { S.E. } \\
\text { (Li, 1999) }\end{array}$ \\
5 & 136.42 & 96.9 & 1.6 & 97.2 \\
10 & 132.28 & 93.9 & 5 & 95.3 \\
20 & 145.10 & 103.0 & 10 & 95.4 \\
30 & 137.56 & 97.7 & 21 & 94.6 \\
40 & 134.35 & 95.2 & 41 & 103 \\
& & 95.4 & 68 & 101.3 \\
Comp. & Corrected & Avg. Exp. & \% Relative & Average \\
Particle & CFD & "Total" & Disagreement & $\%$ Rel. Dis. \\
Size & "Total" & $\%$ S.E. & & \\
$(\mu m)$ & $\%$ S.E. & (Li, 1999) & & \\
& & & & \\
1.6 & $96.4^{*}$ & 97.2 & 0.82 & \\
5 & 93.9 & 95.3 & 1.45 & \\
10 & 103.0 & 95.4 & 7.99 & \\
21 & $97.4^{*}$ & 94.6 & 2.96 & \\
40 & 95.4 & $102.58^{*}$ & 7.01 & \\
& & & & \\
\hline
\end{tabular}

S.E. = Sampling Efficiency; Corr. $=$ Corrected; Exp. = Experiment; Avg. $=$ Average Comp. $=$ Comparison $;{ }^{*}=$ Interpolated;

S.E. $=$ Sampling Efficiency; Rel. $=$ Relative; Dis. $=$ Disagreement 
Table A.9: Calculated and corrected "filter" sampling efficiencies from CFD simulation of the GSP sampler free in the air at a 90-degree orientation to $1.0 \mathrm{~m} / \mathrm{s}$ freestream airflow with corresponding experimental data from ( $\mathrm{Li}, 1999)$. See Figure 4.24.

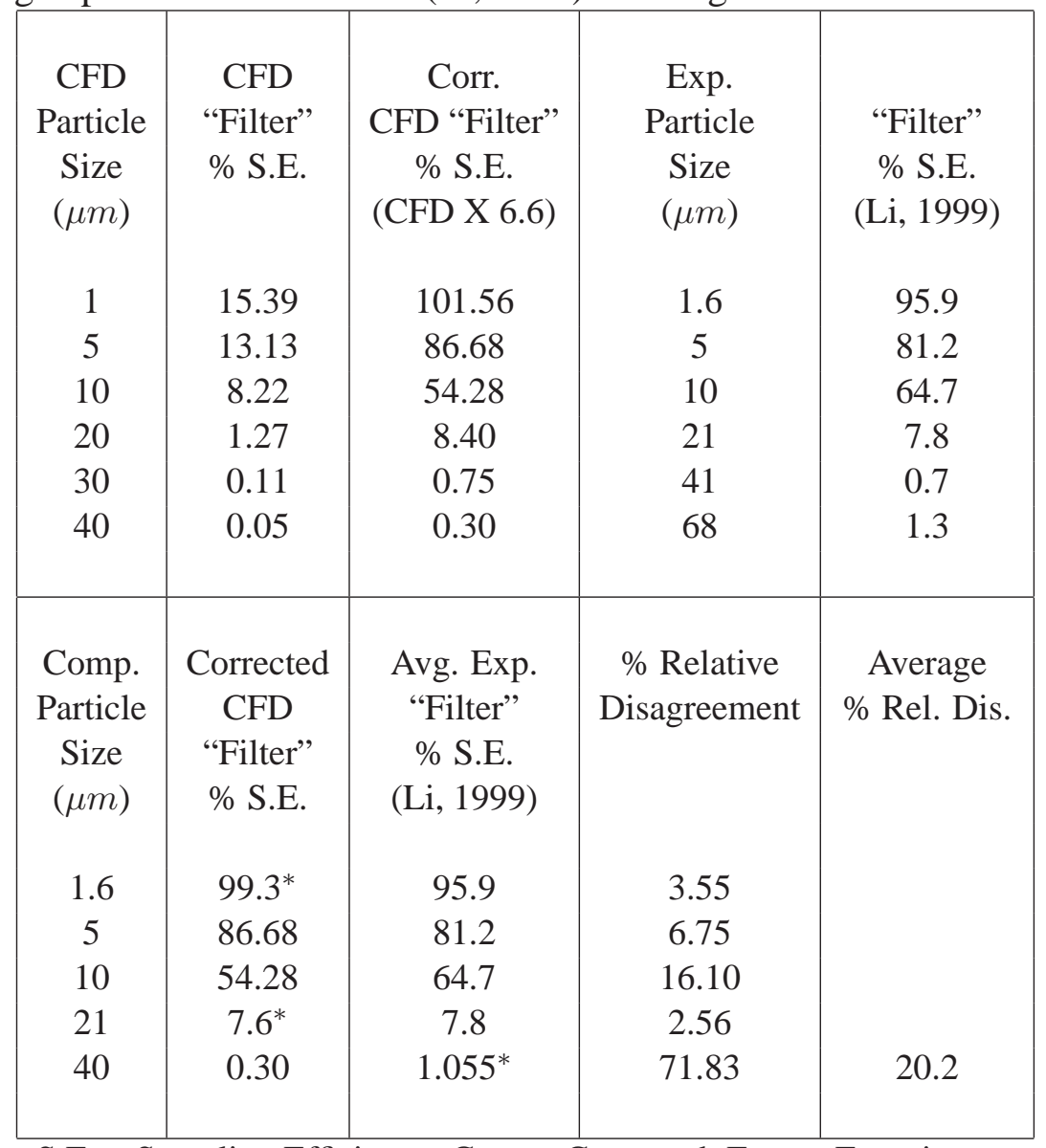

S.E. $=$ Sampling Efficiency; Corr. $=$ Corrected; Exp. $=$ Experiment; Avg. $=$ Average Comp. $=$ Comparison; ${ }^{*}=$ Interpolated;

S.E. $=$ Sampling Efficiency; Rel. $=$ Relative; Dis. $=$ Disagreement 
Table A.10: Calculated and corrected "total" sampling efficiencies from CFD simulation of the GSP sampler free in the air at a 90-degree orientation to $1.0 \mathrm{~m} / \mathrm{s}$ freestream airflow with corresponding experimental data from (Li, 1999). See Figure 4.25.

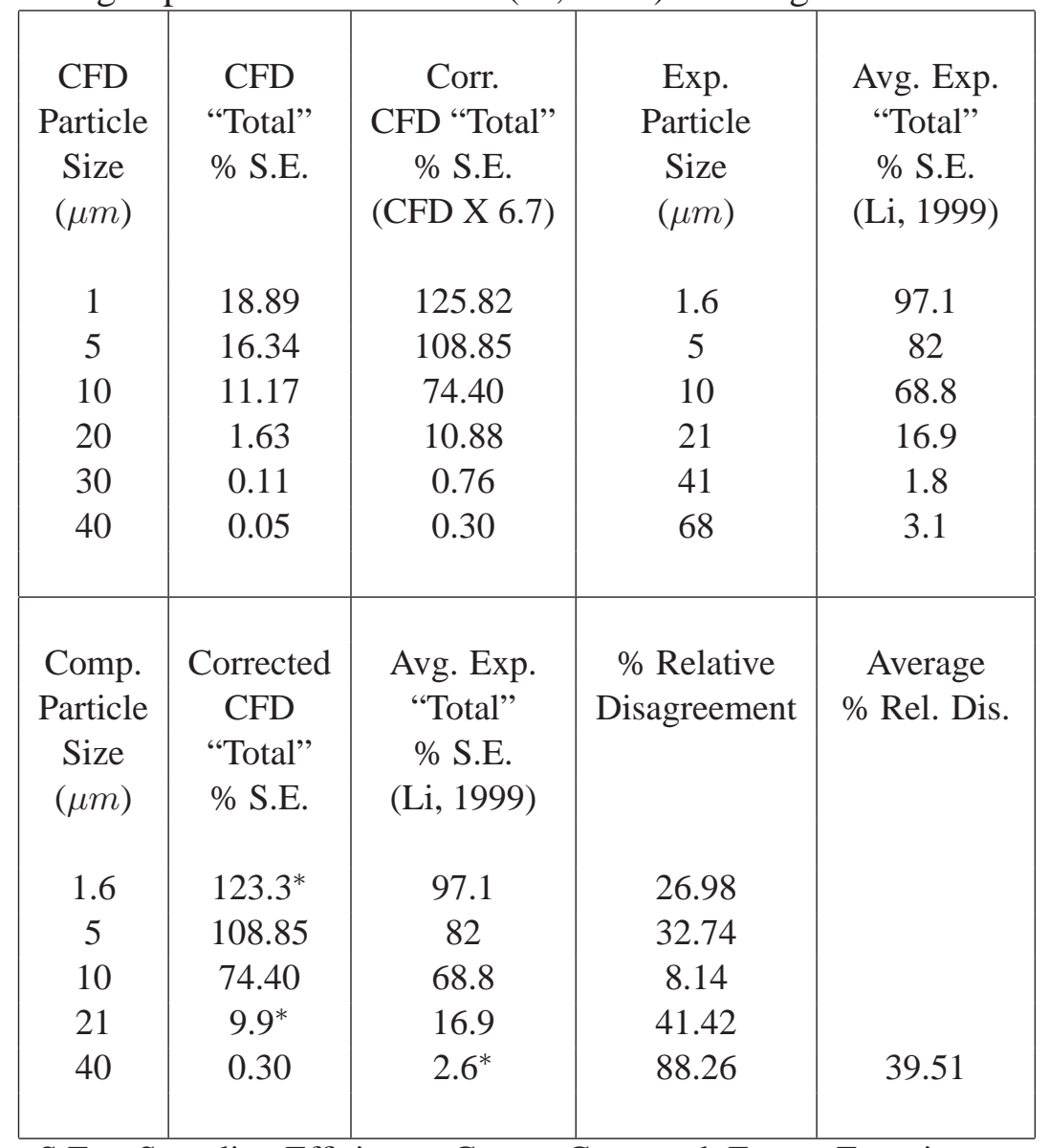

S.E. $=$ Sampling Efficiency; Corr. $=$ Corrected; Exp. $=$ Experiment; Avg. $=$ Average Comp. $=$ Comparison ${ }^{*}=$ Interpolated;

S.E. = Sampling Efficiency; Rel. = Relative; Dis. = Disagreement 
Table A.11: Calculated and corrected "filter" sampling efficiencies from CFD simulation of the GSP sampler free in the air at a 180-degree orientation to $1.0 \mathrm{~m} / \mathrm{s}$ freestream airflow with corresponding experimental data from (Li, 1999). See Figure 4.26.

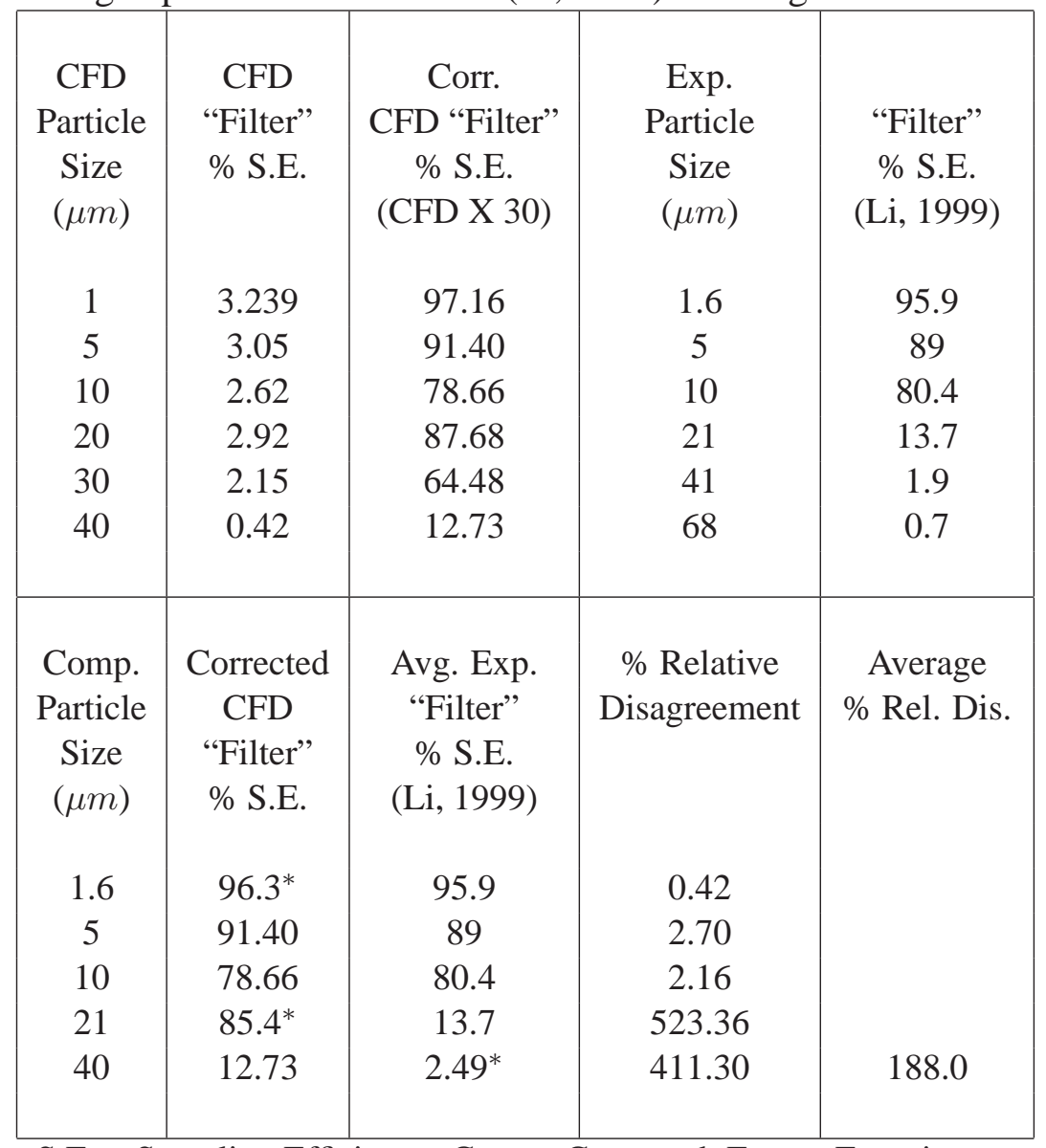

S.E. $=$ Sampling Efficiency; Corr. $=$ Corrected; Exp. $=$ Experiment; Avg. $=$ Average Comp. $=$ Comparison; ${ }^{*}=$ Interpolated;

S.E. $=$ Sampling Efficiency; Rel. $=$ Relative; Dis. $=$ Disagreement 
Table A.12: Calculated and corrected "total" sampling efficiencies from CFD simulation of the GSP sampler free in the air at a 180-degree orientation to $1.0 \mathrm{~m} / \mathrm{s}$ freestream airflow with corresponding experimental data from (Li, 1999). See Figure 4.27.

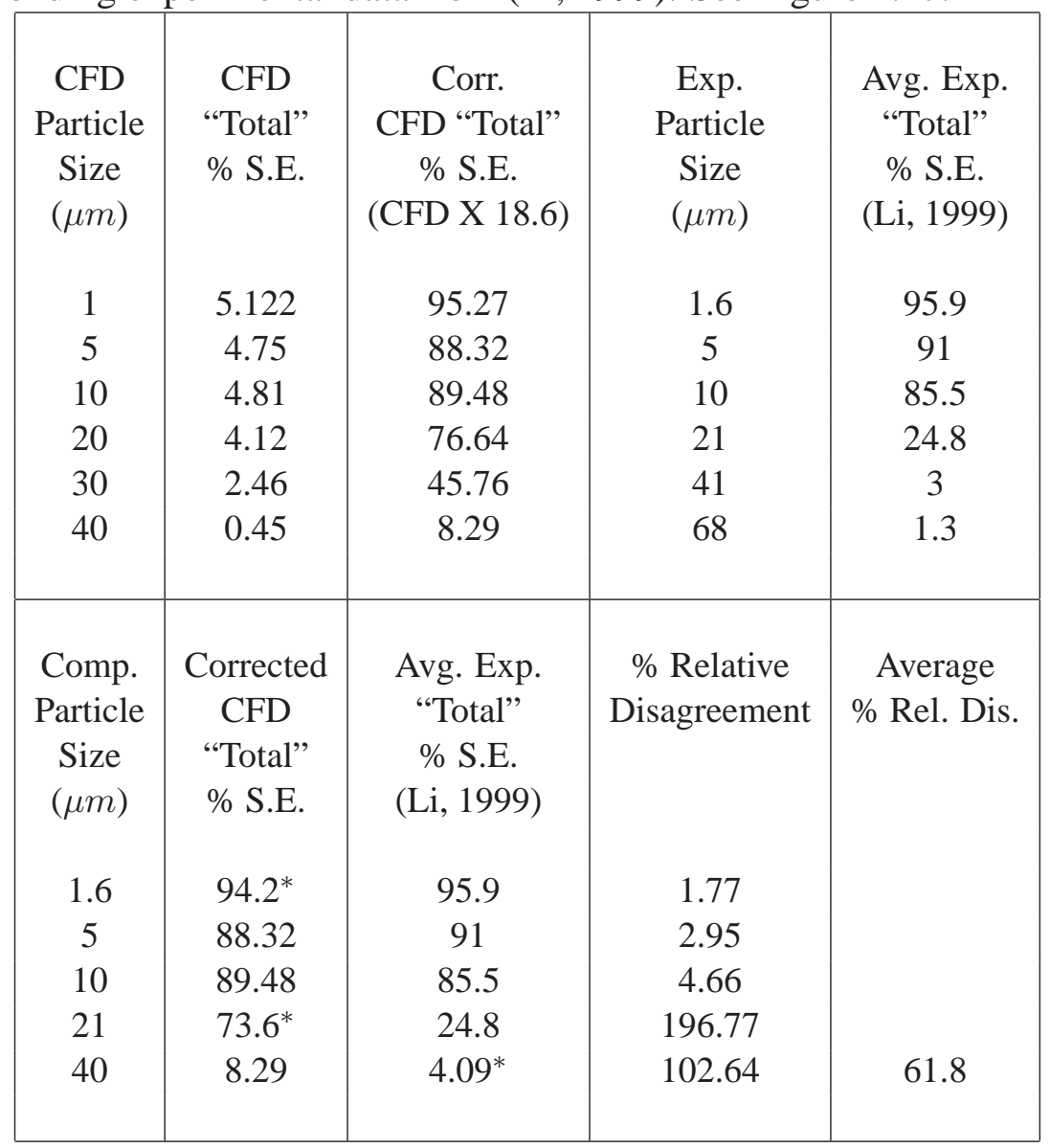

S.E. = Sampling Efficiency; Corr. $=$ Corrected; Exp. = Experiment; Avg. $=$ Average Comp. $=$ Comparison $;{ }^{*}=$ Interpolated;

S.E. $=$ Sampling Efficiency; Rel. $=$ Relative; Dis. $=$ Disagreement 
Table A.13: Calculated and corrected " $3 \mathrm{X}$ averaging" sampling efficiencies from CFD simulation of the GSP sampler mounted on a box-shaped bluff body in $0.5 \mathrm{~m} / \mathrm{s}$ freestream airflow with corresponding experimental data from (Kenny et al. 1997). See Figure 4.28.

\begin{tabular}{|c|c|c|c|c|}
\hline $\begin{array}{l}\text { CFD } \\
\text { Particle } \\
\text { Size } \\
(\mu m)\end{array}$ & $\begin{array}{c}\text { CFD } \\
\text { "3X" } \\
\% \text { S.E. }\end{array}$ & $\begin{array}{c}\text { Corr. } \\
\text { CFD “3X” } \\
\% \text { S.E. } \\
\text { (CFD X 1.5) }\end{array}$ & $\begin{array}{l}\text { Exp. } \\
\text { Particle } \\
\text { Size } \\
(\mu m)\end{array}$ & $\begin{array}{c}\text { Directional } \\
\text { Average } \\
\% \text { S.E. } \\
\text { (Kenny et al., 1997) }\end{array}$ \\
\hline $\begin{array}{c}1 \\
6 \\
10 \\
16 \\
20 \\
50\end{array}$ & $\begin{array}{l}65.48 \\
40.85 \\
62.78 \\
76.36 \\
60.10 \\
15.59\end{array}$ & $\begin{array}{c}98.22 \\
61.27 \\
94.17 \\
114.54 \\
90.15 \\
23.39\end{array}$ & $\begin{array}{c}6 \\
19.4 \\
21.2 \\
30.7 \\
41.8 \\
55.2 \\
85 \\
96\end{array}$ & $\begin{array}{l}93.2 \\
65.5 \\
69.3 \\
59.0 \\
38.5 \\
24.5 \\
37.8 \\
23.2\end{array}$ \\
\hline $\begin{array}{l}\text { Comp. } \\
\text { Particle } \\
\text { Size } \\
(\mu m)\end{array}$ & $\begin{array}{c}\text { Corrected } \\
\text { CFD } \\
\text { "3X" } \\
\% \text { S.E. }\end{array}$ & $\begin{array}{c}\text { Avg. Exp. } \\
\text { Directional Avg. } \\
\% \text { S.E. } \\
\text { (Kenny et al., 1997) }\end{array}$ & $\begin{array}{c}\% \text { Relative } \\
\text { Disagreement }\end{array}$ & $\begin{array}{c}\text { Average } \\
\% \text { Rel. Dis. }\end{array}$ \\
\hline $\begin{array}{c}6 \\
19.4 \\
21.2 \\
30.7 \\
41.8\end{array}$ & $\begin{array}{c}61.27 \\
93.81^{*} \\
89.17^{*} \\
81.45^{*} \\
72.42^{*}\end{array}$ & $\begin{array}{l}93.2 \\
65.5 \\
69.3 \\
59.0 \\
38.5\end{array}$ & $\begin{array}{l}34.24 \\
43.22 \\
28.62 \\
38.05 \\
88.10\end{array}$ & 46.5 \\
\hline
\end{tabular}

S.E. $=$ Sampling Efficiency; Corr. $=$ Corrected; Exp. $=$ Experiment;

Avg. = Average; Comp. = Comparison; ${ }^{*}=$ Interpolated;

S.E. = Sampling Efficiency; Rel. = Relative; Dis. = Disagreement

"3X" = Directional Averaging by Eqn. 4.2 
Table A.14: Calculated and corrected "Witschger averaging" sampling efficiencies from CFD simulation of the GSP sampler mounted on a box-shaped bluff body in $0.5 \mathrm{~m} / \mathrm{s}$ freestream airflow with corresponding experimental data from (Kenny et al. 1997). See Figure 4.29.

\begin{tabular}{|c|c|c|c|c|}
\hline $\begin{array}{l}\text { CFD } \\
\text { Particle } \\
\text { Size } \\
(\mu m)\end{array}$ & $\begin{array}{c}\text { CFD } \\
\text { "Witschger" } \\
\text { \% S.E. }\end{array}$ & $\begin{array}{c}\text { Corr. } \\
\text { CFD "Witschger" } \\
\% \text { S.E. } \\
\text { (CFD X 1.5) }\end{array}$ & $\begin{array}{l}\text { Exp. } \\
\text { Particle } \\
\text { Size } \\
(\mu m)\end{array}$ & $\begin{array}{c}\text { Directional } \\
\text { Average } \\
\% \text { S.E. } \\
\text { (Kenny et al., 1997) }\end{array}$ \\
\hline $\begin{array}{c}1 \\
6 \\
10 \\
16 \\
20 \\
50\end{array}$ & $\begin{array}{l}49.18 \\
30.68 \\
47.18 \\
57.33 \\
45.07 \\
11.69\end{array}$ & $\begin{array}{l}73.764 \\
46.014 \\
70.775 \\
85.990 \\
67.610 \\
17.542\end{array}$ & $\begin{array}{c}6 \\
19.4 \\
21.2 \\
30.7 \\
41.8 \\
55.2 \\
85 \\
96\end{array}$ & $\begin{array}{l}93.167 \\
65.500 \\
69.333 \\
59.000 \\
38.500 \\
24.500 \\
37.833 \\
23.167\end{array}$ \\
\hline $\begin{array}{l}\text { Comp. } \\
\text { Particle } \\
\text { Size } \\
(\mu m)\end{array}$ & $\begin{array}{l}\text { Corrected } \\
\text { CFD } \\
\text { "Witschger" } \\
\text { \% S.E. }\end{array}$ & $\begin{array}{c}\text { Avg. Exp. } \\
\text { Directional Avg. } \\
\text { \% S.E. } \\
\text { (Kenny et al., 1997) }\end{array}$ & $\begin{array}{c}\% \text { Relative } \\
\text { Disagreement }\end{array}$ & $\begin{array}{c}\text { Average } \\
\% \text { Rel. Dis. }\end{array}$ \\
\hline $\begin{array}{c}6 \\
19.4 \\
21.2 \\
30.7 \\
41.8\end{array}$ & $\begin{array}{l}46.020 \\
70.360 \\
65.600 \\
49.750 \\
31.220\end{array}$ & $\begin{array}{l}93.167 \\
65.500 \\
69.333 \\
59.000 \\
38.500\end{array}$ & $\begin{array}{c}102.45 \\
6.91 \\
5.69 \\
18.59 \\
23.32\end{array}$ & 31.39 \\
\hline
\end{tabular}

S.E. $=$ Sampling Efficiency; Corr. $=$ Corrected; Exp. $=$ Experiment;

Avg. $=$ Average $;$ Comp. $=$ Comparison ${ }^{*}=$ Interpolated;

S.E. $=$ Sampling Efficiency; Rel. $=$ Relative; Dis. $=$ Disagreement

"Witschger" = Directional Averaging by Eqn. 4.1 
Table A.15: Sampling efficiency values from (Kenny et al. 1997) for the GSP sampler mounted on a step-wise rotating mannequin in a wind tunnel. See Figure 4.30.

\begin{tabular}{|c|c|c|c|}
\hline $\begin{array}{c}\text { Wind } \\
\text { Speed } \\
(\mathrm{m} / \mathrm{s})\end{array}$ & $\begin{array}{c}\text { Particle } \\
\text { Size } \\
(\mu \mathrm{m})\end{array}$ & $\begin{array}{c}\text { Avg. } \\
\text { Samp. Effic. } \\
(\%)\end{array}$ & $\begin{array}{c}\text { Standard } \\
\text { Deviation }\end{array}$ \\
& & & \\
1 & 7.5 & 84.83 & 3.66 \\
1 & 19.4 & 66.67 & 2.34 \\
1 & 21.2 & 54.83 & 2.79 \\
1 & 23.3 & 38.33 & 2.73 \\
1 & 30.7 & 40.00 & 0.89 \\
1 & 41.8 & 22.33 & 1.37 \\
1 & 55.2 & 29.33 & 2.94 \\
1 & 81 & 27.67 & 2.42 \\
1 & 96 & 21.67 & 2.34 \\
& & & \\
4 & 6 & 46.83 & 6.05 \\
4 & 19.4 & 12.60 & 6.50 \\
4 & 21.2 & 14.25 & 0.96 \\
4 & 23.3 & 10.60 & 5.50 \\
4 & 30.7 & 12.00 & 4.08 \\
4 & 41.8 & 8.80 & 1.92 \\
4 & 55.2 & 10.50 & 3.42 \\
4 & 81 & 19.83 & 2.93 \\
4 & 96 & 27.00 & 5.20 \\
& & & \\
\hline
\end{tabular}


Table A.16: Calculated and corrected " $3 \mathrm{X}$ " and "Witschger" averaged sampling efficiency values from CFD simulation of the GSP sampler mounted on a box-shaped bluff body in 1.25 and $2.0 \mathrm{~m} / \mathrm{s}$ freestream airflow. See Figure 4.30.

\begin{tabular}{|c|c|c|c|c|c|}
\hline $\begin{array}{c}\text { Wind } \\
\text { Speed } \\
(\mathrm{m} / \mathrm{s})\end{array}$ & $\begin{array}{c}\text { Particle } \\
\text { Size } \\
(\mu m)\end{array}$ & $\begin{array}{c}\text { 3X Avg. } \\
\text { Samp. Effic. } \\
(\%)\end{array}$ & $\begin{array}{c}\text { Corrected } \\
\text { 3X Avg. } \\
(\%)\end{array}$ & $\begin{array}{c}\text { Wits. Avg. } \\
\text { Samp. Effic } \\
(\%)\end{array}$ & $\begin{array}{c}\text { Corr. (2.5) } \\
\text { Wits. Avg. } \\
(\%)\end{array}$ \\
& & & & & \\
1.25 & 1 & 60.78 & 91.17 & 45.61 & 114.03 \\
1.25 & 6 & 23.71 & 35.56 & 17.81 & 44.52 \\
1.25 & 10 & 23.45 & 35.17 & 17.61 & 44.03 \\
1.25 & 16 & 21.01 & 31.52 & 15.78 & 39.46 \\
1.25 & 20 & 11.73 & 17.60 & 8.80 & 22.0 \\
1.25 & 50 & 19.12 & 28.67 & 14.34 & 35.8 \\
& & & & & \\
& & & Corr. (1.9) & & \\
2.0 & 1 & 51.99 & 98.79 & 39.00 & 97.49 \\
2.0 & 6 & 12.27 & 23.31 & 9.20 & 23.0 \\
2.0 & 10 & 16.73 & 31.78 & 12.55 & 31.36 \\
2.0 & 16 & 11.11 & 21.11 & 8.33 & 20.83 \\
2.0 & 20 & 11.14 & 21.16 & 8.35 & 20.89 \\
2.0 & 50 & 10.44 & 19.83 & 7.83 & 19.57 \\
& & & & & \\
\hline
\end{tabular}

Samp. Effic. $=$ Sampling Efficiency; Corr. $=$ Corrected

Wits. = Witschger; Avg. = Average

"Witschger" = Directional Averaging by Eqn. 4.1

" $3 \mathrm{X} "=$ Directional Averaging by Eqn. 4.2 
Table A.17: Calculated and corrected "total" sampling efficiencies from CFD simulation of the IOM sampler free in the air at a 0 -degree orientation to $0.5 \mathrm{~m} / \mathrm{s}$ freestream airflow with corresponding experimental data from (Li, 1999). See Figure 4.31.

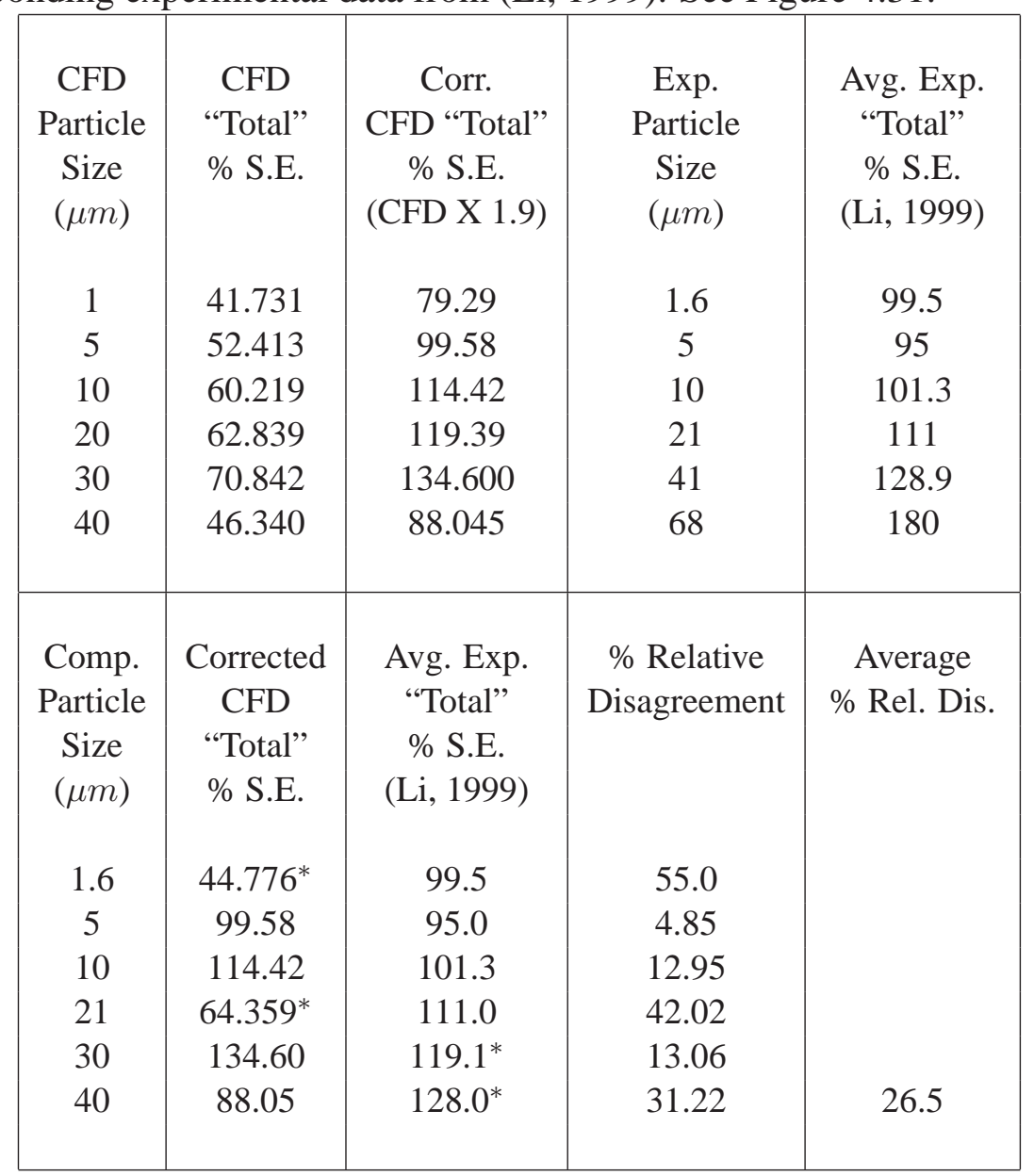

S.E. $=$ Sampling Efficiency; Corr. $=$ Corrected; Exp. = Experiment; Avg. $=$ Average Comp. $=$ Comparison; ${ }^{*}=$ Interpolated;

S.E. = Sampling Efficiency; Rel. = Relative; Dis. $=$ Disagreement 
Table A.18: Calculated and corrected "total" sampling efficiencies from CFD simulation of the IOM sampler free in the air at a 90-degree orientation to $0.5 \mathrm{~m} / \mathrm{s}$ freestream airflow with corresponding experimental data from (Li, 1999). See Figure 4.32.

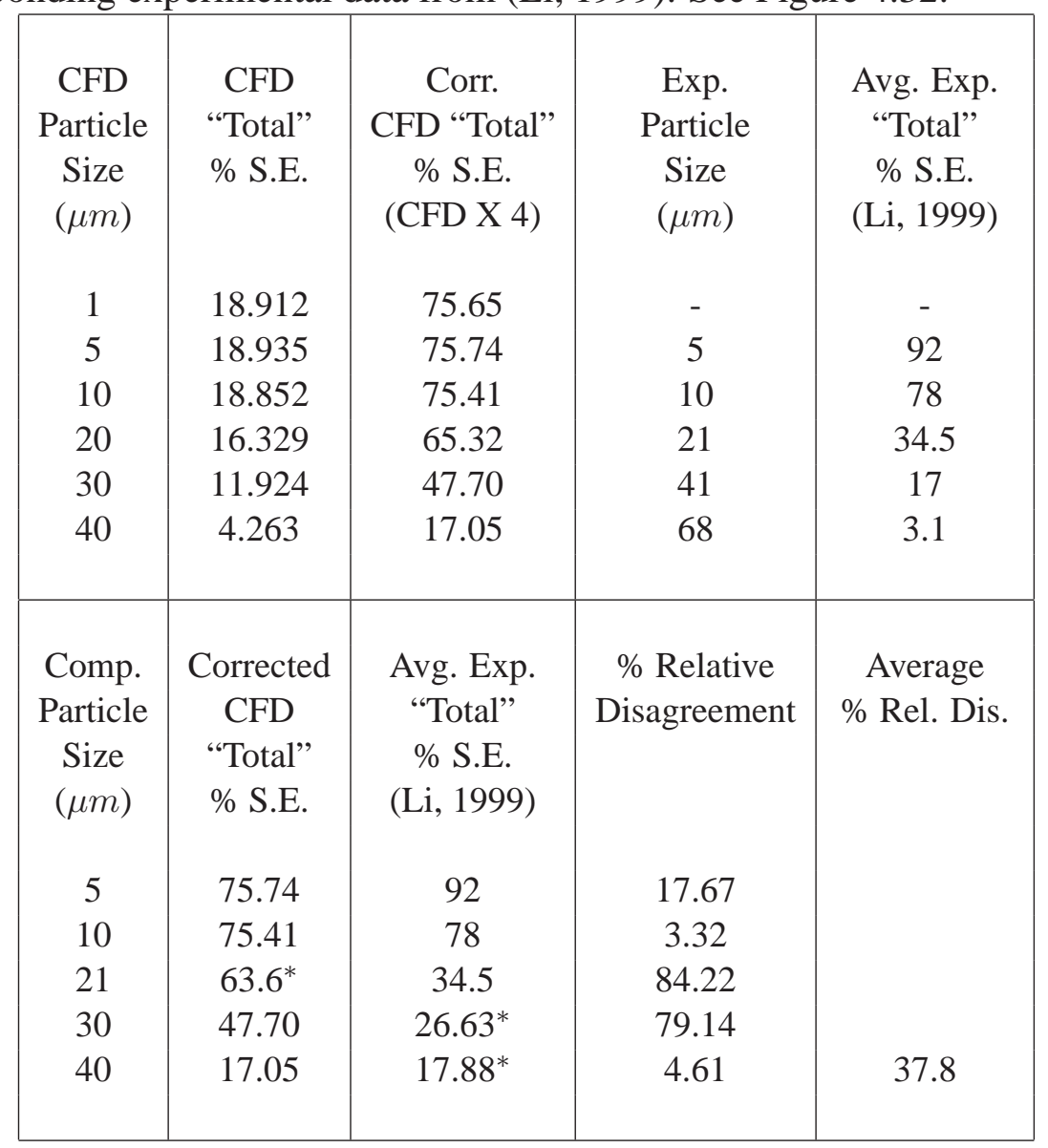

S.E. $=$ Sampling Efficiency; Corr. $=$ Corrected; Exp. $=$ Experiment; Avg. $=$ Average Comp. $=$ Comparison ${ }^{*}=$ Interpolated;

S.E. $=$ Sampling Efficiency; Rel. $=$ Relative; Dis. $=$ Disagreement 
Table A.19: Calculated and corrected "total" sampling efficiencies from CFD simulation of the IOM sampler free in the air at a 180-degree orientation to $0.5 \mathrm{~m} / \mathrm{s}$ freestream airflow with corresponding experimental data from (Li, 1999). See Figure 4.33.

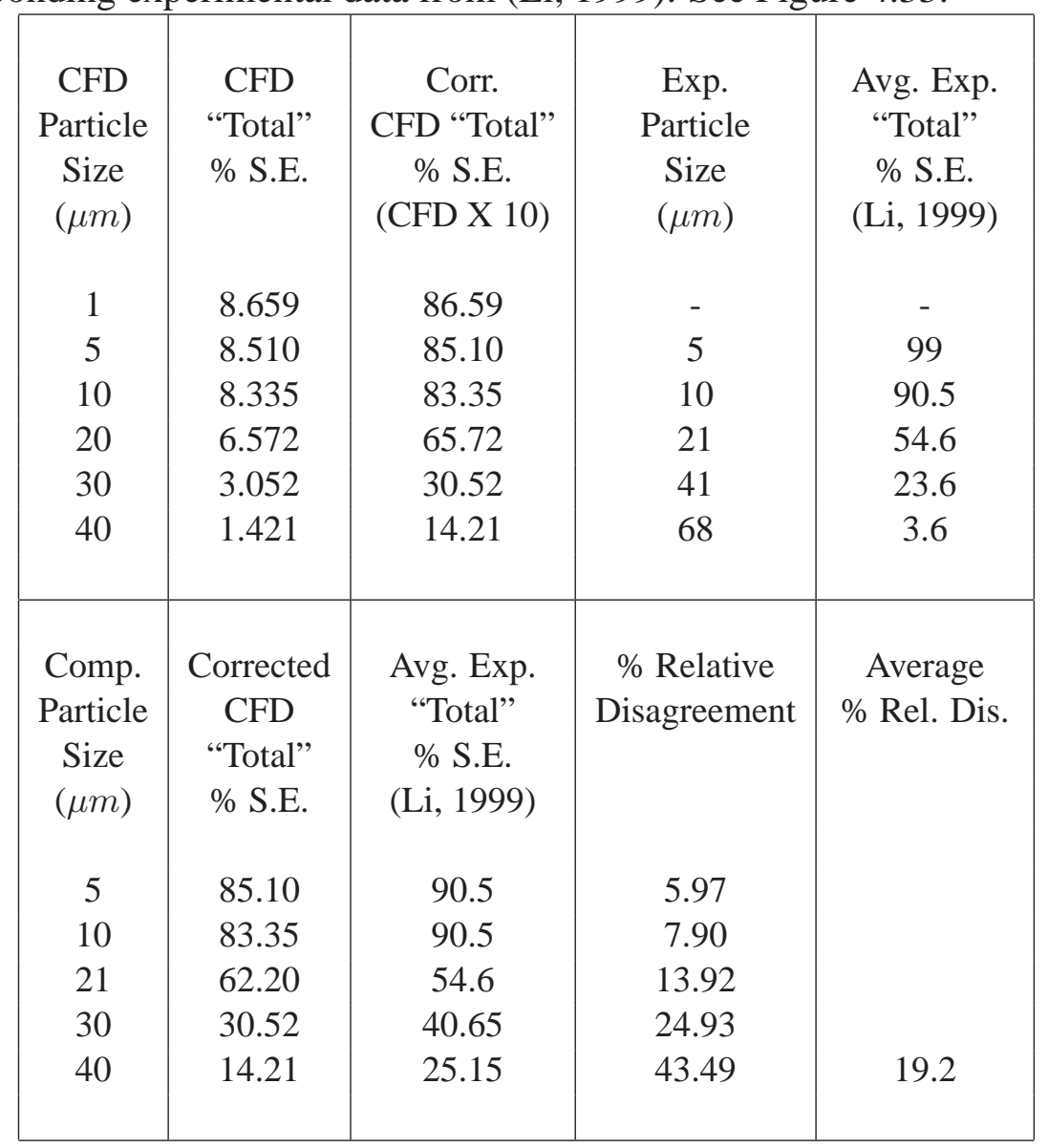

S.E. $=$ Sampling Efficiency; Corr. $=$ Corrected; Exp. $=$ Experiment; Avg. $=$ Average Comp. $=$ Comparison; ${ }^{*}=$ Interpolated;

S.E. $=$ Sampling Efficiency; Rel. $=$ Relative; Dis. $=$ Disagreement 
Table A.20: Calculated and corrected "total" sampling efficiencies from CFD simulation of the IOM sampler free in the air at a 0-degree orientation to $1.0 \mathrm{~m} / \mathrm{s}$ freestream airflow with corresponding experimental data from (Li, 1999). See Figure 4.34.

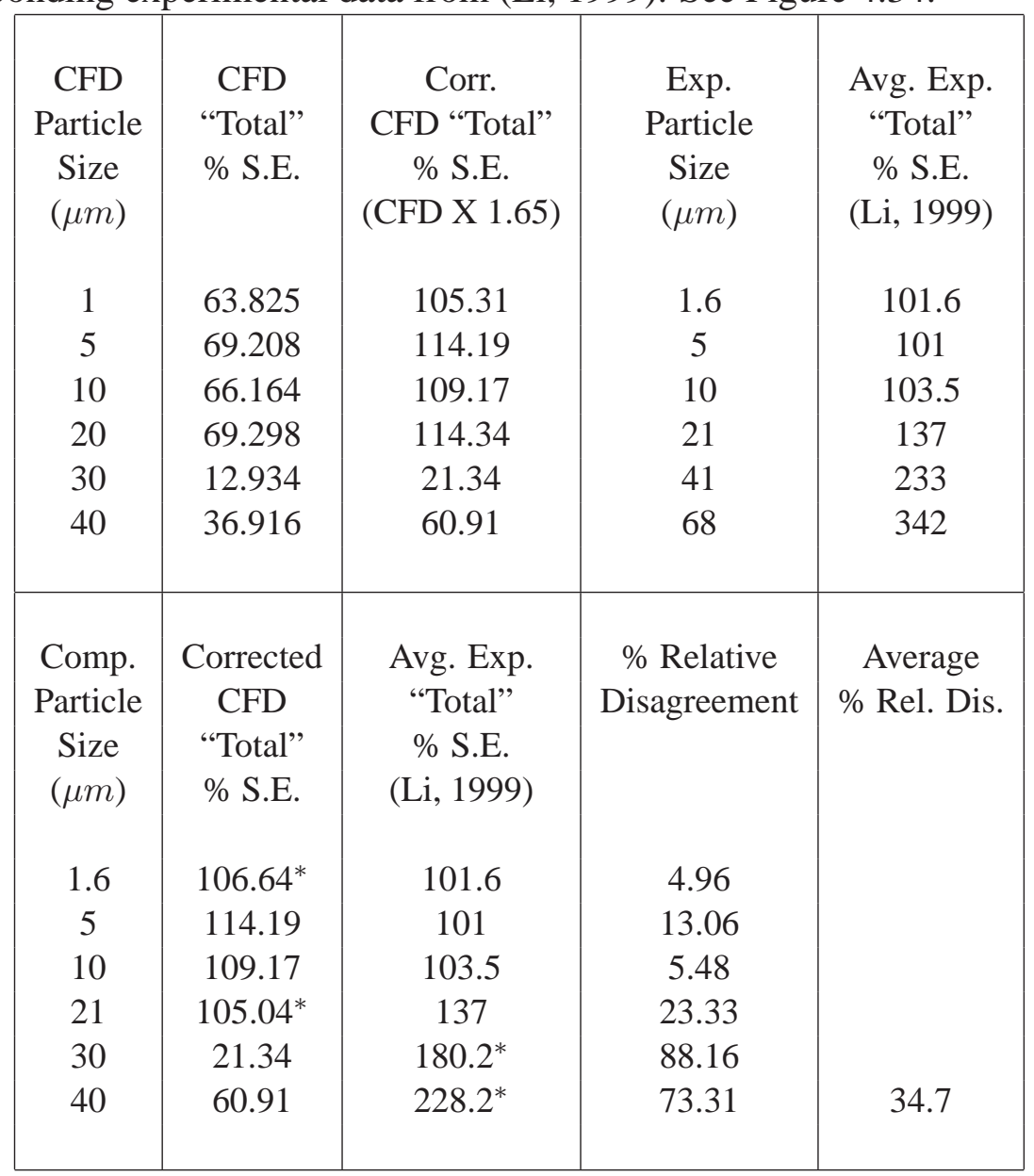

S.E. $=$ Sampling Efficiency; Corr. $=$ Corrected; Exp. $=$ Experiment; Avg. $=$ Average Comp. $=$ Comparison $;{ }^{*}=$ Interpolated;

S.E. $=$ Sampling Efficiency; Rel. $=$ Relative; Dis. $=$ Disagreement 
Table A.21: Calculated and corrected "total" sampling efficiencies from CFD simulation of the IOM sampler free in the air at a 90-degree orientation to $1.0 \mathrm{~m} / \mathrm{s}$ freestream airflow with corresponding experimental data from (Li, 1999). See Figure 4.35.

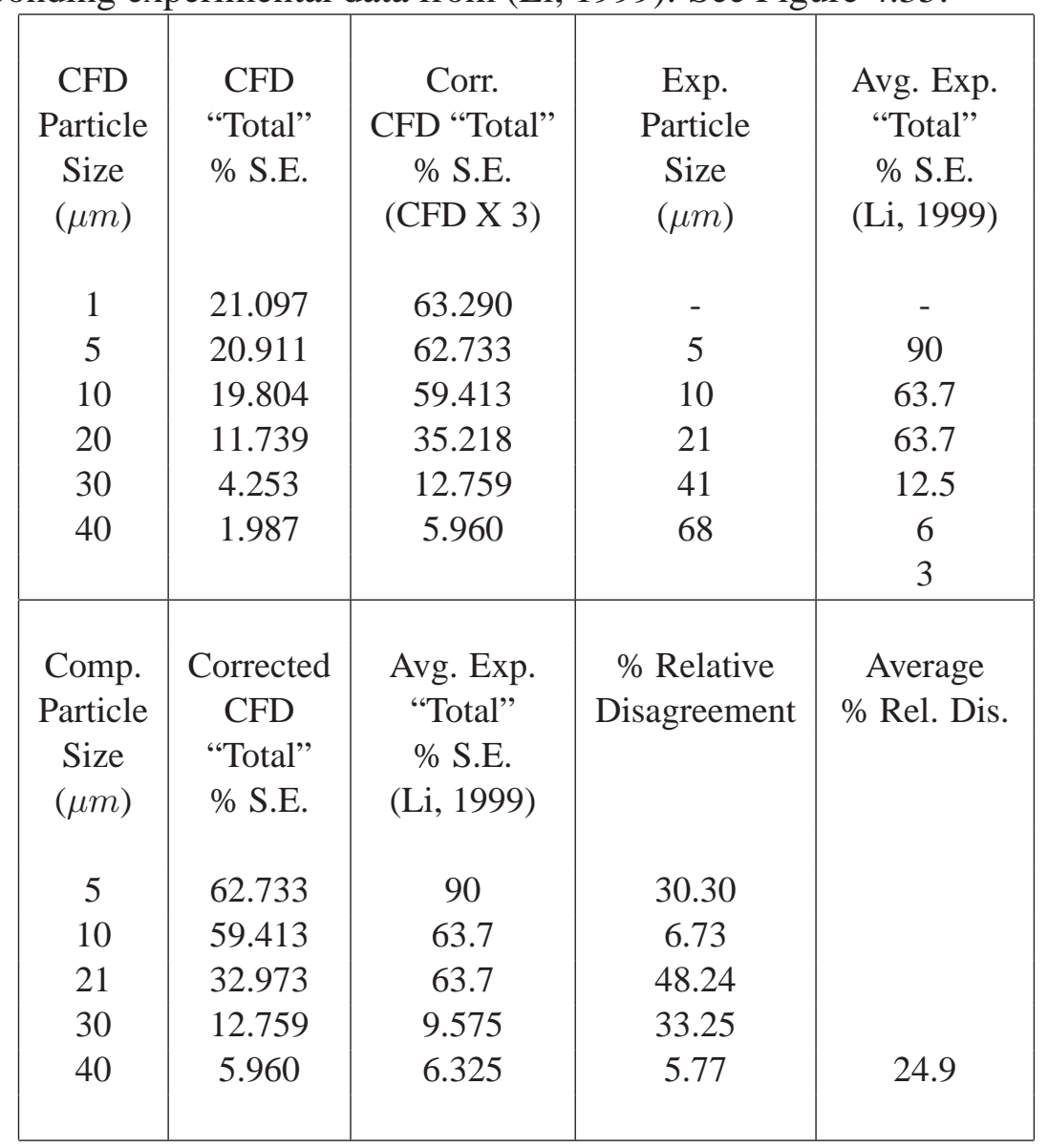

S.E. $=$ Sampling Efficiency; Corr. $=$ Corrected; Exp. $=$ Experiment; Avg. $=$ Average Comp. $=$ Comparison; ${ }^{*}=$ Interpolated;

S.E. $=$ Sampling Efficiency; Rel. $=$ Relative; Dis. $=$ Disagreement 
Table A.22: Calculated and corrected "total" sampling efficiencies from CFD simulation of the IOM sampler free in the air at a 180-degree orientation to $1.0 \mathrm{~m} / \mathrm{s}$ freestream airflow with corresponding experimental data from (Li, 1999). See Figure 4.36.

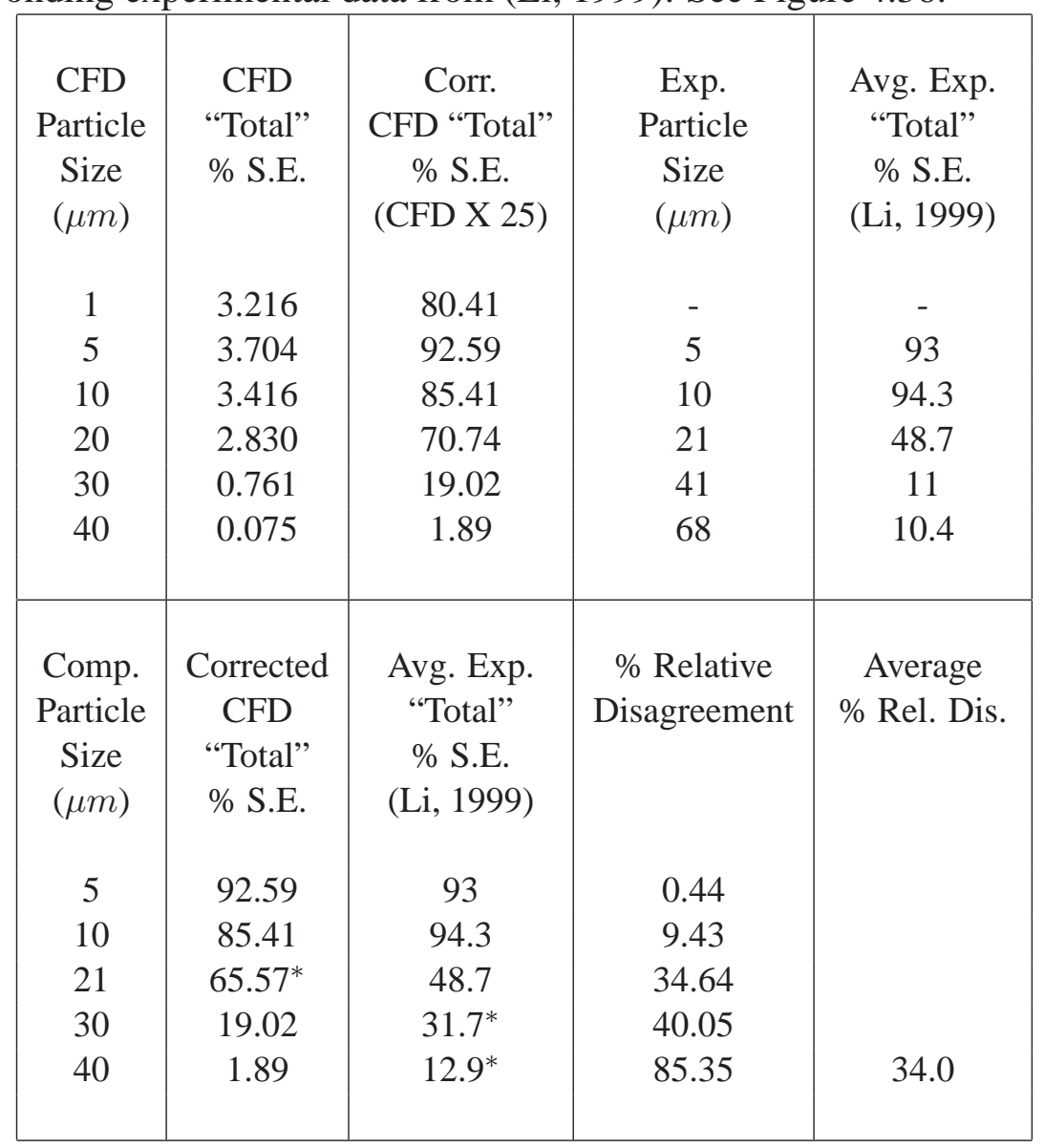

S.E. $=$ Sampling Efficiency; Corr. $=$ Corrected; Exp. $=$ Experiment; Avg. $=$ Average Comp. $=$ Comparison ${ }^{*}=$ Interpolated;

S.E. = Sampling Efficiency; Rel. = Relative; Dis. = Disagreement 
Table A.23: Calculated and corrected "Witschger" averaged sampling efficiencies from CFD simulation of the IOM sampler mounted on a box-shaped bluff body in $0.5 \mathrm{~m} / \mathrm{s}$ freestream airflow with corresponding experimental data from (Kenny et al. 1997). See Figure 4.37.

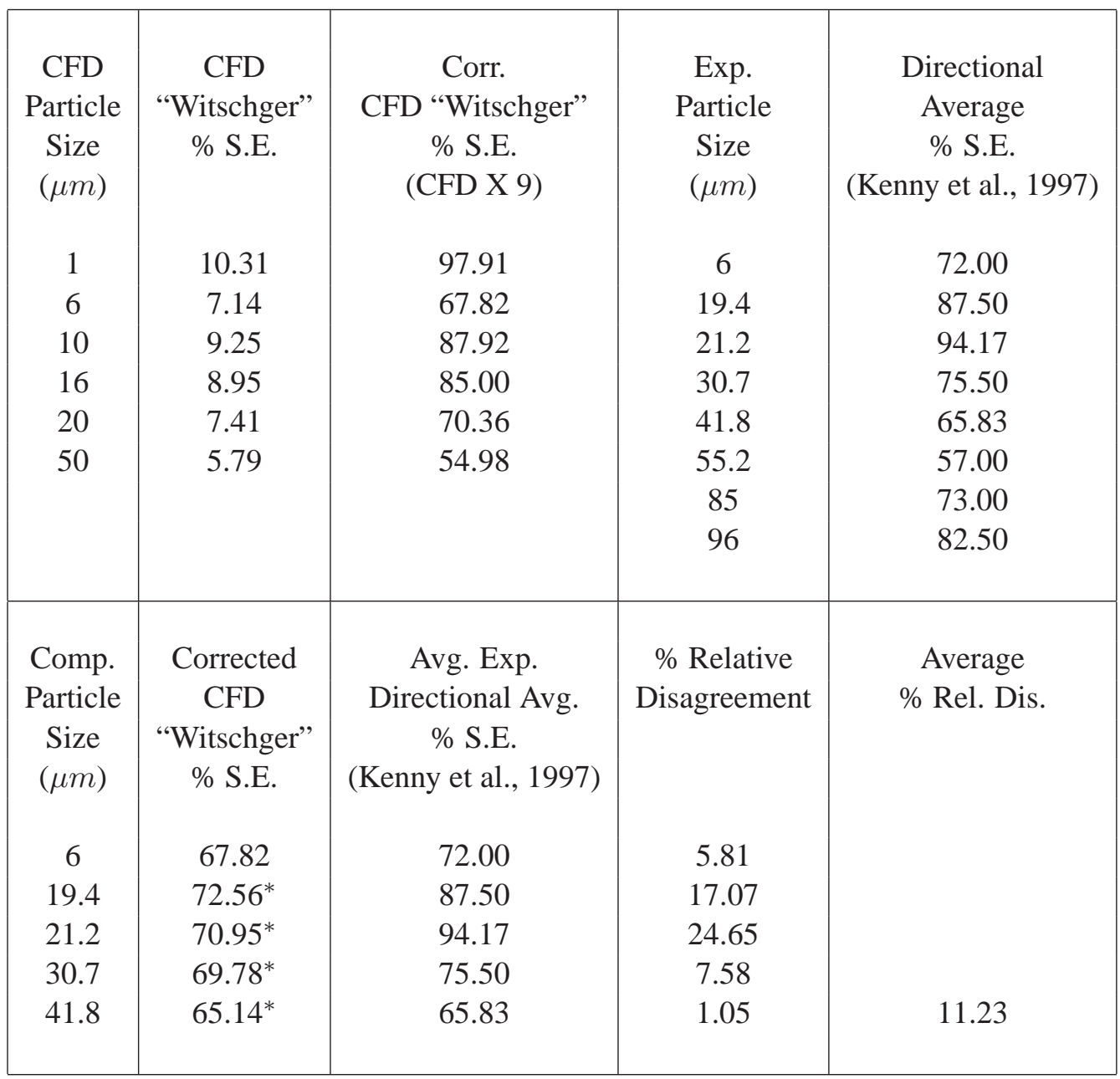

S.E. = Sampling Efficiency; Corr. = Corrected; Exp. = Experiment;

Avg. $=$ Average $;$ Comp. $=$ Comparison ${ }^{*}=$ Interpolated;

S.E. $=$ Sampling Efficiency; Rel. $=$ Relative; Dis. $=$ Disagreement

"Witschger" = Directional Averaging by Eqn. 4.1 
Table A.24: Calculated and corrected " $3 X$ " sampling efficiencies from CFD simulation of the IOM sampler mounted on a box-shaped bluff body in $0.5 \mathrm{~m} / \mathrm{s}$ freestream airflow with corresponding experimental data from (Kenny et al. 1997). See Figure 4.38.

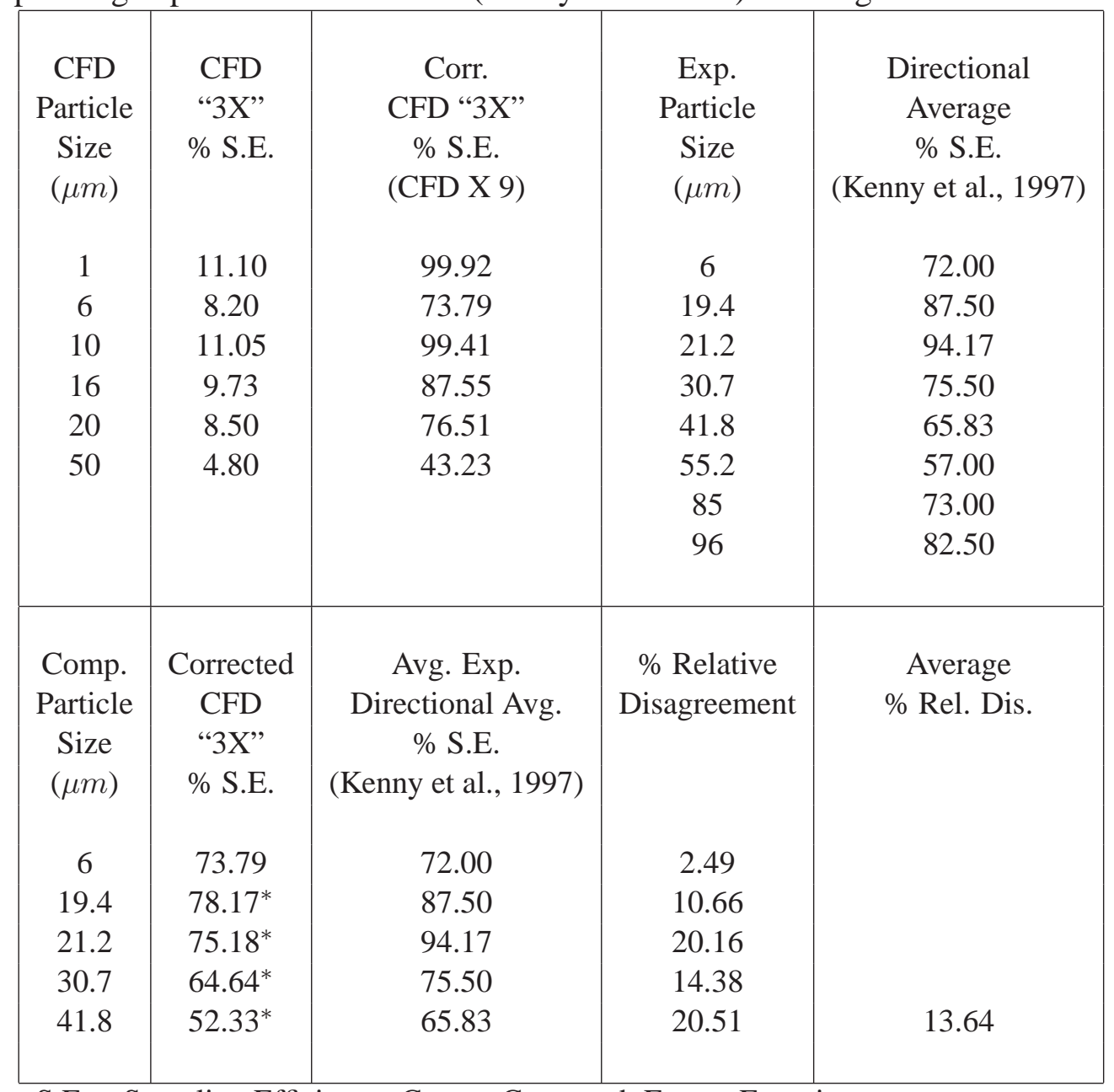

S.E. $=$ Sampling Efficiency; Corr. $=$ Corrected; Exp. $=$ Experiment;

Avg. $=$ Average Comp. $=$ Comparison $;{ }^{*}=$ Interpolated;

S.E. = Sampling Efficiency; Rel. = Relative; Dis. = Disagreement

"3X" = Directional Averaging by Eqn. 4.2 
Table A.25: Sampling efficiency values from (Kenny et al. 1997) for the IOM sampler mounted on a step-wise rotating mannequin in a wind tunnel. See Figure 4.39.

\begin{tabular}{|c|c|c|c|}
\hline $\begin{array}{c}\text { Wind } \\
\text { Speed } \\
(\mathrm{m} / \mathrm{s})\end{array}$ & $\begin{array}{c}\text { Particle } \\
\text { Size } \\
(\mu m)\end{array}$ & $\begin{array}{c}\text { Samp. } \\
\text { Effic. } \\
\%\end{array}$ & $\begin{array}{c}\text { Standard } \\
\text { Deviation }\end{array}$ \\
1 & 6 & 51.17 & 12.06 \\
1 & 19.4 & 33.83 & 6.82 \\
1 & 21.2 & 30.33 & 5.89 \\
1 & 23.3 & 25.17 & 9.70 \\
1 & 30.7 & 23.17 & 7.19 \\
1 & 41.8 & 16.33 & 7.99 \\
1 & 55.2 & 39.17 & 22.73 \\
1 & 81 & 55.17 & 12.43 \\
1 & 96 & 84.67 & 18.08 \\
& & & \\
4 & 7.5 & 71.72 & 4.94 \\
4 & 19.4 & 57.06 & 8.76 \\
4 & 21.2 & 62.28 & 10.94 \\
4 & 23.3 & 53.83 & 7.61 \\
4 & 30.7 & 47.39 & 9.57 \\
4 & 41.8 & 39.06 & 9.20 \\
4 & 55.2 & 41.11 & 9.41 \\
4 & 81 & 43.11 & 11.18 \\
4 & 96 & 41.94 & 13.78 \\
& & & \\
& & & \\
& & &
\end{tabular}


Table A.26: Calculated and corrected " $3 \mathrm{X}$ " and "Witschger" averaged sampling efficiency values from CFD simulation of the IOM sampler mounted on a box-shaped bluff body in 1.25 and $2.0 \mathrm{~m} / \mathrm{s}$ freestream airflow. See Figure 4.39 .

\begin{tabular}{|c|c|c|c|c|c|}
\hline $\begin{array}{c}\text { Wind } \\
\text { Speed } \\
(\mathrm{m} / \mathrm{s})\end{array}$ & $\begin{array}{c}\text { Particle } \\
\text { Size } \\
(\mu m)\end{array}$ & $\begin{array}{c}\text { 3X Avg. } \\
\text { Samp. Effic. } \\
(\%)\end{array}$ & $\begin{array}{c}\text { Corrected } \\
\text { 3X Avg. } \\
(\%)\end{array}$ & $\begin{array}{c}\text { Wits. Avg. } \\
\text { Samp. Effic } \\
(\%)\end{array}$ & $\begin{array}{c}\text { Corrected } \\
\text { Wits Avg. } \\
(\%)\end{array}$ \\
& & & & & \\
1.25 & 1 & 5.67 & 113.34 & 5.01 & 100.16 \\
1.25 & 6 & 2.29 & 45.84 & 2.68 & 53.61 \\
1.25 & 10 & 3.62 & 72.33 & 3.20 & 64.05 \\
1.25 & 16 & 3.78 & 75.57 & 3.08 & 61.63 \\
1.25 & 20 & 3.18 & 63.53 & 3.41 & 68.26 \\
1.25 & 50 & 4.19 & 83.89 & 3.61 & 72.26 \\
& & & & & \\
& & & Corr. (20) & \\
2.0 & 1 & 2.05 & 61.59 & 1.81 & 54.17 \\
2.0 & 6 & 3.06 & 91.76 & 2.81 & 84.44 \\
2.0 & 10 & 2.18 & 65.38 & 2.11 & 63.19 \\
2.0 & 16 & 1.55 & 46.59 & 1.68 & 50.41 \\
2.0 & 20 & 2.71 & 81.43 & 2.55 & 76.54 \\
2.0 & 50 & 6.63 & 198.80 & 5.21 & 156.31 \\
& & & & & \\
\hline
\end{tabular}

Samp. Effic. = Sampling Efficiency; Corr. $=$ Corrected

Wits. = Witschger; Avg. = Average 


\section{Appendix B}

\section{Computational Resources}

\section{B.1 Overview}

Two computers were evaluated for their performance in solving computational fluid dynamics simulations of an air sampling device used for monitoring dust levels in workplace environments. The first computer was a 32-bit SUN 6000 Enterprise Server, with eighteen 333-MHz processors, and 14 Gigabytes of RAM in a shared-memory architecture. The second computer was a 32-bit Linux Networx XEON Cluster with eight 3.06-GHz processors, and 8 Gigabytes of RAM in a distributed-memory architecture.

Computational grids of 95,000 cells, 250,000 cells, and 2,570,000 cells that approximated the geometry of the personal sampler were used as the comparative benchmarks for the performance evaluation. For the two smaller grids, comparisons were made at 1, 2, 4, and 8 processors for 1,000-iteration jobs. For the largest grid, comparisons were made at 
2, 4, and 8 processors for $601,1,000$, and 4,000 iterations. An additional comparison was made at 8 processors for 9,980 iterations.

The results of the benchmark comparison showed that the XEON linux cluster outperformed the SUN 6000 by up to $800 \%$ for large jobs run on 2 processors. However, for smaller jobs (less than 250,000 cells), the XEON cluster outperforms the SUN by no more than $300 \%$, and in some cases by much less.

\section{B.2 Background}

The National Institute for Occupational Safety and Health (NIOSH) is a Federal Agency within the Centers for Disease Control and Prevention with a primary focus on reducing and eliminating disease and injury caused by hazards within workplace environments. NIOSH realizes this goal through research studies conducted in laboratories as well as evaluations of working conditions of almost every job type found in the United States.

Since its beginnings in 1970, NIOSH has maintained state-of-the-art research tools and techniques in order to better study the health and safety of our Nation's workers. In the mid 1990's, NIOSH built a new research division for fundamental science and began to give attention to computer simulation as a way of understanding the interactions of complex systems.

An area that received attention was computational chemistry and biology. Simulations 
were made of molecular interactions, transport of materials through cells, and chemicaltissue interactions.

At about the same time, computational fluid dynamics (CFD) was also getting some attention. NIOSH funded several studies that gave greater understanding to the flow dynamics of bypass leakage in air samplers as well as airflow behavior near the inlets of air samplers. These studies demonstrated that commercially available CFD tools were suitable for research pertinent to the field of safety and health. CFD research on air sampling devices was continued and simulations were created for a number of air samplers commonly used in workplace environments.

Most of the early calculations were performed on a SUN 6000 Enterprise Server using the FLUENT CFD software package. Typically jobs were run on 4 or 8 processors, were limited to 5 million cells, and could take up to a month or more to reach convergence. In order to increase performance, upgrades were made to the SUN 6000 as funding was available, however in 2001, the architecture was maxed at eighteen 333-MHz processors and 14 Gigs of RAM. With limited funding available, the purchase of a replacement SUN 6000 server was unlikely, and thus attention was given to the linux cluster environment as a possible alternative.

In CDC there was already precedent for use of linux clusters in computational research, with one in Morgantown, WV dedicated to computational biology and another in Atlanta, GA for small-pox research. Therefore, the acquisition of a linux cluster for additional computational applications, such as CFD, was made. In 2003, a Linux Networx XEON 
Cluster with eight $3.06 \mathrm{GHz}$ processors was purchased for less than 1/10th the price of a replacement SUN 6000.

\section{B.3 Performance Evaluation Methodology}

\section{B.3.1 Simulation Benchmarks}

Comparisons between the two computers were made based on in-house simulation benchmarks of a personal aerosol sampling device. Computational grids for the simulation were made of the GSP air sampler (BIA, Sankt-Augustin, Germany; BGI, Waltham, MA, USA) at three different grid sizes: 95,000 cells, 250,000 cells, and 2,570,000 cells. In CFD, it is standard practice to conduct simulations at a range of different grid sizes in order to see if there is an effect of the grid on the values resulting from the simulation. For our applications in parallel performance evaluation, it is useful to see differences in the communication and memory requirements. Therefore, three different grid sizes were used in this evaluation, which is the minimum number that should be used.

The simulations were run in FLUENT 6.0 (Fluent, Inc., Lebanon, NH), a commercially available CFD software package. The software is based on numerical discretizations of the Navier-Stokes equations, which are the governing equations of fluid flow. Solutions are achieved by solving algebraic representations of the discretizations in a finite-volume iterative manner. A job is considered finished when all of the calculation results no longer 
change from iteration to iteration, i.e. when the residuals (current value minus previous value) become constant, and ideally zero.

To start the simulations, boundary conditions were applied in order to recreate the state found in wind-tunnel testing environments that have been used to evaluate the performance of the sampler. In this way, the results from the simulations could be validated with results from wind-tunnel experiments and the accuracy of the simulation could be determined. Boundary conditions applied in the benchmarks can be seen in Table B.1.

Finally, the number of iterations the simulations were run was varied in order to see if there was a difference in the rate of calculation for shorter and longer iterations. Processors are often evaluated with extremely short running jobs so the effect of communication to the nodes may not be an important factor in those kinds of evaluations. It is common knowledge, however, that communication in clusters is a major bottleneck. Furthermore, by running the jobs similarly to how they would be run for research applications, the slowdowns caused by system overloading, i.e. more jobs running than processors available, can also be seen.

A total of 43 simulations were run for a sum of 2,530,041.4 seconds. On the SUN 6000, 17 jobs were run, and on the XEON linux cluster, 26 jobs were run. 
Table B.1: Boundary conditions, grid density, and iterations for simulation benchmarks used in this performance evaluation.

\begin{tabular}{|c|c|c|c|c|c|c|c|}
\hline computer & $\begin{array}{l}\text { aerosol } \\
\text { sampler }\end{array}$ & $\begin{array}{c}\text { freestream } \\
\text { wind speed } \\
\text { (turb. intensity) }\end{array}$ & $\begin{array}{c}\text { sampler } \\
\text { inlet velocity } \\
\text { (turb. intensity) }\end{array}$ & $\begin{array}{c}\text { turbulence } \\
\text { model }\end{array}$ & $\begin{array}{c}\text { grid } \\
\text { density }\end{array}$ & iterations & processors \\
\hline linux & GSP & $0.5 \mathrm{~m} / \mathrm{s}$ & $1.5 \mathrm{~m} / \mathrm{s}$ & k-e & 0.0005 & 601 & $2,4,8$ \\
\hline linux & GSP & $0.5 \mathrm{~m} / \mathrm{s}$ & $1.5 \mathrm{~m} / \mathrm{s}$ & k-e & 0.0005 & 1000 & $2,4,8$ \\
\hline linux & GSP & $0.5 \mathrm{~m} / \mathrm{s}$ & $1.5 \mathrm{~m} / \mathrm{s}$ & k-e & 0.0005 & 4000 & 8 \\
\hline linux & GSP & $0.5 \mathrm{~m} / \mathrm{s}$ & $1.5 \mathrm{~m} / \mathrm{s}$ & k-e & 0.0005 & 9980 & 8 \\
\hline linux & GSP & $0.5 \mathrm{~m} / \mathrm{s}$ & $1.5 \mathrm{~m} / \mathrm{s}$ & k-e & 0.0005 & 10980 & 8 \\
\hline linux & GSP & $0.5 \mathrm{~m} / \mathrm{s}$ & $1.5 \mathrm{~m} / \mathrm{s}$ & k-e & 0.0005 & 11980 & 8 \\
\hline linux & GSP & $0.5 \mathrm{~m} / \mathrm{s}$ & $1.5 \mathrm{~m} / \mathrm{s}$ & k-e & 0.001 & 1000 & $1,2,4,8$ \\
\hline linux & GSP & $0.5 \mathrm{~m} / \mathrm{s}$ & $1.5 \mathrm{~m} / \mathrm{s}$ & k-e & 0.0015 & 1000 & $1,2,4,8$ \\
\hline sun & GSP & $0.5 \mathrm{~m} / \mathrm{s}$ & $1.5 \mathrm{~m} / \mathrm{s}$ & k-e & 0.0005 & 601 & $2,4,8$ \\
\hline sun & GSP & $0.5 \mathrm{~m} / \mathrm{s}$ & $1.5 \mathrm{~m} / \mathrm{s}$ & k-e & 0.0005 & 1000 & $2,4,8$ \\
\hline sun & GSP & $0.5 \mathrm{~m} / \mathrm{s}$ & $1.5 \mathrm{~m} / \mathrm{s}$ & k-e & 0.0005 & 4000 & 4,8 \\
\hline sun & GSP & $0.5 \mathrm{~m} / \mathrm{s}$ & $1.5 \mathrm{~m} / \mathrm{s}$ & k-e & 0.0005 & 9980 & 8 \\
\hline sun & GSP & $0.5 \mathrm{~m} / \mathrm{s}$ & $1.5 \mathrm{~m} / \mathrm{s}$ & k-e & 0.001 & 1000 & $1,2,4,8$ \\
\hline sun & GSP & $0.5 \mathrm{~m} / \mathrm{s}$ & $1.5 \mathrm{~m} / \mathrm{s}$ & k-e & 0.0015 & 1000 & $1,2,4,8$ \\
\hline
\end{tabular}




\section{B.3.2 Elapsed Time and Ratings}

Elapsed time (e.t.), also called wall-clock time, was recorded for each benchmark and the rating $(r)$ calculated as follows:

$$
r=86,400 /(\text { e.t. })
$$

where 86,400 is the number of seconds in day, and $r$ is a measure of the number of benchmarks that can be run in a day.

\section{B.4 Results}

\section{B.4.1 Rating}

Benchmarks were made for the coarse and medium grid sizes for 1,000 iteration calculations on 1, 2, 4, and 8 processors; at the fine grid size for 601 and 1,000 iterations on 2, 4, and 8 processors; at the fine grid size for 4,000 iterations on 4 and 8 processors; and at $9,980,10,980$, and 11,980 iterations on 8 processors for the linux cluster only. The results are presented in Tables B.2, B.3, and B.4, and plotted in Figures B.1, B.2, B.3, and B.4. The plots also show the increase in performance when the calculation is run on the SUN 6000 versus when it is run on the linux cluster. 
For the case of 1,000 iterations run on the coarse grid size (see Figure B.1), the generally low increase (especially at 4 processors) is due to the small size of the job and the impact of secondary activities such as GUI control, networking, etc., on the PC architecture's distributed memory and low BUS speed, which would otherwise be needed for communication between nodes.

For 1,000 iterations run on the medium grid size (Figure B.2), the linux cluster outperforms the SUN by about 3 times at 1 and 2 processors and by nearly 2 times at 4 processors. However, at 8 processors, there is a smaller performance increase.

Significant performance increase occurs for 1,000 iteration jobs run at the finest grid size (Figure B.3), where the linux cluster outperforms the SUN by nearly 8 times for 2 processors, 6.5 times for 4 processors, and a respectable 5 times for 8 processors. It is interesting to note that at 2 processors, the linux cluster nearly reaches its maximum potential increase over the SUN when considering processor speed increase: $3.06 \mathrm{GHz}$ versus $333 \mathrm{MHz}$, or 9.2 times faster.

Finally, for iteration comparison, simulations having the finest gride size run at 601 iterations show performance increase similar to the increase at 1000 iterations, although when processor scaling has reached 8 processors, the increase is only 3.9 times (see Figure B.4). 


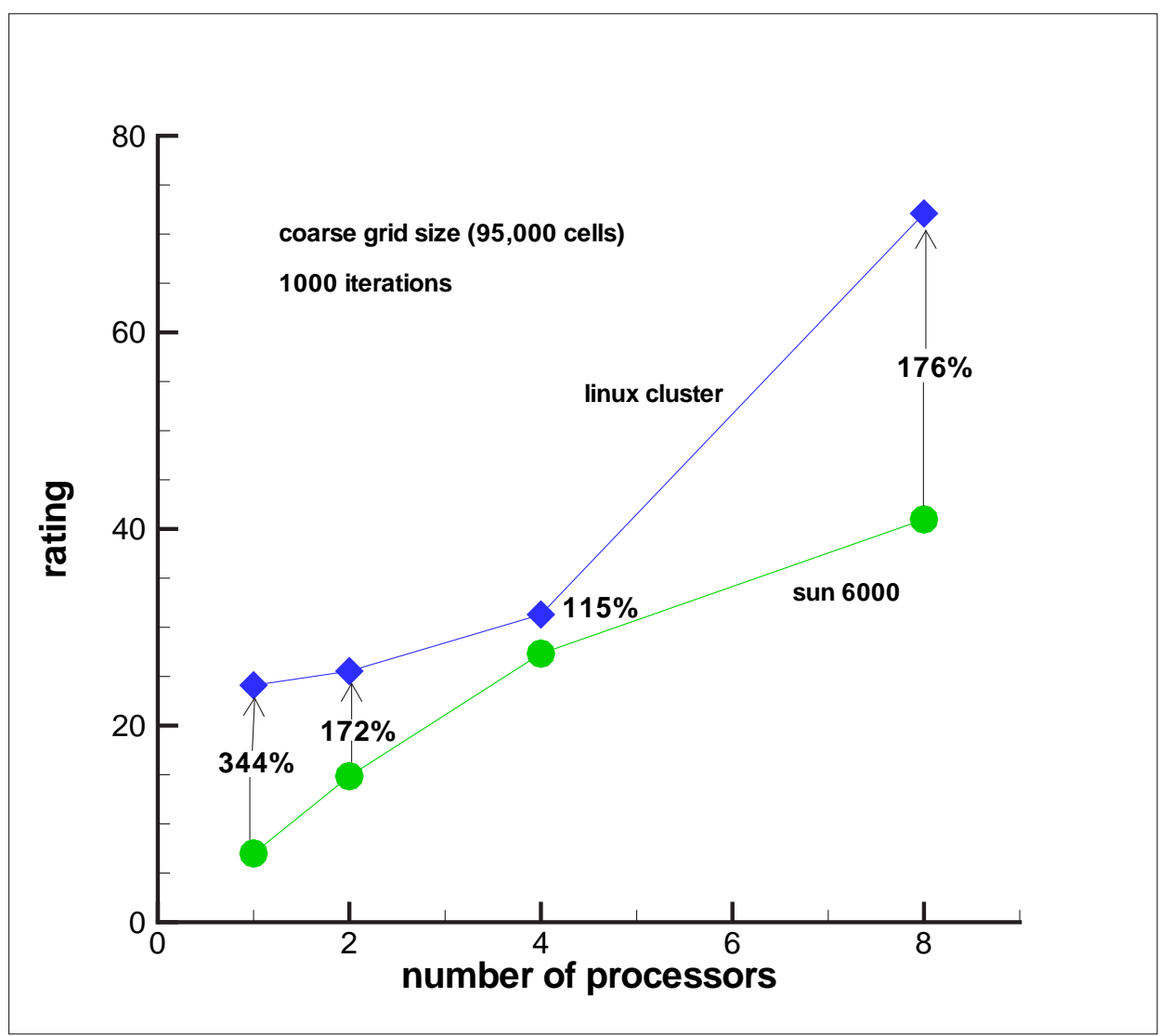

Figure B.1: Comparison of parallel scaling between SUN 6000 and Linux Networx XEON Cluster for calculations run for 1000 iterations on coarse grid size. 
Table B.2: Results of simulation benchmarks for the SUN 6000 and linux cluster calculations of CFD models at medium (0.001) and coarse (0.0015) grid sizes.

\begin{tabular}{|c|c|c|c|c|c|}
\hline computer & $\begin{array}{c}\text { grid } \\
\text { density }\end{array}$ & iterations & processors & $\begin{array}{l}\text { elapsed time } \\
\text { (seconds) }\end{array}$ & rating \\
\hline sun & medium & 1000 & 1 & 31492.4 & 2.74 \\
\hline sun & medium & 1000 & 2 & 17737.7 & 4.87 \\
\hline sun & medium & 1000 & 4 & 7608.9 & 11.36 \\
\hline sun & medium & 1000 & 8 & 4098.6 & 21.08 \\
\hline sun & coarse & 1000 & 1 & 12353.8 & 6.99 \\
\hline sun & coarse & 1000 & 2 & 5817.4 & 14.85 \\
\hline sun & coarse & 1000 & 4 & 3162.9 & 27.32 \\
\hline sun & coarse & 1000 & 8 & 2108.6 & 40.98 \\
\hline linux & medium & 1000 & 1 & 9117.2 & 9.48 \\
\hline linux & medium & 1000 & 2 & 5725.1 & 15.09 \\
\hline linux & medium & 1000 & 4 & 4043.5 & 21.37 \\
\hline linux & medium & 1000 & 8 & 2888.6 & 29.91 \\
\hline linux & coarse & 1000 & 1 & 3592.6 & 24.05 \\
\hline linux & coarse & 1000 & 2 & 3382.7 & 25.54 \\
\hline linux & coarse & 1000 & 4 & 2759.2 & 31.31 \\
\hline linux & coarse & 1000 & 8 & 1198.5 & 72.09 \\
\hline
\end{tabular}




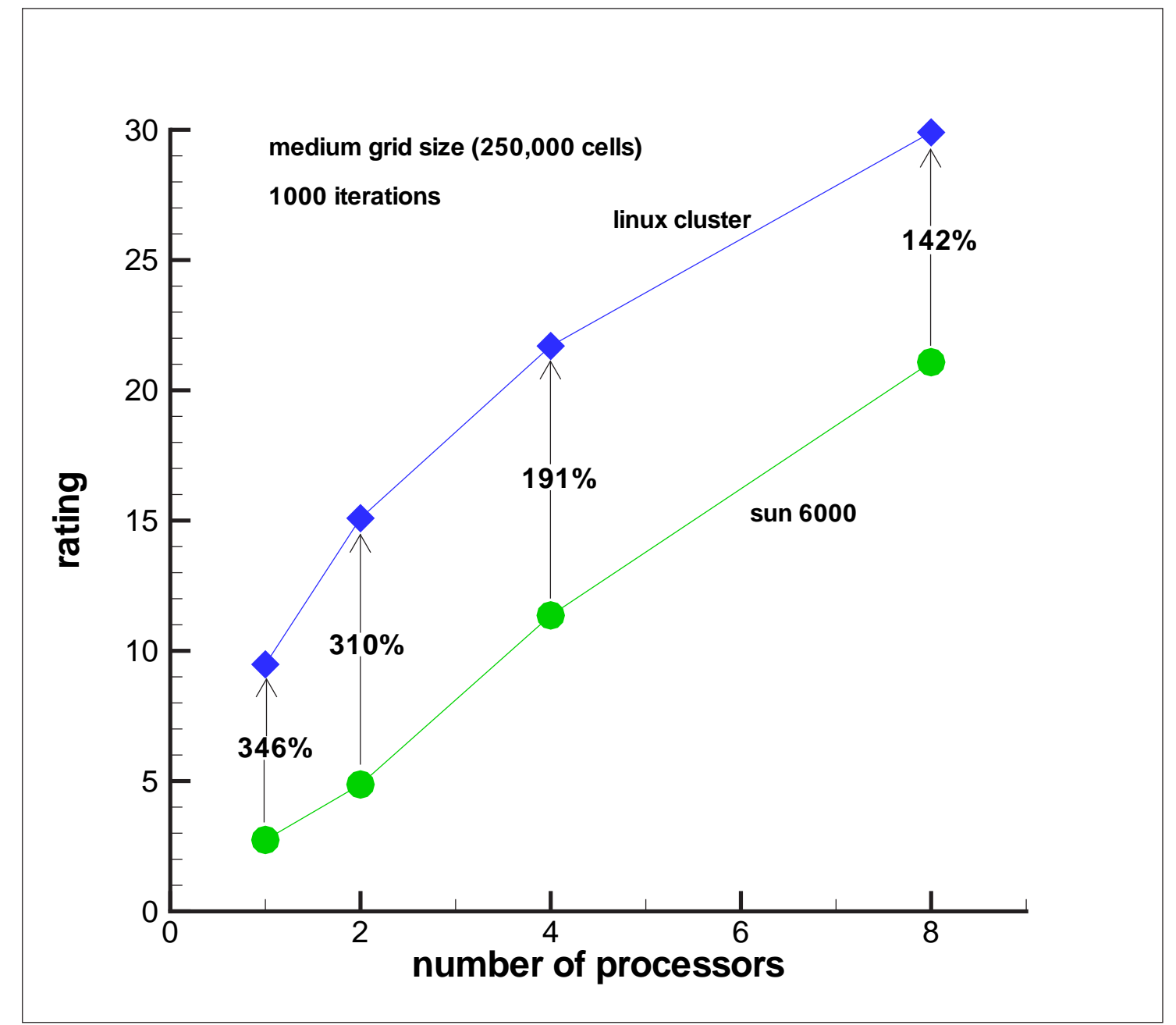

Figure B.2: Comparison of parallel scaling between SUN 6000 and Linux Networx XEON Cluster for calculations run for 1000 iterations on medium grid size. 


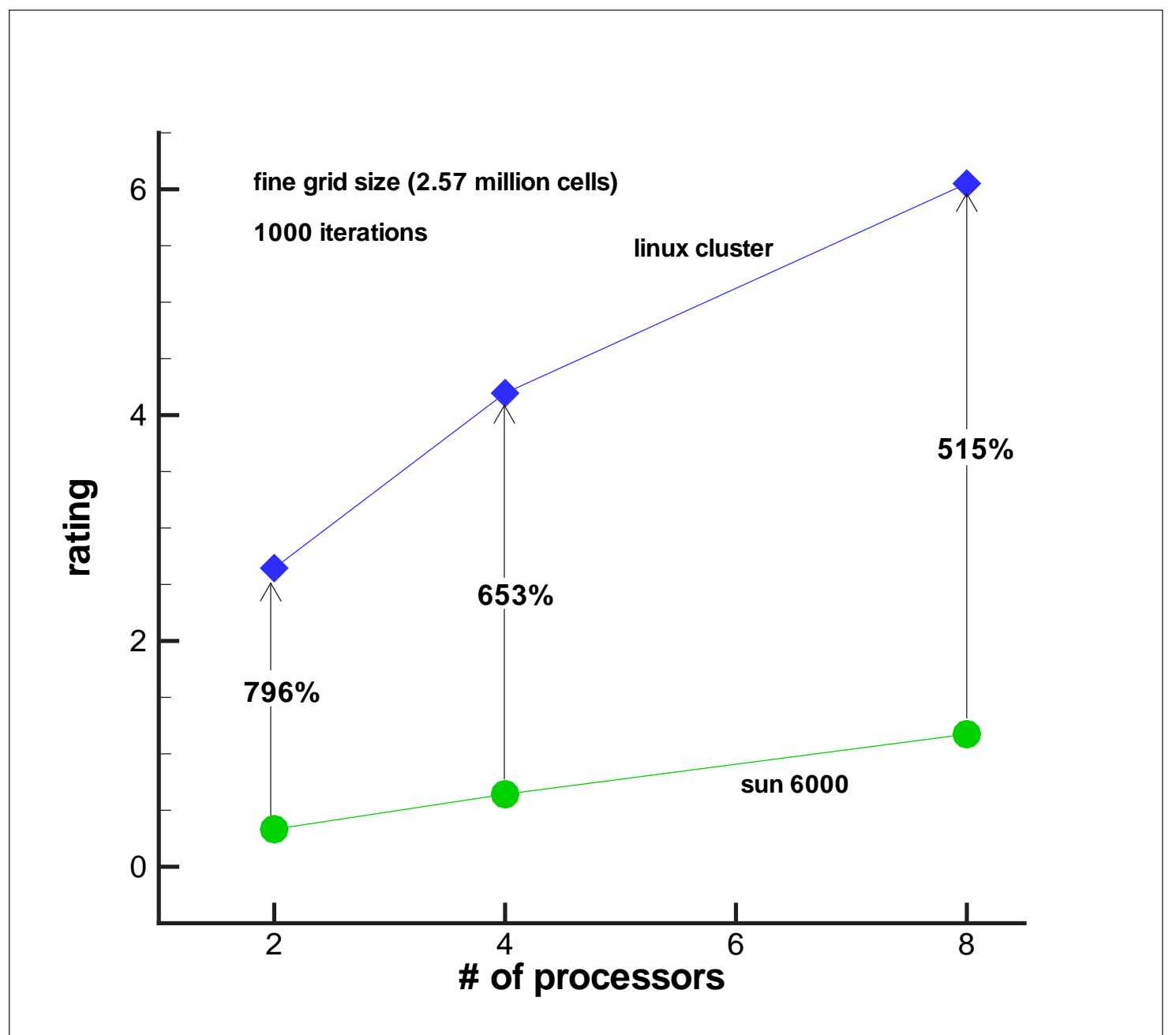

Figure B.3: Comparison of parallel scaling between SUN 6000 and Linux Networx XEON Cluster for calculations run for 1000 iterations on fine grid size. 
Table B.3: Results of simulation benchmarks for SUN 6000 calculations of CFD models at the fine grid size $(0.0005)$.

\begin{tabular}{|c|c|c|c|c|c|}
\hline computer & $\begin{array}{c}\text { grid } \\
\text { density }\end{array}$ & iterations & processors & $\begin{array}{c}\text { elapsed time } \\
\text { (seconds) }\end{array}$ & rating \\
\hline sun & fine & 601 & 2 & 153175.5 & 0.56 \\
\hline sun & fine & 601 & 4 & 65005.1 & 1.33 \\
\hline sun & fine & 601 & 8 & 33111.6 & 2.61 \\
\hline sun & fine & 1000 & 2 & 260021.8 & 0.33 \\
\hline sun & fine & 1000 & 4 & 134576.6 & 0.64 \\
\hline sun & fine & 1000 & 8 & 73609.9 & 1.17 \\
\hline sun & fine & 4000 & 4 & 329215.8 & 0.26 \\
\hline sun & fine & 4000 & 8 & 230769.2 & 0.37 \\
\hline sun & fine & 9980 & 8 & 491016.8 & 0.18 \\
\hline
\end{tabular}


Table B.4: Results of simulation benchmarks for linux cluster calculations of CFD models at the fine grid size $(0.0005)$.

\begin{tabular}{|c|c|c|c|c|c|}
\hline computer & $\begin{array}{c}\text { grid } \\
\text { density }\end{array}$ & iterations & processors & $\begin{array}{c}\text { elapsed time } \\
\text { (seconds) }\end{array}$ & rating \\
\hline linux & fine & 601 & 2 & 19649.2 & 4.40 \\
\hline linux & fine & 601 & 4 & 12362.8 & 6.99 \\
\hline linux & fine & 601 & 8 & 8565.8 & 10.09 \\
\hline linux & fine & 1000 & 2 & 32669.5 & 2.64 \\
\hline linux & fine & 1000 & 4 & 20595.3 & 4.20 \\
\hline linux & fine & 1000 & 8 & 14279.2 & 6.05 \\
\hline linux & fine & 4000 & 8 & 56775.0 & 1.52 \\
\hline linux & fine & 9980 & 8 & 140156.6 & 0.62 \\
\hline linux & fine & 10980 & 8 & 154108.0 & 0.56 \\
\hline fine & 11980 & 8 & 168058.8 & 0.51 \\
\hline
\end{tabular}




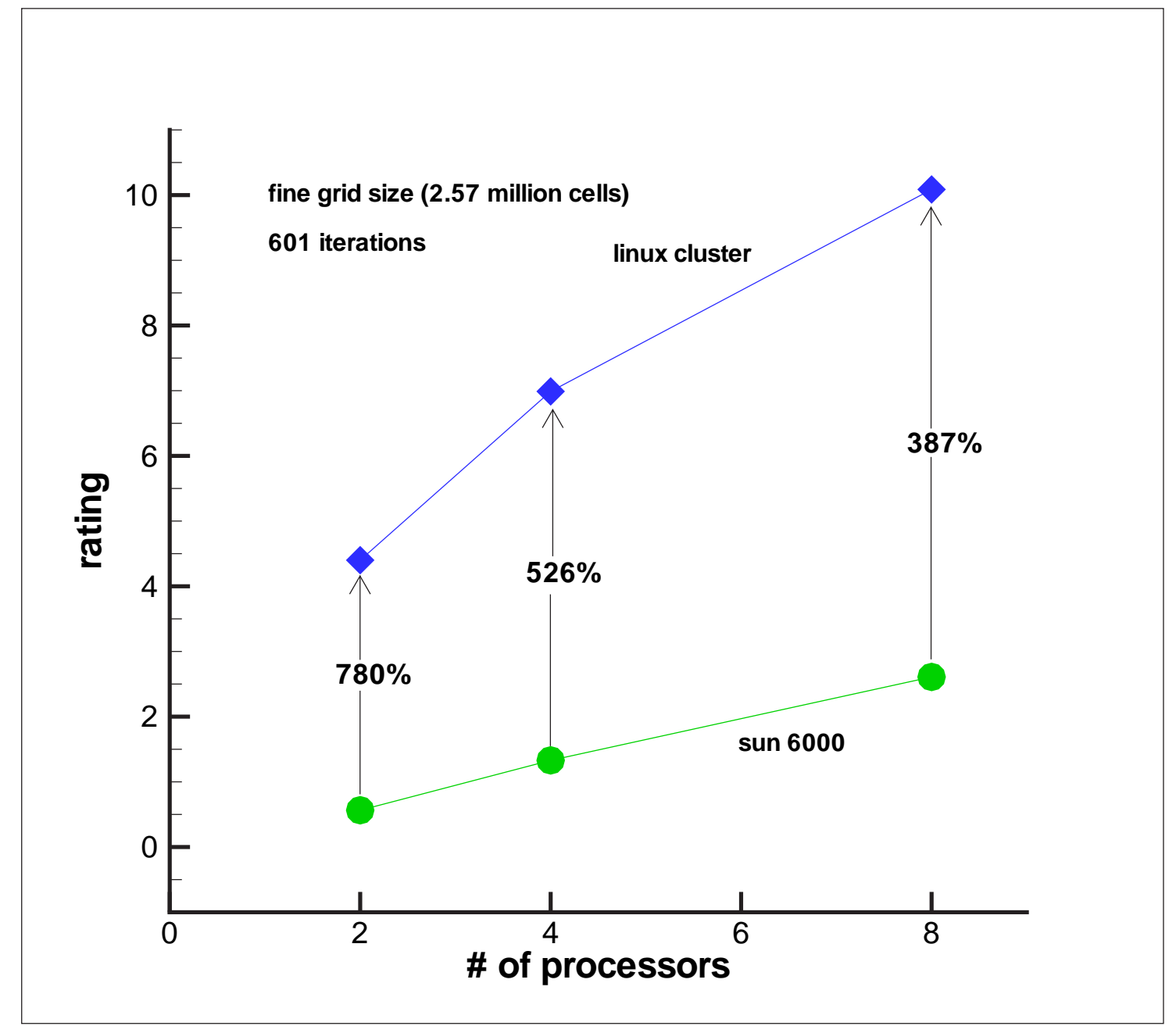

Figure B.4: Comparison of parallel scaling between SUN 6000 and Linux Networx XEON Cluster for calculations run for 601 iterations on fine grid size. 


\section{B.4.2 Parallel Scaling}

To determine the ideal number of processors to use for a calculation, the speedup and efficiency were determined. Tables B.5 and B.6 show the efficiency of increasing the number of processors for a particular calculation. Efficiency is determined from: ((relative speedup)/(\# times increased)) X 100. These efficiency values are based on results from benchmarks run for 1000 iterations. The linux cluster scales best when going from 2 to 4 processors for fine grids and from 2 to 8 for coarse grids. The SUN 6000 scales very well for all processor increases.

\section{B.5 Conclusions}

Based on speed of the computers tested in this comparison, use of the linux cluster in favor of the SUN 6000 will result in decreased elapsed times when conducting CFD simulations. The decrease in time, i.e. becoming "less expensive," has the greatest benefit for large jobs that are run on four processors. 
Table B.5: Results of efficiency determinations for the XEON linux cluster.

\begin{tabular}{|c|c|c|c|c|}
\hline $\begin{array}{c}\text { processor } \\
\text { increase }\end{array}$ & $\begin{array}{c}\text { \# times } \\
\text { increased }\end{array}$ & $\begin{array}{c}\text { fine grid efficiency } \\
\text { (2.57M cells) }\end{array}$ & $\begin{array}{c}\text { medium grid efficiency } \\
\text { (250K cells) }\end{array}$ & $\begin{array}{c}\text { coarse grid efficiency } \\
\text { (95K cells) }\end{array}$ \\
\hline 1 to 2 & 2 & - & 79.65 & 53 \\
\hline 1 to 4 & 4 & - & 56.37 & 32.55 \\
\hline 1 to 8 & 8 & - & 39.45 & 37.47 \\
\hline 2 to 4 & 2 & 79.5 & 70.79 & 61.3 \\
\hline 2 to 8 & 4 & 57.25 & 49.55 & 70.56 \\
\hline 4 to 8 & 2 & 72.12 & 69.99 & 101.51 \\
\hline
\end{tabular}


Table B.6: Results of efficiency determinations for the SUN 6000.

\begin{tabular}{|c|c|c|c|c|}
\hline $\begin{array}{c}\text { processor } \\
\text { increase }\end{array}$ & $\begin{array}{c}\text { \# times } \\
\text { increased }\end{array}$ & $\begin{array}{c}\text { fine grid } \\
(2.57 \mathrm{M} \text { cells })\end{array}$ & $\begin{array}{c}\text { medium grid } \\
\text { (250K cells) }\end{array}$ & $\begin{array}{c}\text { coarse grid } \\
\text { (95K cells) }\end{array}$ \\
\hline 1 to 2 & 2 & - & 88.77 & 106.00 \\
\hline 1 to 4 & 4 & - & 103.47 & 97.65 \\
\hline 1 to 8 & 8 & - & 96.05 & 73.23 \\
\hline 2 to 4 & 2 & 96.61 & 116.56 & 91.97 \\
\hline 2 to 8 & 4 & 88.31 & 108.19 & 68.97 \\
\hline 4 to 8 & 2 & 91.41 & 92.82 & 75.00 \\
\hline
\end{tabular}




\title{
Curriculum Vitae
}

\author{
Aaron J. Bird \\ P.O. Box 368 \\ Morgantown, WV 26507 \\ 304-291-4745
}

Education

Ph.D. in Industrial and Management Systems Engineering, December 2004, West Virginia University, Morgantown, WV, USA. Dissertation: "Use of Commercial CFD Software for Assessing the Performance of Personal Aerosol Samplers,” Committee Chair: Dr. Warren R. Myers.

M.S. in Occupational Hygiene and Safety, May 1998, West Virginia University, Morgantown, WV, USA. Problem Report: "Differences in Failure Modes and Effects Analyses,” Committee Chair: Terrence C. Stobbe.

B.S. in Mathematics, May 1996, Fairmont State College, Fairmont, WV, USA. Advisor: M. Jeanne Harris.

\section{Employment}

\section{Research Industrial Hygienist (Gov. Series 9, Step 5)}

- National Institute for Occupational Safety and Health, Centers for Disease Control and Prevention. May 1998 - Present (December 2004). Work experience: Use of wind tunnel experiments and computational simulation for evaluation of aerosol samplers and airflow patterns around human bodies; Validation of computational simulations with laser-Doppler velocimetry and particle-imaging velocimetry data; Workplace exposure monitoring with existing and prototype aerosol samplers; Development of methods for indoor (non-GPS) position-tracking systems; Project proposal development and writing, with focus in design of experiments; Project and expenditures management; Development of communication/feedback cycles with stakeholders.

\section{Graduate Research Assistant}

- West Virginia University, College of Engineering and Mineral Resources, Department of Industrial and Management Systems Engineering. August 1996 - May 1998. Work experience: Hardware and software support for hazard identification project; Failure modes and effects analyses of nylon-recycling industrial process. 


\section{Lab Research Assistant}

- West Virginia University Research Corporation. May 1997 - August 1997. Work experience: Analysis of chloro-fluoro carbon in exhaled breath using gas-chromatography, mass spectrometry; Performance fit-testing evaluations of prototype respirators; Writing and implementation of visual-basic-based statistical software program for analysis of respirator performance.

\section{Lab Assistant}

- West Virginia University, College of Agriculture Forestry and Consumer Sciences, Department of Entomology. May 1991 - 1996. Work experience: Collection of insects, arthropods, arachnids, etc. from field locations for large U.S. Dept. of Agriculture study; Sorting of collected specimens by genus.

\section{Lab Assistant}

- West Virginia University, College of Arts and Sciences, Department of Chemistry. August 1991 - May 1992. Work experience: Lab preparation including titration of solutions for quality assurance, massing of chemicals for dispensing to students.

\section{Teaching/Teaching Assistant Experience}

Analysis of Engineering Data, Department of Industrial and Management Systems Engineering, West Virginia University, Fall 2001. Work experience: Instructor, Full-semester Linear Regression course for ThirdYear Industrial Engineering Students.

Industrial Hygiene Sampling and Analysis, Department of Industrial and Management Systems Engineering, West Virginia University, Spring 1998. Work experience: Assisted Instructor with Course Preparation and Grading of Assignments.

Engineering Statistics, Department of Industrial and Management Systems Engineering, West Virginia University, Spring 1998. Work experience: Assisted Instructor with Course Preparation and Grading of Assignments.

\section{Publications/Presentations}

Harper, Martin; Hallmark, Timothy; Andrew, Mike; Bird, Aaron. (2004) A Comparison of X-Ray Fluorescence and Wet Chemical Analysis of Air Filter Samples from a Scrap Lead Smelting Operation. Journal of Environmental Monitoring. Vol. 6, pp. 819-826.

Li, Jun; Celik, Ismail; Yavuz, Ibrahim; Guffey, Steven E.; Bird, Aaron J. (2003) The Effect of Turbulence and Scalar Transport Models on Prediction of Worker Exposure to Aerosols. Proceedings of FEDSM'03 4th ASME-JSME Joint Fluids Engineering Conference, Honolulu, Hawaii, USA, July 6-11, 2003. 
Smith, Jerome P. and Bird, Aaron J. (2002) Relationship of Sampling Efficiency for Manikin-Mounted Personal Samplers to Efficiency Measurements Made Independent of Manikin. Journal of Aerosol Science. Vol. 33, pp. 1235-1259.

Bird, Aaron J. (2003). Comparison of Computational Simulations of Airflow Patterns near the Inlets of the IOM Inhalable and GSP Samplers. Presented at the American Industrial Hygiene Association Conference, May 12-15, 2003, Dallas, TX.

Bird, Aaron J. (2003) Assessment of Grid Independence and Choice of Turbulence Model for CFD Simulations of Two Personal Air Sampling Devices. Presented at the Fluent User's Group Meeting, May 5-7, 2003, Manchester, NH.

Bird, Aaron J. (2002) Boundary Conditions from Wind Tunnel Measurements Used in Computational Fluid Dynamics Simulations of Torso-Mounted Air Samplers. Presented at the Interdivisional Meeting of the National Institute for Occupational Safety and Health, November 13, 2002, Mineral Wells, WV.

Bird, Aaron J. (2002) Validation of Computational Fluid Dynamics Simulations. Presented at the Interdivisional Meeting of the National Institute for Occupational Safety and Health, January 24, 2002, Mineral Wells, WV.

Bird, Aaron J. (2000) Analysis of Data from Laser-Doppler Velocimetry Measurements. Presented as part of IMSE Seminar Series, Department of Industrial and Management Systems Engineering, West Virginia University, November, 17, 2000, Morgantown, WV.

\section{Pertinent Skills and Abilities}

Software: FLUENT, GAMBIT, Paraform, Tecplot, SAS, Ensight, Design Expert, Various Microsoft Products, Adobe Illustrator.

Research: Statistical Analysis, Software Code Writing, Experiment Design, Project Development/Proposal Writing, Project Data Collection/Recording/Dissemination, Report/Manuscript Writing.

Management-Related: Communication with Stakeholders/Vendors/Collaborators, Project Organization \& Goal Achievement, Team Motivation, Budget/Expenditures Management. 
Aaron Bird, Pg. 4 of 4, December, 2004

\section{Additional Skills and Experience}

Wilderness Rescue: Trained for Confined Space and Vertical Rescues Cave Rescue Instructor - 1990 to 2000 Participated in 12 Cave Rescues in Team Leader, Vertical Rigging, and Management Roles.

Career-Related: Aerosol Physics, CFD, Experiment Design, Occupational Safety and Health, Trend Analysis, Uncertainty Analysis, Validation.

Personal: Spending time with family, Biking, Caving, Gardening, Hiking, Reading, Rock Climbing, Skiing, Sporting Events. 